\title{
TV Energy Consumption Trends and Energy-Efficiency Improvement Options
}

\author{
Won Young Park, Amol Phadke, Nihar Shah, Virginie \\ Letschert
}

Environmental Energy Technologies Division International Energy Studies Group

July 1, 2011

This work was funded by the Bureau of Oceans and International Environmental and Scientific Affairs, U.S. Department of State, and administered by the U.S. Department of Energy in support of the Super-efficient Equipment and Appliance Deployment (SEAD) initiative through the U.S. Department of Energy under Contract No. DE-AC02-05CH11231 


\section{Disclaimer}

This document was prepared as an account of work sponsored by the United States Government in support of the Super-efficient Equipment and Appliance Deployment (SEAD) initiative. While this document is believed to contain correct information, neither the United States Government nor any agencies thereof, SEAD participating Governments nor any agencies thereof, the SEAD Operating Agent, The Regents of the University of California, nor any of their employees, makes any warranty, express or implied, or assumes any legal responsibility for the accuracy, completeness, or usefulness of any information, apparatus, product, or process disclosed, or represents that its use would not infringe privately owned rights. Reference herein to any specific commercial product, process, or service by its trade name, trademark, manufacturer, or otherwise, does not necessarily constitute or imply its endorsement, recommendation, or favoring by the United States Government or any agency thereof, SEAD participating Governments or any agencies thereof, the SEAD Operating Agent, or The Regents of the University of California. The views and opinions of authors expressed herein do not necessarily state or reflect those of the United States Government or any agency thereof, SEAD participating Governments or any agencies thereof, the SEAD Operating Agent or The Regents of the University of California.

Ernest Orlando Lawrence Berkeley National Laboratory is an equal opportunity employer. 


\section{Contents}

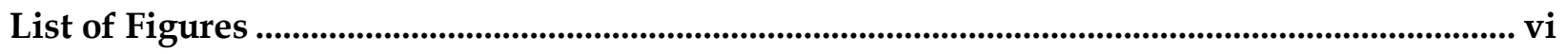

List of Tables....................................................................................................................................viii

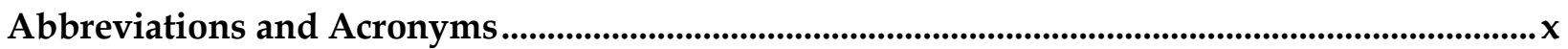

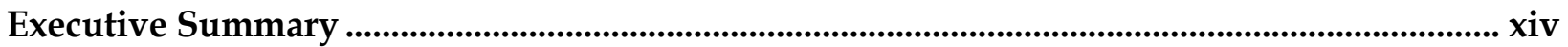

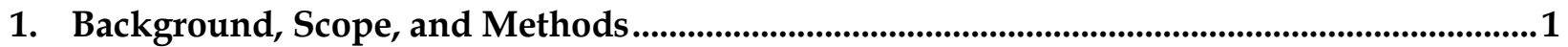

1.1. Super-Efficient Equipment and Appliance Deployment Initiative (SEAD) ............................................... 1

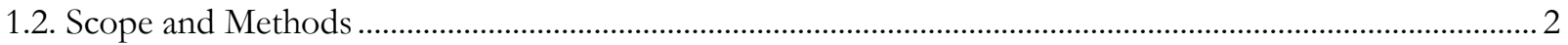

1.2.1. Efficiency Improvement Potentials .................................................................................................. 4

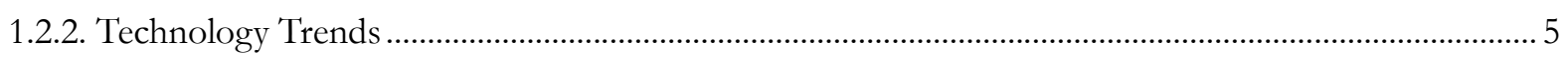

1.2.3. Factors Affecting TV Power Consumption.......................................................................................... 5

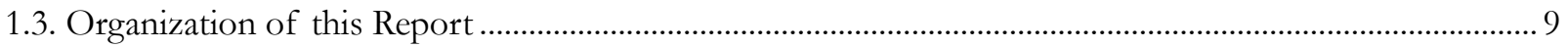

2. TV Market Assessment ........................................................................................................................10

2.1. TV Market Forecast by Technology …………........................................................................................ 10

2.1.1. LCD TV Market Forecast by Backlight Type........................................................................................... 10

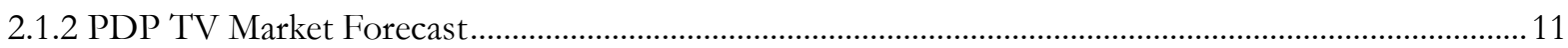

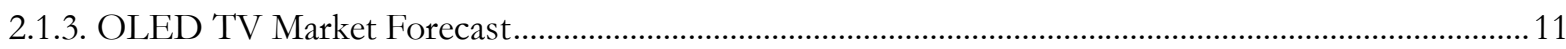

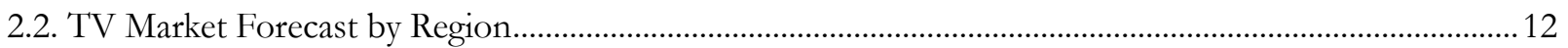

2.2.1. Emerging Market Forecast: India and Brazil........................................................................................ 16

2.2.2. Market Forecast by Country.................................................................................................................... 17

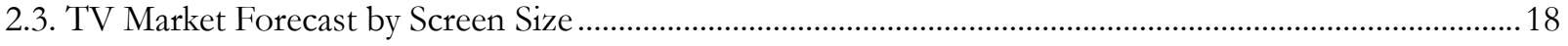

2.3.1. Screen Size Increase and TV Power Consumption................................................................................ 19

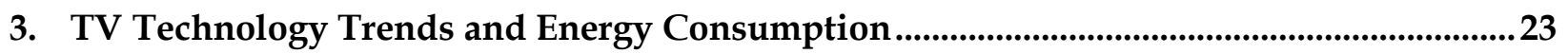

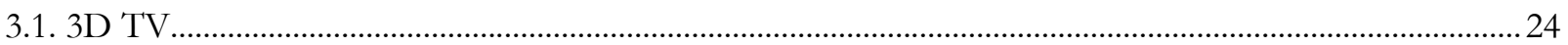

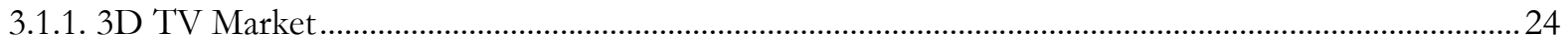

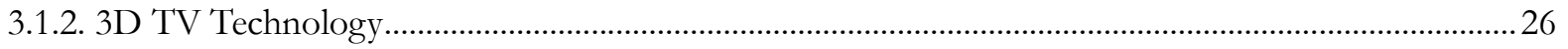

3.1.3. 3D TV Energy Consumption and Efficiency Improvement Potentials ................................................27

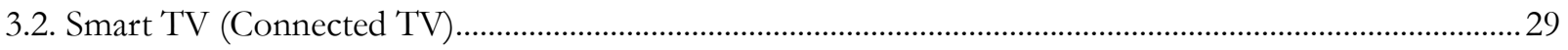

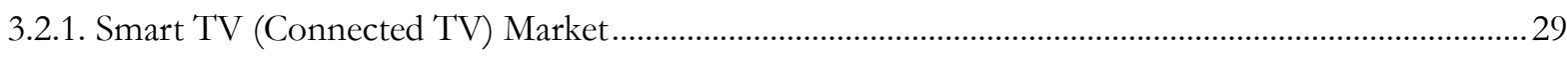

3.2.2. Smart TV (Connected TV) Energy Consumption............................................................................29

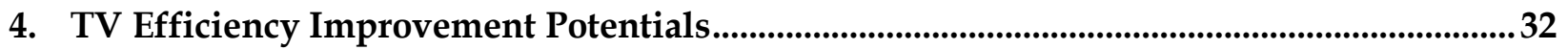

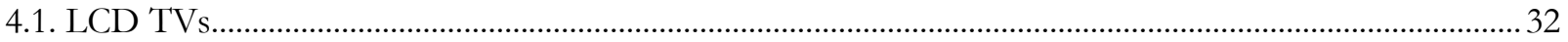

4.1.1. Key Factors Related to Efficiency Improvement in LCD TVs............................................................. 32

4.1.2. Technology Options for Efficiency Improvement of LCD TVs .........................................................33

4.1.3. Summary of Efficiency Improvement Options in LCD TVs...........................................................51 


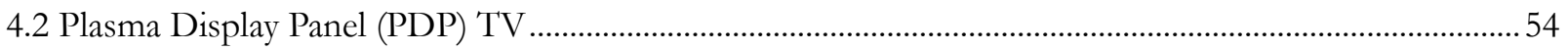

4.2.1. Key Factors Related to Efficiency Improvement in PDP TVs .........................................................5

4.2.2. Technology Options for Efficiency Improvement in PDP TVs ........................................................... 57

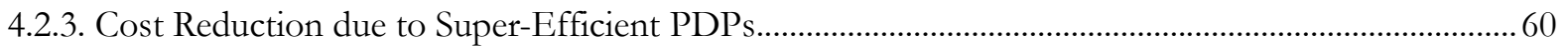

4.2.4. Summary of Efficiency Improvement Options in PDP TVs .............................................................61

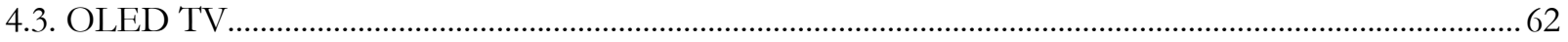

4.3.1. Power Consumption of OLED TVs.................................................................................................. 62

4.3.2. Technology Options for Efficiency Improvements in OLED TVs ....................................................62

4.3.3. Comparison of Production Cost: OLED TV and LCD TV............................................................65

5. Estimates of Energy Savings Potentials (BUENAS) .................................................................66

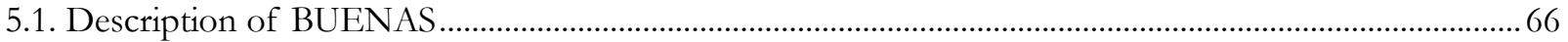

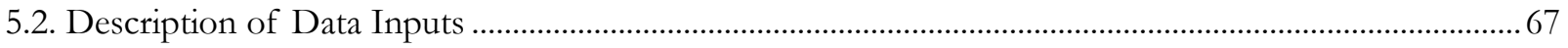

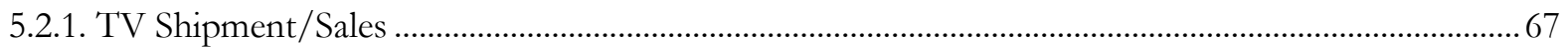

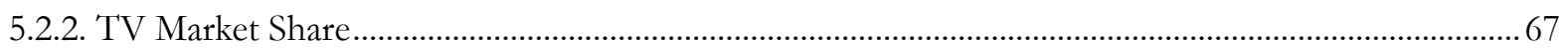

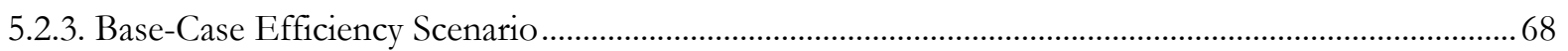

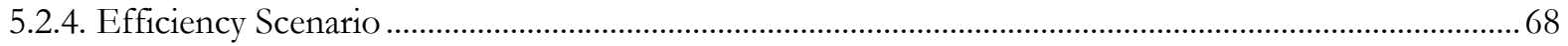

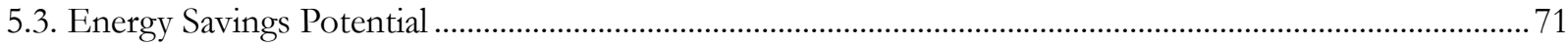

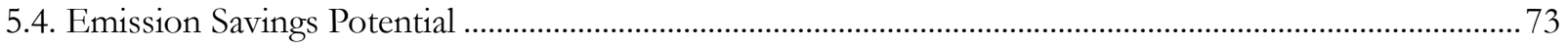

6. Other Issues Related to Power Consumption and Efficiency .................................................... 74

6.1. On-Mode Power and Rated Power ................................................................................................................ 74

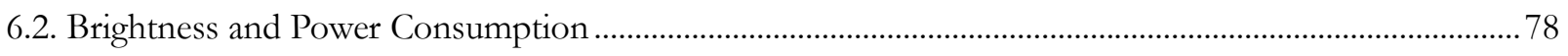

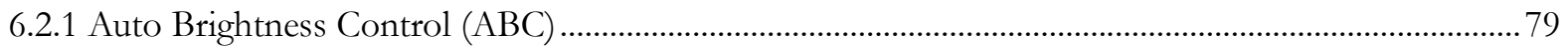

6.3. Technological Learning (Learning by Doing) .............................................................................................. 85

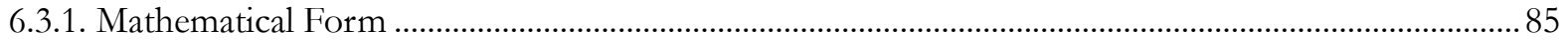

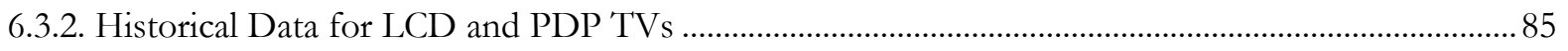

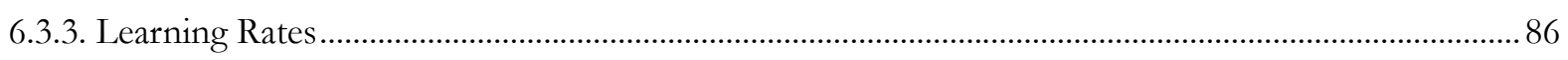

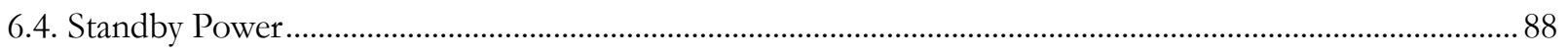

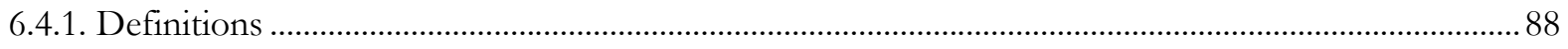

6.4.2. Standby Mode (Sleep Mode) Power of TVs ............................................................................................ 90

6.4.3. Recent Trends of Standby Power in TVs .......................................................................................... 93

6.4.4. Efficiency Improvement Options in Network Standby and Fast Start Mode .................................... 98

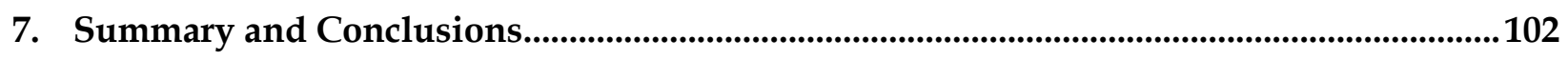

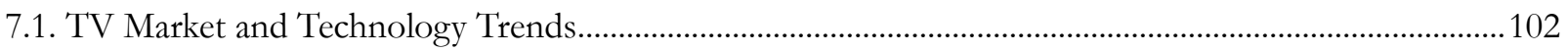

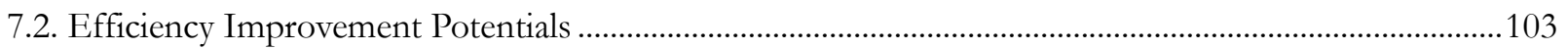

7.3. Energy Consumption and Savings Potentials .......................................................................................... 106

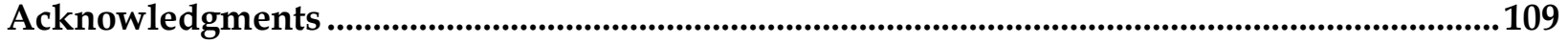




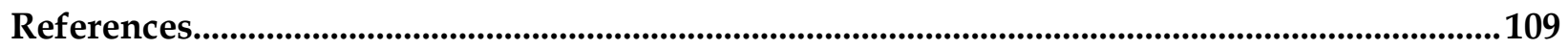

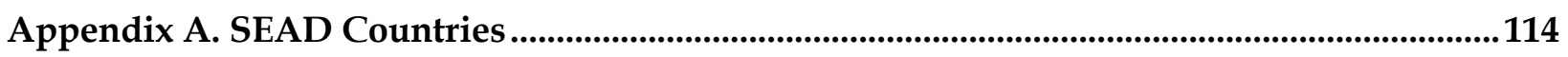

Appendix B. Data, Methodology and Assumptions ..................................................................115

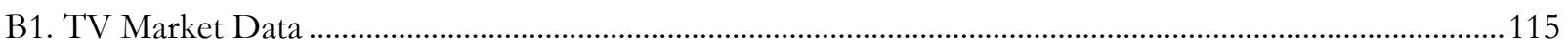

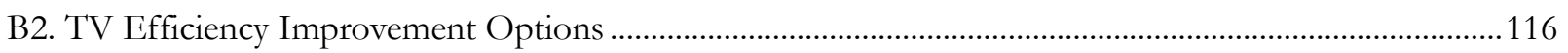

Appendix C. Sensitivity Analysis of Energy-Efficiency Improvement Options ........................124

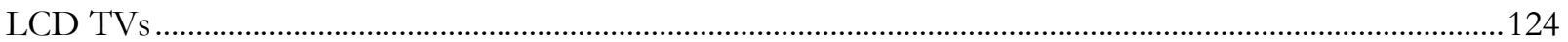

Appendix D. Cost-Efficiency Relationship........................................................................................127

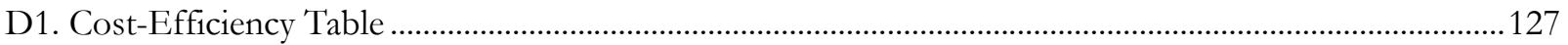

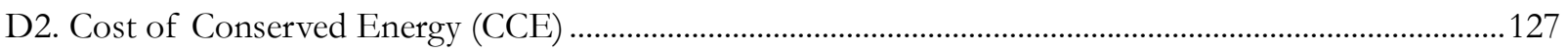

Appendix E. Brightness and Luminance ...............................................................................................129 


\section{List of Figures}

Figure ES-1. Forecast of TV Electricity Consumption in Selected Countries ..................................................... xvii

Figure ES-2. Forecast Global TV Market Transition............................................................................................. xviii

Figure ES-3. Global TV Energy Consumption by Display Type in 2012 ...........................................................iii

Figure ES-4. Forecast Market Transition by Region and Backlight Technology........................................ xix

Figure ES-5 Estimated On-Mode Power for Display Technologies (2012, 42-inch TVs)...................................xx

Figure ES-6. Forecast of TV Electricity Savings Potential in Selected Countries.................................................xi

Figure ES-7. Global TV Shipment and Average Screen Area by Technology in 2010 and 2014 .....................xxii

Figure ES-8. Estimates of Total On-mode Power Consumption by Global TV Shipment in 2014 ................xxii

Figure ES-9. Effect of Predicted Screen Size and TV Shipments on Energy Consumption ..........................xxiv

Figure ES-10. Estimates of Energy Consumption in Network Standby Mode by Global Connected TV

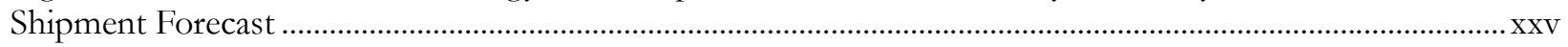

Figure ES-11. Regional Distribution of Global TV Market ................................................................................xxvi

Figure ES-12. On Mode Power of ENERGY STAR TVs with ABC enabled and with ABC disabled.........xxvii

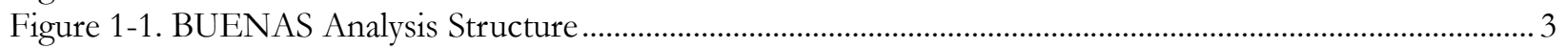

Figure 1-2. Data Analysis Structure .............................................................................................................. 3

Figure 1-3. Global Average TV Screen Size (diagonal) and Total Screen Area Forecast ....................................... 8

Figure 2-1. Global TV Shipment and Screen Size Forecast by Technology ........................................................ 10

Figure 2-2. LCD Backlight Migration by Screen Size .......................................................................................... 11

Figure 2-3. Global TV Shipment by Screen Size ………................................................................................. 11

Figure 2-4. Average Forecast Market Price for 40-inch OLED TVs .................................................................... 12

Figure 2-5. Regional Distribution of Global TV Market from 2009 to 2014 ........................................................13

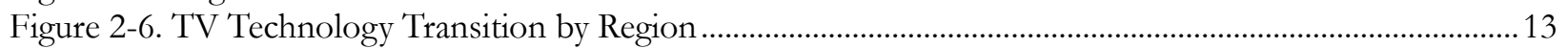

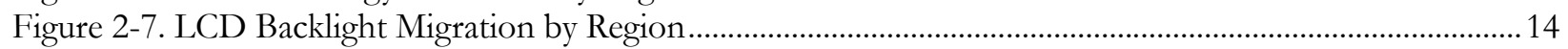

Figure 2-8. CRT and LCD TVs Market Price Forecast Based on U.S. models....................................................14

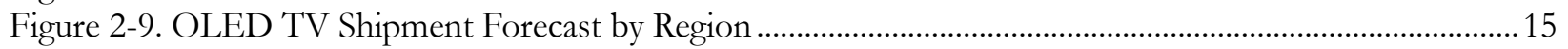

Figure 2-10. India TV Shipment Forecast by Technology ………….................................................................. 16

Figure 2-11. Brazil TV Sales Forecast by Technology ..................................................................................... 16

Figure 2-12. TV Shipment (or Sales) Forecast for SEAD-14 and China ................................................................. 18

Figure 2-13. Screen Size Trend by Region ..................................................................................................... 19

Figure 2-14. Global TV Shipments and Average Screen Area by Technology for 2010 and 2014 ....................19

Figure 2-15. Total On-Mode Power by TV Market Forecast without Efficiency Improvement .........................20

Figure 2-16. Total On-Mode Power by TV Market Forecast with Efficiency Improvement..............................21

Figure 2-17. Effect of Forecast Screen Size and North America TV Shipments on Energy Consumption.....22

Figure 3-1. Stereoscopic Technologies for 3D TV ............................................................................................ 27

Figure 3-2. Estimates of Annual Energy Consumption in 3D mode of 3D TVs by forecasted Global TV

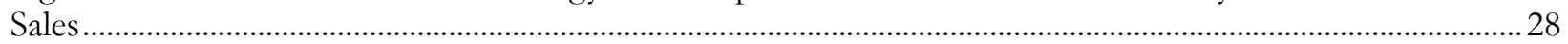

Figure 3-3. Example of Possible Smart TV Size Changes..................................................................................... 30

Figure 4-1. Average Watts per Square Inch of LCD TVs by Backlight Type ..................................................... 34

Figure 4-2. Average U.S. Retail Price and Manufacturing Cost for 32-inch LCD TVs (Q2 2010) ...................... 34

Figure 4-3. LCD Module Manufacturing Costs for 32-inch LCD TVs (Q2 2010) ................................................35

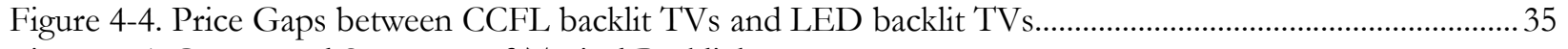

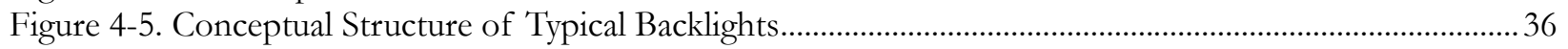

Figure 4-6. LED Roadmap for Luminous Efficacy $(\mathrm{lm} / \mathrm{W})$................................................................................ 37

Figure 4-7. Example of LED Structure with Multiple Quantum Wells (MQWs) ................................................ 38

Figure 4-8. Haitz's Law of LED Light Output and Cost......................................................................................4

Figure 4-9. LED Efficacy Improvement $(\mathrm{lm} / \mathrm{W})$ and BLU Power Consumption (W) .......................................41

Figure 4-10. Brightness Enhancement Film (BEF) and Brightness Gain of Various Lens Films .................... 42

Figure 4-11. Utilization of Light With and Without a DBEF.............................................................................4

Figure 4-12. Mechanism of Micro-lens Films.................................................................................................... 43 


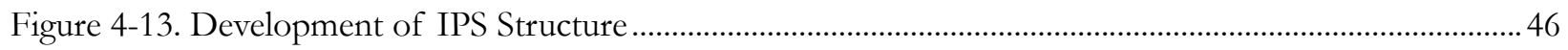

Figure 4-14. Global Dimming and Local Dimming........................................................................................... 49

Figure 4-15. Cost-effective Options for 32-inch Entry-level LED-LCD TVs .....................................................52

Figure 4-16. Estimates of On-mode Power for 32-inch LED Backlit TVs .........................................................53

Figure 4-17. Power Reduction by Luminous Efficacy Improvement......................................................................55

Figure 4-18. On-Mode Power of Panasonic PDP TVs .......................................................................................... 56

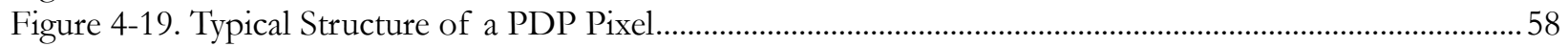

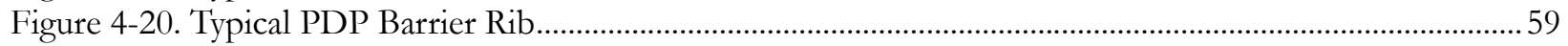

Figure 4-21. Indexed 42-inch HD Plasma Panel Costs at $2.5 \mathrm{~lm} / \mathrm{W}$ and $5.0 \mathrm{~lm} / \mathrm{W}$..........................................60

Figure 4-22. Forecast for On-Mode Power Consumption of 42-inch PDP TVs ................................................61

Figure 4-23. Power Consumption Roadmap for 40-inch (101.6-cm) OLED TV Panels......................................63

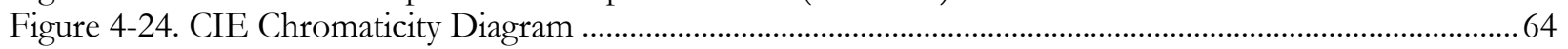

Figure 4-25. Forecast for On-Mode Power of a 32-inch OLED TV .................................................................. 64

Figure 4-26. Forecast OLED TV Production Cost and Average Market Price.........................................................65

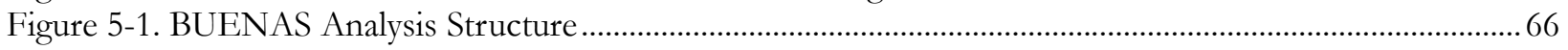

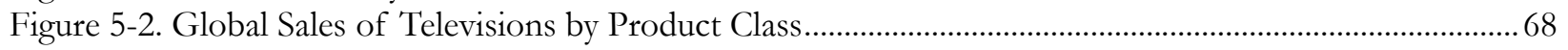

Figure 5-3. Global Consumption of TVs by Product class .................................................................................... 71

Figure 5-4. Energy Consumption of TVs in Selected Countries.......................................................................... 71

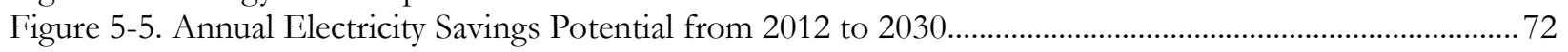

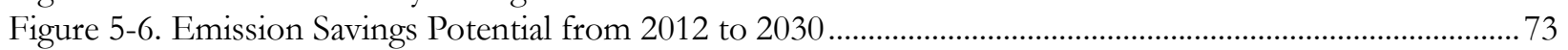

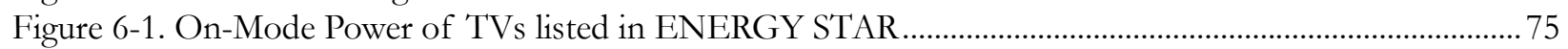

Figure 6-2. On-Mode Power and Rated Power of LCD TVs (Japan) ....................................................................... 76

Figure 6-3. On-Mode Power and Rated Power of PDP TVs (Japan) …………….............................................76

Figure 6-4. On-Mode and Rated Power of 32-inch LCD and 42-inch PDP TVs (Japan)...................................77

Figure 6-5. Power Consumption of a 46-inch LED-LCD TV in Various Modes ....................................................78

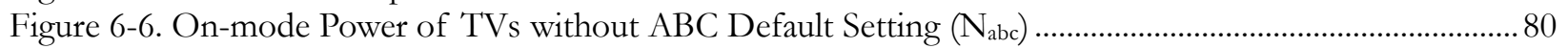

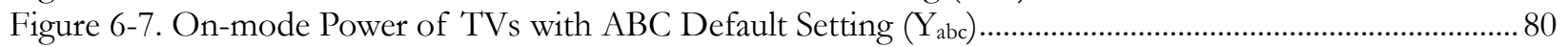

Figure 6-8. Distribution of ENERGY STAR-qualified LCD TVs by ABC Setting.............................................81

Figure 6-9. On-mode Power of ENERGY STAR-qualified TVs with ABC Disabled......................................... 82

Figure 6-10. Effect of ABC Setting on Average On-mode Power of ENERGY STAR TVs............................. 83

Figure 6-11. On-mode Power of 32-inch LCD TVs with ABC and without ABC ............................................ 83

Figure 6-12. On-mode Power of 40-inch LCD TVs .......................................................................................... 84

Figure 6-13. On-mode Power of PDP TVs ........................................................................................................ 84

Figure 6-14. Price Learning Rates for LCD and PDP TVs (2007 to 2010) ........................................................... 87

Figure 6-15. Price Learning Rates Estimated for LCD and PDP TVs (2007 to 2014) .....................................87

Figure 6-16. Distribution of Sleep Mode Power of ENERGY STAR-qualified LCD and PDP TVs............... 91

Figure 6-17. Sleep Mode Power of ENERGY STAR-qualified TVs by Screen Area .........................................91

Figure 6-18. Average Sleep Mode Power of TVs in Japan and the U.S. ............................................................. 92

Figure 6-19. Estimates of Energy Consumption in Sleep Mode by Global TV Sales ........................................ 92

Figure 6-20. Estimates of Energy Consumption in Fast Start Mode by Global TV Sales.................................99

Figure 6-21. Estimates of Energy Consumption in Data Acquisition Mode by Global TV Sales...................... 95

Figure 6-22. Estimates of Energy Consumption in Network Standby Mode by Global Connected TV Sales 98

Figure 6-23. Startup Time and Resource Utilization of Digital TV System ........................................................100

Figure 7-1. Cost-Efficiency Relationships in FPD Technologies.........................................................................106

Figure C-1. UEC Sensitivity to Technical Factors (32" LED-LCD TV, $P_{\text {network standby }}=4 \mathrm{~W}$ ) ............................125

Figure C-2. UEC Sensitivity to Technical Factors (32” LED-LCD TV, $\mathrm{P}_{\text {network standby }}=10 \mathrm{~W}$ ).........................125

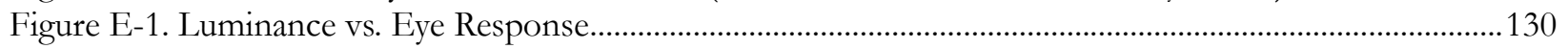




\section{List of Tables}

Table ES-1. Increase in TV Screen Sizes and Shipments for North America ....................................................xxiii

Table ES-2. Summary of Energy Consumption Forecast in TVs ...............................................................................

Table 1-1. Key Factors Affecting TV On-Mode Power ............................................................................................ 6

Table 1-2. Technical Factors that affect TV Reference Power Consumption......................................................... 6

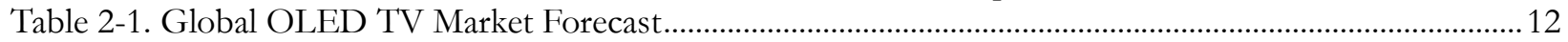

Table 2-2. New Design Trends in Entry-level LED-LCD TVs .......................................................................... 15

Table 2-3. Estimated 2010 TV Shipments (or Sales) for SEAD-14 and China ...................................................... 17

Table 2-4. Average Screen Area per Unit and Average On-Mode Power for Technologies without Efficiency

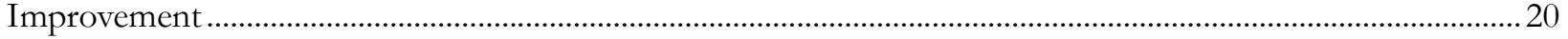

Table 2-5. Average Display Area per Unit and Average On-Mode Power for Technologies with Efficiency

Improvement ..................................................................................................................................................22

Table 2-6. Increase in TV Power Consumption Corresponding to Predicted Increase in Screen Size ..............21

Table 2-7. Increase in Screen Size and TV Shipments for North America...........................................................22

Table 3-1. Effect of 3D TV and Smart TV on Unit Efficiency and Energy Consumption ..............................24

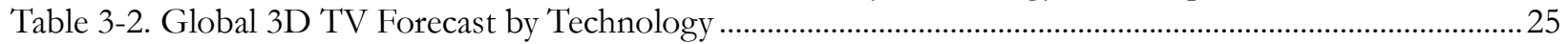

Table 3-3. Global 3D TV Market Penetration by Technology and Screen Size ...................................................25

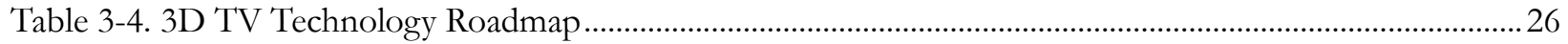

Table 3-5. Example of Light Loss in 3D mode of 3D LCD TV with Shutter Glasses......................................27

Table 3-6. Connected TV Market Forecast..............................................................................................................29

Table 3-7. Example of Specifications for Image Processing Circuitry in Smart TV versus Conventional TV. 30

Table 4-1. Example of Change in Luminance for a Typical LCD TV Set............................................................33

Table 4-2. Comparison of 46-inch (904-cm) LCD TVs by Backlight Type ............................................................36

Table 4-3. LED Backlight Specifications for 32-inch (81.3-cm) LCD TVs ............................................................ 37

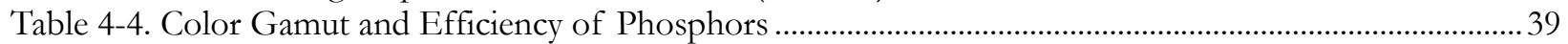

Table 4-5. Effect of LED Improvement on Power Consumption, 32-inch LCD TVs ....................................... 39

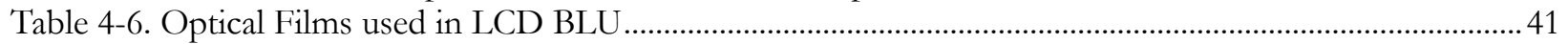

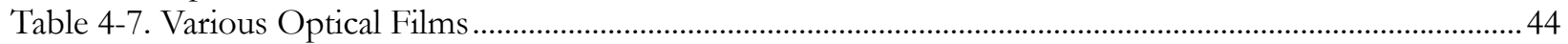

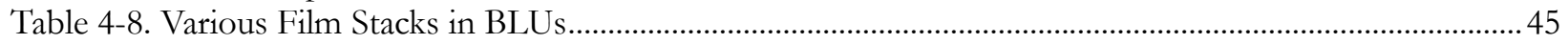

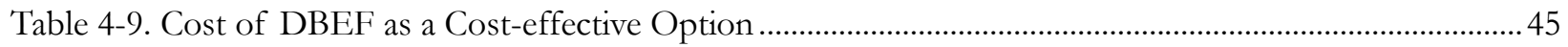

Table 4-10. Forecast of Average LCD Panel Transmittance for TVs ..................................................................4

Table 4-11. Effect of Panel Transmittance on LED Backlight Power Consumption .........................................47

Table 4-12. Average Effect of Dynamic Dimming on Power Reduction in LED Backlit TVs.......................... 49

Table 4-13. Structures of LED-edge Backlight and Dimming Methods..............................................................50

Table 4-14. Incremental Cost of Dimming Options ...........................................................................................51

Table 4-15. Example of Efficiency Improvement Forecast for 32-inch LCD TVs .............................................51

Table 4-16. Luminance of a 42-inch (106.7-cm) HD PDP TV ………….............................................................. 54

Table 4-17. 42-inch (106.7-cm) HD PDP TVs (ENERGY STAR 2010) ………………………………............55

Table 4-18. PDP Screen Size and Cell Pitch (not to scale) ………………...........................................................56

Table 4-19. Commercially Available PDP TV Screen Resolution and Aspect Ratio ..............................................57

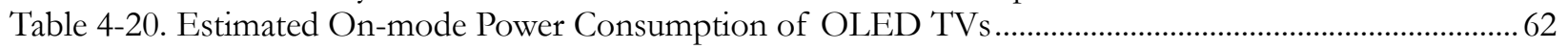

Table 5-1. TV Shipment/Sales by Country and Growth Rates...............................................................................67

Table 5-2. Efficiency and Global Market Shares by Technology in the Base Case .................................................68

Table 5-3. Efficiency and Global Market Shares by Technology in the Efficiency Scenario ...............................69

Table 5-4. TV Usage and Energy Consumption by Country …………………………………............................ 70

Table 5-5. Annual/Cumulative Electricity Savings Potential for 2015, 2020, and 2030 …..................................72

Table 5-6. Annual Emission Savings Potential for 2015, 2020, and 2030 ..........................................................73

Table 6-1. Luminance of 32-inch ENERGY STAR TVs in Default and Brightest Modes .................................79

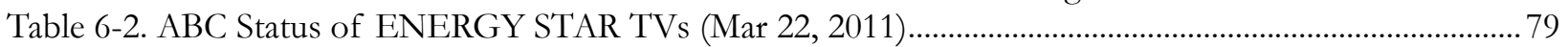

Table 6-3. Average Market Price of LCD and PDP TVs in the U.S., $1^{\text {st }}$ Quarter 2010 ........................................ 86 


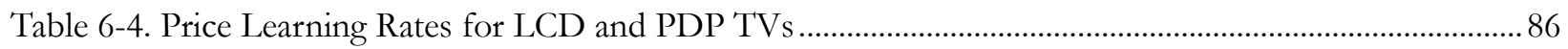

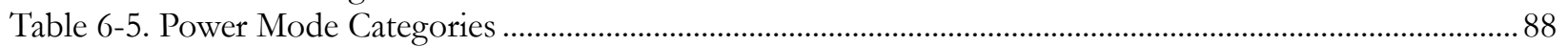

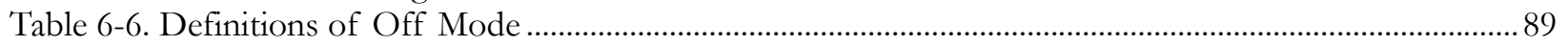

Table 6-7. Definitions of Active Standby and Passive Standby............................................................................ 90

Table 6-8. Estimates of TV Unit Energy Consumption (UEC) in On-Mode and Standby Mode.....................93

Table 6-9. Power Consumption in Fast Start Mode of TVs.................................................................................93

Table 6-10. Power Consumption in Data Acquisition Mode of TVs ................................................................95

Table 6-11. Connected TV Market Forecast and Network Standby Power ........................................................... 97

Table 7-1. Summary Table of Power Consumption in Various Modes of TVs..................................................107

Table 7-2. Summary Table of Energy Consumption Forecast in TVs ...............................................................108

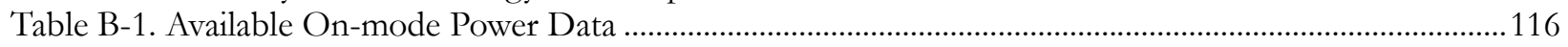

Table B-2. Volume-weighted EEI for LED backlit TVs by Regions ................................................................117

Table B-3. Average On-mode Power of Screen Technologies for Each Country in 2010 ...............................117

Table B-4. Efficiency Scenarios applied to BUENAS model ..................................................................................118

Table B-5. Average Screen Size (inches) for each Region in 2010 by Technology ..............................................118

Table B-6. Estimates of "On," "Standby," and "Off" times for TVs ..................................................................120

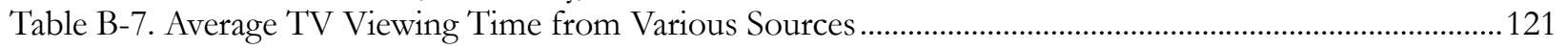

Table D-1. Cost-Efficiency Table for LCD TVs ...............................................................................................127

Table E-1. Concepts Relevant to Luminance ......................................................................................................... 129 


\section{Abbreviations and Acronyms}

\begin{tabular}{|c|c|}
\hline $\mathrm{ABC}$ & automatic brightness control \\
\hline AP & Asia Pacific \\
\hline APL & average picture level \\
\hline ARP & address resolution protocol for internet protocol version 4 (IPv4) \\
\hline ATSC & Advanced Television System Committee \\
\hline $\mathrm{A} / \mathrm{V}$ & audio/video \\
\hline BEF & brightness enhancement film \\
\hline BLU & backlight unit \\
\hline BUENAS & Bottom-Up Energy Analysis System \\
\hline $\mathrm{CaO}$ & calcium oxide \\
\hline CCFL & cold cathode fluorescent lamp \\
\hline $\mathrm{cd}$ & candela \\
\hline $\mathrm{cm}$ & centimeter \\
\hline $\mathrm{cm}^{2}$ & square centimeter \\
\hline $\mathrm{CPU}$ & central processing unit \\
\hline CRT & cathode ray tube \\
\hline DAM & download acquisition mode \\
\hline DBEF & dual brightness enhancement film \\
\hline DLNA & Digital Living Network Alliance \\
\hline DOE & United States Department of Energy \\
\hline DVD & digital video (versatile) disc \\
\hline $\mathrm{EE}$ & Eastern Europe \\
\hline EEI & energy efficiency index \\
\hline EPG & electronic program guide \\
\hline EQE & external quantum efficiency \\
\hline ERC & energy recovery circuit \\
\hline
\end{tabular}




\begin{tabular}{|c|c|}
\hline $\mathrm{EU}$ & European Union \\
\hline FHD & full high definition \\
\hline FPD & flat panel display \\
\hline GPU & graphical processing unit \\
\hline HD & high definition \\
\hline HDMI & high definition multimedia interface \\
\hline HDMI CEC & HDMI consumer electronics control \\
\hline $\mathrm{Hz}$ & Hertz \\
\hline IC & integrated circuit \\
\hline IEEE & Institute for Electrical and Electronic Engineers \\
\hline IMID & International Meeting on Information Display \\
\hline $\mathrm{in}^{2}$ & square inch \\
\hline IPS & in-plane switching \\
\hline IQE & internal quantum efficiency \\
\hline ECCJ & Energy Conservation Center, Japan \\
\hline KWh & kilowatt hour \\
\hline LA & Latin America \\
\hline LAN & local area network \\
\hline LC & liquid crystal \\
\hline LCD & liquid crystal display \\
\hline LED & light-emitting diode \\
\hline LGP & light guide panel \\
\hline $\operatorname{lm} / \mathrm{W}$ & lumens per Watt \\
\hline LPI & low-power idle \\
\hline ME\&A & Middle East and Africa \\
\hline $\mathrm{MgO}$ & magnesium oxide \\
\hline $\mathrm{MoCA}$ & Multimedia over Coax Alliance \\
\hline MQW & multiple quantum wells \\
\hline MTP & market transformation program \\
\hline NA & North America \\
\hline
\end{tabular}




\begin{tabular}{|c|c|}
\hline $\mathrm{nm}$ & nanometer \\
\hline NTSC & National Television System Committee \\
\hline OECD & Organization for Economic Cooperation and Development \\
\hline OLED & organic light-emitting diode \\
\hline OS & operating system \\
\hline $\mathrm{p}$ & progressive \\
\hline$P_{\text {basic }}$ & basic power consumption \\
\hline $\mathrm{P}_{\text {display }}$ & display power consumption \\
\hline $\mathrm{P}_{\text {screen }}$ & screen power consumption \\
\hline PC & personal computer \\
\hline PDP & plasma display panel \\
\hline PHOLED & phosphorescent organic light-emitting diode \\
\hline PSU & power supply unit \\
\hline $\mathrm{R} \& \mathrm{D}$ & research and development \\
\hline RAM & random access memory \\
\hline RP TV & rear projection $\mathrm{TV}$ \\
\hline SEAD & super-efficient equipment and appliance deployment \\
\hline $\mathrm{SiN}_{2}$ & silicon nitride \\
\hline $\mathrm{SiO}_{2}$ & silicon dioxide \\
\hline S-IPS & super in-plane switching \\
\hline $\mathrm{SrO}$ & strontium oxide \\
\hline TFT & thin-film transistor \\
\hline $\mathrm{TN}$ & twisted nematic \\
\hline TV & television \\
\hline TWh & terawatt hour \\
\hline UEC & unit energy consumption \\
\hline UV & ultraviolet \\
\hline UPnP & universal plug and play \\
\hline VA & vertical alignment \\
\hline W & Watt \\
\hline
\end{tabular}


WAN wide area network

WLAN wide local area network

WE Western Europe

WOL wake on LAN

WoWLAN wake-on-wireless LAN

Xe xenon 


\section{Executive Summary}

This report presents the results of an analysis, commissioned by the U.S. Department of Energy, of television (TV) efficiency in support of the Super-efficient Equipment and Appliance Deployment (SEAD) initiative. ${ }^{1}$ The International Energy Studies group at Lawrence Berkeley National Laboratory performed the analysis. SEAD aims to transform the global market by increasing the penetration of highly efficient equipment and appliances. The objective of this analysis is to provide the background technical information necessary to improve the efficiency of TVs and to provide a foundation for the voluntary activities of SEAD participating countries.

SEAD partners work together in voluntary activities to: (1) "raise the efficiency ceiling" by pulling super-efficient appliances and equipment into the market through cooperation on measures like incentives, procurement, awards, and research and development (R\&D) investments; (2) "raise the efficiency floor" by working together to bolster national or regional policies like minimum efficiency standards; and (3) "strengthen the efficiency foundations" of programs by coordinating technical work to support these activities. ${ }^{2}$

\section{Objective and Scope}

The objective of this analysis is to identify potential TV efficiency improvements and their incremental costs, as well as to provide initial global and country-specific estimates of total energy savings potential. The overarching goal is to provide relevant and appropriate information to support design of appropriate policy programs that will accelerate the penetration of super-efficient TVs.

This report defines three categories of potential TV efficiency improvement: market, economic, and technical. The analysis addresses market and economic efficiency improvements that are technically feasible, practical to manufacture, and therefore could be realized in the short term. In addition, we discuss significant technology trends to provide a picture of the future TV market and analyze technical improvements that are feasible in the short term, but we do not analyze in detail long-term technical efficiency improvements that would require R\&D investment.

\section{Data Sources and Analysis Method}

The analysis team obtained the data for this report from the following sources: review of literature including technical reports; country-specific databases (i.e., U.S. ENERGY STAR and Energy Conservation Center, Japan); international conferences and exhibitions; and interviews with

\footnotetext{
1 As one of the initiatives in the Global Energy Efficiency Challenge, SEAD seeks to enable high-level global action by informing the Clean Energy Ministerial dialogue. In keeping with its goal of achieving global energy savings through efficiency, SEAD was approved as a task within the International Partnership for Energy Efficiency Cooperation (IPEEC) in January 2010.

2 As of April 2011, the governments participating in SEAD are: Australia, Brazil, Canada, the European Commission, France, Germany, India, Japan, Korea, Mexico, Russia, South Africa, Sweden, the United Arab Emirates, the United Kingdom, and the United States. More information on SEAD is available from its website at http://www.superefficient.org/.
} 
manufacturers and experts in the field. The experts and manufacturers interviewed have many years' experience in the TV industry, and their collective expertise covers the entire TV market, including liquid crystal display (LCD), plasma display panel (PDP), and organic light-emitting diode (OLED) technologies, research and development (R\&D), planning, and TV testing. The identities of the expert and manufacturer sources interviewed for this report have been kept confidential because they requested anonymity as a condition of speaking with authors. Information from these interviews in the text of this report is, therefore, simply attributed to "experts" and/or "manufacturers."

Our analysis compares future TV energy consumption for two scenarios: a base case, which assumes options that are expected to be implemented from manufacturers' roadmaps, and an efficiency case, which assumes all cost-effective efficiency options that can be additionally implemented if some

incremental costs and technical effort are expended. Some efficiency improvement options needing further R\&D investment or currently unfeasible are discussed but not included in our modeling.

\section{Summary of Findings and Recommendations}

\section{TV Market}

- LCD TVs are rapidly displacing CRT TVs, and light-emitting diode (LED) backlit LCD TVs are similarly rapidly displacing cold cathode fluorescent lamp (CCFL) backlit LCD TVs. As a result, LED backlit TVs are expected to capture more than $75 \%$ of the global TV sales in 2014. The transition from analog to digital TV, new energy-efficiency standards and improvements and cost reductions in LED technology will be the key drivers for these technology transitions.

- The large-scale transition from CCFL backlit LCD TVs to LED backlit LCD TVs between 2010 and 2014 is expected to reduce the impact of increases in screen size and TV sales on total TV power consumption even in the absence of further efficiency improvements in LED backlit TVs.

- The top five manufacturers produce more than $60 \%$ of TVs worldwide.

- Some major TV brands are expected to provide more efficient LED backlit TVs at lower prices through adjusting the maximum luminance level and color-reproduction capability.

- The Japanese domestic market showed significant sensitivity to the government's Eco-points program, growing more than 30\% in 2009 and 2010.

\section{TV Energy Consumption and Efficiency Improvement Potentials}

- The average LED backlit LCD TV in the US market is forecasted to consume about $20 \%$ less on-mode power than ENERGY STAR Version 5 requirements (for 32"-42" models) in 2012.

- Key cost-effective options exist that can further improve efficiency (Cost of Conserved Energy $<8$ cents $/ \mathrm{kWh}$ ).

- All the efficiency improvement options considered also apply to 3D TVs and connected TVs (or smart TVs). 
- Brightness control functions play a significant role in controlling a TV's on-mode power. However, currently available TVs still consume more power in various modes (e.g., dynamic and vivid) other than default home mode.

- ENERGY STAR Version 5 is likely to hold TV energy consumption down in spite of growth in global TV sales and a trend toward increased screen sizes.

- Connected TVs (or smart TVs) are expected to consume $3 \mathrm{~W}$ to $30 \mathrm{~W}$ in network standby, depending on manufacturers' internal design schemes. Various options exist to keep overall network standby power low; including network standby power in TV energy test procedures would be the most important step in reducing power consumption.

- Idle modes such as "fast play", "quick start" can contribute about $25 \mathrm{~W}$ on average to standby power consumption as a user selected option. To the extent that fast boot time (or reactivation time) is an important choice for consumers, market transformation program administrators might consider efforts to educate consumers regarding the significant energy consumption implications of such choices.

\section{Analysis Results}

There is significant uncertainty regarding precisely which efficiency improvement options TV manufacturers will gravitate toward in future TV designs. Therefore, although this report reviews and analyzes currently available and dominant technology in some detail in order to identify feasible and cost-effective efficiency improvement options, we do not claim these are the best or only efficiency improvement options available for the various TV technologies, or that other cost effective options to improve efficiency do not exist. Discussion of specific technologies is not intended to be exhaustive and comprehensive, only to provide policy makers with a sense of what levels of efficiency are possible for televisions currently and in the next 2 to 5 years. We do not recommend or endorse any specific technology or efficiency improvement option.

\section{A] Trends in TV Electricity Consumption}

Currently, TVs are estimated to represent more than $3 \%$ to $4 \%$ of global residential electricity consumption ( $\sim 168$ terrawatt hours [TWh], representing $\sim 27$ megatonnes of $\mathrm{CO}_{2}$ emissions in 2010) ${ }^{3}$. As shown in Figure ES-1, TV electricity consumption is expected to slightly decrease in the short term, because of a large-scale technological transition (e.g., CRT to LCD, and CCFL-LCD to LEDLCD) and rapid improvements in TV energy efficiency, in spite of the projected increase in penetration of TVs in households, especially in emerging economies, as well as the projected increase in the average screen size of TVs purchased.

\footnotetext{
3 This estimate is based on average on-mode power consumption of ENERGY STAR-qualified TVs in 2010, efficiency improvement potentials identified, and regional TV shares. Expected additional energy consumption in 3D TVs, connected TVs (or smart TVs), various display settings, and power modes are not included in the modeling. These components are separately discussed in the report.
} 


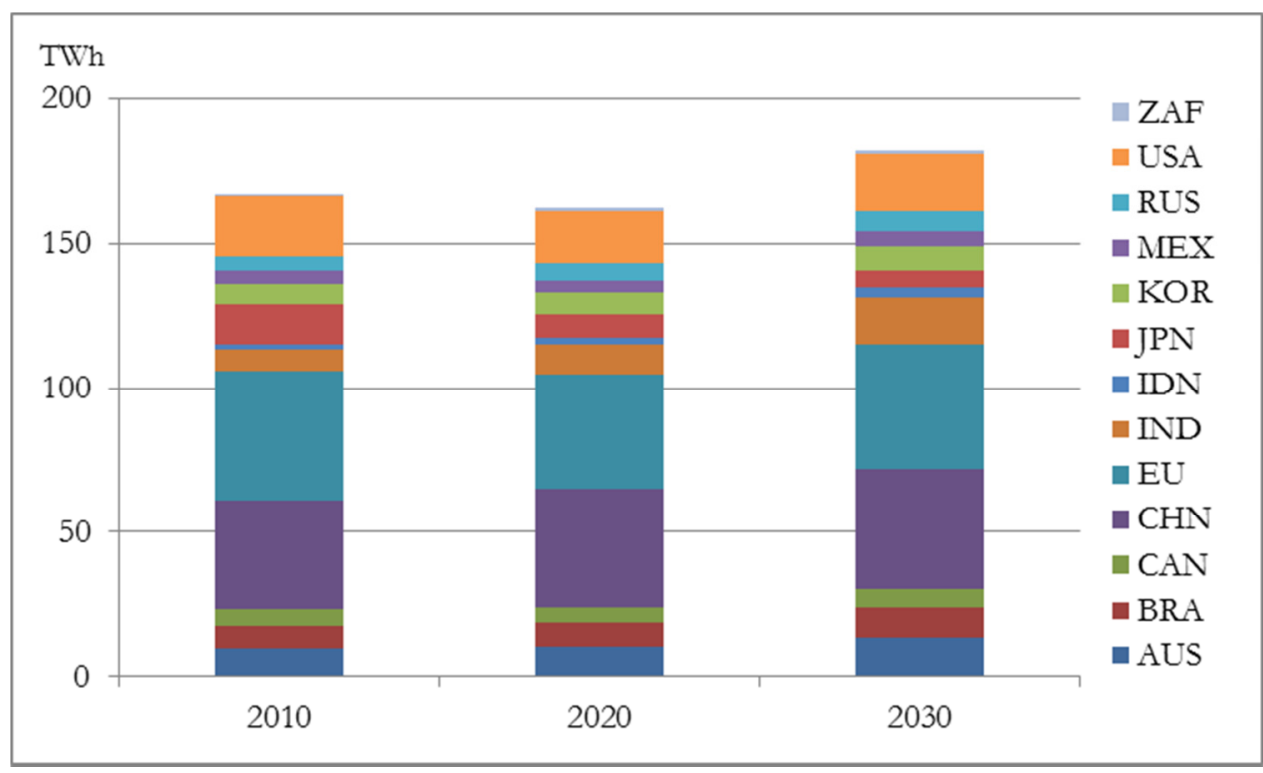

Source: Analysis using the Bottom-Up Energy Analysis System (BUENAS) model

Figure ES-1. Forecast of TV Electricity Consumption in Selected Countries

Assuming that the average TV lifetime ${ }^{4}$, including replacement cycles, is about 10 years, almost all of the TV electricity consumption in 2030 will be from TVs that are yet to be purchased. This presents a great opportunity to influence these purchase decisions and save electricity in a cost-effective manner.

Since the mid-2000s, the global TV market has undergone a major transition from traditional cathode ray tube (CRT) TVs to other types, particularly flat panel display (FPD) TVs such as LCD and PDP. ${ }^{5}$ LCD TVs are expected to account for more than $90 \%$ of the global TV market through 2012, including all screen sizes (DisplaySearch 2011a). Although CRT TVs are expected to remain popular in emerging markets, major TV brands are likely to provide more affordable LCD TVs to replace CRT TVs in these markets. In addition, a large-scale transition is expected from conventional cold cathode fluorescent lamp (CCFL) backlit LCD TVs to light-emitting diode (LED) backlit LCD TVs for all screen sizes, resulting in substantial improvements in efficiency. Figure ES-2 shows the predicted market transition from CRT to LCD, and CCFL-LCD to LED-LCD TVs.

\footnotetext{
4 TV's technical lifespan is different from this value. According to TV manufacturers, TV's technical lifespan is more than 60,000 hours which is equivalent to about 20 years at 8 hours a day.

5 According to DisplaySearch, global shipments of LCD TVs in 2007 were $39.7 \%$ of the global market, CRT TVs $53.7 \%$, PDP TVs $5.6 \%$, and Rear Projection TVs 1\%. (DisplaySearch 2010b)
} 


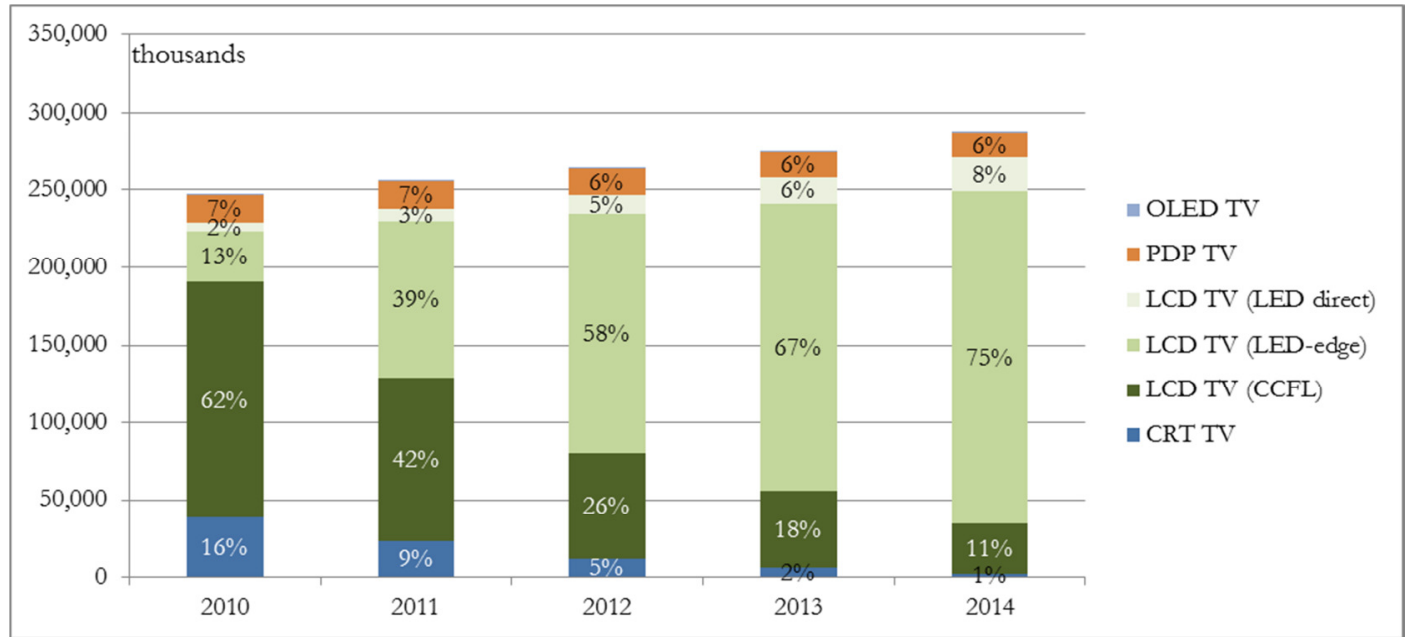

Source: DisplaySearch 2011a

Figure ES-2. Forecast Global TV Market Transition

Reflecting the growing contribution of LCD TVs to global energy consumption due to the aforementioned market transition, we estimate that LCD TVs will overtake CRT TVs in terms of energy consumption consuming 84.3 terawatt-hours (TWh) annually compared to $72.8 \mathrm{TWh}$ for CRT TVs as shown in Figure ES-3.

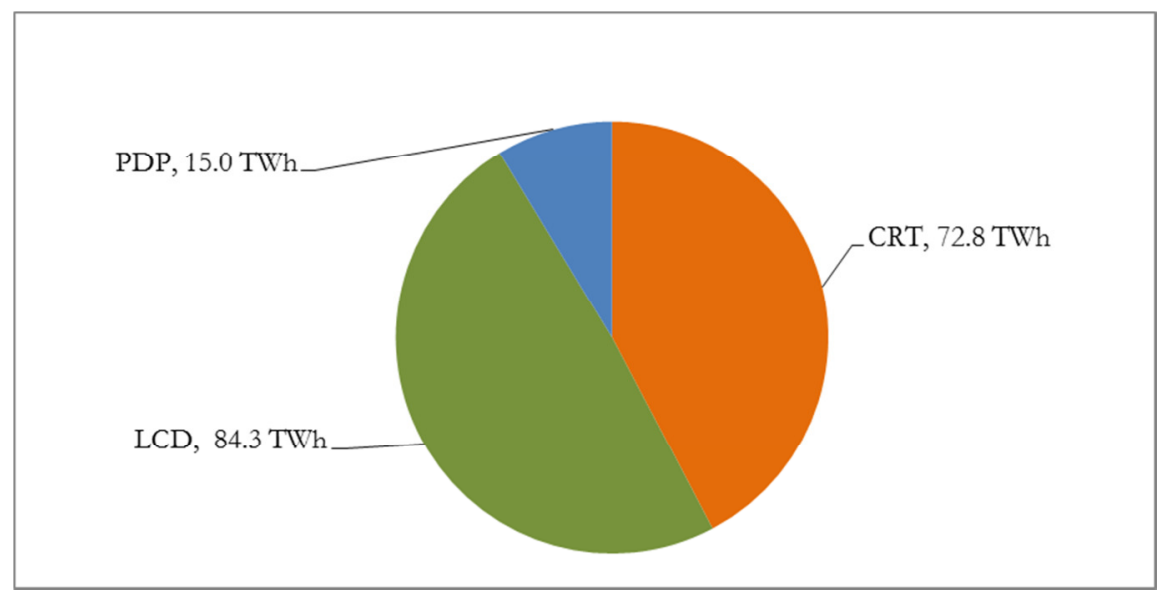

Source: Analysis using the Bottom-Up Energy Analysis System (BUENAS) model

Figure ES-3. Global TV Energy Consumption by Display Type in 2012

\section{Regional Trends}

TV manufacturing is highly globalized and concentrated; the top five manufacturers produce more than $60 \%$ of TVs sold worldwide. There are only limited regional differences in screen technologies and sizes (see Figure ES-4). For a given size and display technology, TVs sold in different regions of the world are very similar. Our analysis covers the most common TV sizes and display technologies found across different regions. 


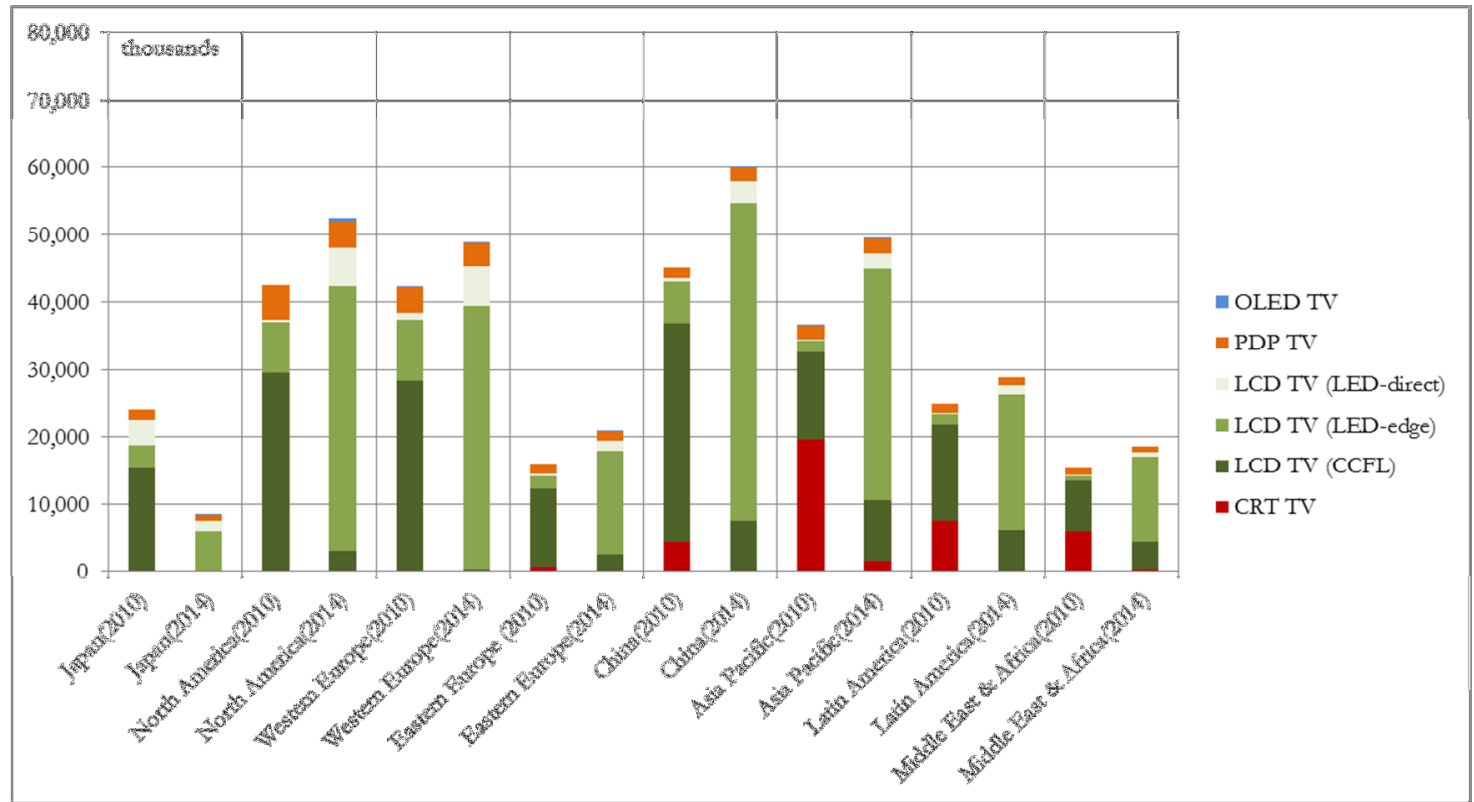

Source: DisplaySearch 2011a

Figure ES-4. Forecast Market Transition by Region and Backlight Technology

Because TV production is highly globalized and TVs sold across different regions are similar, our analysis does not consider separate efficiency options and costs for different regions of the world, while we reflect different screen technology mixtures and TV sales in each region. However, major TV brands are expected to provide newly-designed LED backlit TVs at low prices in emerging markets. This new type of low-price model can be accomplished by lowering the maximum luminance level and color reproduction capability in LCD panels, resulting in lower power consumption than conventional LED backlit LCD TVs.

\section{B] TV On-Mode Power Forecast}

As mentioned earlier, our analysis compares future TV energy consumption for two scenarios: a base case, which assumes options that are expected to be implemented from major manufacturers' roadmaps, and an efficiency case, which assumes cost-effective efficiency options that can be additionally implemented with incremental costs and some technical effort. For LCD TVs, the base case focuses on LED-edge backlit TV models because they are expected to be the mainstream technology. However, improvement in other key parts, such as LCD panels and optical films, can be applicable to other types of backlights. There are only a few technology categories where the relationship between efficiency improvement and corresponding incremental cost is clear. While most panel-related technologies require $\mathrm{R} \& \mathrm{D}$ investment because panel design is the most complicated element and is closely associated with manufacturing process, adoption of better optical films and dimming options appear to be good candidates for a market transformation program. While technical options for improving the efficiency of PDP TVs exist, most such options are interconnected. Therefore it is difficult to quantify the individual effect that each individual option would have on on-mode power. On-mode power forecasts for PDP TVs are based on manufacturers' roadmaps. OLEDs have energy-efficiency improvement potential; these options are 
strongly related to proprietary OLED panel technologies, including sophisticated manufacturing processes. This report does not address R\&D investments associated with design of FPDs.

Based on our identification of efficiency improvement options and on-mode power for ENERGY STAR Version 4 qualified TVs, all TV technologies ( $\leq 50$-inch screen size) are expected to be able to meet or approach ENERGY STAR Version 5 levels in 2012 by employing some combination of efficiency improvement options, as shown in Figure ES-5, although some of these options may require additional incremental costs. The average for LED backlit LCD TVs is about $20 \%$ below ENERGY STAR Version 5.

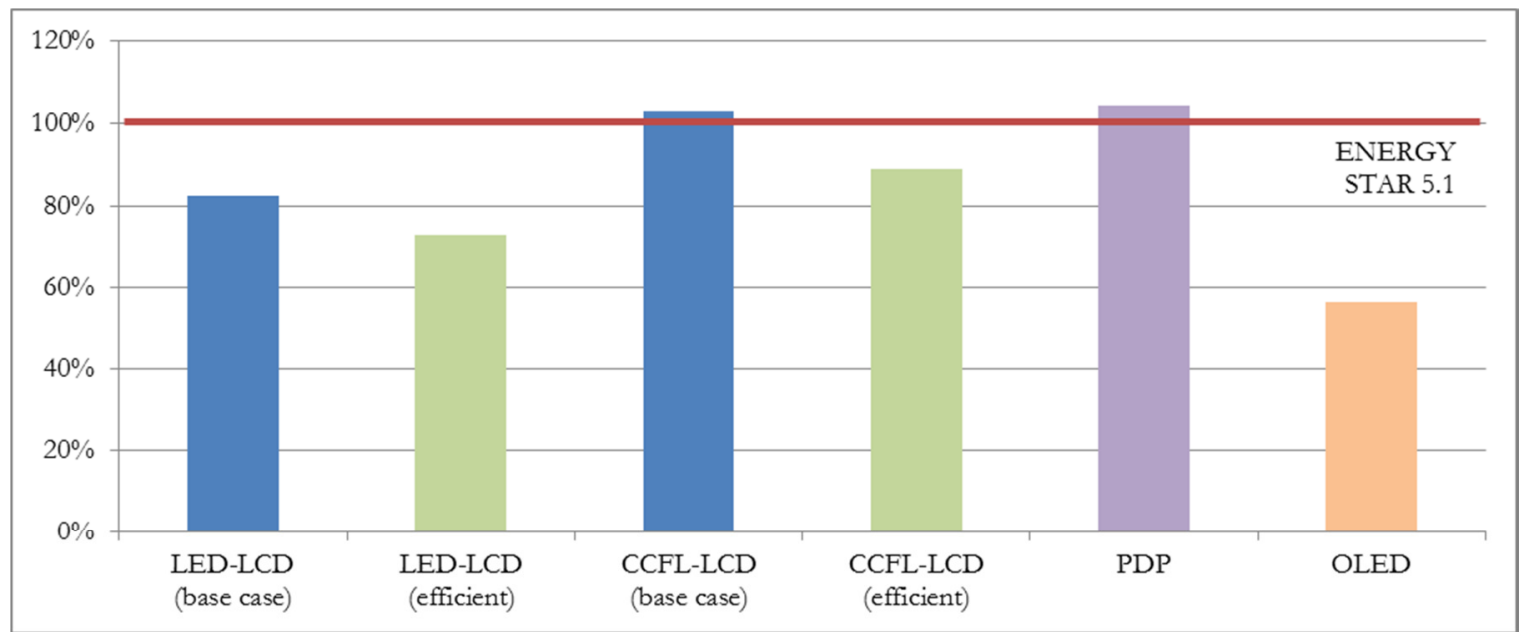

Figure ES-5 Estimated On-Mode Power for Display Technologies (2012, 42-inch TVs)

\section{C] Global Savings Potential}

Figure ES-6 shows a forecast of annual electricity savings potential in selected countries. It is important to note here that this savings potential reflects the difference between our assumed base case and efficiency case, and does not reflect additional savings from the current global level of electricity consumption due to the fast-moving and aggressive base case itself. Also, we assumed the efficiency case only for $30 \%$ of the LCD TV market. According to the forecast, the efficiency case is expected to further reduce energy consumption in LCD TVs by 156 TWh from 2012 through 2030. 


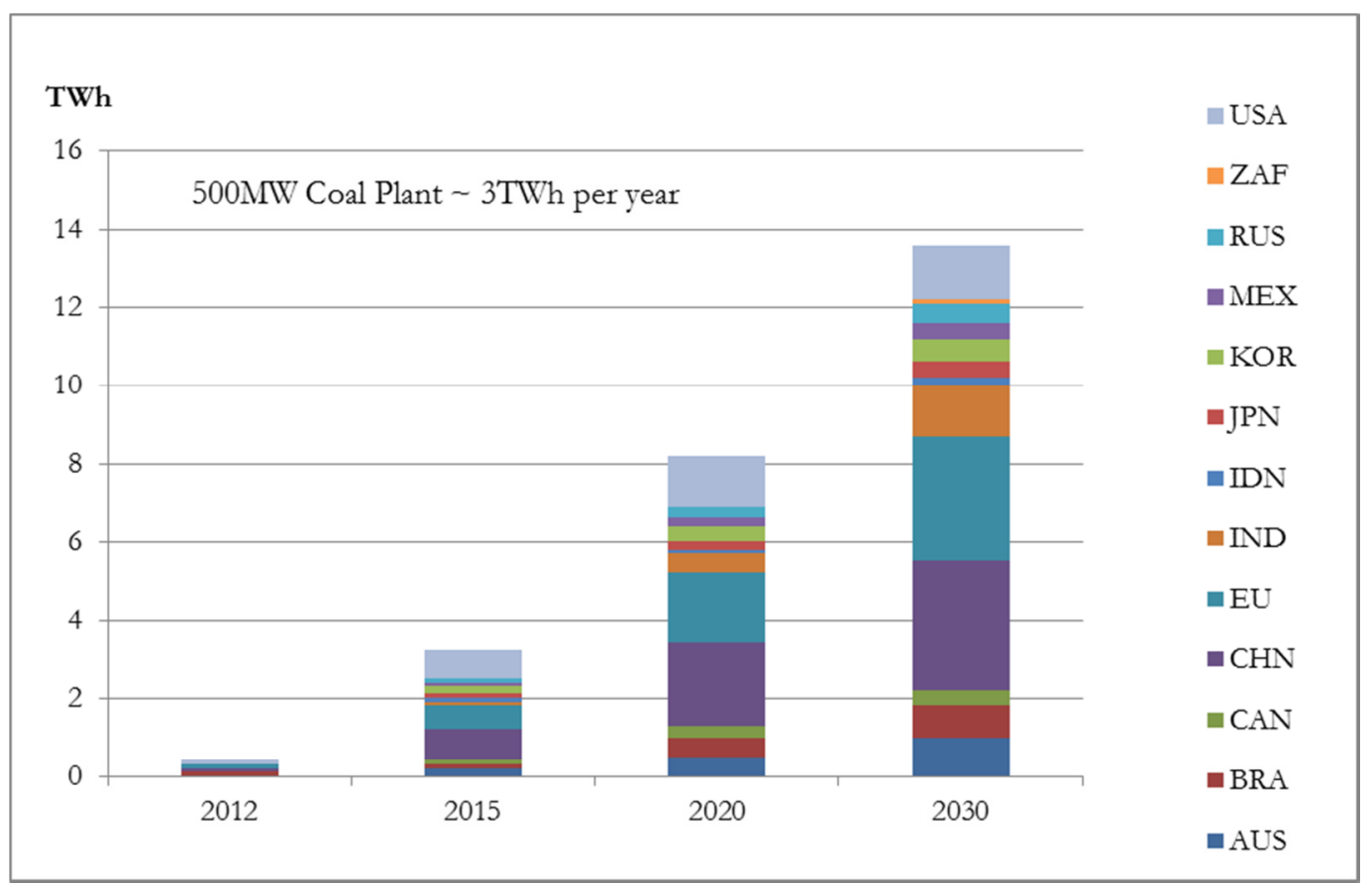

Source: Analysis using BUENAS model based on the methodology described in McNeil et al. (2008) and Chapter 5 of this report. $^{6}$

\section{Figure ES-6. Forecast of TV Electricity Savings Potential in Selected Countries}

\section{D] Key Lessons for Policy Makers}

\section{a. Impact of Increases in Screen Size/TV Sales on TV On-mode Power}

Although it is expected that both average TV screen size and TV sales will increase, the large-scale transition from CCFL-LCD TVs to LED-LCD TVs is expected to reduce the impact of countervailing trends in screen size and TV sales on total TV power consumption even without efficiency improvements. As a result, the addition of efficiency improvements will be sufficient to negate the impact of countervailing trends in screen size and TV sales on total TV power consumption. Figure ES-7 shows the average screen area per unit and global TV shipment by technology for 2010 and 2014. Figure ES-8 shows estimates of the total on-mode power consumed by all new TVs in 2014 for both the no-improvement case and the efficiency improvement base case.

\footnotetext{
${ }^{6}$ Our projections take into account the rapid improvements in TV efficiency resulting from a shift towards LCD TVs and from improvements in plasma TVs. We also take into account the rapid projected shift towards LCD TVs with LED backlighting.
} 

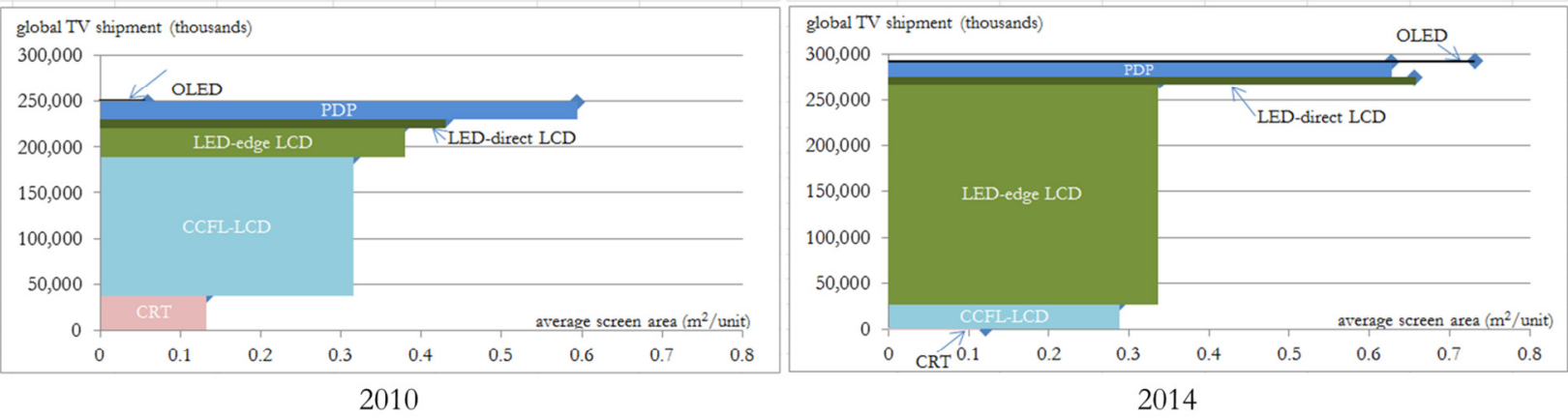

Source: Author's calculation based on DisplaySearch 2011f

Figure ES-7. Global TV Shipment and Average Screen Area by Technology in 2010 and 2014

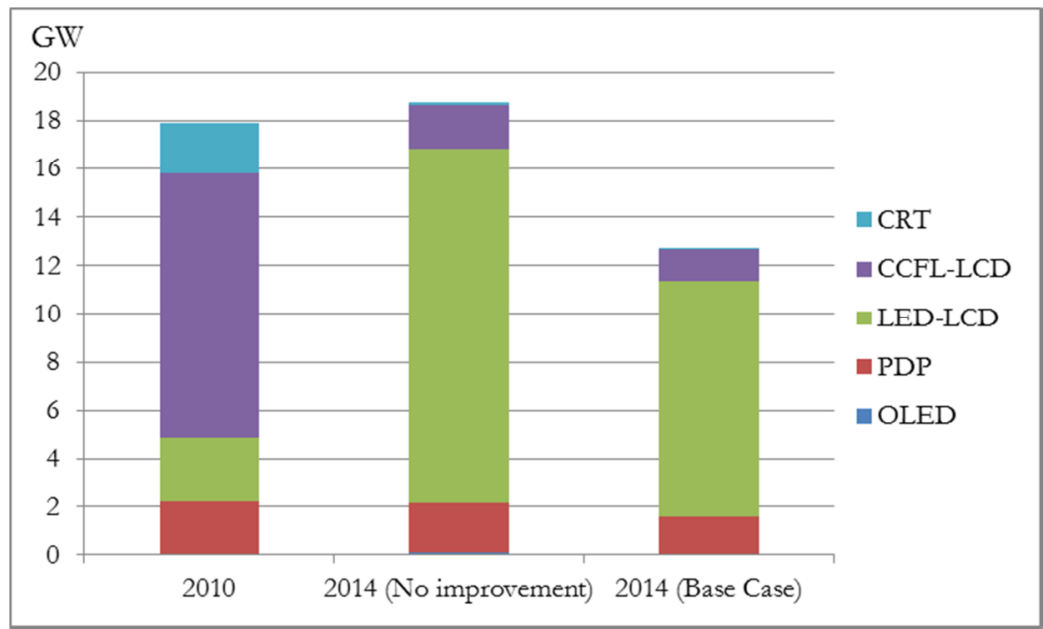

Figure ES-8. Estimates of Total On-mode Power Consumption by Global TV Shipment in 2014

\section{b. Stringency of Standards}

Standards and labeling programs need to take into account expected rapid improvements in TV efficiency and define much more stringent efficiency targets than are currently in place. For example, in 2008, market penetration of ENERGY STAR Version 3 qualified TVs was about 80\%; in 2010 that of ENERGY STAR Version 4 qualified TVs was 70\%; and in 2012, most TV models on the U.S. market $(>60 \%)^{7}$ will be able to meet ENERGY STAR Version 5 requirements. However, there are cost-effective options to further improve TV efficiency; if these options are adopted, then most TVs will perform better than ENERGY STAR Version 5, and minimum energy performance standards as stringent as ENERGY STAR Version 5 would be possible.

Key efficiency improvement options that could further improve TV efficiency are cost-effective (i.e., the Cost of Conserved Energy (CCE) < 8 cents/kilowatt hour [kWh]); thus, market transformation programs such as standards and incentives that will facilitate the adoption of these technologies are

\footnotetext{
7 We assume that most LED TVs will be able to meet ENERGY STAR Version 5.
} 
likely to be cost effective.

\section{c. Impact of Standards on Countervailing Screen Size/Energy Trends}

The average energy consumption of TVs is expected to increase with progressive increases in the sizes of TVs sold. Assuming that average TV on-mode power just meets the efficiency metric used for maximum power consumption in ENERGY STAR Version 4, and all other factors remain the same, average TV power is likely to increase by $9 \%$ with the expected $6.5 \%$ increase in TV size $(13.4 \%$ in screen area) between 2010 and 2014.

Taking into account the likely success of ENERGY STAR Version 5 in holding down energy consumption despite the expected increase in TV screen size, we assumed for our analysis that all TVs sold in North America from 2012 to 2014 qualify for ENERGY Version 5. Based on this assumption, we calculate that, in spite of expected increases in TV sales and screen sizes, annual TV energy consumption will be lower than the case in which TVs qualify only for ENERGY Version 4 from 2010 to 2014.

We note here that ENERGY STAR Version 5 will be functioning very much like a cap on on-mode power, controlling the power consumption of very large screen sizes. Similarly, the UK Energy Saving Trust recommended an endorsement scheme for digital TVs with a cap of $150 \mathrm{~W} .^{8}$

\begin{tabular}{l|r|r|r}
\hline & \multicolumn{1}{|c|}{2010} & \multicolumn{1}{|c}{2012} & \multicolumn{1}{c}{2014} \\
\hline Average Screen Size (North America) & 36.0 inch & 37.8 inch & 39.2 inch \\
\hline Average Screen Area (North America) & 553.4 inch $^{2}$ & 610.1 inch $^{2}$ & 656.1 inch $^{2}$ \\
\hline TV Shipments (million units) & 42.7 & 47.7 & 52.4 \\
\hline U.S. ENERGY STAR VERSION 4 & $\mathrm{P}_{\max }=91.4 \mathrm{~W}$ & - & - \\
\hline U.S. ENERGY STAR VERSION 5 & - & $\mathrm{P}_{\max }{ }^{*}=69.2 \mathrm{~W}$ & $\mathrm{P}_{\max } *=73.1 \mathrm{~W}$ \\
\hline
\end{tabular}

$\mathrm{P}_{\max }=0.120 \times \mathrm{A}+25,\left(\mathrm{~A}:\right.$ inch $\left.^{2}, \mathrm{~A} \geq 275\right)$

$P_{\max } *=0.084 \times A+18\left(A:\right.$ inch $\left.^{2} 1,068 \geq A \geq 275\right)$

Source for average screen size and TV shipments: DisplaySearch 2011a

Table ES-1. Increase in TV Screen Sizes and Shipments for North America

8 http://www.energysavingtrust.org.uk/business/content/view/full/481857 


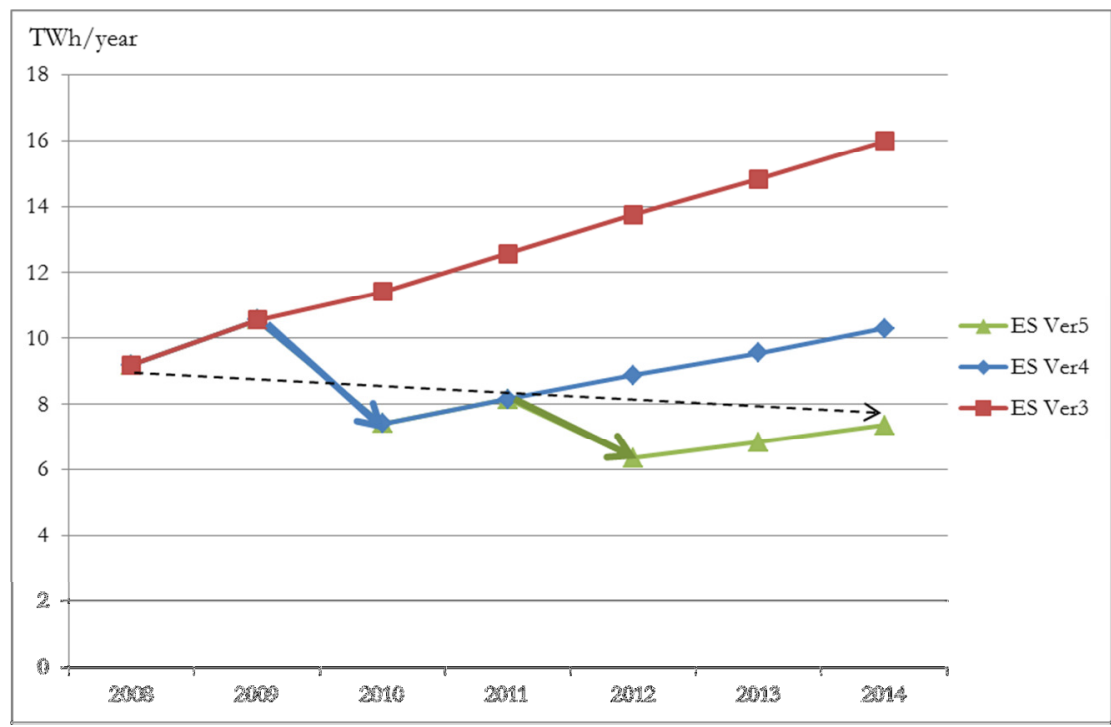

Figure ES-9. Effect of Predicted Screen Size and TV Shipments on Energy Consumption?

d. Options for Efficiency Improvement of Typical TVs also apply to 3D TVs and Smart TVs Although the trend toward incorporating a 3D function in TVs will result in increase in power consumption in 3D mode, all of the options for increasing the efficiency of 2D-only TVs will apply equally to $3 \mathrm{D}$ TVs. A TV in $3 \mathrm{D}$ mode requires about 3 to $5 \mathrm{~W}$ for $3 \mathrm{D}$ image processing at a minimum, and may consume more power, up to $20 \mathrm{~W}$ for 42-inch LCD and PDP TVs, depending on manufacturer settings. If we assume that all 3D TVs (LCDs and PDPs) consume an additional 20 W for 3D mode and 2 hours per day for 3D content, the additional cumulative energy consumption in 3D mode of the 3D TVs from 2010 to 2014 is estimated to be about 3 TWh. One issue in existing 3D TVs is that 3D mode causes $60-80 \%$ of light loss when compared to the luminance in $2 \mathrm{D}$ mode. This loss can therefore result in the brightness ${ }^{10}$ perceived by the viewer in $3 \mathrm{D}$ mode being lower than the brightness perceived in $2 \mathrm{D}$ mode, although $3 \mathrm{D}$ perception renders the image subjectively brighter than it technically is. It is expected that this issue will be overcome as both 3D technologies and display efficiency improve over time. Also, 3D content available to consumers is still limited, which makes it hard to project viewing hours for 3D content at this stage. Connected TVs (or Smart TVs) require advanced hardware components such as a central processing unit (CPU), graphical processing unit (GPU) and memory; the overall effect of these components on on-mode power is not significant, but local dimming methods will not be very useful in lowering power consumption for white internet screens. It is more important to look at effect of these new trends on overall energy consumption rather than their stand-alone effect on device power. Connected TVs have the potential to increase standby mode power because of network connectivity and fast start options. In addition, increased penetration of both 3D TVs and connected TVs may encourage consumers to buy larger screens and extend viewing hours. If both $3 \mathrm{D}$ TV and connected TV

\footnotetext{
9 Assuming that average daily viewing time is 5 hours and average standby power is $1 \mathrm{~W}$.

10 This refers to the brightness of the image perceived by the viewer in its final usable form due to losses in 3D glasses. The actual luminance of the screen would be the same or above for a constant effective brightness.
} 
functions are incorporated into entry-level TV models, the resulting increased energy consumption will to some degree offset the effect of efficiency improvements in TVs.

\section{e. Slow Boot Time May Prompt Consumers to Choose Fast Start Options which Significantly Increase Standby Power.}

One issue in currently available TVs is booting (or reactivating) time which consumes from a few seconds to about 45 seconds. Slow boot time can increasingly feature idle modes ("fast play", "quick start") that allow the TV system to power up within a few seconds from these modes. According to a recent report from ECCJ (ECCJ 2009), fast start options contribute about 25 W on average (Min 11.7 W, Max 68.0 W) to standby power consumption in a TV. Slow boot (or wake) time may prompt consumers to choose a fast start mode although the mode consumes significantly more power than the default standby.

\section{f. Rapidly Increasing Network Standby Power Indicates the Necessity for its Inclusion in} Standards and Test Procedures.

Although a majority of TVs consume less than $1 \mathrm{~W}$ in passive standby or sleep mode, newer connected TVs (or smart TVs) consume from $3 \mathrm{~W}$ to $30 \mathrm{~W}$ in network standby mode. The maximum power required to keep network connectivity is not expected to significantly increase, but the average network standby power is expected to increase, depending on the power management regime and reactivation time (i.e., resume-time-to-application). If we assume that all new connected TVs (or smart TVs) consume $30 \mathrm{~W}$ in network standby, the annual energy consumption in network standby for the new connected TVs in 2014 is about 27 TWh. There are many options to keep overall network standby power consumption low, but at a minimum including network standby power in the test procedures would be an important first step. Figure ES-10 shows a forecast for total energy consumption in network standby power from 2010 to 2014.

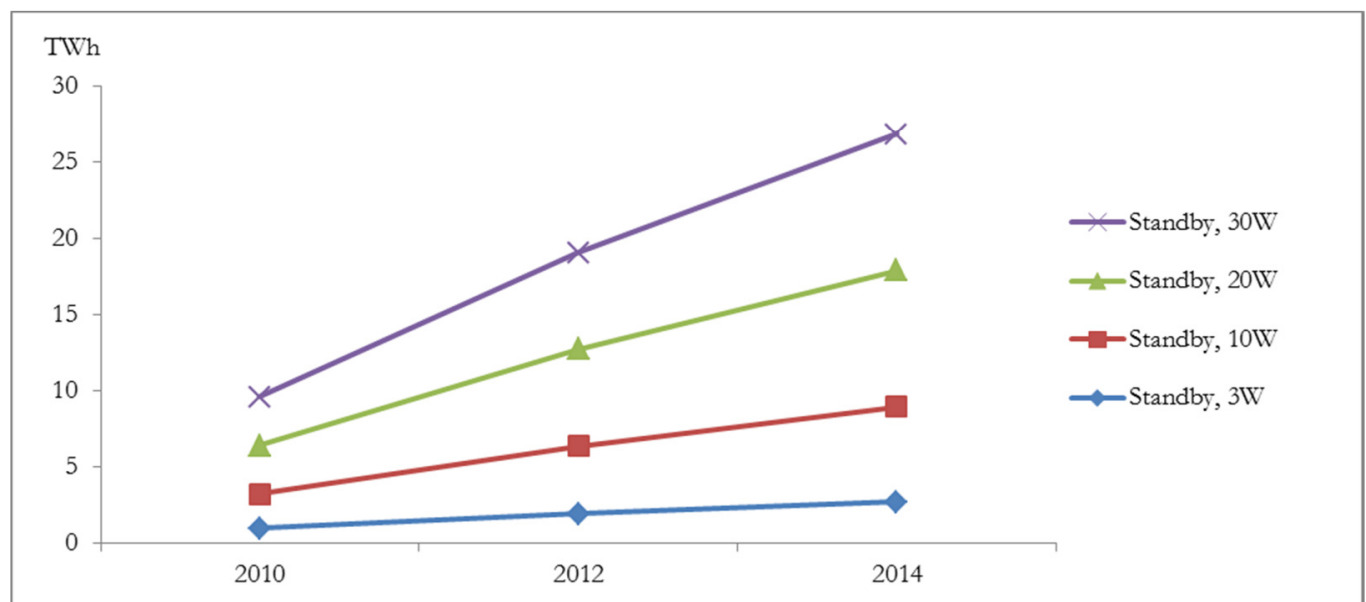

Figure ES-10. Estimates of Energy Consumption in Network Standby Mode by Global Connected TV Shipment Forecast

g. Effect of the Japanese Eco-Points Program on the Japanese TV Market

According to DisplaySearch (2011a), Japan experienced significant growth - more than 30\% - in the domestic TV market in 2009 and 2010 because of the government Eco-Points subsidy program, but 
a sharp decline is expected after the program ends in 2011. This example illustrates the sensitivity of consumer demand to financial incentive programs.

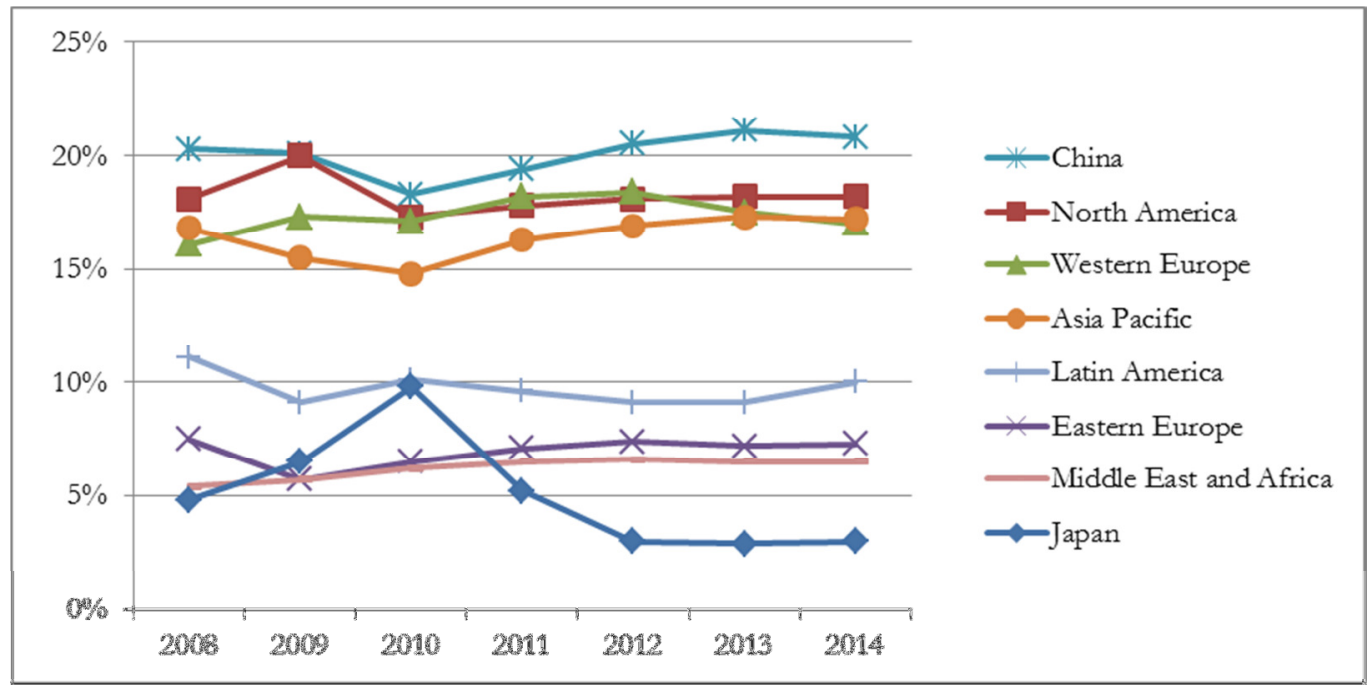

Source: DisplaySearch 2011a

Figure ES-11. Regional Distribution of Global TV Market

\section{h. Auto Brightness Control/Ambient Light Sensing as an Energy-Efficiency Option}

The magnitude of the effect of auto brightness control (ABC) on power consumption is significant. However, test procedures that allow for crediting estimated energy savings to TVs that have ABC can provide counterproductive incentives to manufacturers to set the $\mathrm{ABC}$ settings so that the picture is too dark for viewer comfort during testing in the dark. Such ABC settings would allow the manufacturer to claim energy savings during testing, but the user would then be prompted to disable the $\mathrm{ABC}$ for a brighter screen, which would negate any such savings in practice. All major manufacturers currently provide at least one model of TV with $\mathrm{ABC}$ activated. To ensure that $\mathrm{ABC}$ is properly used as an efficiency improvement option:

- ABC settings need to be activated by default for all TVs eligible to participate in market transformation programs.

- $\mathrm{ABC}$ settings need to be easier to adjust than to deactivate completely.

- The test method to account for $\mathrm{ABC}$ needs to be revised to test at more realistic ambient lighting levels.

The draft ENERGY STAR Version 6 (ENERGY STAR 2011c) includes an updated test method for ABC. On-mode power consumption for TVs with ABC is expected to be calculated as a weighted average of power consumption at various ambient lighting levels that are agreed upon by manufacturers.

\section{i. Effect of ABC on On-mode Power of ENERGY STAR-qualified TVS}

Most ENERGY STAR-qualified TVs over 40 inches have ABC settings enabled when shipped, which reduce the average on-mode power consumption for these models. Activating $A B C$ as the default setting for small- and medium-screen-size TVs could reduce on-mode power for those 
models as well. On average, ABC settings save up to 30\% of on-mode power although the savings vary among manufacturers and TV models. According to a data set from ICF International, which has provided technical and analytical support for the ENERGY STAR program, 17\% of ENERGY STAR-qualified LCD and PDP TV models do not meet ENERGY STAR Version 4 criteria with $\mathrm{ABC}$ disabled. In particular, it appears that shipping units with $\mathrm{ABC}$ enabled is an effective option to reduce on-mode power of CCFL-LCD TVs and PDP TVs. Figure ES-12 shows the different profile of on-mode power for ENERGY STAR-qualified TVs with ABC enabled and with ABC disabled.

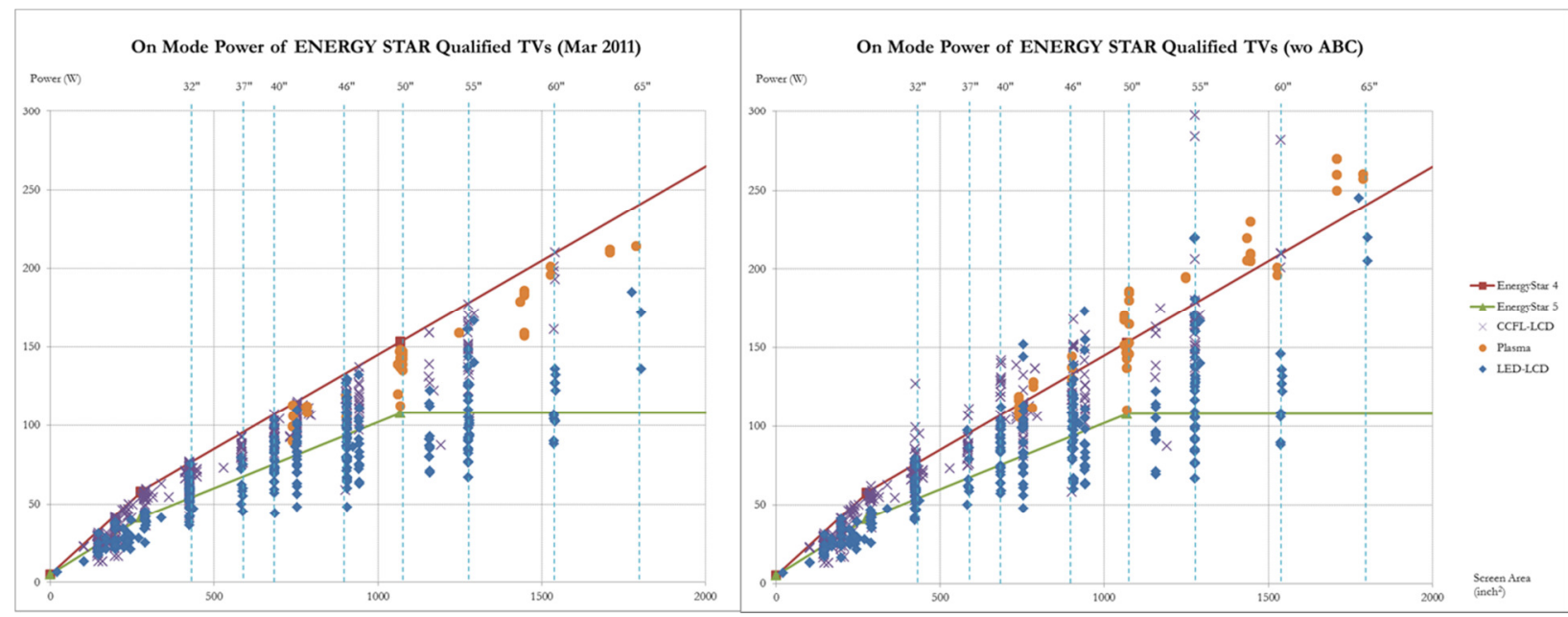

Figure ES-12. On Mode Power of ENERGY STAR TVs with ABC enabled and with ABC disabled

\section{j. Summary of Energy Consumption in TVs}

Based on the findings from the analysis, it is not expected that a significant increase in energy consumption in on-mode of global TV stocks will happen, because of the large-scale transition toward LED-LCD TVs and rapid efficiency improvement in TVs, in spite of the projected growth in screen size and TV sales. However, increasing features such as network connection and fast start mode is likely to increase energy consumption in standby mode of new TVs.

\begin{tabular}{l|l|l|r|r}
\hline \multicolumn{2}{c|}{ Modes } & $\begin{array}{l}\text { Contribution } \\
\text { from }\end{array}$ & \multicolumn{1}{c|}{2010} & \multicolumn{1}{c}{2014} \\
\hline \multirow{2}{*}{ On } & Normal & global stock & $168 \mathrm{TWh}$ & $171 \mathrm{TWh}$ \\
\cline { 2 - 5 } & 3D mode & global sales & $0.01-0.04 \mathrm{TWh}$ & $0.3-1.2 \mathrm{TWh}$ \\
\hline \multirow{3}{*}{ Standby } & Network Standby & global sales & $1.0-9.6 \mathrm{TWh}$ & $2.7-26.9 \mathrm{TWh}$ \\
\cline { 2 - 5 } & Fast Start Mode & global sales & $2.6-7.9 \mathrm{TWh}$ & $3.9-19.3 \mathrm{TWh}$ \\
\cline { 2 - 5 } & Data Acquisition & global sales & $0.2-0.7 \mathrm{TWh}$ & $0.3-1.0 \mathrm{TWh}$ \\
\hline
\end{tabular}

Table ES-2. Summary of Energy Consumption Forecast in TVs 


\section{Background, Scope, and Methods}

The U.S. Department of Energy (DOE) commissioned the International Energy Studies group at Lawrence Berkeley National Laboratory to undertake this technical analysis of television (TV) efficiency in support of the Super-efficient Equipment and Appliance Deployment (SEAD) initiative. The subsections below describe SEAD, the scope and data sources for this project, and the organization of the remainder of this report.

\subsection{Super-Efficient Equipment and Appliance Deployment Initiative (SEAD)}

The SEAD initiative aims to transform the global market by increasing the penetration of highly efficient equipment and appliances. SEAD is a government initiative whose activities and projects engage the private sector to realize the large global energy savings potential from improved appliance and equipment efficiency. SEAD seeks to enable high-level global action by informing the Clean Energy Ministerial dialogue as one of the initiatives in the Global Energy Efficiency Challenge. In keeping with its goal of achieving global energy savings through efficiency, SEAD was approved as a task within the International Partnership for Energy Efficiency Cooperation (IPEEC) in January 2010.

SEAD partners work together in voluntary activities to: (1) "raise the efficiency ceiling" by pulling superefficient appliances and equipment into the market through cooperation on measures like incentives, procurement, awards, and research and development (R\&D) investments; (2) "raise the efficiency floor" by working together to bolster national or regional policies like minimum efficiency standards; and (3) "strengthen the efficiency foundations" of programs by coordinating technical work to support these activities.

Although not all SEAD partners may decide to participate in every SEAD activity, SEAD partners have agreed to engage actively in their particular areas of interest through commitment of financing, staff, consultant experts, and other resources. In addition, all SEAD partners are committed to share information, e.g., on implementation schedules for and the technical detail of minimum efficiency standards and other efficiency programs. Information collected and created through SEAD activities will be shared among all SEAD partners and, to the extent appropriate, with the global public.

As of April 2011, the governments participating in SEAD are: Australia, Brazil, Canada, the European Commission, France, Germany, India, Japan, Korea, Mexico, Russia, South Africa, Sweden, the United Arab Emirates, the United Kingdom, and the United States. More information on SEAD is available from its website at http://www.superefficient.org/. 


\subsection{Scope and Methods}

The objective of this analysis is to identify potential TV efficiency improvements and their incremental costs, as well as to provide initial global and country-specific estimates of total energy savings potential. The overarching goal is to provide relevant and appropriate information to support design of appropriate policy programs that will accelerate the penetration of super-efficient TVs.

\section{Efficiency Improvements Analyzed}

This report defines three categories of potential TV efficiency improvement: market, economic, and technical. The analysis addresses market and economic efficiency improvements that are technically feasible, practical to manufacture, and therefore could be realized in the short term. In addition, we discuss significant technology trends to provide a picture of the future TV market and analyze technical improvements that are feasible in the short term, but we do not analyze in detail long-term technical efficiency improvements that would require R\&D investment. Our analysis compares future TV energy consumption for two scenarios: a base case, which assumes options that are common in manufacturers' roadmaps are implemented for all types of TV models, and an efficiency case, which assumes that all cost-effective efficiency options are implemented for targeted TVs, e.g., entry-level models in 32- to 42-inch liquid crystal display (LCD) TVs. LCD TVs in the 32- to 42 -inch size range are expected to account for about $60 \%$ of the global TV market in 2014, and, according to an expert, approximately $60 \%$ to $70 \%$ of models in that size range are estimated to be entrylevel models. While the cost-effective options to improve efficiency identified in this report apply regardless of screen size, some TV models at the higher end of the large screen sizes already employ these options.

\section{Data Sources}

The analysis team obtained the data for this report from the following sources: review of literature including technical reports; country-specific databases (i.e., U.S. ENERGY STAR and Energy Conservation Center, Japan); international conferences and exhibitions; and interviews with manufacturers and experts in the field. The experts and manufacturers interviewed have 7 to 15 years' experience in the TV industry, and their collective expertise covers the entire TV market, including cathode ray tube (CRT)/LCD/plasma display panel (PDP)/organic light emitting diode (OLED) technologies, R\&D planning, and TV testing. The identities of the expert and manufacturer sources are kept confidential at the interviewees' request because of business competitiveness concerns. Therefore, when information from these sources appears in the report text, it is attributed simply to "manufacturers" or "experts."

\section{Energy Savings Modeling}

Energy savings modeling in this report is supported by the Bottom-Up Energy Analysis System (BUENAS), which is an end-use energy forecasting model developed by Lawrence Berkeley National Laboratory (LBNL) and supported by the Collaborative Labeling and Appliance Standards Program (CLASP). BUENAS is a "bottom up" model that calculates energy demand for appliance types based on input data for individual product types. The model uses a basic activity/intensity approach, first calculating the quantity of a given appliance type in each country in each year (Module 1) and then multiplying by unit energy consumption in each scenario (Module 2). A third module calculates the impact of efficiency programs on the national stock of appliances by tracking sales and retirements. Figure 1-1 shows the structure of the BUENAS analysis. Chapter 5, Estimates of Energy Savings Potentials, gives further detail. 


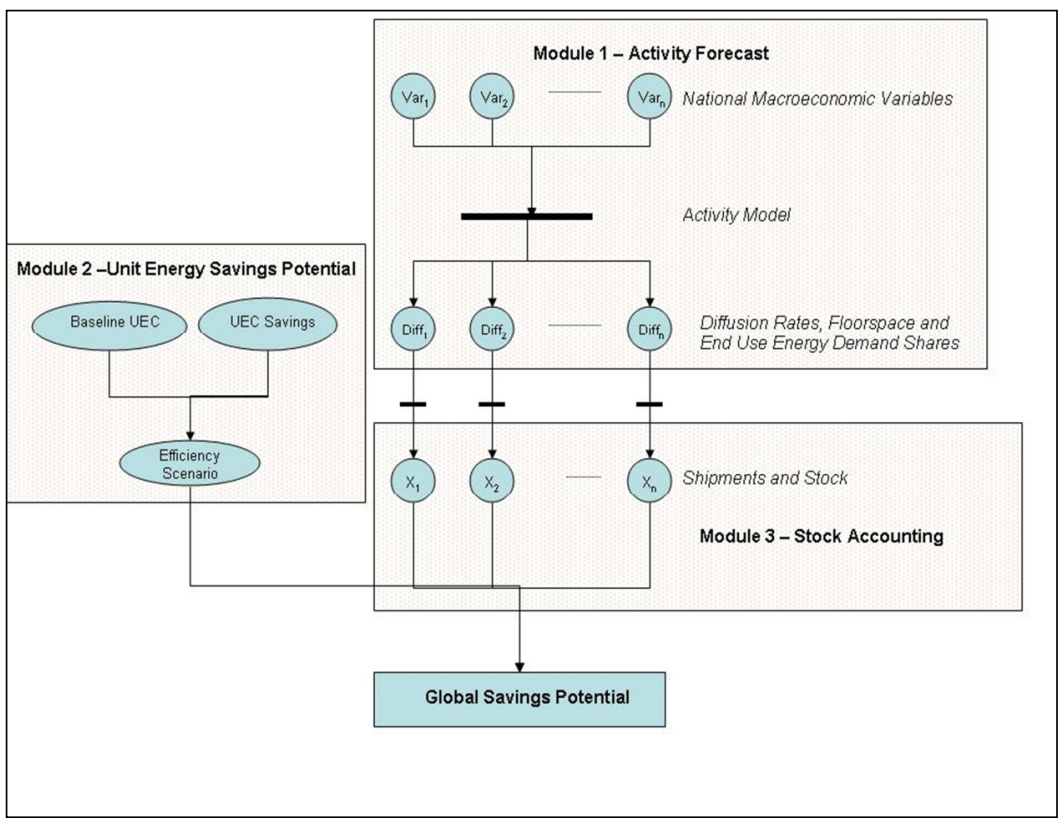

Source: McNeil et al. 2008

\section{Figure 1-1. BUENAS Analysis Structure}

\section{Analysis Method and BUENAS Data Inputs}

This report provided data inputs relevant to efficiency scenario and country-specific market forecasts for BUENAS Modules 1 and 2. The report's data analysis is based on global/regional TV market data, efficiency improvement options collected from various sources, and TV on-mode power data from ENERGY STAR and the Energy Conservation Center, Japan (ECCJ). Figure 1-2 shows the structure of data analysis used in this report.

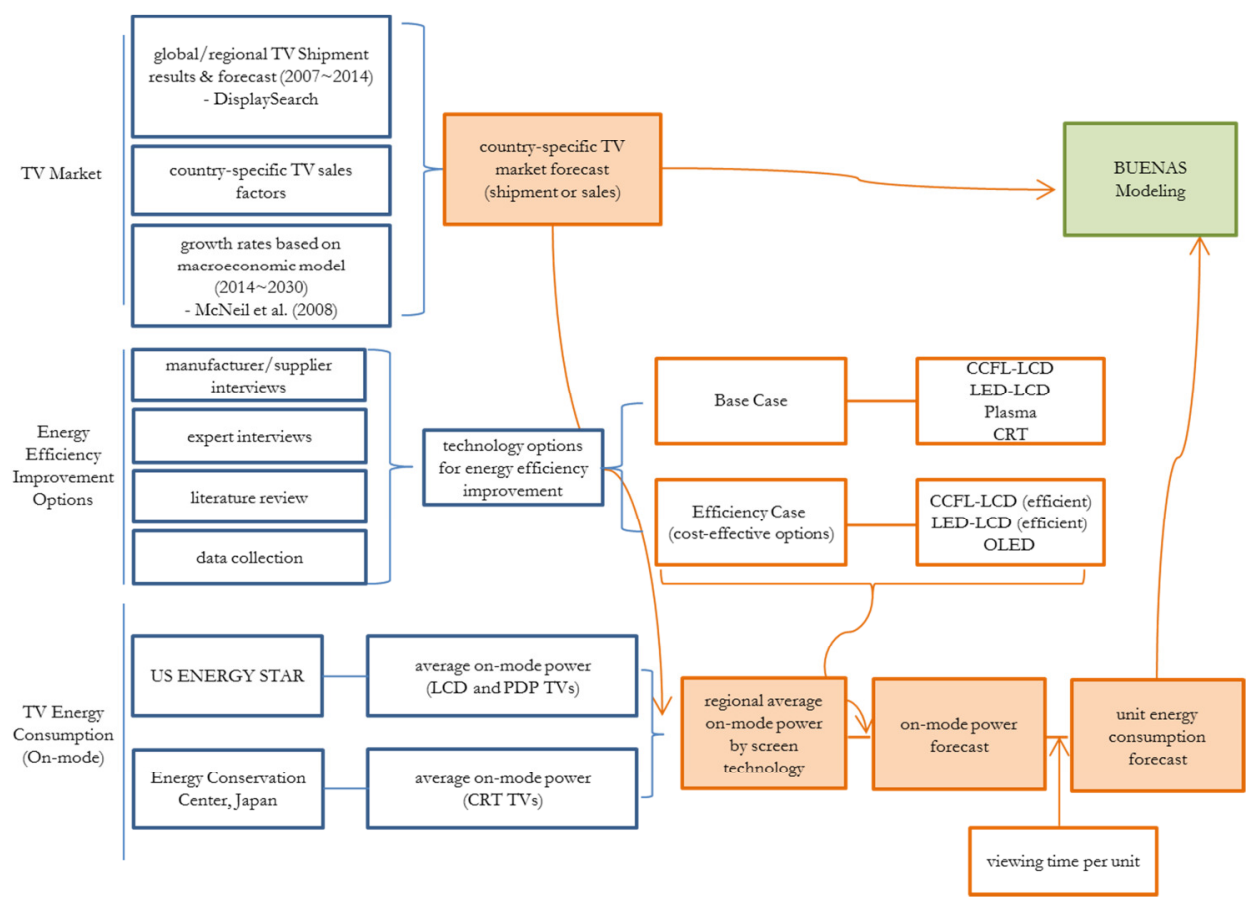

Figure 1-2. Data Analysis Structure 
The TV market data are based on DisplaySearch Quarterly Global TV Shipment and Forecast Reports (Q1 2010-Q1 2011). The reports provide TV market data according to screen technology, region, screen size, brand, and LCD backlight type. The forecast extends to the year 2014, and regional data are based on a survey of eight regions: Asia Pacific (AP), China, Eastern Europe (EE), Japan, Latin America (LA), Middle East \& Africa (ME\&A), North America (NA), and Western Europe (WE). Data are not provided on a country-specific basis except for China and Japan. Data for India are provided separately by DisplaySearch. The authors of this report were not able to refer directly to other countries' TV market data, so some simplifying assumptions were used to estimate TV shipments (or sales) for SEAD-1411. Data for Brazil, China, Japan, and India were taken directly from DisplaySearch. In accordance with recommendations from DisplaySearch, we assumed that an estimated $89 \%$ of LCD TVs shipped to North America go to the U.S., and Canada makes up the remaining $11 \%$. For other countries, country-specific factors are assumed from discussion with manufacturers and experts. Those factors are based on recent LCD TV sales in SEAD-14. The country-specific factors are applied uniformly to all TV product categories: CRT, LCD, PDP, and OLED. Each country's TV shipment growth rate from 2010 to 2014 is the same as that of corresponding region defined by DisplaySearch. Details for the TV market are discussed in Chapter 2, TV Market Assessment.

The TV on-mode power data used in the analysis are from the ENERGY STAR TV qualified product list posted on www.energystar.gov November 1, 2010 (ENERGY STAR 2010) and March 22, 2011 (ENERGY STAR 2011). On-mode power data from ENERGY STAR qualified products may be less than TV power data in other regions, although these data include hundreds of TV models and represent a majority of the U.S. TV market. This analysis also refers to another TV power data set provided by ECCJ. Although these data do not include on-mode power values measured by the IEC 62087 standard, they do include rated power of the products listed, including CRT TVs. All of our on-mode power analysis for PDP and LCD TVs is based on the ENERGY STAR list, and additionally this report analyzes a relationship between rated power and onmode power from the Japanese data set. Appendix B, Data, Methods, and Assumptions, presents details of the data analysis.

\subsubsection{Efficiency Improvement Potentials}

\section{Market Potential (included in base case)}

Sathaye and Phadke (2010) ${ }^{12}$ defined market potential in a manner that, if applied to the current analysis, would refer to the changes in energy efficiency that might be expected to occur under predicted market conditions. Our market potential analysis is based on broad technology trends in and recent manufacturer roadmaps of efficiency improvements. These improvements are typically a result of market demand, strategic product development, and expected future trends in efficiency standards and labeling programs. We include in the category of market potential those technical options or trends that are expected to save energy without any additional policy intervention. For the purposes of this analysis, all such options are included in the base case.

\section{Economic Potential (included in efficiency case)}

Energy efficiency is limited by factors (often referred to as market failures) that relate to markets, public policies, and other influences that inhibit the diffusion of technologies that are (or are projected to be) costeffective for users. Amelioration of this class of market imperfections would increase efficiency to a level that Sathaye and Phadke (2010) label economic potential. Economic potential represents the level of energy efficiency that could be achieved if all efficiency improvement options were implemented that are cost effective from the consumer point of view. Although our economic potential analysis discusses some options for efficiency improvement that require additional R\&D investment, it focuses primarily on options that

\footnotetext{
11 This report is analyzing only those countries that were members of SEAD as of CEM-1.

12 Sathaye and Phadke (2010) define market and economic potential for greenhouse gas mitigation technologies. We adapted these definitions for our study of TV efficiency technologies.
} 
require removal of market imperfections rather than long-term R\&D investment.

\section{Technical Potential (discussed briefly, out of the scope of this analysis)}

Even if all market, institutional, social, and cultural barriers whose removal is cost effective from a societal perspective were eliminated, some technologies might not be widely used simply because they would still be too expensive. Technical potential is the maximum technologically feasible extent to which efficiency could be improved through technology diffusion. Our study does not address these options in depth because it is intended to provide timely support for SEAD-14's decision making. It therefore focuses on technical options that are expected to be commercialized in the short term and does not attempt an exhaustive analysis of the long-term technical potential for energy savings in TVs. However, we discuss select options that fall into the category of technical potential and that require R\&D investment, to provide context where appropriate.

\subsubsection{Technology 'Trends}

Our analysis of recent TV technology trends focuses on projecting the impacts of these trends on TV energy consumption. Since roughly the mid-2000s, the TV industry has been experiencing a significant market transition from traditional CRT TVs to flat panel display (FPD) TVs, including LCD and PDP TVs. The adoption of FPD TVs has mainly been driven by advanced picture quality, growing screen size, and innovative manufacturing processes; however, new technical challenges are related not only to improvements in display technology. They also include overarching lifestyle trends that could redefine the product itself, for example three-dimensional (3D) TVs and smart TVs (or connected TVs). These innovations add new roles and functionalities to existing screen technologies such as LCDs, PDPs, and OLEDs. These trends and their relative magnitude within the overall TV market will have considerable impact on total energy consumption from TVs. Both of these trends are discussed in detail in Chapter 3, TV Technology Trends and Energy Consumption.

\subsubsection{Factors Affecting TV Power Consumption}

In general, TV on-mode power consumption correlates with display screen area; additional minimum power consumption is attributable to non-display components such as the digital signal processing unit, audio, interface, and power supply unit (PSU) (Fraunhofer 2007f). Because the minimum power consumption varies with features or performance other than screen size, this component needs to be considered separately. Onmode power consumption can also be controlled by ambient light sensing, depending on various external light conditions, and by dimming (or auto-power control), depending on signal images. This report uses a simplified formula in calculating on-mode power. Table 1-1 shows key factors affecting TV on-mode power.

$P_{T V \text { on }}=($ Screen Area $) \times P_{\text {screen }}+P_{\text {basic }} \ldots \ldots(1)^{13}$

\footnotetext{
13 This simplified formula might not be appropriate to deal with TVs with additional integrated functionality. For other functionality such as video storage and digital tuners, additional terms need to be included, i.e., $P_{\text {TV on }}=($ Screen Area $) \times P_{\text {screen }}+P_{\text {basic }}+$ $P_{\text {feature }}$. (Fraunhofer 2007f) However, according to IEC $62087 \mathrm{Ed} \mathrm{2,} \mathrm{the} \mathrm{functionalities} \mathrm{included} \mathrm{in} P_{\text {feature }}$ are supposed to be deactivated during the $\mathrm{ON}$-mode power measurement process.
} 


\begin{tabular}{l|l}
\hline \multicolumn{1}{c|}{ Technical factors that affect $P_{\text {display }}$} & Technical factors that affect $P_{\text {basic }}$ \\
\hline - Panel technologies (LCD, PDP, OLED, etc.) & \\
- Panel efficiency (cell design, materials, driving scheme, etc.) & $\cdot$ Resolution \\
- Backlight unit efficiency (light source, films, etc.) & - Frame Rate \\
- Screen size & - Digital signal process \\
- Efficiency of power supply unit & $\cdot$ Efficiency of power supply unit \\
- Resolution & \\
\hline
\end{tabular}

Table 1-1. Key Factors Affecting TV On-Mode Power

\section{A] Reference Power: $P_{\text {basic }}$}

Reference power is not directly affected by screen size but is mainly determined by image signal process. According to manufacturers, reference power in a normal TV set is between 18 watts (W) and $25 \mathrm{~W}$ at onmode power measurement. Table 1-2 shows technical factors that affect TV reference power.

\begin{tabular}{l|l}
\hline Technical factors that increase $P_{\text {basic }}$ & Technical factors that decrease $P_{\text {basic }}$ \\
\hline - High resolution & \\
- High frame rate & $\cdot$ Development of system large-scale integration $(\mathrm{LSI})^{14}$ \\
- 3D image process & $(100$ nanometers $[\mathrm{nm}]>65 \mathrm{~nm}>45 \mathrm{~nm}>30 \mathrm{~nm})$ \\
$\cdot$ Advanced image algorithm & $\cdot$ Power reduction algorithm \\
- Multi-purpose screen (game, internet) & \\
\hline
\end{tabular}

\section{Table 1-2. Technical Factors that affect TV Reference Power Consumption}

This report assumes that the average reference power consumption ( $\mathrm{P}_{\text {basic }}$ ) is $20 \mathrm{~W}$ for TVs analyzed for the year 2010 even though $\mathrm{P}_{\text {basic }}$ varies with TV specification. ${ }^{15}$ This is because 1) the individual effect of each factor on reference power is not fully assessed here as a result of limited data, and 2) it is difficult to break down market share of TVs according to additional functionality. This report assumes that technology improvements can reduce $\mathrm{P}_{\text {basic }}$ over time.

The technology options discussed this analysis are focused on power consumption of displays ( $\left.\mathrm{P}_{\text {display }}\right)$ excluding non-display components, as described by equation (2) below, rather than that of the TV sets as described in equation (1) above. Therefore, it is necessary to convert display power consumption improvement potentials to equivalent TV power consumption improvement potentials.

$P_{\text {display }}=($ Screen Area $) \times P_{\text {screen }} \ldots$.... (2)

where $\mathrm{P}_{\text {screen }}$ is expressed in $\mathrm{W}$ per screen area.

\footnotetext{
${ }^{14}$ Large Scale Integration means the process of manufacturing integrated circuits by integrating thousands of transistors into a single chip. "XX nm (nanometers) process" indicates the technology generation; smaller numbers are more advanced.

15 U.S. EPA ENERGY STAR Version 4 requirements specify the following values for $\mathrm{P}_{\text {basic }}$ from the $\mathrm{P}_{\max }$ equation: $5 \mathrm{~W}$ for TVs with screen area less than 275 square inches (equivalent to 1,774 square centimeters), and $25 \mathrm{~W}$ for TVs with screen area equal to or greater than 275 square inches. In the European Union labeling program's Energy Efficiency Index (EEI), Pasic is as follows: 20 W for TV sets with one tuner/receiver and no hard disc; $24 \mathrm{~W}$ for TV sets with hard disc(s); $24 \mathrm{~W}$ for TV sets with two or more tuners/receivers; $28 \mathrm{~W}$ for TV sets with hard disc(s) and two or more tuners/receivers; and $15 \mathrm{~W}$ for TV monitors.
} 
B] Unit Power: $P_{\text {screen }}\left(\frac{\text { Watts }}{\text { screen area }}\right)$

\section{Screen Technologies: LCD, PDP, and OLED}

This report focuses on technology options and efficiency improvement potentials for screen technologies. Because market forecasts indicate that LCD and PDP TVs will account for more than $90 \%$ of the global TV market from the year 2011 forward, our analysis focuses on these two categories of products. In addition to LCDs and PDPs, we also discuss OLED displays as an emerging technology. In terms of screen size associated with improvement options, the analysis focuses on 32- to 42-inch $(81.3-\mathrm{cm})$ light-emitting diode (LED) backlit LCD TVs, 42- to 50-inch (106.7- to 127.0-cm) PDP TVs, and 30- to 42-inch (76.2- to 101.6-cm) OLED TVs because of their existing or expected market significance. Details are discussed in Chapter 4, TV Efficiency Improvement Potentials. This report does not discuss Surface Emissive Display (SED) technology because the authors did not see any positive sign from the industry about technological progress or market availability of SED in the short term.

\section{Power Management}

There are two types of on-mode power management technologies for TV screens. One is Auto Brightness Control (ABC), which detects ambient light and dims the screen in low light conditions. More than $60 \%$ of ENERGY STAR-qualified TVs (ENERGY STAR 2011) have this function enabled by default when shipped. On-mode power of TVs with $A B C$ is weighted by the effect of $A B C$. This analysis discusses $A B C$ in Chapter 6, Further Discussion, but does not address technological details and their costs. The other on-mode-power management technology is backlight dimming of LCD TVs. While power consumption of PDPs and OLEDs varies with luminance of signal images, conventional LCD backlights are capable of "complete dimming" or "line dimming," and recent LED backlighting takes advantage of "local dimming" of LED lamps, depending on image sources. This report discusses dimming technology and corresponding cost for LED backlight units (BLUs) in Chapter 4, TV Efficiency Improvement Potentials.

\section{C] Screen Area}

Average screen area is expected to increase while $\mathrm{P}_{\text {screen }}\left(\mathrm{W} / \mathrm{cm}^{2}\right.$ or $\left.\mathrm{W} / \mathrm{in}^{2}\right)$ will be improved by various technology options. The average screen size (diagonally measured) is expected to increase from 32.4 inches $(82.0 \mathrm{~cm})$ in 2010 to 34.7 inches $(87.4 \mathrm{~cm})$ for all regions in 2014 . However, average screen size is also expected to saturate at some level after the year 2014. Technology options discussed in this analysis are related to unit power consumption (W/screen area) for the main screen sizes for each screen technology. Figure 1-3 shows data from the recent past as well as a forecast of total display area (in thousand square meters) for TV shipments of new TVs. The figure also shows average TV screen size (in inches). 


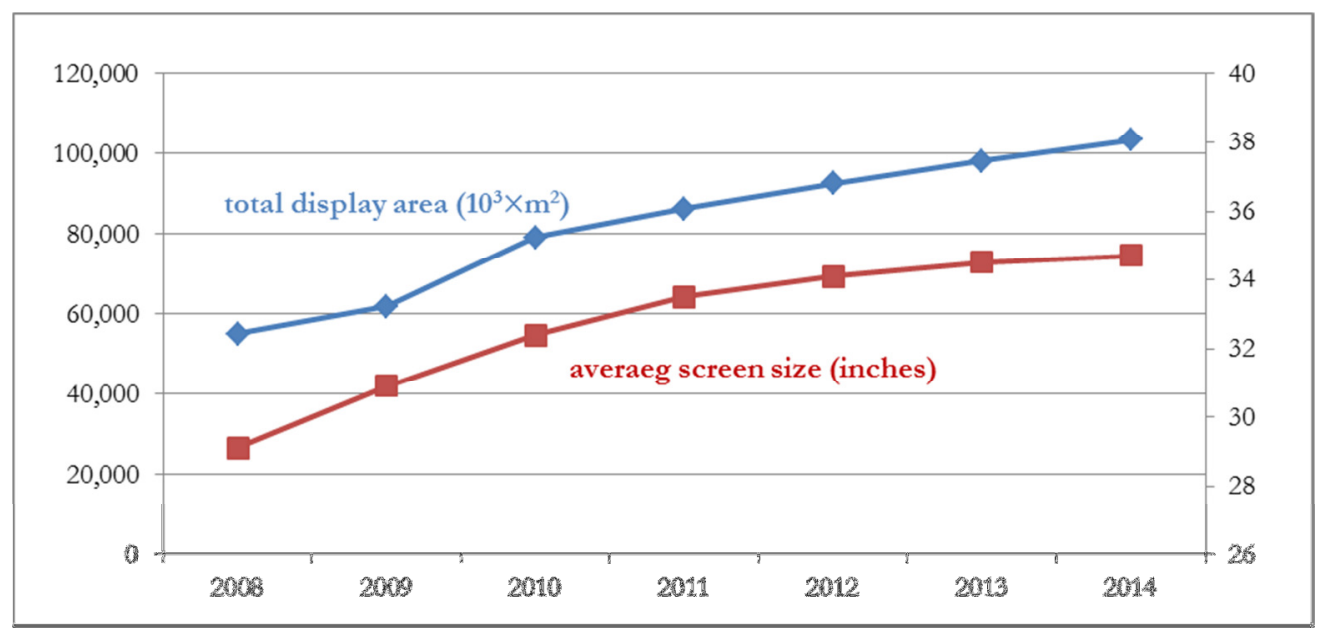

Source: DisplaySearch 2011f

Figure 1-3. Global Average TV Screen Size (diagonal) and Total Screen Area Forecast

Standby power, viewing hours, and TV sales affect total TV energy consumption. Viewing hours may be affected by technology trends, e.g., integration of internet/gaming functions into TVs, and consumer lifestyle changes. Annual TV sales will be affected by many variables including a region's economic status, market trends, and relevant policy instruments.

\section{D] Standby Power $\left(P_{\text {standby }}\right)$}

Most recently available TVs consume less than $1 \mathrm{~W}$ in passive standby mode; therefore, this analysis does not address specific technology options for standby power, and standby power is not included in estimates of potential energy savings. However, the recent growth of smart TVs (or connected TVs) may drive up network standby features and "quick start", in which screen and circuits are kept ready to be turned on within a couple of seconds. According to the EuP Preparatory Study (Fraunhofer 2010b), fast start options consume over $25 \mathrm{~W}$ in "hot" standby for complex TVs. This issue may become more important as connected TVs become more popular and may contribute significantly to TV energy use. This report discusses standby power and energy use in connected TVs in Chapter 6, Further Discussion.

\section{E] Average Viewing Time}

Average viewing time and number of TVs per household are major factors affecting total energy consumption. Although further research is necessary to fully analyze these trends, we tried to identify recent country-specific data for average TV viewing time. According to the International Energy Agency (IEA) 4E Mapping and Benchmarking report (IEA 2010a), viewing hours per unit vary with countries; the figures for the UK (4.8h), Australia (7.3h), and the Republic of Korea (6.9h) were based on government assumptions. The EuP Preparatory Study (Fraunhofer 2007e), which includes many studies that provide data on TV watching time, concluded that average viewing hours, i.e., daily on-time duration of the primary TV in a European household, is 4 hours per day. With growing functionality this average viewing time may increase in the future. According to the Organization for Economic Cooperation and Development (OECD 2009), average "household TV viewing time" for many countries had been almost constant under 4 hours over the prior 10 years, except in the U.S. The average American household's daily viewing hours were 7.7 in 2001 and 8.2 in 2007. The main reason for the difference between the U.S. and other countries could be related to the average number of TV sets per household. ${ }^{16}$ That is, one possible explanation is that the OECD survey did not take into account multiple TVs running in a household. It is important to know that different

16 The average number of TV sets per household in 2009 for the U.S. was estimated at 2.86 (IEA 2009). 
terminology, e.g., on-time, viewing time, and use time, may produce different results in surveys. Although average viewing time per TV is a major factor affecting total energy consumption, robust country-specific data for average TV viewing time per unit vary among studies. Therefore, further research is necessary to fully analyze these trends. Our analysis considers average daily viewing (on-mode) time per unit and regional TV shipments or sales. Assumptions for average viewing time are included in Appendix B, Data, Methods and Assumptions.

\subsection{Organization of this Report}

The remainder of this report is organized as follows:

Chapter 2, TV Market Assessment, discusses TV market trends by technology, region, and screen size.

Chapter 3, TV Technology Trends and Energy Consumption, discusses the impact of two major TV technology introductions, 3D TVs and internet-enabled TVs, on energy consumption.

Chapter 4, TV Efficiency Improvement Potentials, discusses technologically feasible energy-efficiency improvements that reduce TV energy consumption.

Chapter 5, Estimates of Energy Savings Potentials, presents TV energy savings potentials from the Bottom-Up Energy Analysis System (BUENAS).

Chapter 6, Other Issues Related to Power Consumption and Efficiency, discusses additional factors and issues that affect TV energy-efficiency improvements but are not covered in previous chapters.

Chapter 7, Summary and Conclusions, summarizes the previous chapters and offers conclusions and suggestions for further research.

Several appendices provide supporting information for this analysis as follows:

Appendix $\boldsymbol{A}$ lists SEAD participating countries and regional TV markets corresponding to those countries.

Appendix $\boldsymbol{B}$ describes data sources, methods, and the data process for the BUENAS model.

Appendix $\boldsymbol{C}$ presents a sensitivity analysis of the energy-efficiency improvement options.

Appendix $\boldsymbol{D}$ presents a cost of conserved energy analysis of the cost effective options discussed in the report.

Appendix $\boldsymbol{E}$ discusses the distinction between brightness and luminance. 


\section{TV Market Assessment}

This chapter discusses market trends by technology, region, and screen size based on the TV market forecast by DisplaySearch.

\subsection{TV Market Forecast by Technology}

Global TV shipments were 248 million units in 2010 and are expected to reach 288 million units in 2014, according to DisplaySearch (2011a, 2011f). Penetration of FPD TVs, such as LCD and PDP TVs, has increased significantly in recent years. LCD TVs are expected to dominate across the globe, growing from 190 million units in 2010 to 269 million units in 2014. PDP TVs are expected to remain viable because they have 3D technology advantages over LCD TVs. ${ }^{17}$ However, in the long run, the sales volume and market share of PDPs are expected to slowly decline as LCD TV 3D performance and production costs improve. OLED TVs with screen sizes over 30 inches $(76.2 \mathrm{~cm})$ are expected to be commercialized in 2012 but are not expected to be cost competitive against LCD TVs in the short term. The market share of CRT TVs will decrease at an accelerating rate (See Figure 2-1) as major CRT TV manufacturers stop mass production in the next 2 years. Although CRT TVs are expected to remain popular in some emerging markets for cost reasons, major TV brands are likely to provide more affordable LCD TVs to replace CRT TVs in these markets. Figure 2-1 shows global TV shipment and screen size forecast by technology.

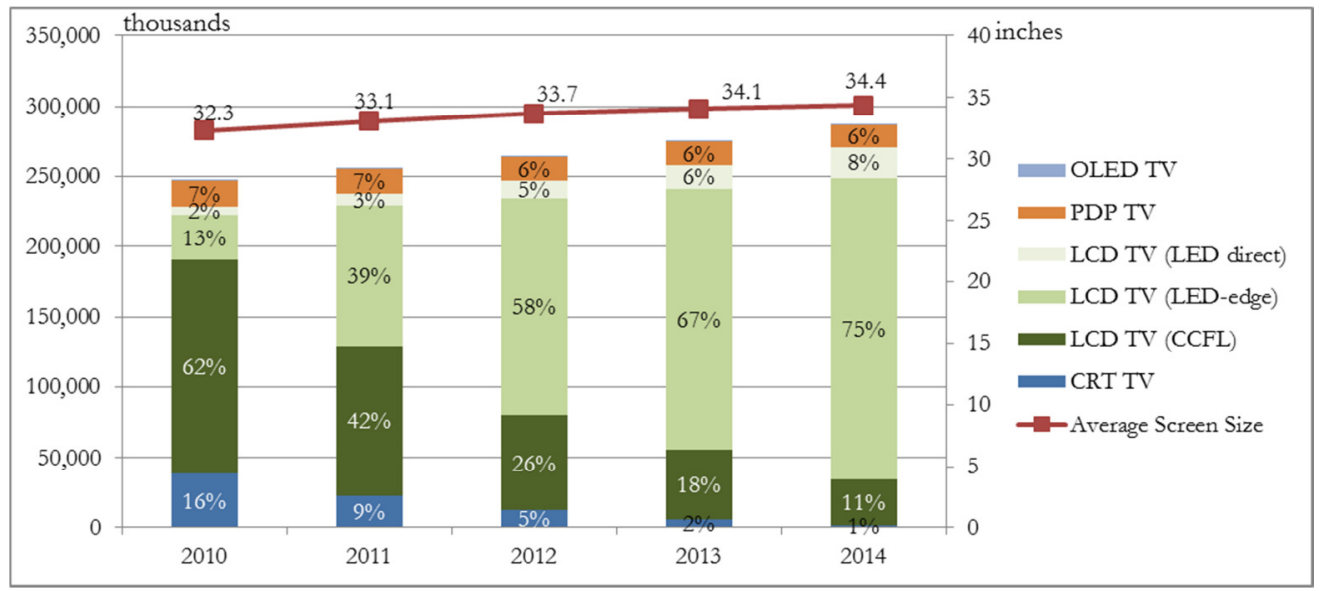

Source: DisplaySearch 2011a

Figure 2-1. Global TV Shipment and Screen Size Forecast by Technology

\subsubsection{LCD T'V Market Forecast by Backlight Type}

According to DisplaySearch (2011a), a large-scale transition from cold cathode fluorescent lamp (CCFL)LCD TVs to LED backlit LCD TVs is expected to happen between 2010 and 2014. Also, it is expected that there will be more demands for FPD TVs in developing countries to replace traditional CRT TVs as analog broadcast phases out. The rapid improvement in LED technologies has driven the adoption of LED backlights for TV application, which has been regarded as one of the best candidates to meet new energy efficiency standards for TVs. In line with expected increasing demand and rapid technological improvement, the production efficiency, or costs, will be rapidly improved as the number of TVs being produced increases. Supply side factors such as relatively high selling prices, better margins, and reduced logistics costs associated with thinner and lighter form factors are also contributing to the market transition. Among LCD TVs, LED-

17 PDPs, as an emissive type of display, have been said to provide more natural images than LCDs in moving scenes, and the incremental cost of 3D TVs is not significant compared to the cost of LCDs because current LCDs are required to employ high refresh rate, e.g., $200 / 240 \mathrm{~Hz}$, for 3D TV applications. 
edge backlit TVs are expected to be the mainstream technology particularly for screen sizes between 20 inches and 40 inches because their manufacturing costs are lower than those of LED-direct backlit TVs. LED-edge backlit TVs also have a better aesthetic profile, i.e., a slimmer body. Figure 2-2 shows the shift in relative percentages of LED-edge backlit TVs from 2010 to 2014. Some large screen sizes are still expected to use LED-direct backlight for high-end products because these devices can employ local dimming technology that can independently control each LED lamp, resulting in higher contrast ratio and better picture quality. We discuss this technical difference further in Chapter 3, TV Technology Trends and Energy Consumption.

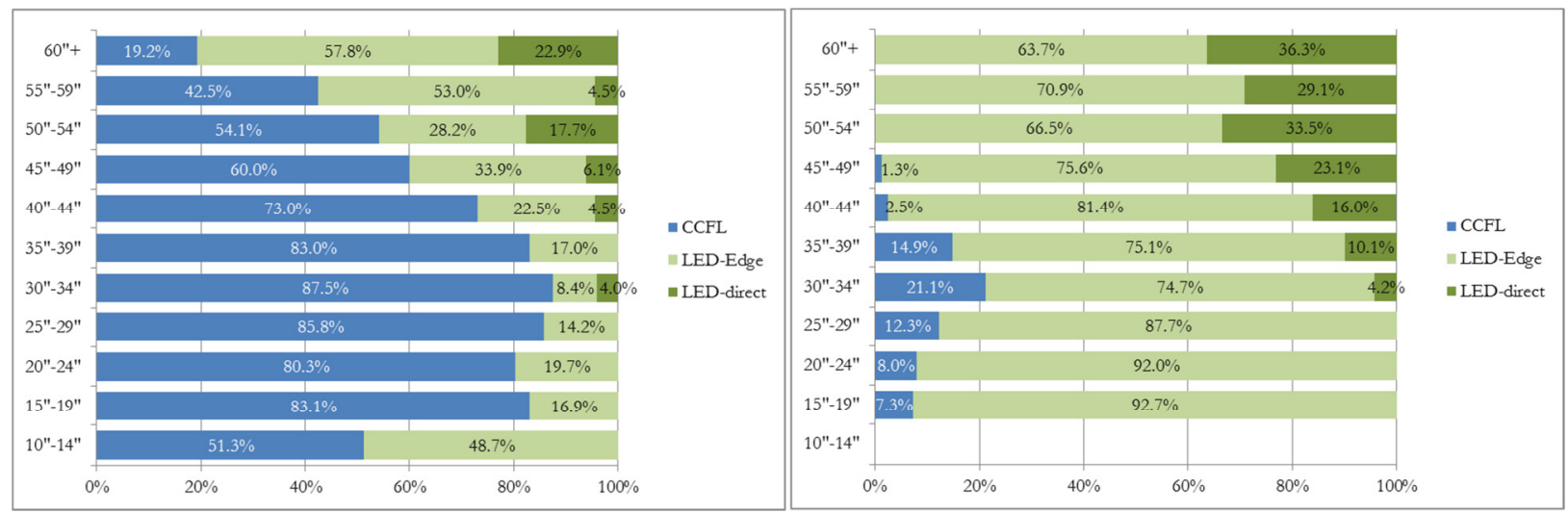

Source: DisplaySearch 2011a

Figure 2-2. LCD Backlight Migration by Screen Size

\subsubsection{PDP TV Market Forecast}

There has been the market difference between PDP and LCD technologies in relation to screen size. PDP market is almost entirely composed of large screen size over 40 inches. PDP TVs are expected to remain in 6 to $7 \%$ shares of global TVs. However, in the long run, the sales volume and market share of PDPs are expected to slowly decline as LCD TV production costs improve. Figure 2-3 shows that LCD share in screen size over 50 inches is expected to become larger than PDP share in 2014.

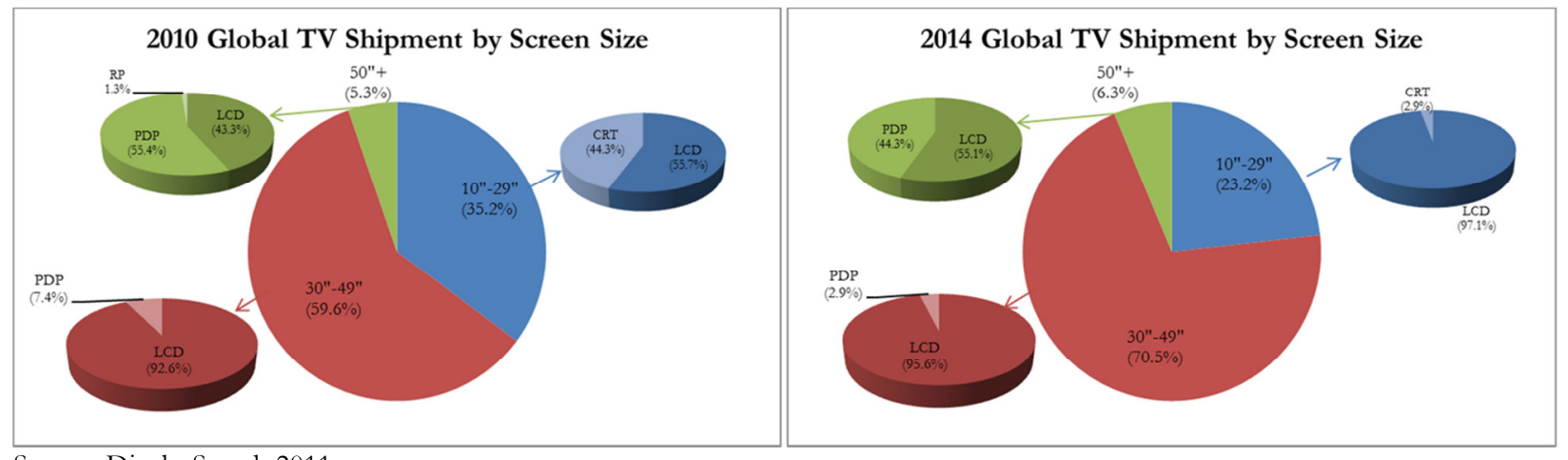

Source: DisplaySearch 2011a

Figure 2-3. Global TV Shipment by Screen Size

\subsubsection{OLED 'TV Market Forecast}

The number of OLED TVs ${ }^{18}$ has been growing rapidly in small-sized mobile applications, and major TV brands are adopting OLEDs for such applications Sony introduced an 11-inch (27.9-cm) OLED TV model in 2007, and LG Electronics launched a 15-inch (38.1-cm) OLED TV model in 2010. In addition, the following

18 OLED TVs in this report specifically refer to active matrix (AM) OLEDs. AM displays can independently control each pixel, while passive matrix displays use an X and Y axis grid to operate a pixel. All LCDs and OLEDs used in TVs are based on AM structure. 
OLED TV prototypes have been demonstrated at recent exhibitions: Sony's 27-inch (68.6-cm), Samsung's 30inch $(76.2-\mathrm{cm})$ and 40-inch (101.6-cm), and LG's 31-inch (78.7-cm). OLED TVs larger than 30 inches $(76.2$ $\mathrm{cm}$ ) are expected to be available in 2012. However, according to DisplaySearch (2011a), OLED TVs will not become cost competitive with LCD TVs in the short term, and global shipments will barely reach 1 million units $(0.4 \%)$. In a recent report (DisplaySearch 2011f), DisplaySearch expected that 40- to 55-inch (101.6 to $139.7 \mathrm{~cm}$ ) OLED TVs will be realized in the market, rather than the 30- to 39-inch $(76.2-$ to $99.1-\mathrm{cm})$ size band, because of low cost-competitiveness. At the early stage, it is likely that manufacturers will provide OLED TVs in 40 to 55 inches as flagship models. Table 2-1 shows the OLED TV market forecast. This forecast is uncertain because of the relative immaturity of OLED technology.

\begin{tabular}{l|r|r|r|r|r|r}
\hline Year & 2009 & 2010 & 2011 & 2012 & 2013 & 2014 \\
\hline Worldwide & 1.9 & 0.6 & 1.2 & 56.0 & 360.0 & $1,080.0$ \\
\hline
\end{tabular}

Source: DisplaySearch 2011a, unit: thousands

\section{Table 2-1. Global OLED TV Market Forecast}

According DisplaySearch (2011g), the average market price of 40-inch (101.6-cm) OLED TVs is expected to be about $\$ 1,800$ in the $1^{\text {st }}$ quarter of 2014 while the market price of comparable 40-inch (101.6-cm) LED backlit LCD TVs is expected to be less than $\$ 500$. Most consumers will not purchase OLED TVs until these devices become price competitive against LCD TVs, which is expected to take more than 3 years. Consequently, although we discuss OLED TVs as a technology of interest from an energy-efficiency perspective, we focus primarily on improvement of LCD TVs through 2014. Figure 2-4 shows the average forecast market price for 40-inch (101.6-cm) OLED TVs.

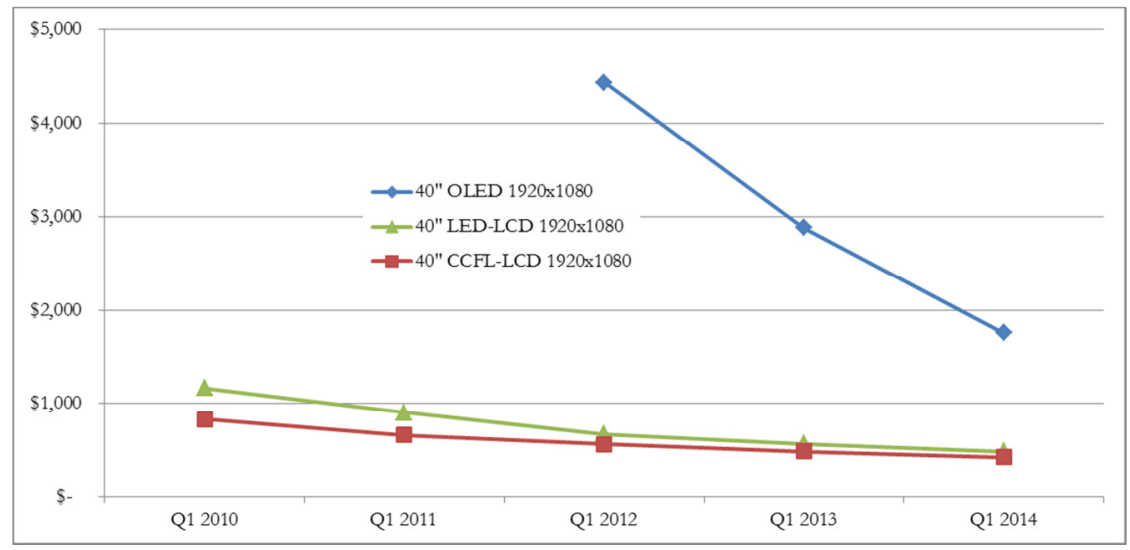

Source: DisplaySearch 2011g

Figure 2-4. Average Forecast Market Price for 40-inch OLED TVs ${ }^{19}$

\subsection{TV Market Forecast by Region}

DisplaySearch provides data for eight regional TV markets: Asia Pacific (AP), China, Japan, Eastern Europe (EE), Western Europe (WE), Latin America (LA), North America (NA), and the Middle East \& Africa (ME\&A). Countries included in these regions are listed in Appendix A. NA, WE, and China are currently the largest TV markets, and demand in the AP region is expected to keep growing as demand for LCD TVs increases and LCD TVs replace conventional CRT TVs. According to DisplaySearch (2011a), Japan's TV market grew more than 30\% in 2009 and 2010 because of the government Eco-Points subsidy program, but a sharp decline is expected after the program ends in 2011. Figure 2-5 shows the regional distribution of the global TV market from 2009 to 2014.

19 The market price is based on the typical specification of TV models in the U.S. market. 


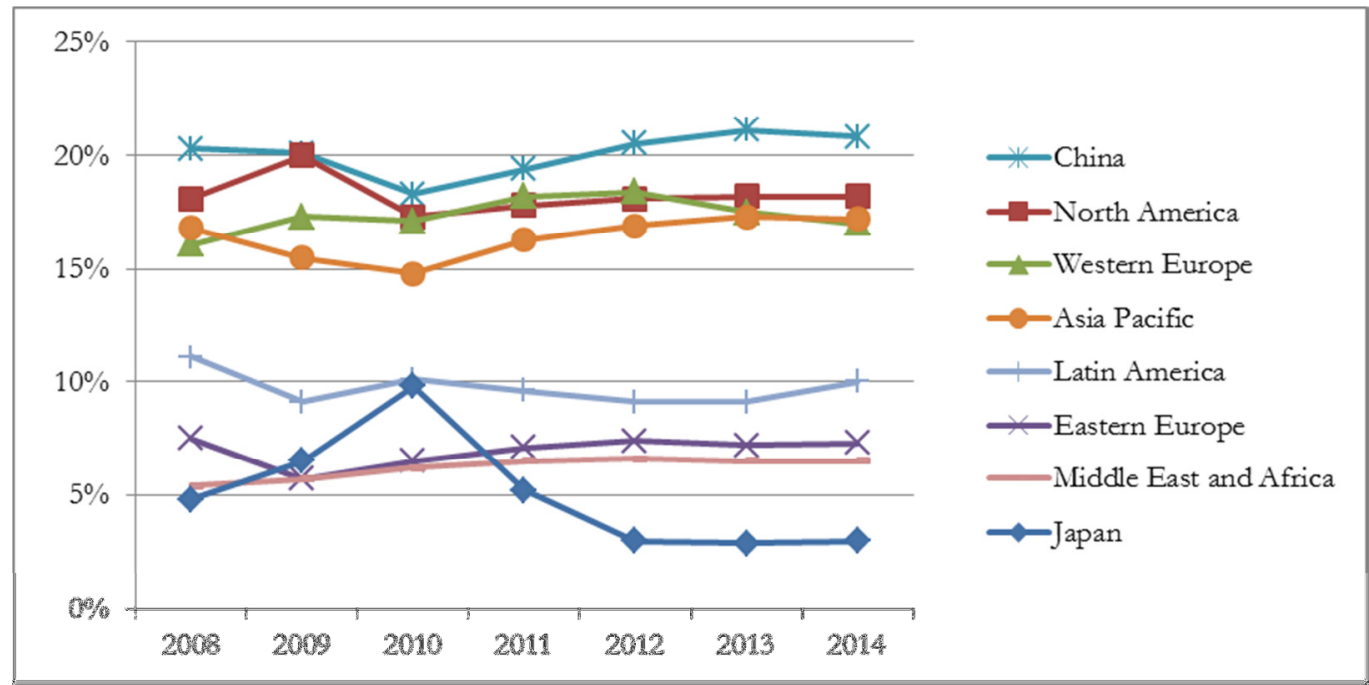

Source: DisplaySearch 2011a

Figure 2-5. Regional Distribution of Global TV Market from 2009 to 2014

CRT TVs still have significant market share in developing regions such as AP, LA, and ME\&A. However, the production of CRTs is expected to decline during the next few years, and the market price of LCD TVs is expected to become more affordable to consumers in those regions; as a result, CRTs are expected to be replaced by LCD TVs. Figure 2-6 shows the regional transitions in TV technologies from 2010 to 2014.

2010

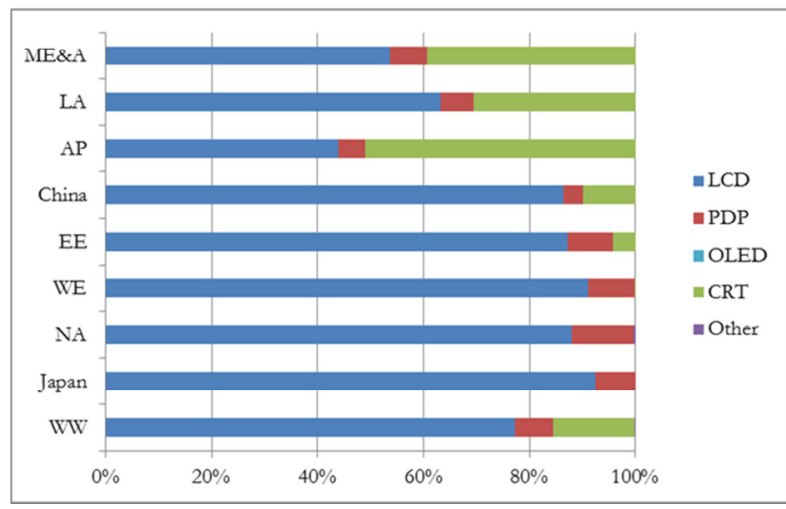

2014

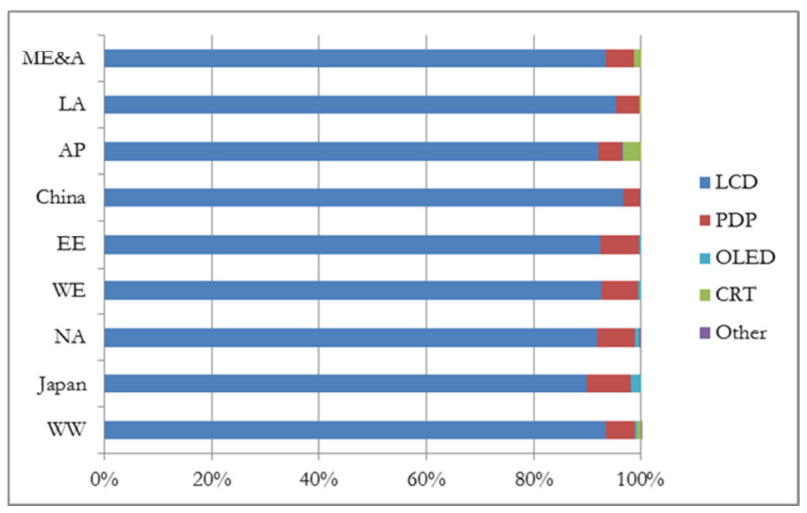

Source: DisplaySearch 2010c

\section{Figure 2-6. TV Technology Transition by Region}

For LCD TVs, LED-edge backlit TVs are expected to gradually replace CCFL backlit TVs in all regions during the next few years; however, CCFL backlit TVs may continue to have about $20 \%$ of the market share in developing regions because CCFL backlit TVs are more competitive against LEDs in terms of market price. Furthermore, consumers in developing regions replace their TVs less frequently than in other regions, adding to the lag in market adoption of LED-edge backlit TVs. Figure 2-7 shows the shift in relative numbers of CCFL, LED-edge, and LED-direct TVs from 2010 to 2014. 
CCFL $>>L E D$-edge $>\mathrm{LED}$-direct

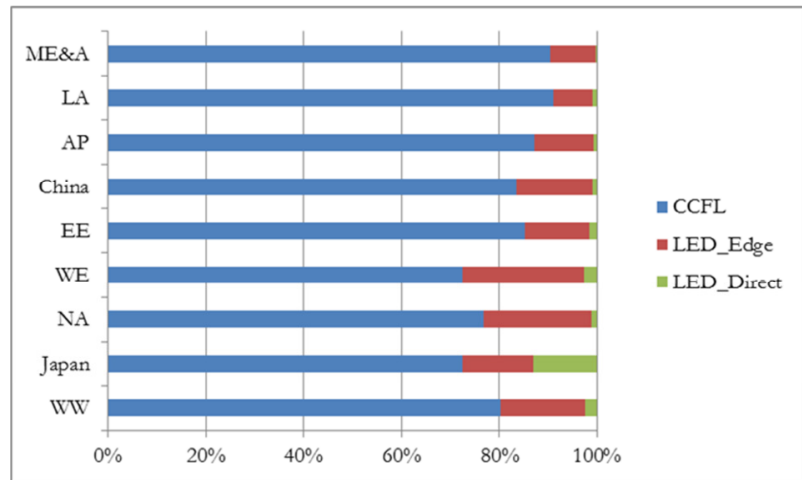

LED-edge $>$ LED-direct, CCFL.

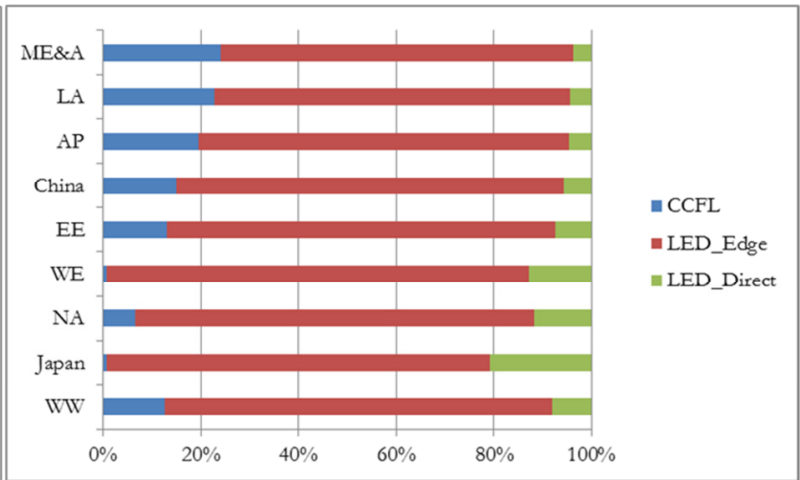

Source: DisplaySearch 2010c

Figure 2-7. LCD Backlight Migration by Region

In spite of the market perspective above for developing regions, manufacturers expect the trend toward increasing demand to accelerate as the average market price of FPD TVs continues to fall and FPD TVs become more affordable to more consumers. Figure 2-8 shows that average market prices of LCD TVs are expected to decrease by $30 \%$ to $40 \%$ from 2010 to 2014, and those of CRT TVs are expected to decrease by about $23 \%$. Although these average price trends represent the U.S. market, it is reasonable to assume that other markets will see a similar trend given the global concentration of TV manufacturing.

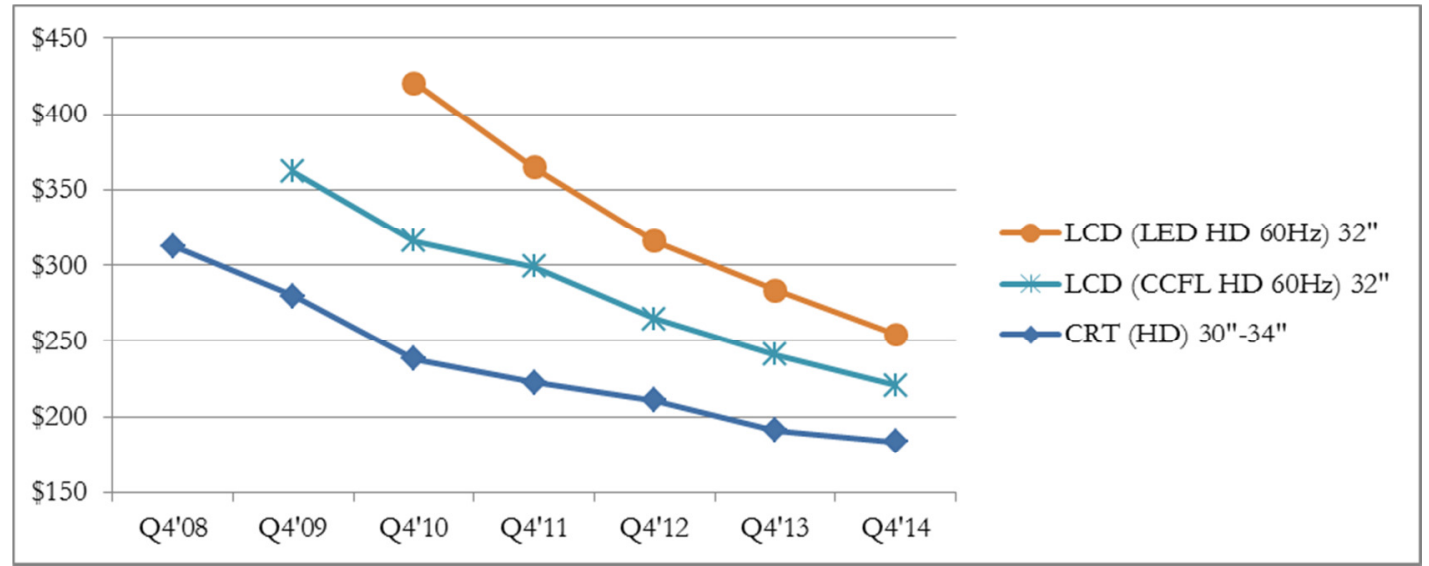

Source: DisplaySearch $2010 \mathrm{f}$

Figure 2-8. CRT and LCD TVs Market Price Forecast Based on U.S. models

Major TV brands are expected to provide more LED backlit TV models at lower prices in emerging markets. Manufacturers can accomplish this by decreasing the maximum luminance level and color-reproduction capability, which reduces material costs (DisplaySearch 2011b). The maximum luminance level of new entrylevel LED-LCD TVs in emerging markets is lower than that of typical TVs in mature markets by $23 \%$ to $33 \%$. This decreased luminance results from cost reduction measures, such as reducing the number of LEDs and using LEDs with low color-reproduction capability. These cost-reduction measures lower the maximum TV power consumption although precisely how they affect on-mode power consumption is unclear and needs further investigation. Lower luminance allows manufacturers to use fewer LED packages as well as low-voltage driven electronic parts in the circuitry. In addition, according to manufacturers, LEDs with low 
color-reproduction capability (i.e., color gamut ${ }^{20}$ ) are more efficient than high-color-gamut LEDs. Although many tradeoffs might be possible in the complex relationship among energy efficiency, cost, and picture quality, tradeoffs between picture quality and efficiency or picture quality and cost are not expected to become dominant in TVs for markets in developed regions where consumers are typically more conscious of quality than cost. Table 2-2 shows new design trends in entry-level LED-LCD TVs for emerging markets.

\begin{tabular}{l|r|c}
\hline \multicolumn{1}{c|}{ Typical Models } & Max. Luminance & Color Reproduction \\
\hline Mature Markets & $400-450 \mathrm{~cd} / \mathrm{m}^{2 *}$ & $>83 \%$ \\
\hline $\begin{array}{l}\text { Entry Models in } \\
\text { Emerging Markets }\end{array}$ & $300-360 \mathrm{~cd} / \mathrm{m}^{2}$ & $68-72 \%$ \\
\hline
\end{tabular}

$* \mathrm{~cd} / \mathrm{m}^{2}$ : candelas per square meter

Source: DisplaySearch 2011b

Table 2-2. New Design Trends in Entry-level LED-LCD TVs

As mentioned in Section 2.1.3., the market share of OLED TVs is expected to expand through 2014, although OLED TV market share would be below 1\% of the global TV market. In the short term, most OLED TVs will be available in developed regions such as NA, WE, and Japan because the average market price is expected to be very high. Figure 2-9 shows the OLED TV shipment forecast.

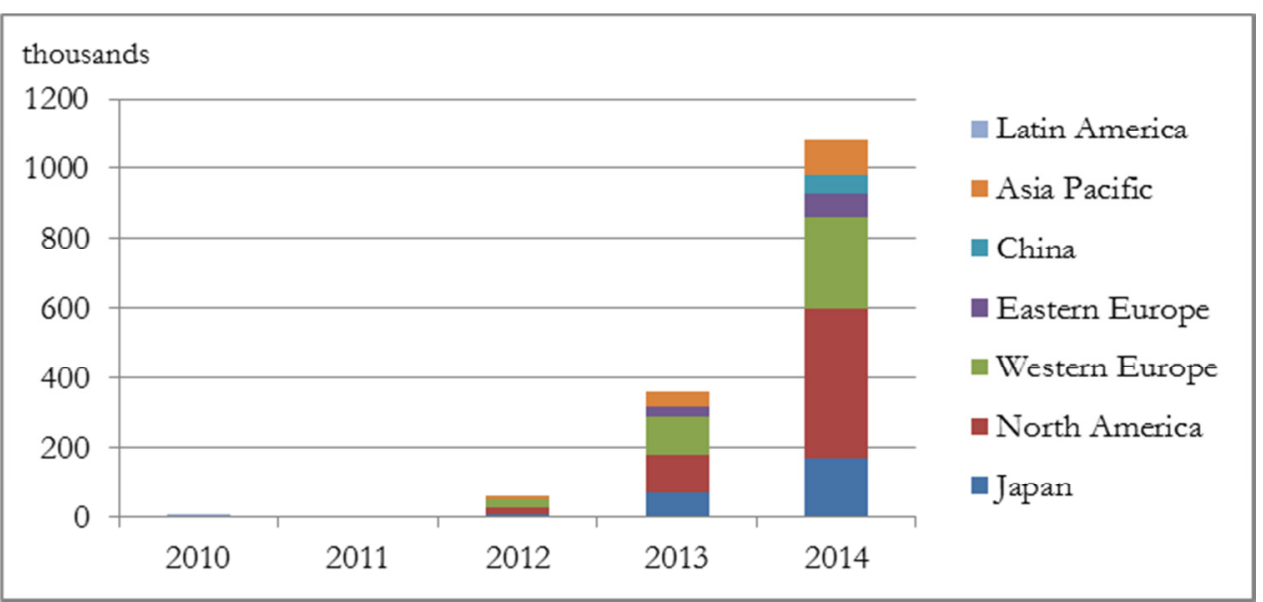

Source: DisplaySearch 2011a

Figure 2-9. OLED TV Shipment Forecast by Region

20 Color gamut is a defined complete set of colors commonly represented as areas in the CIE 1931 Chromaticity Diagram or defined in National Television System Committee (1987). 


\subsubsection{Emerging Market Forecast: India and Brazil}

\section{A] India}

According to DisplaySearch (2009), total TV shipments for India will increase by about 32\% from 2008 to 2013, reaching 16.7 million units, which represent about 35\% of total predicted TV shipments for the AP region outside of China and Japan. In addition, LCD TVs are expected to overtake CRT TVs in India during the next 2 years. The change will take place particularly for small screen sizes -- between 20 -inch $(50.8-\mathrm{cm})$ and 26-inch $(66-\mathrm{cm})$-- as CRT TVs are replaced. Because the India TV market accounts for 35\% to $40 \%$ of the AP regional market, excluding China and Japan, this trend affects the regional AP market transition toward LCD TVs. Figure 2-10 shows the India TV shipment forecast.

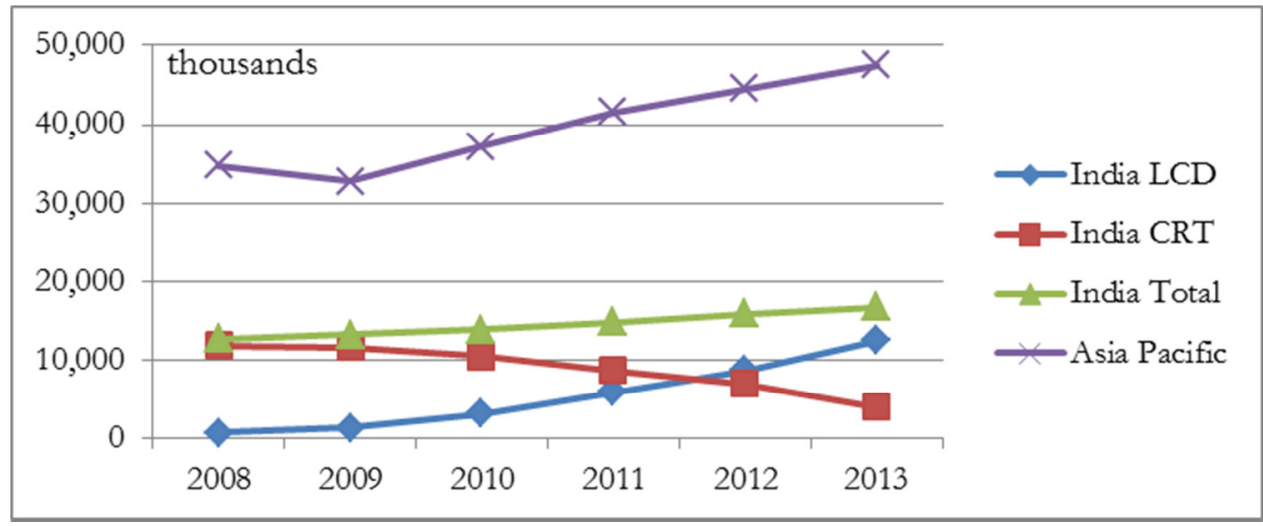

Source: DisplaySearch 2009

\section{Figure 2-10. India TV Shipment Forecast by Technology}

\section{B] Brazil}

Brazil, another emerging market, is expected to face a market transition from CRT TVs to LCD TVs during the next few years that is similar to that predicted in India. Since 2007, LCD TV sales in Brazil have been growing significantly. Annual LCD TV sales are expected to reach about 10 million units in 2013, accounting for about $45 \%$ of the LA regional market. Figure 2-11 shows the Brazil TV sales forecast.

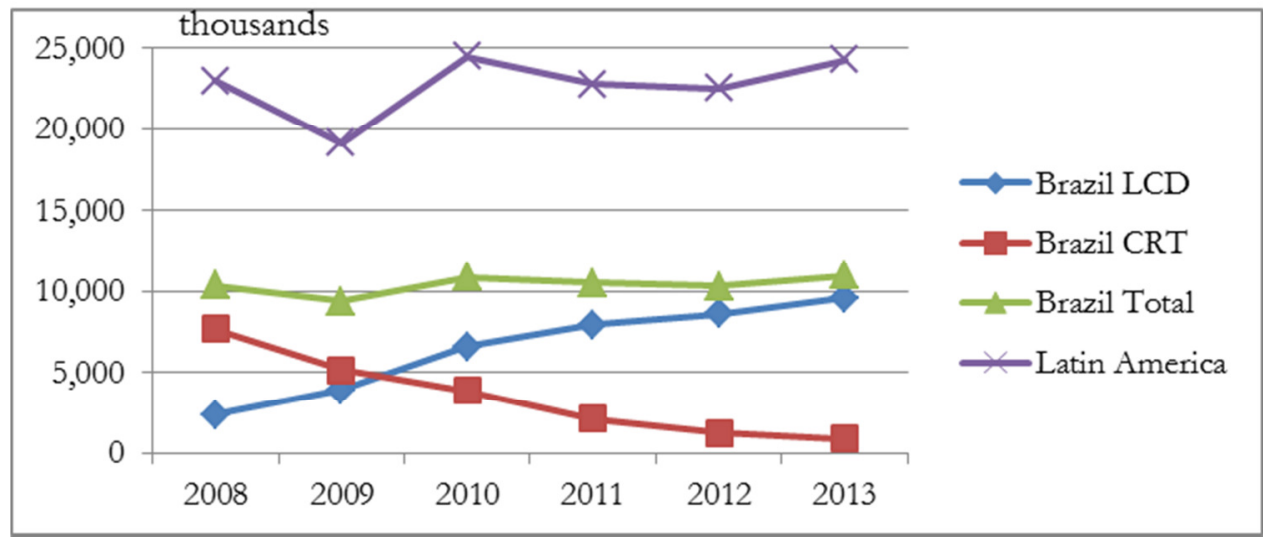

Source: DisplaySearch 2010a

\section{Figure 2-11. Brazil TV Sales Forecast by Technology}

As discussed in Section 2.1, the CRT TV market share is expected to decrease rapidly as major CRT TV manufacturers stop mass production over the next 2 years. To replace this CRT demand, major TV brands have plans to provide affordable LCD TVs, focusing on LED-LCD TVs. 


\subsubsection{Market Forecast by Country}

Display Search's Global TV Shipment and Forecast Report is based on regional TV shipment data and does not provide country-specific TV shipment data except for Japan and China. The authors of this report were not able to refer directly to country-specific TV market data, so some simplifying assumptions were used to estimate TV shipments (or sales) for SEAD-14. Data for Brazil, China, Japan, and India were adopted directly from DisplaySearch $(2009,2011$ a). In accordance with recommendations from DisplaySearch, an estimated $89 \%$ of LCD TVs shipped to North America go to the U.S., and Canada makes up the remaining 11\%. For other countries, country-specific factors are assumed from discussion with manufacturers and experts. The country-specific factors below are applied uniformly to all TV product categories: CRT, LCD PDP, and OLED. Each country's TV shipment (or sales) growth rate from 2010 to 2014 is the same as that of corresponding region. Table 2-3 shows estimated TV shipments (or sales) by country for 2010, and Figure 212 shows shipment (or sales) forecasts for SEAD-14 and China.

\begin{tabular}{l|r|r|r|l}
\hline Country & $\begin{array}{l}\text { Estimated } \\
\text { number of units } \\
\text { in millions }(\mathrm{M})\end{array}$ & $\begin{array}{r}\text { Shipments } \\
\text { or Sales }\end{array}$ & $\begin{array}{l}\text { Assumed } \\
\text { Factor }\end{array}$ & $\begin{array}{l}\text { TV Shipments } \\
\text { from DisplaySearch (2011a) }\end{array}$ \\
\hline Australia & $7.3 \mathrm{M}$ & Sales & 0.2 & Asia Pacific 36.6M \\
\hline Brazil & $10.9 \mathrm{M}$ & Sales & 1 & Brazil \\
\hline Canada & $4.7 \mathrm{M}$ & Shipments & 0.11 & North America 42.7M \\
\hline China & $45.2 \mathrm{M}$ & Shipments & 1 & China 45.2M \\
\hline France & $8.5 \mathrm{M}$ & Sales & 0.2 & Western Europe 42.3M \\
\hline Germany & $9.7 \mathrm{M}$ & Sales & 0.23 & Western Europe 42.3M \\
\hline India & $13.9 \mathrm{M}$ & Shipments & 1 & India 13.9M \\
\hline Japan & $24.1 \mathrm{M}$ & Shipments & 1 & Japan 24.1M \\
\hline Korea & $5.8 \mathrm{M}$ & Sales & 0.16 & Asia Pacific 36.6M \\
\hline Mexico & $5.9 \mathrm{M}$ & Sales & 0.24 & Latin America 24.5M \\
\hline Russia & $7.2 \mathrm{M}$ & Sales & 0.45 & Eastern Europe 15.9M \\
\hline South Africa & $1.4 \mathrm{M}$ & Sales & 0.09 & Middle East \& Africa 15.4M \\
\hline Sweden & $1.3 \mathrm{M}$ & Sales & 0.03 & Western Europe 42.3M \\
\hline UK & $10.6 \mathrm{M}$ & Sales & 0.25 & Western Europe 42.3M \\
\hline USA & $38.0 \mathrm{M}$ & Shipments & 0.89 & North America 42.7M \\
\hline
\end{tabular}

Source: DisplaySearch (2009, 2011a) and author's assumptions based on interviews with marketing experts from the industry

Table 2-3. Estimated 2010 TV Shipments (or Sales) for SEAD-14 and China ${ }^{21}$

\footnotetext{
21 Because of timing factors and different methods of data collection, it is difficult to identify a precise relationship between TV "sell-in" (shipments) and TV "sell-through" (sales) in a region even though these data are closely related to each other. In general, TV shipment data do not necessarily correspond to TVs sold in the given year. TV sales data are usually collected at the point of sale by retailers, but the data may not capture all retail stores or online channels in the region. In addition, several timing factors can skew the relationship between sell-in and sell-through data. For example, a TV set shipped at the end of the year may not arrive at a distributor or a retail store in the same year; as a result, existing stock from previous years may be included in the sale records of any given year. For these reasons, TV shipment and sales data are not directly comparable. Although we should be careful not to mix two types of data sets within one region, in some cases the data set used varies among regions. In Table 2-3, the data for Canada, China, India, Japan, and the U.S. are for TV shipments; the data for other countries are for TV $\underline{\text { sales. }}$
} 


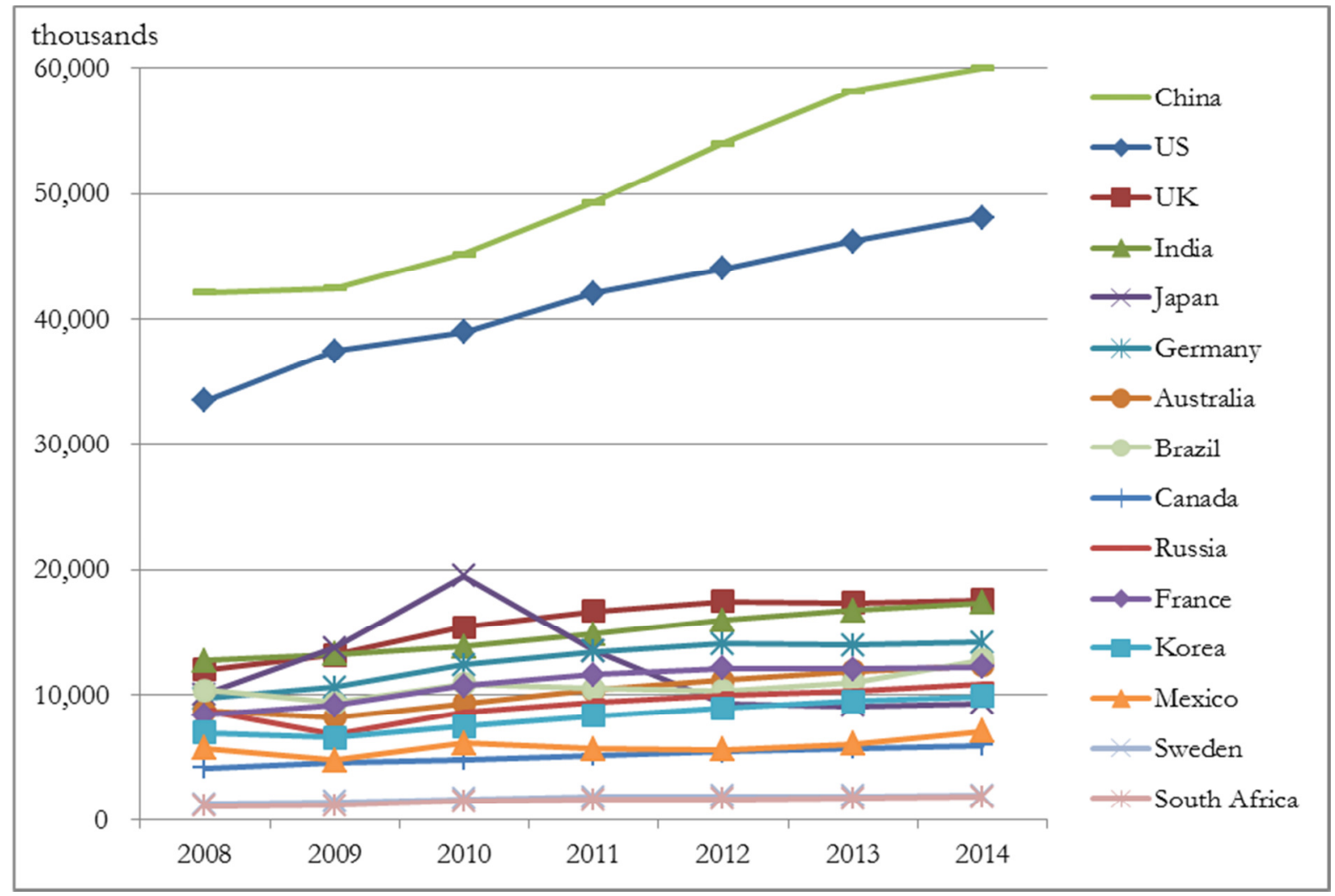

Source: DisplaySearch 2009, 2011a and authors' assumptions

Figure 2-12. TV Shipment (or Sales) Forecast for SEAD-14 and China

Estimates of global and country-specific energy saving potentials will be based on the above TV market forecast data. Details are discussed in Chapter 5, Estimates of Energy Savings Potentials.

\subsection{TV Market Forecast by Screen Size}

Average TV screen size across the globe is expected to increase by $6.5 \%$ from 32.4 inches $(82.3 \mathrm{~cm})$ in 2010 to 34.7 inches $(88.1 \mathrm{~cm})$ in 2014. In some regions such as Europe and China, however, only a small increase in TV sizes from $1.5 \%$ to $2.4 \%$ is expected during this period. Regions such as AP (excluding China), ME\&A, and LA will see a significant increase in screen size from $9.3 \%$ to $21.3 \%$. However, according to DisplaySearch (2011f), screen size preferences in developed regions are expected to stabilize over time because home space is one of the limiting factors for screen size growth. In developing regions, growth in larger screen sizes correlates with economic growth and decrease in the market price of large-sized TVs. At present, 32-inch $(81.3-\mathrm{cm})$ TVs dominate in developed regions, and 46-inch $(116.8-\mathrm{cm})$ and larger sizes have a significant share in NA and China. It is expected that the 40- to 42-inch (101.6- to 106.7-cm) size band will become more popular during the next several years. Developing regions, including AP, LA, and ME\&A, have historically been dominated by 20 - to 21 -inch $(50.8-$ to $53.3-\mathrm{cm}$ ) CRT TVs, but these regions are expected to experience shifts in average screen size from 20-21-inch $(50.8-53.3-\mathrm{cm})$ band to 32 -inch $(81.3-\mathrm{cm})$ with the rapid penetration of LCD TVs. Figure 2-13 shows a recent forecast for screen size trend by region. 


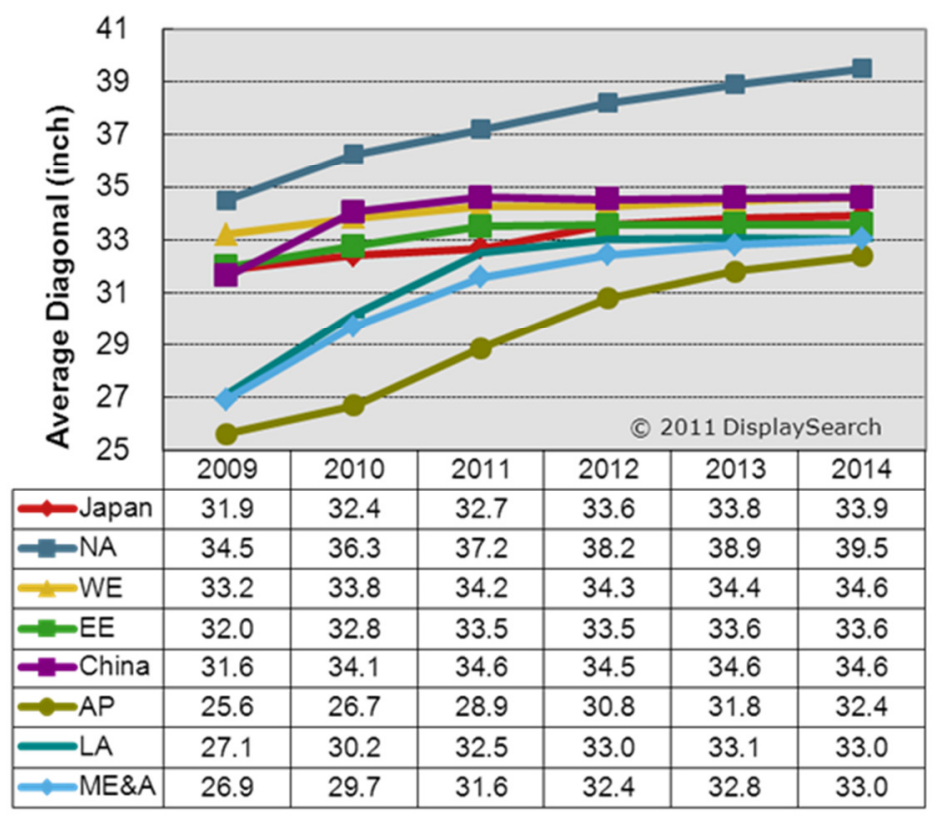

Source: DisplaySearch 2011f

\section{Figure 2-13. Screen Size Trend by Region}

\subsubsection{Screen Size Increase and TV Power Consumption}

The average energy consumption per TV is expected to increase with progressive increases in the average screen size of TVs sold. According to DisplaySearch (2011f), in 2010 the total display area by total TV shipments (248 million units) was 79 million $\mathrm{m}^{2}\left(0.32 \mathrm{~m}^{2}\right.$ per unit). The total display area in 2014 is expected to be $104 \mathrm{~m}^{2}$ for 292 million units $\left(0.35 \mathrm{~m}^{2}\right.$ per unit). Figure 2-14 shows average screen area per unit and global TV shipments by technology for 2010 and 2014.
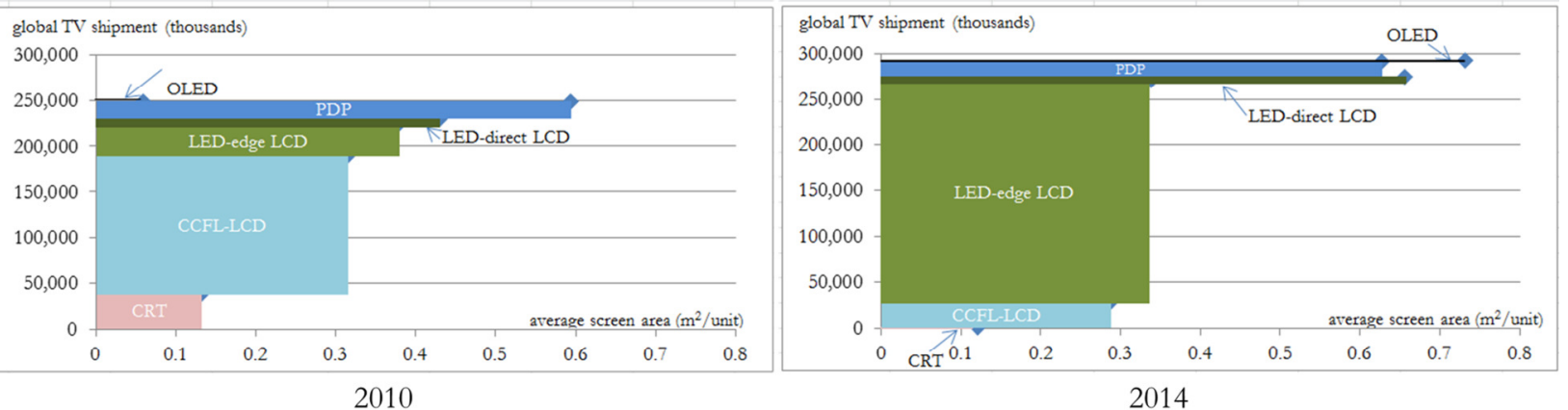

Source: Author's calculation based on DisplaySearch 2011f

\section{Figure 2-14. Global TV Shipments and Average Screen Area by Technology for 2010 and 2014}

Based on the average on-mode power of 2010 TV models, we can analyze the impact of increases in screen size and TV sales on TV power consumption. Although the average screen size and area for all technologies are expected to increase, the average screen area for LED-LCD TVs is expected to decrease as the market share increases in smaller screen sizes. Because LED-LCD TVs are expected to dominate the market and are more efficient than CCFL-LCD TVs, it seems that the impact of increase in screen size and TV sales on the total on-mode power is not very significant, from 17.9 GW in 2010 to $18.7 \mathrm{GW}$ in 2014. As a result, the large-scale transition from CCFL-LCD TVs to LED-LCD TVs are expected to reduce the impact of 
countervailing trends in screen size and TV sales on total TV power consumption. For OLED TVs, the average screen size forecast at the early stage has not been certain. A recent DisplaySearch report (DisplaySearch 2011f) expected that 40- to 55-inch OLED TVs will be realized from the year 2012 forward, while the previous reports from DisplaySearch forecasted that 32- to 40-inch OLED TVs would be commercialized in 2012. At the early stage, it is likely that manufacturers will provide OLED TVs over 40 inches for flagship models. Table 2-4 shows the average screen area per unit and average on-mode power by technology, assuming that there will be no efficiency improvement up to 2014. Figure 2-15 shows the total on-mode power consumption multiplied by total TV shipments.

\begin{tabular}{l|r|l|r|r|r|r}
\hline & \multicolumn{3}{|c|}{2010} & \multicolumn{3}{|c}{2014} \\
\hline & EEI & $\begin{array}{l}\text { Average } \\
\text { screen area } \\
\text { per unit } \\
\left(\mathrm{m}^{2} / \text { unit }\right)\end{array}$ & $\begin{array}{l}\text { Average on- } \\
\text { mode Power }\end{array}$ & EEI & $\begin{array}{l}\text { Average } \\
\text { screen area } \\
\text { per unit } \\
\left(\mathrm{m}^{2} / \text { unit }\right)\end{array}$ & $\begin{array}{l}\text { Average on- } \\
\text { mode Power }\end{array}$ \\
\hline OLED & 0.247 & 0.06 & $11 \mathrm{~W}$ & 0.247 & 0.73 & $83 \mathrm{~W}$ \\
\hline PDP & 0.436 & 0.59 & $120 \mathrm{~W}$ & 0.436 & 0.63 & $127 \mathrm{~W}$ \\
\hline LED-LCD & 0.356 & 0.39 & $67 \mathrm{~W}$ & 0.356 & 0.34 & $59 \mathrm{~W}$ \\
\hline CCFL-LCD & 0.467 & 0.31 & $72 \mathrm{~W}$ & 0.467 & 0.29 & $68 \mathrm{~W}$ \\
\hline CRT & 0.725 & 0.13 & $55 \mathrm{~W}$ & 0.725 & 0.12 & $52 \mathrm{~W}$ \\
\hline
\end{tabular}

Source: Author's calculation based on ENERGY STAR 2011a and DisplaySearch 2011f

Table 2-4. Average Screen Area per Unit and Average On-Mode Power for Technologies without Efficiency Improvement

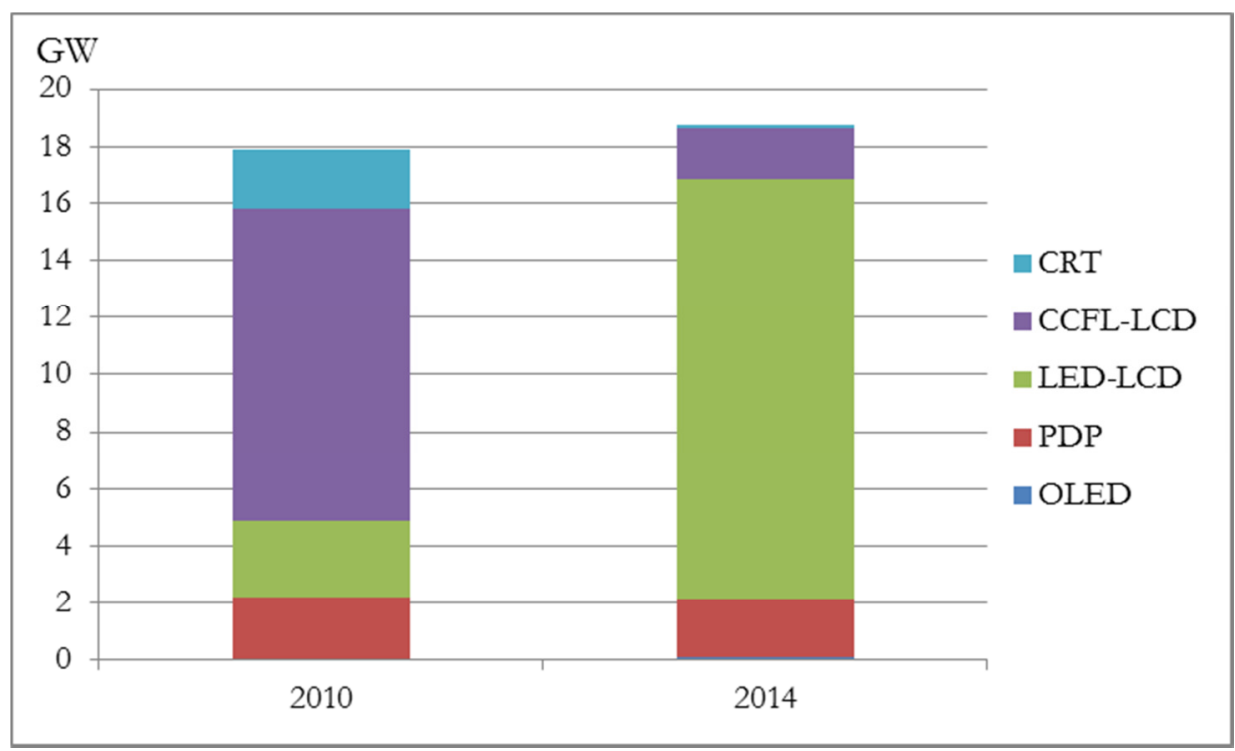

Source: Author's calculation based on ENERGY STAR 2011a and DisplaySearch 2011f

Figure 2-15. Total On-Mode Power by TV Market Forecast without Efficiency Improvement

If we apply the base-case efficiency scenario used in this report, the average on-mode power for each technology decreases, and the total on-mode power by new TV shipments also decreases from $17.9 \mathrm{GW}$ in 2010 to $12.7 \mathrm{GW}$ in 2014. As a result, combination of large-scale transition between LCD backlight technologies along with efficiency improvements will negate the impact of countervailing trends in screen size and TV sales on total TV power consumption. Table 2-5 shows average display area per unit and average on-mode power forecast with efficiency improvement. 


\begin{tabular}{l|r|l|r|r|r|r}
\hline & \multicolumn{3}{|c|}{2010} & \multicolumn{3}{c}{2014} \\
\hline & EEI & $\begin{array}{l}\text { Average } \\
\text { screen area } \\
\text { per unit } \\
\left(\mathrm{m}^{2} / \text { unit }\right)\end{array}$ & $\begin{array}{l}\text { Average on- } \\
\text { mode Power }\end{array}$ & EEI & $\begin{array}{l}\text { Average } \\
\text { screen area } \\
\text { per unit } \\
\left(\mathrm{m}^{2} / \text { unit }\right)\end{array}$ & $\begin{array}{l}\text { Average on- } \\
\text { mode Power }\end{array}$ \\
\hline OLED & 0.247 & 0.06 & $11 \mathrm{~W}$ & 0.183 & 0.73 & $61 \mathrm{~W}$ \\
\hline PDP & 0.436 & 0.59 & $120 \mathrm{~W}$ & 0.314 & 0.63 & $92 \mathrm{~W}$ \\
\hline LED-LCD & 0.356 & 0.39 & $67 \mathrm{~W}$ & 0.239 & 0.34 & $40 \mathrm{~W}$ \\
\hline CCFL-LCD & 0.467 & 0.31 & $72 \mathrm{~W}$ & 0.322 & 0.29 & $47 \mathrm{~W}$ \\
\hline CRT & 0.725 & 0.13 & $55 \mathrm{~W}$ & 0.653 & 0.12 & $47 \mathrm{~W}$ \\
\hline
\end{tabular}

Source: Author's calculation based on ENERGY STAR 2011a and DisplaySearch 2011f

Table 2-5. Average Display Area per Unit and Average On-Mode Power for Technologies with Efficiency Improvement

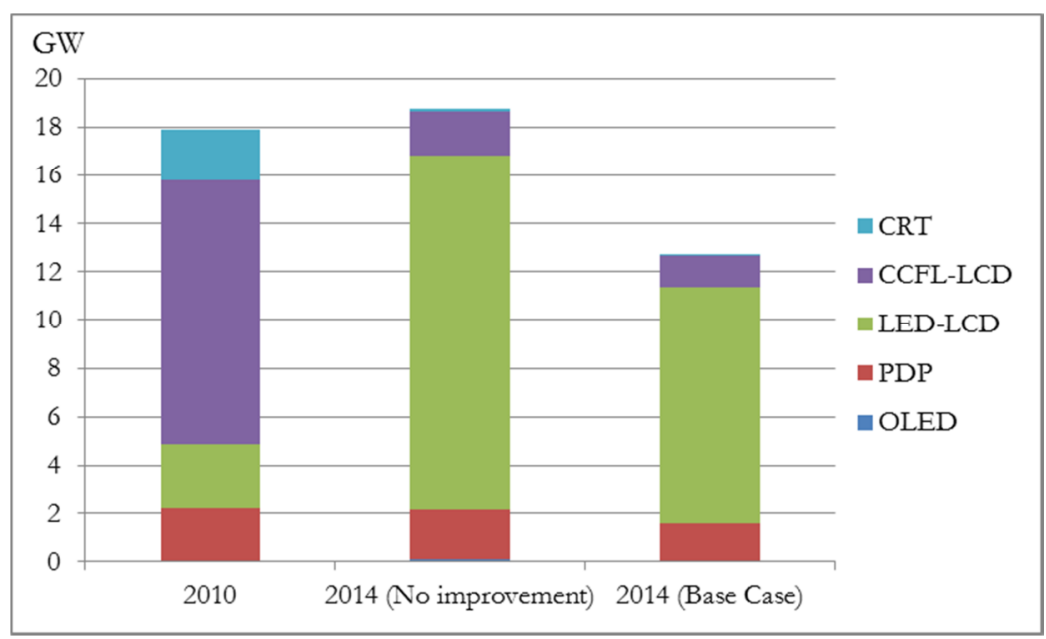

Source: Author's calculation

Figure 2-16. Total On-Mode Power by TV Market Forecast with Efficiency Improvement

\section{Impact of Standards on Countervailing Screen Size and TV Sales}

Progressive increase in the size of TVs sold is expected to increase average TV power consumption. In the absence of efficiency improvements, average TV power consumption would increase by about $10 \%$ as a result of the expected 7\% increase in screen size between 2010 and 2014. The maximum TV power consumption allowed under ENERGY STAR Version 4 criteria is shown in Table 2-6.

\begin{tabular}{l|r|r|r}
\hline & 2010 & 2014 & $\begin{array}{l}\text { \% change from } \\
2010 \text { to } 2014\end{array}$ \\
\hline Average Screen Size (worldwide) & 32.4 inches & 34.7 inches & $7.0 \%$ \\
\hline Average Screen Area (worldwide) & 448.2 inch $^{2}$ & $514.1 \mathrm{inch}^{2}$ & $14.7 \%$ \\
\hline U.S. ENERGY STAR Version 4 & $\mathrm{P}_{\max }{ }^{*}=79 \mathrm{~W}$ & $\mathrm{P}_{\max }{ }^{*}=87 \mathrm{~W}$ & $10.0 \%$ \\
\hline
\end{tabular}

$P_{\max } *=0.120 \times A+25$, (A: sq. in., $A \geq 275$ )

Table 2-6. Increase in TV Power Consumption Corresponding to Predicted Increase in Screen Size

However, new efficiency standards such as ENERGY STAR Version 5 are expected to negate the effect of TV screen size increase on energy consumption. Assuming that all TVs that are/will be shipped to NA will meet the highest ENERGY STAR criteria for their screen size, annual energy consumption for total TV shipments can be estimated as shown in Figure 2-17. If we assume that all TVs sold in North America from 
2012 to 2014 qualify for ENERGY STAR Version 5, the annual TV energy consumption from 2010 to 2014 is expected to be lower than the case in which all TVs qualify only for ENERGY STAR Version 4, despite expected increases in screen size and TV sales.

\begin{tabular}{l|r|r|r}
\hline & \multicolumn{1}{|c|}{2010} & 2012 & \multicolumn{1}{c}{2014} \\
\hline Average Screen Size (North America) & 36.0 inches & 37.8 inches & 39.2 inches \\
\hline Average Screen Area (North America) & 553.4 inch $^{2}$ & 610.1 inch $^{2}$ & 656.1 inch $^{2}$ \\
\hline TV Shipments (million units) & 42.7 & 47.7 & 52.4 \\
\hline U.S. ENERGY STAR VERSION 4 & $\mathrm{P}_{\max } *=91.4 \mathrm{~W}$ & - & - \\
\hline U.S. ENERGY STAR VERSION 5 & - & $\mathrm{P}_{\max } * *=69.2 \mathrm{~W}$ & $\mathrm{P}_{\max } * *=73.1 \mathrm{~W}$ \\
\hline
\end{tabular}

$\mathrm{P}_{\max } *=0.120 \times \mathrm{A}+25$, (A: sq. in., $\left.\mathrm{A} \geq 275\right), \mathrm{P}_{\max } * *=0.084 \times \mathrm{A}+18$ (A: sq. in., $275 \leq \mathrm{A} \leq 1,068$ )

Source for average screen size and TV shipments: DisplaySearch 2011a

\section{Table 2-7. Increase in Screen Size and TV Shipments for North America}

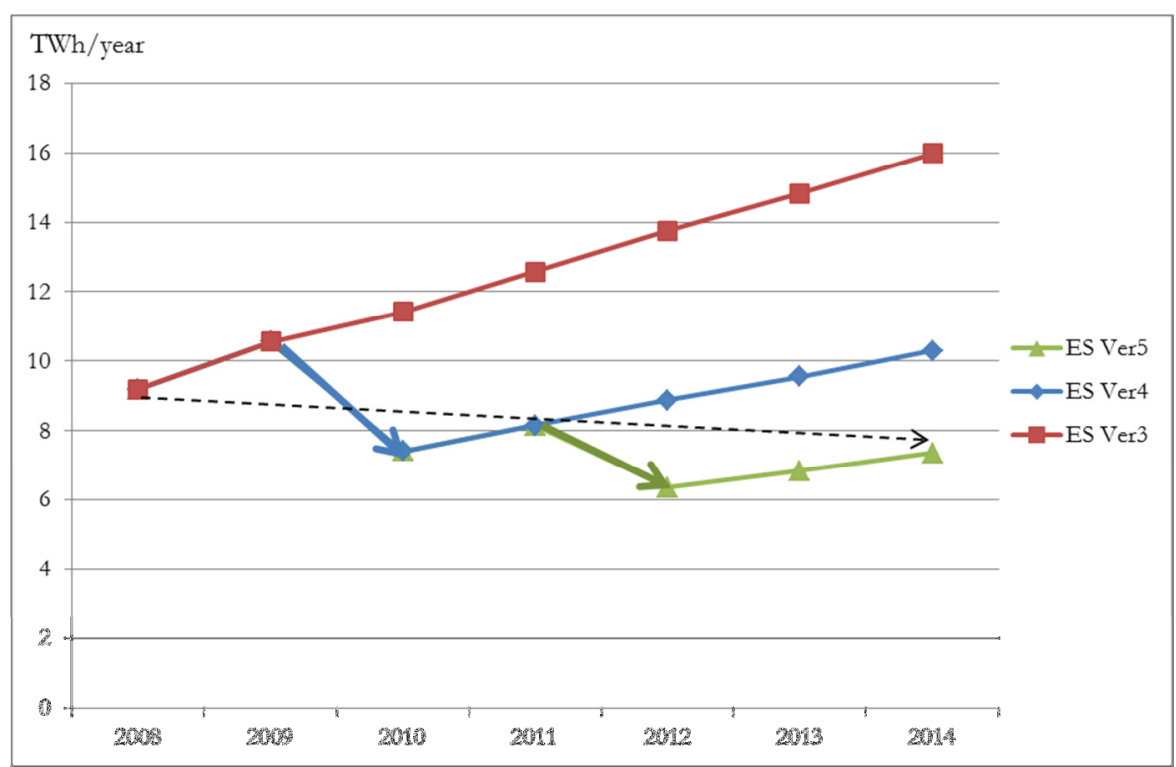

Figure 2-17. Effect of Forecast Screen Size and North America TV Shipments on Energy Consumption $^{22}$

22 It is assumed that average daily viewing time is 5 hours, and average standby power is $1 \mathrm{~W}$. 


\section{TV Technology Trends and Energy Consumption}

Two recent significant TV technology trends are: 1) adoption of 3D technology, and 2) development of internet-enabled TVs with advanced functions, also known as Smart TVs.

3D TV technologies are not yet mature; they require the viewer to wear glasses, and 3D content is not widely available. However, since large-size (40"+) FPD TVs were commercialized in the early 2000s, both LCD and PDP TVs have overcome image quality issues, allowing 3D technology to become a new technical feature that manufacturers are using to market their products. 3D TVs are still expensive and mainly used as flagship products. However, experts and manufacturers expect the technology to continue to improve and to be available in entry- or mid-level models in the near future. Current 3D LCD TVs require high refresh rates over 200/240 Hertz (Hz) to successfully generate coherent 3D images. In general, $60-80 \%$ of light is lost in $3 \mathrm{D}$ mode of a 3D-capable $\mathrm{TV}$, when compared to the luminance in $2 \mathrm{D}$ mode. This loss may result in brightness perceived by the viewer in 3D mode being lower than the brightness perceived in 2D mode, although the actual luminance of the screen may stay the same or above for a constant effective brightness in 3D mode. ${ }^{23}$ As 3D perception renders the image subjectively brighter than it technically is, anecdotal evidence from manufacturers suggests that the minimum luminance level required for an image formed by a $3 \mathrm{D}$ TV to be acceptable to viewers in a dark room is expected to be at least 90 candelas per square meter $\left(\mathrm{cd} / \mathrm{m}^{2}\right)$ while the maximum luminance currently achieved by typical 2D-only LCD TVs is between 400 and $500 \mathrm{~cd} / \mathrm{m}^{2}$ (Chung 2010). Some of existing 3D TVs automatically detect and boost brightness when 3D content is displayed. This does not imply that 3D capable TVs use more energy than $2 \mathrm{D}$ TVs when viewing 2D images, it is in 3D mode that additional energy is used. However, 3D content available to consumers is still limited, which makes it hard to project viewing hours for 3D content. Also, manufacturers and experts expect such issues to be overcome in a few years as both 3D technologies and screen efficiency are improved over time.

Connected TVs (or Smart TVs) require additional features for network connectivity and advanced hardware components such as a central processing unit (CPU), graphical processing unit (GPU) and memory; these components may increase the unit's power by a few watts. Increased penetration of connected TVs may encourage consumers to buy larger screens and extend viewing hours in order to enjoy screens with multiple functions, which will also increase TV energy consumption. In addition, local dimming methods will not be very useful in lowering power consumption for white internet screens. In addition to on-mode power, connected TVs have the potential to increase standby mode power, allowing them to keep ready to be turned on within a few seconds. As a result, if both 3D TV and connected TV functions are incorporated into entrylevel TV models, the resulting increased energy will to some degree offset consumption efficiency improvements in TVs, as summarized in Table 3-1.

\footnotetext{
${ }^{23}$ Luminance refers to a photometric measure of the luminous intensity of light per unit area travelling in a given area, a measured value from a photo-detector described in a form of candela per square meters $\left(\mathrm{cd} / \mathrm{m}^{2}\right)$. Brightness is a subjective attribute of visual perception elicited by the luminance of an object. It does not necessarily correlate with luminance in a linear scale. See more details in Appendix E.
} 


\begin{tabular}{|c|c|c|}
\hline & On-Mode Power (watts) & Total Energy Consumption (watt-hours) \\
\hline 3D TV & $\begin{array}{l}(+) \text { advanced hardware for } 3 \mathrm{D} \text { image } \\
\text { processing } \\
(+) \text { high refresh rate over } 200 / 240 \mathrm{~Hz} \\
(+) \text { compensation for decreased brightness }\end{array}$ & $\begin{array}{l}(+) \text { viewing hours for 3D content } \\
\text { (uncertain) }\end{array}$ \\
\hline $\begin{array}{l}\text { Smart TV } \\
\text { (Connected TV) }\end{array}$ & $\begin{array}{l}(+) \text { advanced hardware in CPU, GPU, and } \\
\text { memory } \\
(+) \text { large and wide screen }(16: 9 \rightarrow 20: 9) \\
(+) \text { white internet screens } \\
(-) \text { black or dark internet screens(with } \\
\text { dimming techniques) }\end{array}$ & $\begin{array}{l}\text { (+) possible increase in usage due to } \\
\text { multiple functions } \\
(+) \text { increase in web server usage } \\
(+) \text { increase in standby power use with } \\
\text { fast start options } \\
(+) \text { increase in network standby power } \\
\text { use }\end{array}$ \\
\hline
\end{tabular}

Table 3-1. Effect of 3D TV and Smart TV on Unit Efficiency and Energy Consumption

\subsection{D TV}

In the following subsections, we discuss the current status of and future forecast for 3D TVs in the overall TV market, the status of current 3D technology, the direction of future development in 3D technology, and the likely impact of 3D technology on TV energy consumption.

\subsubsection{D T'V Market}

Since the concept of stereoscopic image formation was introduced and a mirror stereoscope viewer was developed in 1838, the broader 3D industry has been growing slowly. ${ }^{24}$ Recent successes of 3D movies have whetted consumers' appetites for the 3D experience and catalyzed the expansion of the 3D market to TV (Chung 2010). Although only limited 3D content is available to TV consumers, FPD TV penetration in new TV sales is nearly $100 \%$ in developed regions (DisplaySearch 2010c), and TV retail prices are continuing to fall. In view of these trends, 3D TV may be used by manufacturers to boost TV sales in these regions. Growth in the 3D TV market will need to be accompanied by available content and internet connectivity improvements. Although 3D broadcasting was scheduled to begin after 2010 in some countries, development of $3 \mathrm{D}$ content will require further investment.

According to DisplaySearch (2011d), 3D TV demand is expected to be 3.2 million units in 2010 and 91.5 million units in 2014, which is a 32\% share of the global TV shipments forecast by DisplaySearch for 2014. Because the 3D TV market is at an early stage and the corresponding technologies are not yet mature, these forecasts are uncertain. Table 3-2 shows the 3D TV forecast for 2010 to 2014.

24 Stereoscopic image formation refers to the phenomenon in which the human brain creates a perception of depth (necessary for 3D) from a composite of the images seen by the left and right eyes. 


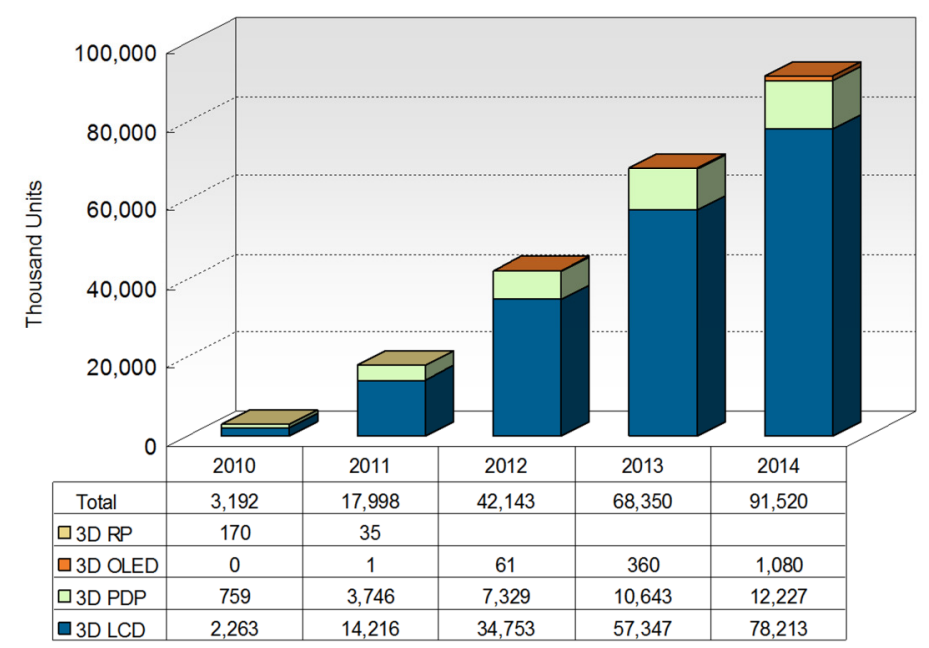

*RP: rear projection

Source: DisplaySearch 2011d

\section{Table 3-2. Global 3D TV Forecast by Technology}

In the short term, PDPs appear to have an advantage in the 3D market because they have fewer picture quality problems ${ }^{25}$ and have a 3D panel price similar to the price of 2D-only panels, compared with LCDs. Demand for PDP 3D TVs was 0.76 million units in 2010 and expected to grow continuously to 12.2 million units in 2014, mainly driven by growth in larger screen sizes. However, the PDP industry's overall market share is declining, so it is expected that the 3D PDP expansion will be limited although the proportion of 3D TVs within the PDP market will be increasing. Table 3-3 shows the 3D TV market share by technology and screen size.

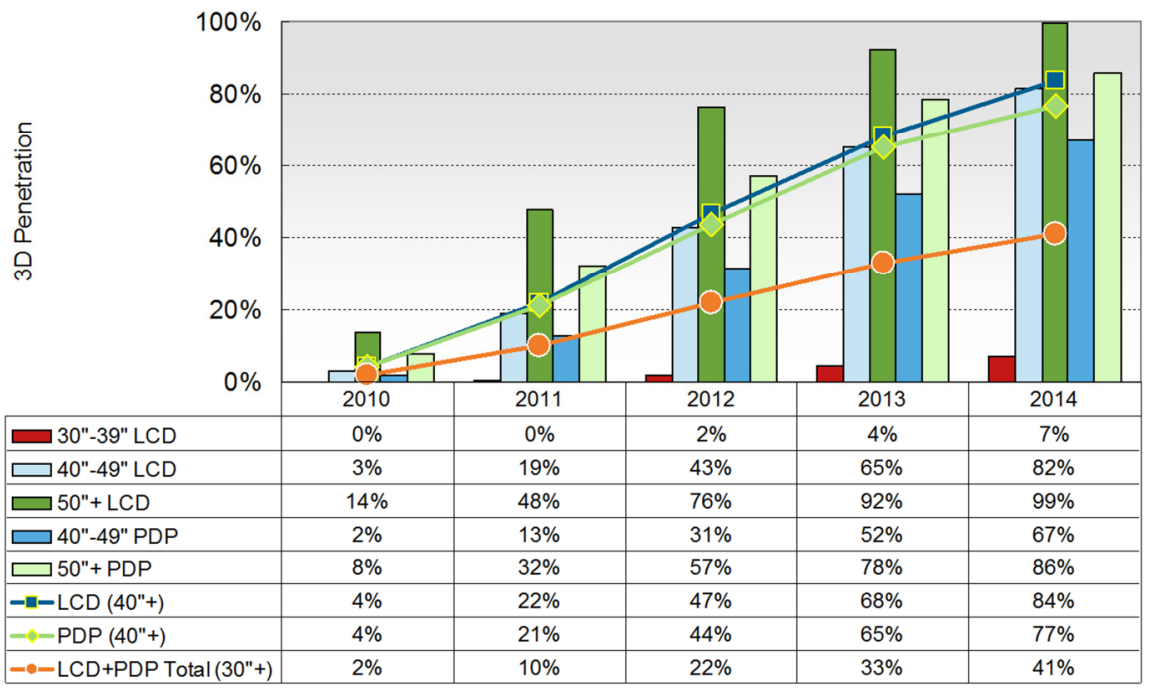

Source: DisplaySearch 2011d

Table 3-3. Global 3D TV Market Penetration by Technology and Screen Size

25 In addition to decrease in brightness caused by filters or glasses, two other issues with 3D TVs are: 1) Flicker, which occurs when screens are driven at low refresh rate, allowing the brightness to drop for intervals noticeable by the human eye. Because 3D displays split and reconstruct images, current LCD TVs must be driven at higher refresh rate, e.g., 200/240Hz. 2) Crosstalk, which refers to the overlapping of the image for the left eye with that for the right eye and vice versa. This is also caused by the slow response time of a liquid crystal display. 
3D LCD TVs require a high refresh rate, e.g., 200/240Hz, with correspondingly higher material cost than LCD panels which have lower refresh rates. Manufacturers expect LCD technology to overcome the current technical limitations in the near future. DisplaySearch (2011d) projects that the global demand for 3D LCD TVs will reach 34.8 million units in 2012 and continue growing to 78.2 million units in 2014. OLED TVs could have a significant impact on the 3D TV market when compared to LCD and PDP TVs because OLED is a self-emissive display (like PDP) and has much faster response time than LC materials. However, because the OLED technology is still immature and expensive, it is difficult to predict the future share that OLED 3D TVs will command in the overall 3D TV market.

\subsubsection{D TV Technology}

3D technology has evolved from its inception as anaglyph technology to the currently available stereoscopic technology, both of which require viewers to wear glasses. As technical limitations are overcome, this technology is expected to continue to evolve, as shown in Table 3-4, toward auto-stereoscopic technology (without glasses) and finally holography, which is closest to what might be termed real 3D. ${ }^{26}$ Major TV manufacturers have already demonstrated auto-stereoscopic 3D TVs, and Toshiba plans to announce availability and pricing for its glass-free 3D TVs in the U.S. market later in 2011 (Toshiba 2011). However, most manufacturers expect that glass-free 3D TVs with good performance ${ }^{27}$ will not be available in the market for a few more years. Table 3-4 lays out the past, present, and future of 3D TV technology.

\begin{tabular}{l|l|l|l}
\hline Past & Present & Future \\
\hline Anaglyph & Stereoscopic & Auto-stereoscopic & Holography \\
\hline Red/Blue glasses & Glasses type & Non-glasses type & Realistic 3D \\
& Polarized / Shutter & $\begin{array}{l}\text { Lenticular lens } \\
\text { (3D only) }\end{array}$ & Amplitude and object \\
& 2D $/ 3 \mathrm{D}$ switchable reproduced \\
& & Switchable lens & \\
\hline
\end{tabular}

Source: Choi 2010

\section{Table 3-4. 3D TV Technology Roadmap}

Broadly, there are two types of stereoscopic technology, as shown in Figure 3-1, based on whether the left-eye and right-eye images are alternated spatially or temporally, and based on whether the viewer's glasses are "active" or "passive." Passive polarized glass technology (with spatially alternated images) is implemented by attaching a polarization film to the surface of the LCD screen, which results in alternating rows of the pixels displaying two different polarized images. In other words, the screen displays the left-eye images in the odd rows and the right-eye images in the even rows. This technology halves the vertical resolution and requires additional cost for polarized films while keeping brightness level fairly high, with fewer image quality problems than other technologies. Active shutter glass technology (with temporally alternated images) is implemented by storing left and right frames and interleaving them temporally using a frame rate conversion device. Active shutter glasses do not entail any additional costs for the 3D screen itself, and resolution is not diminished with this technology. However, the glasses need extra circuitry and a battery, and $70-80 \%$ of light loss occurs at present with this technology, compared to 2D mode.

\footnotetext{
${ }^{26}$ Holography allows the light scattered from an object to be recorded and later reconstructed as so that the original object reappears. Although it is possible to create a hologram of a static object or moving scenes, this technology needs further development and more time to be qualified for TV applications.

27 According to a manufacturer, it is required for glass-free 3D TVs to have viewers enjoy 3D images at various viewing angles, more than 32 spots, in order to be realized in the market.
} 


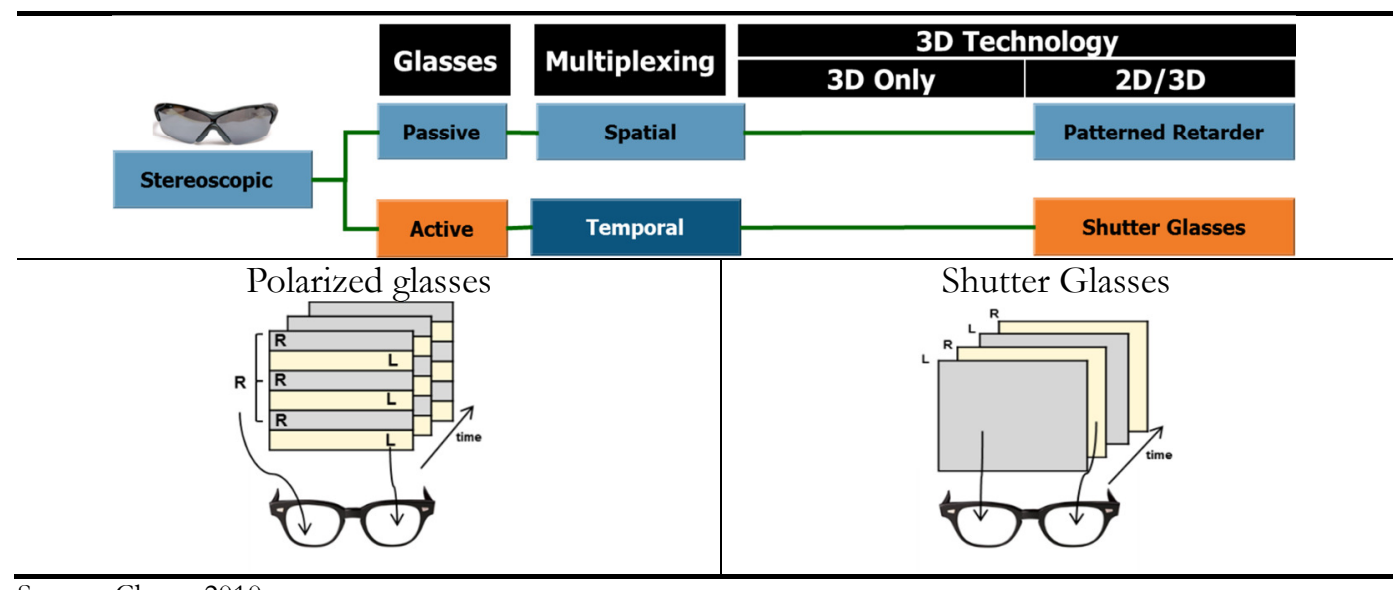

Source: Chung 2010

Figure 3-1. Stereoscopic Technologies for 3D TV

Nevertheless, active shutter glass technology is expected to become more dominant than passive polarized glass technology in 3D TVs during the next 2 to 3 years. While it is difficult to determine which technology is better than the other, major manufacturers have their own technical and economic preferences. Samsung, Sharp, and Sony have been focused on active shutter glass technology, while LG has been leading the development of passive polarized glass technology. Since active shutter glass technology needs to overcome issues with lower brightness, compared to passive polarized glasses, we limit our 3D TV discussion to active shutter glass stereoscopic 3D TVs in this report.

\subsubsection{D TV Energy Consumption and Efficiency Improvement Potentials}

An existing TV in 3D mode is likely to consume more energy than in 2D mode because of the following differences between 3D and 2D technology. First, a 3D-enabled TV includes an additional 3D-image processor on the image circuit board, which consumes 3-5 W. Second, perceived brightness ${ }^{28}$ in 3D mode is less than in 2D mode at present due to light losses from glasses, as outlined in Table 3-5. Although manufacturers have adopted some techniques to enhance brightness in 3D mode, this increase does not allow images that are sufficiently bright for viewing in 3D mode. For this reason, some of current 3D-enabled TVs increase screen luminance in 3D mode by consuming more power to allow a higher "effective brightness" in the viewer's perception. Based on our findings, we estimate that additional power requirement for 3D mode of a few 42" LCD TVs is about 10-20W. According to an expert comment ${ }^{29}$, the power difference between 2D and 3D modes is up to $20 \mathrm{~W}$ for 42-inch LCD and PDP TVs. Table 3-5 shows a conceptual example of LCD 3D TV brightness loss with shutter glasses.

\begin{tabular}{l|l|l|l|l|l}
\hline & $\begin{array}{l}\text { Original } \\
\text { images }\end{array}$ & $\begin{array}{l}\text { Loss from reflection } \\
\text { on screen glass }\end{array}$ & $\begin{array}{l}\text { Separation of } \\
\text { right } / \text { left images }\end{array}$ & $\begin{array}{l}\text { Transmittance of } \\
\text { glasses }\end{array}$ & Final images \\
\hline luminance & $500 \mathrm{~cd} / \mathrm{m}^{2}(100 \%) \rightarrow 450 \mathrm{~cd} / \mathrm{m}^{2}(90 \%) \rightarrow \quad 225 \mathrm{~cd} / \mathrm{m}^{2}(45 \%) \rightarrow \quad \rightarrow \quad 0 \mathrm{~cd} / \mathrm{m}^{2}(18 \%)$ \\
\hline
\end{tabular}

Source: Chung 2010

Table 3-5. Example of Light Loss in 3D mode of 3D LCD TV with Shutter Glasses

\footnotetext{
28 According to manufacturers and Chung (2010), 60-80\% of light loss occurs in 3D mode of a 3D-capable TV, compared to the luminance in 2D mode. 3D TVs with shutter glasses caused an $80 \%$ decrease, while 3D TVs with film patterned retarders caused a $60 \%$ decrease. OLED TV manufacturers claim that brightness of an OLED TV in 3D mode is $60 \%$ lower than in $2 \mathrm{D}$ mode, e.g., $200 \mathrm{~cd} / \mathrm{m}^{2}$ (2D normal mode) and $80 \mathrm{~cd} / \mathrm{m}^{2}$ (3D normal mode). For this reason, viewers may experience relatively low brightness level in 3D mode compared to 2D mode, although 3D perception renders the image subjectively brighter than it technically is.

29 Bob Harrison of Intertek UK
} 
While technological development in 3D technologies and efficiency improvement in both LCD screen and backlights will help manufacturers eventually overcome this issue, LCD TV manufacturers are considering the following alternatives in the short term: high-efficiency optical films to ensure at least minimally acceptable brightness in 3D mode or more backlight lamps. Although both alternatives entail incremental costs, additional backlight lamps are likely to increase power consumption. Some of existing 3D TVs automatically detect and boost brightness when 3D content is displayed, others don't. The first case does not necessarily imply that 3D TVs use more energy when viewing 2D images. It is in 3D mode that additional energy is used. However, 3D content available to consumers is still limited, and it is expected that such issues will be overcome in a few years as both $3 \mathrm{D}$ technologies and screen efficiency are improved over time.

As mentioned earlier, the power difference between 2D and 3D modes for current 42-inch $(106.7-\mathrm{cm}) 3 \mathrm{D}$ TVs is up to $20 \mathrm{~W}$, although it varies with models. More than $90 \%$ of $3 \mathrm{D}$ TVs are expected to have large screen sizes over 40 inches. If we assume that 3D TV purchasers consume 2 hours every day for 3D content and additional power consumption for the $3 \mathrm{D}$ mode is $20 \mathrm{~W}$ for all $3 \mathrm{D}$ TVs, the annual energy consumption by new 3D TV sales in 2014 is estimated to be about 1.2 TWh, and the cumulative energy consumption by new 3D TV sales from 2010 to 2014 is about 3 TWh. 40- to 49-inch (101.6- to 124.5-cm) LCD TVs will account for about $70 \%$ of the cumulative energy consumption. Figure 3-2 shows that estimates of potential energy consumption additionally consumed at maximum level $(20 \mathrm{~W})$ in $3 \mathrm{D}$ mode of 3D TVs.

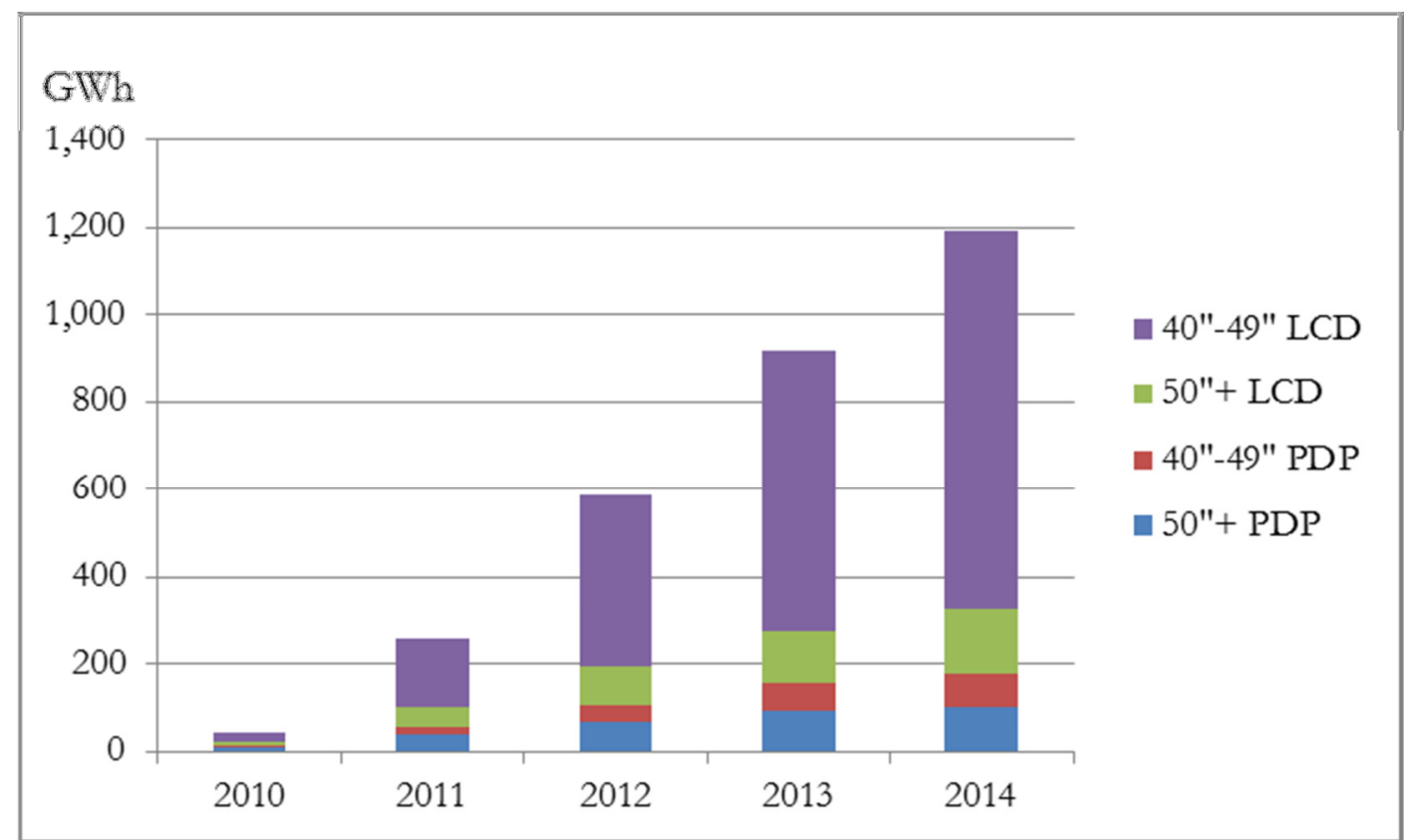

Figure 3-2 Estimates of Annual Energy Consumption in 3D mode of 3D TVs by forecasted Global TV Sales

3D LCD TVs require a high refresh rate (e.g., 200/240 Hz), which consumes more power than the low refresh rate of LCD TVs. High refresh rates decrease luminance by lowering the LCD panel's transmittance. Some manufacturers are developing $120 \mathrm{~Hz}$ 3D TVs to overcome the issues of low brightness and high cost. The overall magnitude of the impact on energy consumption of the shift from 2D to 3D mode is dependent on manufacturers' strategies to increase brightness and users' subjective tastes, e.g., changes in consumers' viewing time for $3 \mathrm{D}$ content. It is, therefore, difficult to estimate precisely. 


\subsection{Smart TV (Connected TV)}

The following subsections discuss smart TV (or connected TV) markets and energy consumption. DisplaySearch defines a connected TV as a TV with an internet or network connection. (DisplaySearch 2011e) Smart TV, sometimes described as connected TV or hybrid TV, is the phrase used to describe the current trend of television sets with integrated internet capabilities. (Wikipedia 2011a) In Korea, the Ministry of Knowledge Economy has differentiated smart TV system from conventional TV system by allowing smart TV manufacturers to obtain broadcasting contents directly from content producers while in the conventional system broadcasters get programs from content producers and deliver them to viewers (MKE 2010). Although smart TVs are regarded as connected TVs in terms of internet capability, such network capability does not seem to completely define a smart TV. In addition to network connection or internet capability, the term "smart TV" would be more appropriate when they include more advanced functions (e.g., advanced user interface, intelligent recommendation for users, and platform for user-created functions) to fit the definition of "smartness". In this sense, smart TVs may be categorized as a subset within the larger category of connected TVs. This report does not discuss specific features or technical details, but is focused on the market and factors relevant to energy consumption in the TVs.

\subsubsection{Smart 'TV (Connected TV) Market}

The success of smart TV relies on dynamic applications, user interface technology, and a high-performance platform rather than on the screen technology itself. Google and Apple, which are leading the markets for internet service and mobile content and devices, are expected to have significant influence on the connected TV market. In addition, personal computer hardware manufacturers are interested in this new market because smart TV must have highly advanced signal processing performance. According to DisplaySearch (2011e), the connected TV market share will increase from 44 million units in 2010 to 123 million units in 2014 when it will account for about $42.6 \%$ of the total TV market, as outlined in Table 3-6.

\begin{tabular}{l|r|r|r|r|r|r}
\hline & \multicolumn{1}{|c|}{2009} & \multicolumn{1}{c|}{2010} & \multicolumn{1}{c|}{2011} & \multicolumn{1}{c}{2012} & \multicolumn{1}{c}{2013} & \multicolumn{1}{c}{2014} \\
\hline connected TV (millions) & 15.2 & 43.9 & 64.4 & 87.2 & 104.8 & 122.7 \\
\hline connected TV share & $7.2 \%$ & $18.0 \%$ & $25.3 \%$ & $33.1 \%$ & $38.1 \%$ & $42.6 \%$ \\
\hline Total TVs (millions) & 210.8 & 243.5 & 254.2 & 263.3 & 275.2 & 287.8 \\
\hline
\end{tabular}

Source: DisplaySearch 2011e $\mathrm{e}^{30}$

\section{Table 3-6. Connected TV Market Forecast}

\subsubsection{Smart TV (Connected 'TV) Energy Consumption}

Smart TVs are expected to consume more energy relative to current conventional (non-smart) TVs because of the following factors: advanced signal processing for additional network connectivity, the potential larger/wider screens and increased daily usage, a default white background screen, and network standby mode.

\section{A] High Performance Components}

Smart TVs require a high-performance central processing unit (CPU), graphical processing unit (GPU), and memory chips for advanced performance and additional network connectivity, as shown in Table 3-7. As a result, according to one manufacturer, a smart TV consumes $10 \%$ more power in its drive circuit than a conventional TV does. Total power consumption for a smart TV is likely to be a few watts higher than for a conventional TV. However, the power increase attributable to these high-performance components can be reduced by employing low-power System-on-Chip (SoC).

\footnotetext{
30 According to DisplaySearch (2011e), the forecast covers TVs with an external connection to a network or directly to the internet (typically through an Ethernet port). Increasing numbers of TV sets employ 802.11n USB dongle, or have wireless networking built in. It is expected that the Ethernet capability in HDMI 1.4 will be adopted, which allows manufacturers to save socket costs.
} 


\begin{tabular}{l|l|l}
\hline & \multicolumn{1}{|c|}{ Conventional TV } & \multicolumn{1}{c}{ Smart TV } \\
\hline CPU & 400 megahertz $(\mathrm{Mhz})$ & 1 gigahertz $(\mathrm{Ghz})$ \\
\hline GPU & 3 million polygons ${ }^{31}$ per second & 30 million polygons per second \\
\hline Memory & 1 gigabyte $(\mathrm{GB})$ & 2GB \\
\hline
\end{tabular}

* Specification can vary with manufacturers.

Table 3-7. Example of Specifications for Image Processing Circuitry in Smart TV versus Conventional TV

\section{B] Wide Screen and High Definition}

It is possible that smart TVs will encourage development of larger and wider screen sizes because consumers may want to do multiple activities through one screen. In other words, "smart" screens may require extra screen space, which in turn would require higher horizontal resolution for multi-view, which can increase power consumption. Figure 3-3 shows possible changes in Smart TV size.

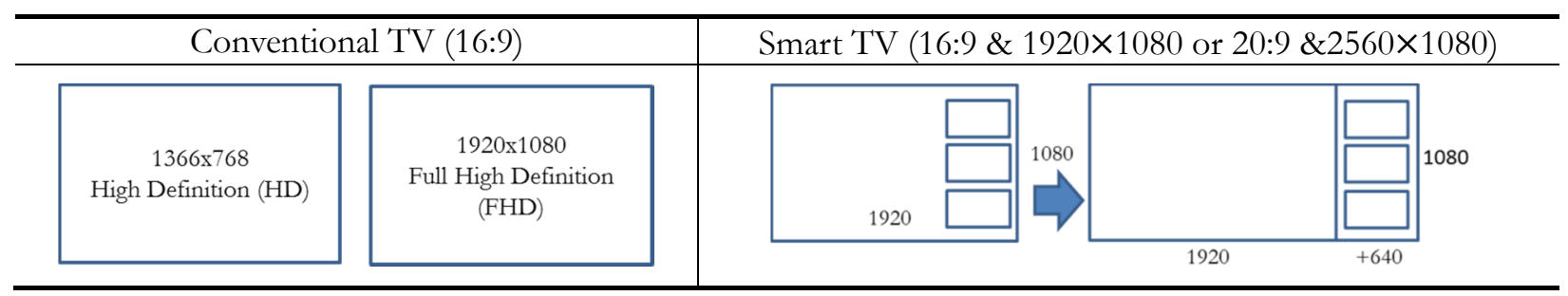

Figure 3-3. Example of Possible Smart TV Size Changes

\section{C] Increased Daily Usage}

It is possible that changes in operating/viewing hours will have a larger effect on energy consumption than unit power consumption increases from advanced hardware specifications. Although the increased range of activities available to users through smart TVs is likely to increase consumers' TV usage/ viewing time, the overlap of such activities with activities like browsing the internet on a separate device such as a laptop or desktop computer is not clear. Therefore, the effect of the development of smart TVs on overall household or global energy consumption is uncertain and needs further study.

\section{D] White Background Default Screen}

Another important factor affecting smart TV energy consumption is the effect of the white-background default screen and the white backgrounds featured on most popular websites. White backgrounds will tend to negate or reduce the effect of local dimming technology in LCD TVs. Although the power consumption of CCFL backlit screens is less sensitive to background color because of the constant backlight in many of these screens, the power consumption of LED backlit LCD screens with dimming options can be affected by changes in background color. In recent exhibitions, some connected TVs introduced by major TV brands and internet/personal-computer-related companies employed black backgrounds for the default page instead of white. On the other hand, the impacts of a black background on power consumption and viewer eye fatigue need further research.

\section{E] Standby Power}

Although a majority of TVs currently consume close to or less than $1 \mathrm{~W}$ in standby mode (or sleep mode), smart TVs (or connected TVs) are, because of network connectivity and integrated features, likely to

31 Polygons (usually triangles) are used in computer graphics to compose 3D images. 
consume more energy in standby mode than conventional TVs. One reason is that connected TVs can be required to rapidly wake from standby mode. Connected TV standby power consumption is estimated at $3 \mathrm{~W}$ to $30 \mathrm{~W}$ although the minimum power requirement for connected TV basic network processing depends on the TV's internal design scheme and specifications and therefore varies among models from different manufacturers. Connected TVs are expected to employ power management regimes, however. For example, it is possible to turn connected TVs from on-mode to a low-power state after a certain period during which the user does not interact with the TV. Idle modes, such as "fast play" and "quick start", can contribute an average of $25 \mathrm{~W}$ to standby power consumption through a user selected option. Although these are not the only network-related features smart TVs (connected TVs) may drive up power consumption through such modes as well as additional network connectivity. This report briefly discusses standby mode power issues in Chapter 6, Other Issues Related to Power Consumption and Efficiency. 


\section{TV Efficiency Improvement Potentials}

In this chapter we review technologically feasible options for TV energy-efficiency improvements that are practical to manufacture, with a focus on LCD, PDP, and OLED TVs.

Because luminance is directly correlated with power consumption as well as with perceived picture quality, it is necessary to consider efficiency improvement options (or power consumption reduction options) at a fixed level of luminance. TV manufacturers control the default luminance level of TVs, but TV panels are usually produced with a certain target luminance level, which varies with type of TV. ${ }^{32}$ The brightness level appropriate for consumers is also relevant to the topic of efficiency improvement, but a full discussion of this issue is beyond the scope of this analysis.

Efficiency in panels is often discussed as a change in luminance $\left(\mathrm{cd} / \mathrm{m}^{2}\right)$ as light flows through each key component of the device (frequently expressed in a percentage). Another important metric used often in discussions of both TV set and panel efficiency, particularly when emphasizing their nature as light sources, is that of luminous efficacy (lumens per W, or cd per ampere). This is the case particularly for LCD backlight, PDP, and OLED panels.

To identify efficiency improvement options for each screen technology, it is necessary to understand different physical principles underlying each technology. Power consumption of self-emissive displays such as PDP and OLED varies with the TV signal, i.e., average picture level (APL) ${ }^{33}$ while LCD TVs can control backlight lamps according to the image signal, to varying degrees depending on the type of dimming technology. This analysis is not intended to compare efficiency of display technologies but to identify technically feasible and manufacturable efficiency improvement options and corresponding incremental costs for each display. The analysis focuses mainly on two kinds of currently available TV displays, LCD and PDP TVs, and one emerging technology, OLED TVs.

Manufacturers are developing efficiency improvement options for LCD and PDP TVs to meet new standards such as ENERGY STAR Version 5 and the European Union (EU) energy labeling program. For example, major TV brands are anticipated to adopt various efficiency improvement options to reduce their on-mode power consumption by up to 30\% and thereby meet ENERGY STAR Version 5 requirements. In this section we discuss these options and their relevance in the context of a market transformation program to improve energy efficiency.

\subsection{LCD T'Vs}

In this subsection we discuss key factors affecting efficiency improvement in LCD TVs, technology options to improve efficiency in LCD TVs, and corresponding estimated costs where cost data are available.

\subsubsection{Key Factors Related to Efficiency Improvement in LCD TVs}

When considering the efficiency of LCD TVs conceptually, it is useful to divide TV sets into the following main parts: the BLU, the LCD panel, and the rest of the TV set.

\footnotetext{
32 In general, although LCD TV panels over 30 inches have been manufactured with a target luminance of 400 to $500 \mathrm{~cd} / \mathrm{m}^{2}, 42-i n c h$ HD PDP panels without filters have been produced with a target luminance of 160 to $200 \mathrm{~cd} / \mathrm{m}^{2}$ at full-white mode (APL $100 \%$ ). Luminance in PDP panels varies with APL from 180 to $200 \mathrm{~cd} / \mathrm{m}^{2}$ in full-white mode to 1,000 to $1,300 \mathrm{~cd} / \mathrm{m}^{2}$ at $1 \%$ peak window. In normal video mode, corresponding to APL 20\% to 40\% , PDP panels are about twice as bright as they are in full-white mode, and the final luminance of a PDP TV becomes about half of that of its panel because of a filter with $40 \%$ to $48 \%$ transmittance.

33 There are two definitions of APL: One (Pre-Gamma) is the time average of a video signal input voltage to a TV set, which is usually expressed as a percentage of the full $(100 \%)$ white signal level voltage. The other (Post-Gamma) is the time average of the average luminance of all pixels in the TV set, which is usually expressed as a percentage of the peak white luminance level (Fraunhofer 2007a).
} 
Although LCD TVs are much brighter than PDP and CRT TVs, LCD TVs' overall efficiency is not greater in terms of change in luminance $(\mathrm{cd} / \mathrm{m} 2)$ as light travels through the LCD TV set. Assuming that the initial luminance from the backlight unit is $100 \%$, the final luminance is about 4 to $6 \%$ of this initial luminance, largely because of the low transmittance of the LCD panel. If panel transmittance and efficiency of optical films improve, the backlight will not be required to produce such high luminance. Such improvements would enable reduction of the number of backlight lamps, lowering power consumption. According to a manufacturer, the efficiency of the PSU is now between $85 \%$ and $95 \%$. Thus, efficiency improvement in LCD TVs will largely depend on improvements in the BLU and LCD panel. Table 4-1 shows the change in luminance for an LCD TV.

\begin{tabular}{|c|c|c|}
\hline & Key Components & Luminance \\
\hline $\operatorname{Set}^{34}$ & $\begin{array}{l}\text { Digital Signal Processing } \\
\text { Power Supply Unit }\end{array}$ & $\begin{array}{r}380-450 \mathrm{~cd} / \mathrm{m}^{2} \\
(3.8-5.6 \%)\end{array}$ \\
\hline Panel $^{35}$ & $\begin{array}{l}\text { Polarizers } \\
\text { Color Filter } \\
\text { Liquid Crystal } \\
\text { Thin Film Transistor array } \\
\text { Drive integrated circuits }\end{array}$ & $\begin{array}{r}450-500 \mathrm{~cd} / \mathrm{m}^{2} \\
(4.5-6.3 \%)\end{array}$ \\
\hline BLU & $\begin{array}{l}\text { Optical Films } \\
\text { Diffuser Plate / Light Guide Panel } \\
\text { Light Source }\end{array}$ & $\begin{array}{r}8,000-10,000 \mathrm{~cd} / \mathrm{m}^{2} \\
(100 \%)\end{array}$ \\
\hline
\end{tabular}

* The final luminance of the BLU is regarded as the starting point, $100 \%$.

Therefore, this does not capture the luminous efficiency of the BLU itself.

Table 4-1. Example of Change in Luminance for a Typical LCD TV Set

In this analysis we review technical opportunities for efficiency improvement in LCD TVs in the following areas, which are also areas in which manufacturers have been focusing product development efforts:

1) BLU: efficient backlight source and improved structure

2) BLU: efficient optical films

3) Panel: increased transmittance

4) Power management at on-mode: dimming technology

Options 1) and 4) can directly reduce LCD TV power consumption while options 2) and 3) help reduce power consumption indirectly by reducing the number of backlight lamps necessary to achieve the same luminance level. Option 3) can reduce the power needed to drive the LCD panel.

\subsubsection{Technology Options for Efficiency Improvement of LCD T'Vs}

In this subsection we discuss various options to improve the efficiency of LCD TVs, including different backlight sources (CCFL and LED), changes in backlight structure, improvement in backlight source efficiency, improvement in BLU efficiency by using various optical films, improvement in panel transmittance, and improvement in power management.

\footnotetext{
34 According to manufacturers, the luminance level of LCD TV sets is $5 \%$ to $10 \%$ lower than that of LCD panels because of electrical losses in the circuitry and power supply.

35 Panel transmittance varies among different TV models and manufacturers. According to manufacturers, 100/120 Hz-driven LCD panels have 5\% to $6 \%$ transmittance on average, and 200/240 Hz-driven LCD panels, including 3D LCDs, have 4\% to 5\% transmittance on average. In general, manufacturers compensate for lower transmittance by using more efficient optical films or adjusting the backlight structure by changing the target luminance level of the BLU.
} 


\section{A] Backlight Source Type: CCFL and LED}

We screened out LED backlit LCD TV models and CCFL backlit LCD TVs from ENERGY STAR Version 4 TV list (ENERGY STAR 2010) according to major manufacturers' catalogs and websites. Based on the selected data, LED-edge backlit LCD TVs are 20\% to 30\% more efficient in on-mode power consumption than CCFL backlit LCD TVs. Figure 4-1 shows the average W/in ${ }^{2}$ of LCD TVs by backlight type.

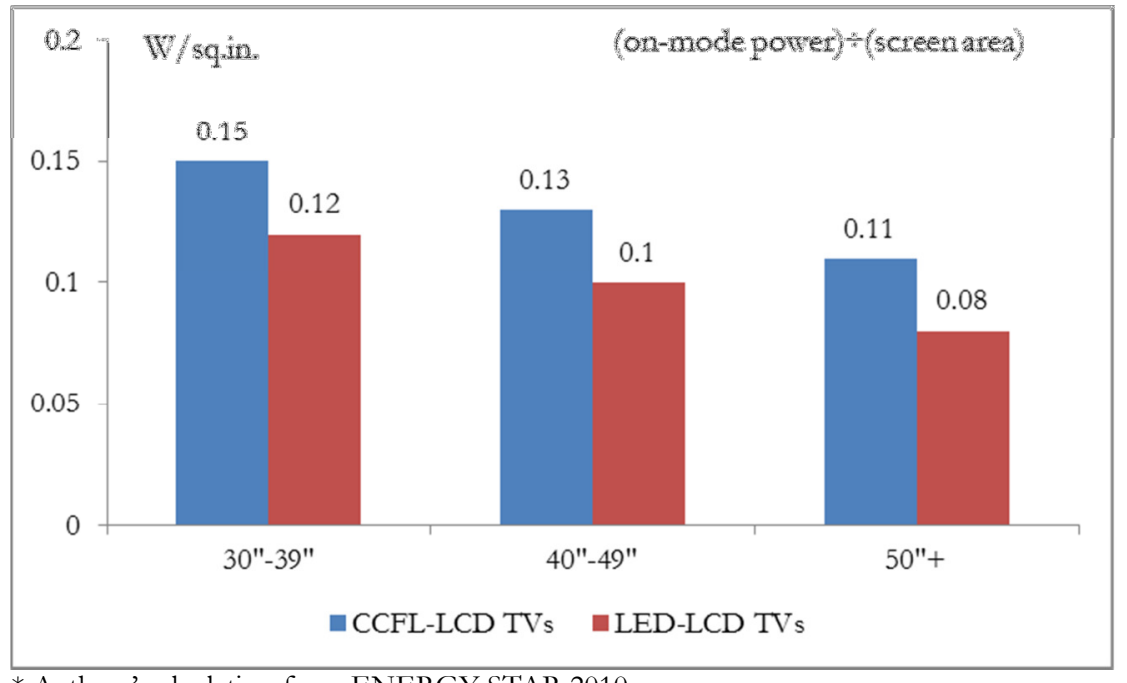

* Authors' calculation from ENERGY STAR 2010

\section{Figure 4-1. Average Watts per Square Inch of LCD TVs by Backlight Type}

However, the average retail price of LED backlit TVs is higher than that of CCFL backlit TVs. In particular, LCD module price accounts for a large portion of total TV set production cost, and the difference between set production cost and module price for both types is similar, in the range of $\$ 120$ to $\$ 135$. Backlight type affects both total manufacturing cost and energy consumption. Figure 4-2 shows the average U.S. retail price and manufacturing cost for 32-inch LCD TVs.

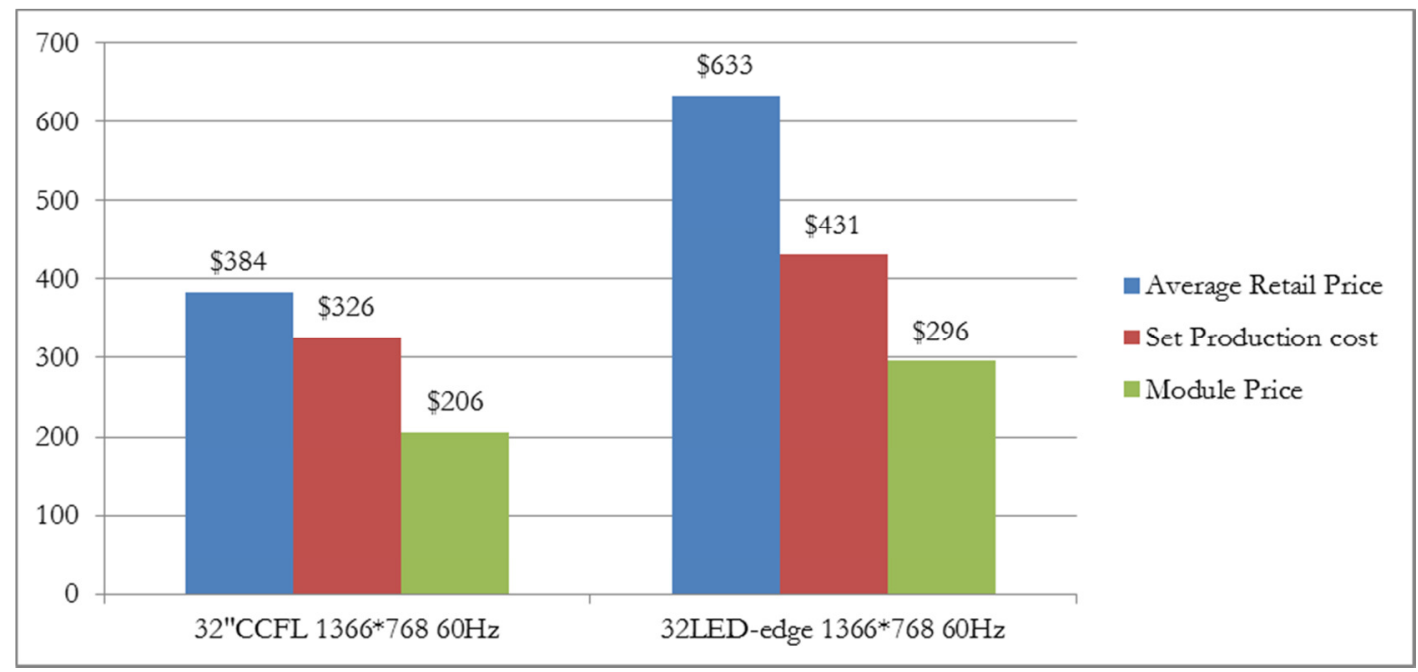

Source: DisplaySearch 2010d, 2010f

Figure 4-2. Average U.S. Retail Price and Manufacturing Cost for 32-inch LCD TVs (Q2 2010) ${ }^{36}$

\footnotetext{
36 Set Production Cost $=$ LCD module price + mechanical/electronic parts + packaging $/$ accessories + royalties +
} 
Materials and components weighted by production yield account for about $70 \%$ to $80 \%$ of the total manufacturing cost of LCD TV panels. In the case of 32-inch LCD TV panels with 1,366×768 resolution, the difference between total manufacturing cost and material/component cost is the same regardless of backlight type, indicating that components other than the BLU are largely the same, and the cost of the LED BLU is 3.4 times that of the CCFL backlight. Figure 4-3 shows LCD module manufacturing costs for 32-inch LCD TVs.

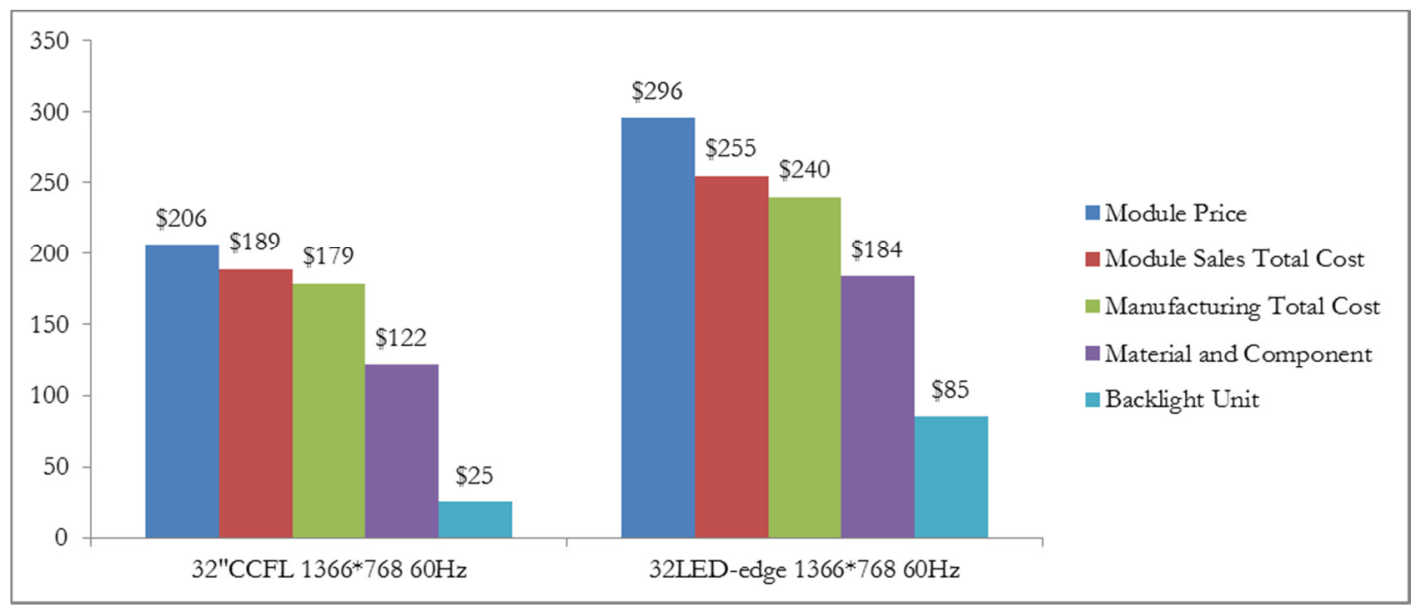

Source: DisplaySearch 2010d

\section{Figure 4-3. LCD Module Manufacturing Costs for 32-inch LCD TVs (Q2 2010) ${ }^{37}$}

To accelerate market penetration of high-efficiency TVs, one option might be to encourage a rapid transition from CCFL to LED backlights. Although the average market price of LED backlit TVs is currently about 1.4 times that of CCFL backlit TVs, it is expected that the gap will narrow to about 1.2 in 2012. Figure 4-4 graphs the price gaps between CCFL and LED backlit TVs.

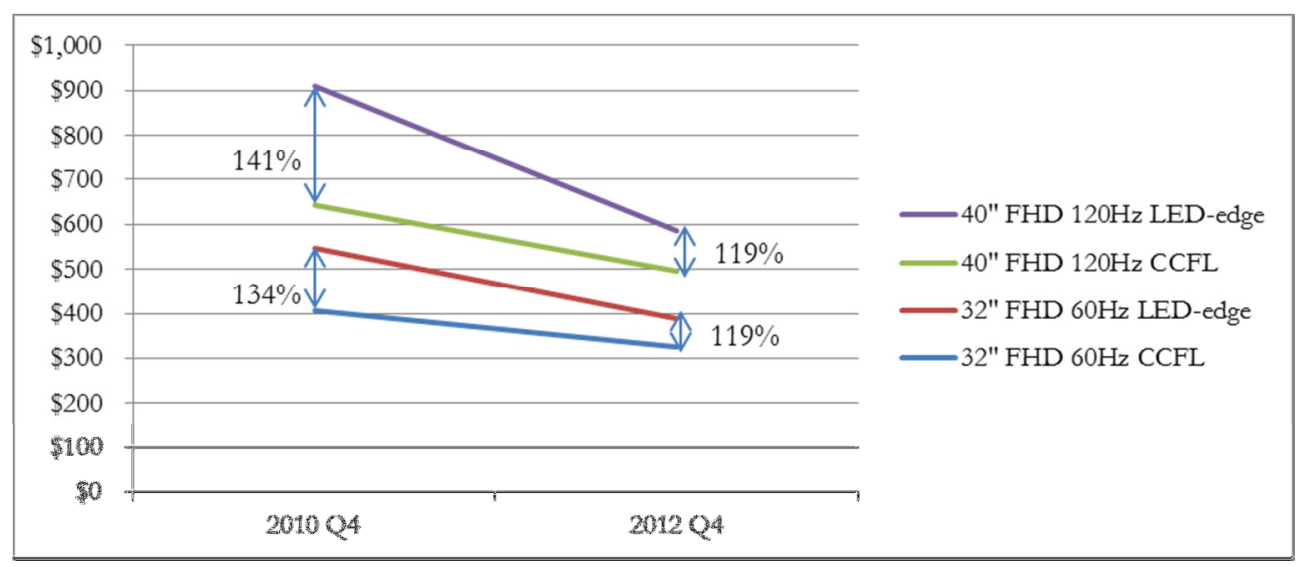

Source: DisplaySearch $2010 \mathrm{f}$

Figure 4-4. Price Gaps between CCFL backlit TVs and LED backlit TVs

As mentioned in Section 2.3.2, major TV brands are expected to provide more LED backlit TV models at lower prices in emerging markets. Lowering the maximum luminance level and color-reproduction capability

labor/overhead/profit (calculated by author). In general, it takes 1 month to assemble LCD TV sets from LCD modules, so the module price from the previous quarter is applied to the current TV set production cost and market price.

$3784.9 \%$ yield is applied to the material and component cost. 
allows manufacturers to reduce BLU materials costs. This suggests that the barrier to further market penetration of LED backlit TVs is mainly cost.

\section{B] Backlight Structure: LED-direct (full array) and LED-edge}

Figure 4-5 shows the conceptual structure of typical backlights. As mentioned earlier, LED-edge backlit TVs are expected to dominate the market because they offer more benefits for manufacturers and consumers in terms of cost, design, and energy consumption. LED-direct TV local dimming technology is more effective in reducing power than global dimming or partial dimming, but LED-edge TVs have an advantage over LED-direct TVs in resource use and manufacturing cost.

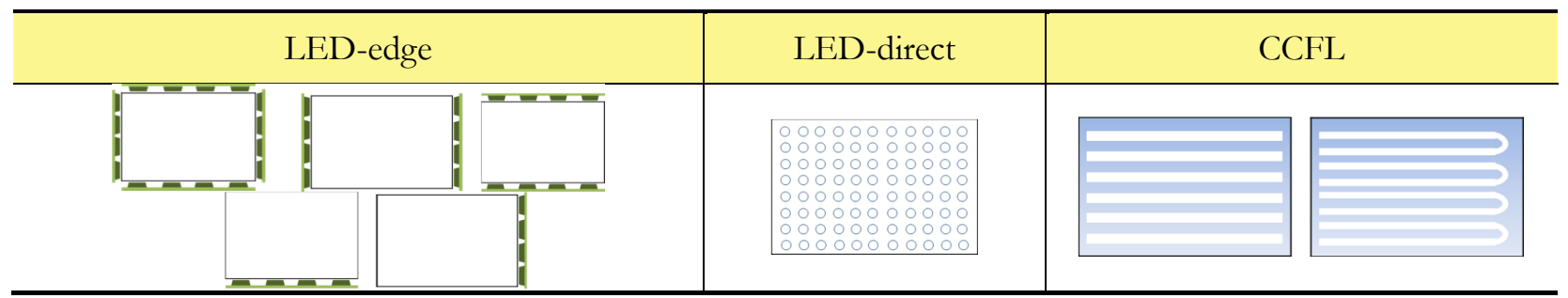

Figure 4-5. Conceptual Structure of Typical Backlights

Table 4-2 shows a comparison of 46-inch LCD TVs by backlight type. LED-edge backlighting usually consumes less power than LED-direct backlighting at maximum brightness level, while local dimming of LED-direct backlights is more effective than complete or line dimming of LED-edge backlights in power reduction at on-mode. Total impacts of LED BLUs on power consumption vary with LED efficacy, input power, number of LEDs used, and dimming technology. In addition, the beam angle of LEDs is a limiting factor for designing LED BLUs. According to an expert, even though LED-direct backlighting with widebeam-angle LEDs can, without dimming options, achieve a power consumption similar to that of LED-edge backlighting, the manufacturing cost of LED-direct TVs is higher than that of LED-edge TVs. Table 4-3 compares the technical specifications for 32-inch LED-edge and LED-direct TVs, including numbers and costs of LEDs. LED-direct backlit TVs requiring hundreds of LEDs are typically employing lower-power $(<0.2 \mathrm{~W})$ LEDs compared to LED-edge backlit TVs.

\begin{tabular}{|c|c|c|c|}
\hline Backlight Type & LED-edge & LED-direct & $\mathrm{CCFL}$ \\
\hline Thickness (mm: millimeters) & $10.8 \mathrm{~mm}$ & $46 \mathrm{~mm}$ & $32.5 \mathrm{~mm}$ \\
\hline Weight (kg: kilograms) & $11 \mathrm{~kg}$ & $17.5 \mathrm{~kg}$ & $12.3 \mathrm{~kg}$ \\
\hline Power Consumption ${ }^{38}$ & $126 \mathrm{~W}$ & $175 \mathrm{~W}$ & $210 \mathrm{~W}$ \\
\hline Contrast Ratio $^{39}$ & 10,000:1 & 100,000:1 & $10,000: 1$ \\
\hline Number of Lamps & 324 (LED) & 640 (LED) & 16 (CCFL) \\
\hline Manufacturing Cost & $100 \%$ & $120 \%$ & $60 \%$ \\
\hline
\end{tabular}

Source: Jang 2009

Table 4-2. Comparison of 46-inch (904-cm) LCD TVs by Backlight Type

\footnotetext{
38 These are not on-mode power data but power consumption in full white mode without dimming options.
}

39 Complete dimming is applied to LED-edge and CCFL, and local dimming to LED-direct. 


\begin{tabular}{l|l|l}
\hline Backlight Type & LED-edge & LED-direct \\
\hline $\mathrm{I}_{\mathrm{f}}{ }^{40}$ & $90-100 \mathrm{~mA}$ & $60-65 \mathrm{~mA}$ \\
\hline $\mathrm{V}_{\mathrm{f}}$ & $3.0-3.2 \mathrm{~V}$ & $3.0-3.2 \mathrm{~V}$ \\
\hline $\mathrm{P}_{\mathrm{LED}}\left(\mathrm{I}_{\mathrm{f}} * \mathrm{~V}_{\mathrm{f}}\right)$ & $0.27-0.32 \mathrm{~W}$ & $0.18-0.21 \mathrm{~W}$ \\
\hline Number of LEDs & $80-10041$ & $280-30042$ \\
\hline Cost per LED & $\$ 0.2-\$ 0.23$ & $\$ 0.14-\$ 0.18$ \\
\hline
\end{tabular}

If: forward current; Vf: forward voltage; mA: milli-ampere ; V: volts

Source: manufacturer and expert interviews

\section{Table 4-3. LED Backlight Specifications for 32-inch $(81.3-\mathrm{cm})$ LCD TVs}

\section{C] Backlight Source Efficiency Improvement: High-Efficiency LEDs}

As the luminous efficacy (lumens per watt $[\mathrm{lm} / \mathrm{W}]$ ) improves, the energy efficiency of LEDs used for LCD backlit units will improve. According to the U.S. Department of Energy (DOE), during the next few years LED light sources will surpass the performance capability of incandescent and fluorescent lighting, and by 2015 LED luminaires will be capable of producing an efficacy of more than $150 \mathrm{~lm} / \mathrm{W}$ (DOE 2010). Figure 4-6 shows forecast luminous efficacy for LEDs.
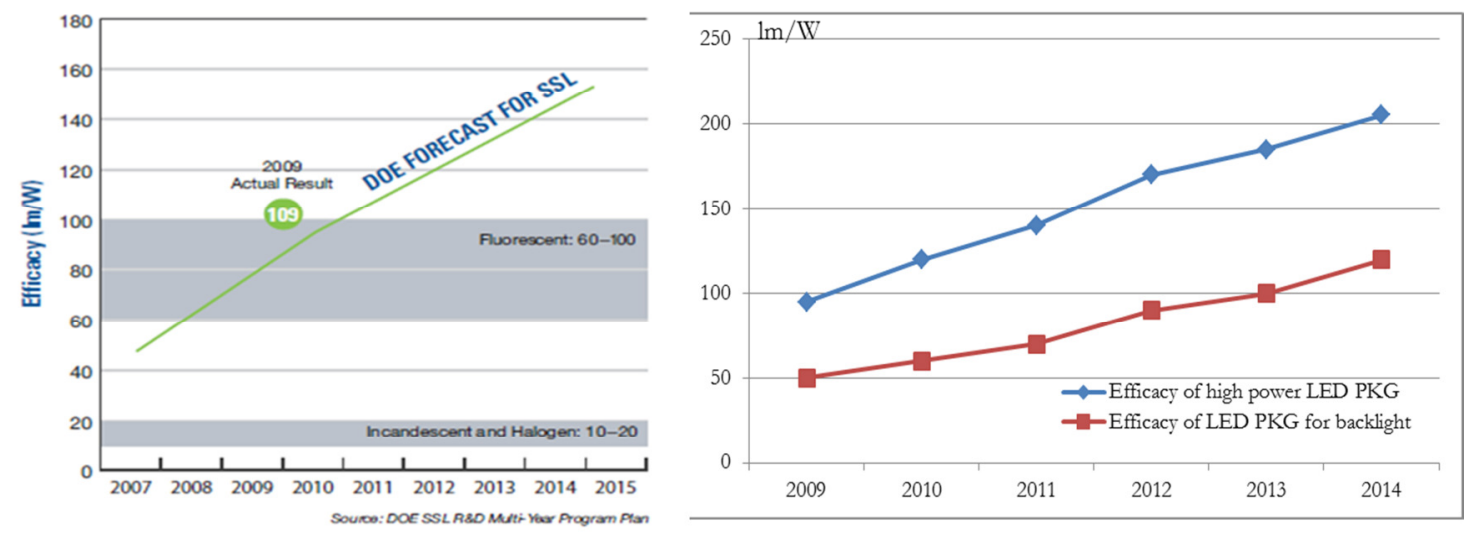

Sources: (left) DOE 2010a; (right) LED manufacturer

Figure 4-6. LED Roadmap for Luminous Efficacy $(\mathrm{lm} / \mathrm{W})$

According to experts, existing LED backlit TVs on the market use $60 \mathrm{~lm} / \mathrm{W}$ LEDs on average; LEDs of 80 to $100 \mathrm{~lm} / \mathrm{W}$ are expected to be used for LCD backlighting in the next 2 to 3 years. By using more efficient LEDs, TV manufacturers can reduce the minimum number of LED lamps necessary for LCD backlit units. However, it is difficult for manufacturers to immediately employ existing higher-efficiency LEDs that are being used for general illumination. High-efficiency LEDs operate at higher power levels ( $>1 \mathrm{~W})$ than LEDs used in TVs $(\sim 0.4 \mathrm{~W})$. High-power LEDs generate more heat than mid-power LEDs, so TV manufacturers

\footnotetext{
$40 \mathrm{I}_{\mathrm{f}}$ - forward current, i.e., the electrical current flowing through a semi-conductor diode. In general, a small change in forward voltage $\left(\mathrm{V}_{\mathrm{f}}\right)$, which is the voltage across a semi-conductor diode that carries current in the forward direction, produces a disproportionately large change in forward current.

41 In case LEDs are located on one horizontal side (bottom), or two vertical sides (left/right).

42 Sharp LE700UN models have adopted LED-direct backlight units and consume less power than other LED-direct type TVs because the model uses fewer LEDs with wide-beam angle than other LED-direct TVs.
} 
would need to develop better thermal management techniques to incorporate high-efficiency and high-power LEDs to TVs. Therefore, improving the efficiency of LEDs used in TVs will require development of highefficiency LEDs at mid-power or advanced thermal management systems for high-efficiency and high-power LEDs.

\section{a. Technology Options for Development of High-Efficiency LEDs}

- LED Structure: multiple quantum well (MQW) structure

- Phosphors: silicon dioxide $\left(\mathrm{SiO}_{2}\right)$-based powder to silicon nitride $\left.\mathrm{SiN}_{2}\right)$-based powder

- Thermal Management: improved packaging technology

- LED Performance: wide-beam-angle LEDs

For development of high-efficiency LEDs, improvement in LED structure is a key possibility. As electrical current in a single quantum well ${ }^{43}$ structure increases, optical output increases in a logarithmic scale. A multiple quantum well structure has more than one well that can accommodate more carriers than a single quantum well, resulting in more optical output. Figure 4-7 shows an example of LED structure with MQWs.

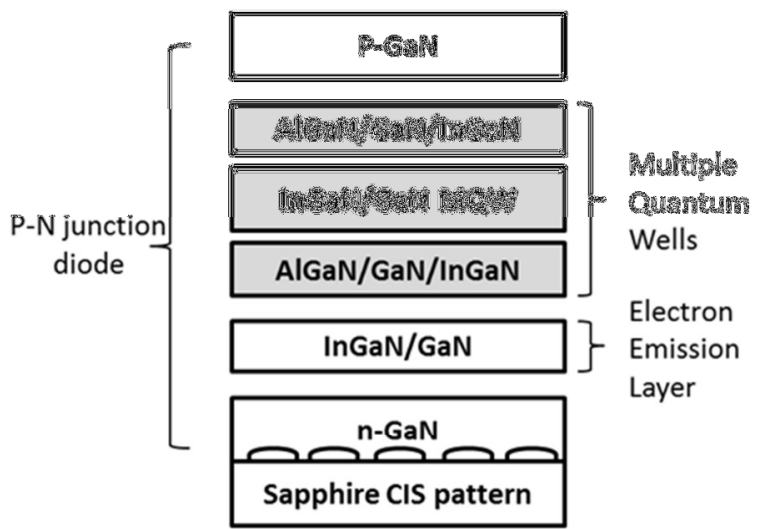

Light Output Characteristics

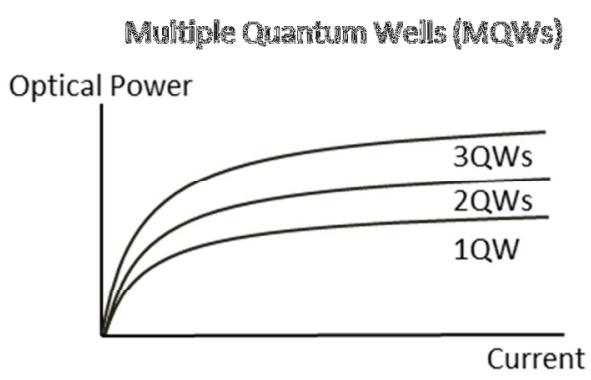

Source: manufacturer

Figure 4-7. Example of LED Structure with Multiple Quantum Wells (MQWs)

Second, development of new phosphors is an important efficiency improvement. However, in general, the luminous efficacy of a phosphor is traded off against color gamut ${ }^{44}$. For example, while B+RG (Blue LED+Red/Green phosphors) can produce a wider color gamut $83 \%$ at National Television System Committee [NTSC]) than B+Y (Blue LED+Yellow phosphor) (68\% at NTSC), the former consumes more power. In general, the color gamut of an LCD TV is determined by color filters and backlight lamps. The color gamut of normal CCFL backlit TVs is $72 \%$ and that of advanced CCFL backlit TVs is more than $85 \%$. Although white LEDs with B+Y (Blue LED+Yellow phosphor) are more efficient than other types of white LEDs, they are not acceptable for monitors or TVs. White LEDs with B+RG (blue LED + Red/Green phosphors) are being widely used for TV applications. According to experts, the existing $\mathrm{SiO}_{2}$-based phosphors can be replaced with $\mathrm{SiN}_{2}$-based phosphors, and the protective layer on the phosphors can be improved, resulting in high efficiency. According to a manufacturer, Table 4-4 compares the efficiency and color gamut of different phosphors.

\footnotetext{
43 A quantum well means a region surrounding a minimum of potential energy with discrete energy values.

44 Color gamut is a certain complete set of colors, commonly represented as areas in the CIE 1931 Chromaticity Diagram or in National Television System Committee (NTSC) 1987.
} 


\begin{tabular}{l|c|c|c}
\hline & $\begin{array}{l}\text { Blue LED } \\
+ \text { Yellow Phosphor }\end{array}$ & $\begin{array}{l}\text { Blue LED } \\
+ \text { Green/Orange Phosphors }\end{array}$ & $\begin{array}{l}\text { Blue LED } \\
+ \text { Red/Green Phosphors }\end{array}$ \\
\hline Efficiency & Better & Good & Fair \\
\hline $\begin{array}{l}\text { Color Gamut } \\
\text { (NTSC) }\end{array}$ & $68 \%$ & $70 \%$ & $83 \%$ \\
\hline
\end{tabular}

Source: manufacturer

\section{Table 4-4. Color Gamut and Efficiency of Phosphors}

Third, packaging technology is important to maximize lifetime and efficiency. The molding compound needs to be durable at high temperature and highly reflective. For better thermal management, the lead frame needs to contact the printed circuit board directly.

Lastly, LEDs with wider beam angles (greater than 120 degrees) can help manufacturers reduce the number of LEDs, compared with conventional LEDs with 60- to 120-degree beam angles. In general, wide-beamangle LEDs are being used in LED-direct BLUs. Some experts claim that there are optical benefits to beaming the light straight forward in edge lit designs, while some others say that straight-beaming LEDs may allow LCD panels to have "dark spots" between LED chips as the number of LEDs decreases in edge lit structure as well as a full-array (direct) structure. Usefulness of wide-beam angle LEDs in edge lit designs needs further research.

In the 2010 TV market, various structures were used for LED backlighting. In the most efficient case, LED backlight bars are located on one horizontal side (bottom) or two vertical sides (left/right). This design choice depends primarily on manufacturers' strategies and technical preferences. According to experts, those two types are expected to dominate in the short term for screen sizes greater than 30 inches $(76.2 \mathrm{~cm})$. Table 4-5 traces expected short-term improvements for both types of LED placements.

Type 1: One Horizontal Side

\begin{tabular}{|c|c|c|c|}
\hline Year & 2010 & 2011 & 2012 \\
\hline Backlight type & LED-edge & LED-edge & LED-edge \\
\hline \multicolumn{4}{|l|}{ Structure } \\
\hline LED Spec. & $60 \mathrm{~lm} / \mathrm{W}$ & $70 \mathrm{~lm} / \mathrm{W}$ & $80-85 \mathrm{~lm} / \mathrm{W}$ \\
\hline LED Power & $0.32 \mathrm{~W}$ & $0.38 \mathrm{~W}$ & $0.43 \mathrm{~W}$ \\
\hline Number of LEDs used & $100 \%$ & $80 \%$ & $70 \%$ \\
\hline Power (BLU only) & $100 \%$ & $95 \%$ & $94 \%$ \\
\hline \multicolumn{4}{|c|}{ Type 2: Two Vertical Sides } \\
\hline Year & 2010 & 2011 & 2012 \\
\hline Backlight type & LED-edge & LED-edge & LED-edge \\
\hline \multicolumn{4}{|l|}{ Efficient Structure } \\
\hline LED Spec. & $60 \mathrm{~lm} / \mathrm{W}$ & $70 \mathrm{~lm} / \mathrm{W}$ & $80-85 \operatorname{lm} / \mathrm{W}$ \\
\hline LED Power & $0.32 \mathrm{~W}$ & $0.38 \mathrm{~W}$ & $0.43 \mathrm{~W}$ \\
\hline Number of LEDs used & $100 \%$ & $75 \%$ & $63 \%$ \\
\hline Power (BLU only) & $100 \%$ & $89 \%$ & $84 \%$ \\
\hline
\end{tabular}

Table 4-5. Effect of LED Improvement on Power Consumption, 32-inch LCD TVs

Even as LEDs with higher luminous efficacy are employed in TVs, the ratio of LED power to efficacy will likely increase slightly. Thus, for one-horizontal-side-mount LED-edge TVs, the efficacy is expected to improve $33-42 \%$ from $60 \mathrm{~lm} / \mathrm{W}$ to $80-85 \mathrm{~lm} / \mathrm{W}$, and power consumption will decrease by about $6 \%$. For twovertical-sides-mount LED backlit TVs, if the efficacy improves $33-42 \%$ from $60 \mathrm{~lm} / \mathrm{W}$ to $80-85 \mathrm{~lm} / \mathrm{W}$, power 
consumption will decrease by about $16 \%$. These numbers indicate that improving LED efficacy has a greater impact on efficiency for LED edge backlit TVs with two vertical sides because the reduction in number of LEDs is greater with the same LED efficacy improvement. According to DisplaySearch (2011b), it is expected that one vertical-sided structure will be implemented in some entry-level TV models in the 32- to 37-inch (81.3- to $94.0-\mathrm{cm})$ screen size range.

Moreover, assuming that both types of LED backlighting use same type and quality of LEDs, the current vertical-sided structure is $20 \%$ more efficient than the horizontal-sided structure because the vertical structure requires $20 \%$ fewer LEDs than the horizontal type. However, LED backlight structure is associated with panel transmittance and other optical parts in a BLU, so a choice between two backlight structures depends on manufacturer strategy and technical design preferences.

The current average cost of one LED chip used for LED-edge backlighting is $\$ 0.2-0.24$. This cost is not expected to increase even if efficiency improves. Some experts suggest that the price might decrease as the market volume of LED backlit TVs grows and a new type of LED becomes dominant in TV application. In addition, Haitz's Law indicates that, ${ }^{45}$ as the light output of LEDs increases, the cost (\$ per lumen) tends to decrease. See Figure 4-8.

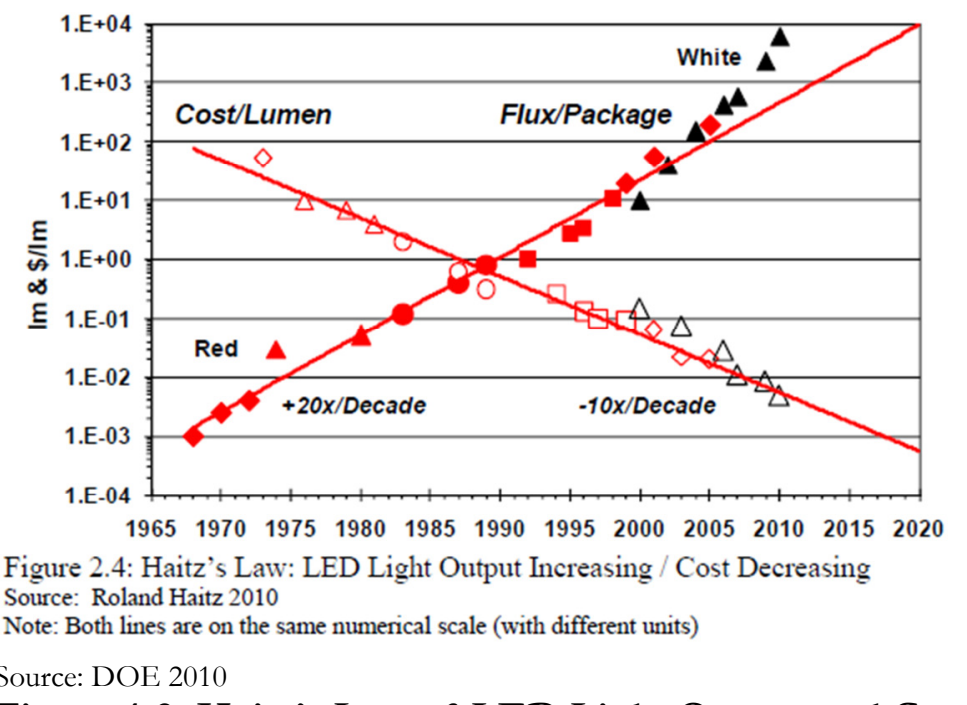

\section{Figure 4-8. Haitz's Law of LED Light Output and Cost}

\section{b. Conclusions Regarding Development of High-Efficiency LEDs}

TV manufacturers can reduce material costs as a result of LED efficiency improvements. Cost reduction is an intrinsic motivation for manufacturers to achieve high efficiency in LED backlights. Therefore, we expect that manufacturers will likely continue to pursue adoption of high-efficiency LEDs even in the absence of a market transformation program. LED efficacy is expected to improve as a result of LED structure, phosphor, heat-solution, and beam angle developments. However, it is likely that the efficacy of LEDs used in TVs will lag the efficacy of LEDs available on the market because of structural issues such as heat dissipation. Reducing this lag is an important option that a market transformation program could pursue to improve the energy efficiency of LED backlit TVs.

Under the following assumptions, the relationship between LED efficiency improvement and costs can be described as shown in Figure 4-9.:

\footnotetext{
45 The light output of LEDs has increased twentyfold each decade for the past 40 years, while the cost (\$/lumen) has decreased tenfold each decade during that same time period (DOE 2010b).
} 
- Except for a few models with one-vertical-side-mount structure, all other LED backlit TV entry models of 30-40 inches will have two types of backlight structure: one-horizontal-side-mount and two-vertical-side-mount.

- As LED efficacy improves, the corresponding reduction in power consumption will not be significant because high-efficiency LEDs are also high-power LEDs. Power reduction will instead be achieved by reducing the number of LEDs used.
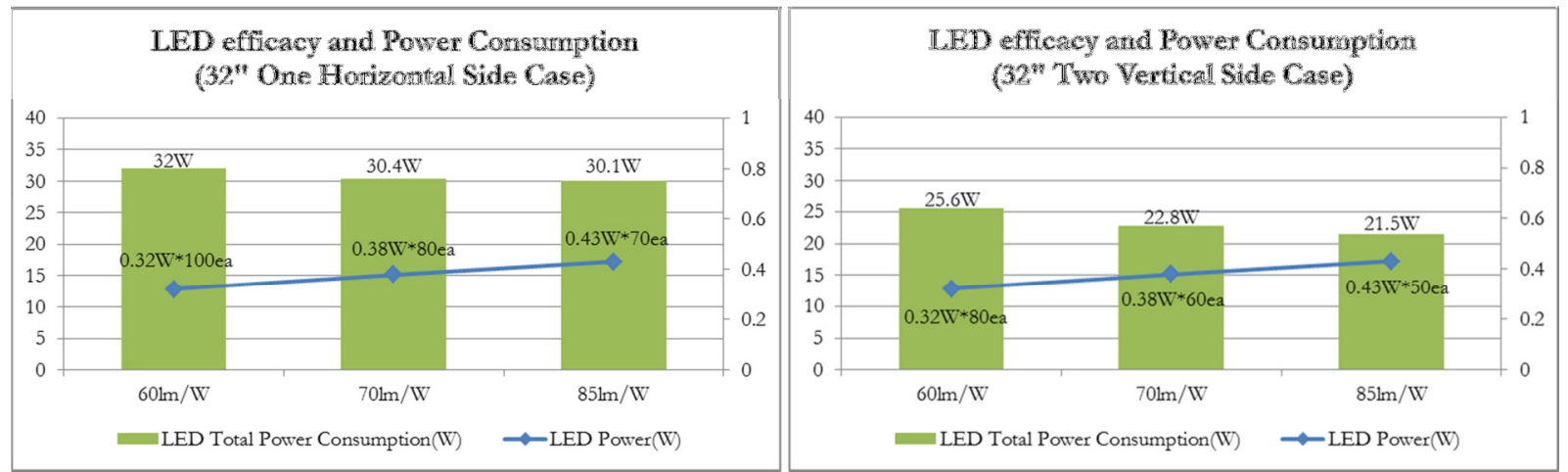

Figure 4-9. LED Efficacy Improvement $(\mathrm{lm} / \mathrm{W})$ and BLU Power Consumption (W)

\section{D] Optical Films}

In addition to the light source itself, the LCD BLU has various optical parts. Although DBEF (a reflective polarizer film manufactured by $3 \mathrm{M}$ ) has the most significant impact among optical films on BLU efficiency, it is expensive and comes from a single manufacturer. For these reasons, manufacturers have been adopting various combinations of films, depending on panel transmittance, light source efficacy, and other specifications. Of these other optical components, reflective polarizers and prism sheets have the most influence on efficiency improvement. Table 4-6 shows the types of optical films used in LCD BLUs.

\begin{tabular}{|c|c|c|c|c|}
\hline Components & \# used & \multicolumn{3}{|c|}{ Structure } \\
\hline & & $-2-2-2$ & ICD Panel & \\
\hline & & 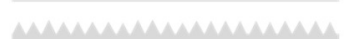 & & \\
\hline Prism sheet(s) & $1-3$ & $\begin{array}{l}\square \\
\square\end{array}$ & $\begin{array}{l}\text { Diffuser } \\
\text { Larmp \& i Light G Guide Pancl }\end{array}$ & $\begin{array}{l}\text { Beckighat Unit } \\
\text { (BLLC) }\end{array}$ \\
\hline Diffuser(s) & $1-3$ & Edge-type BLU & $\begin{array}{l}\text { Reflector } \\
\text { LCD Panel }\end{array}$ & \\
\hline $\begin{array}{l}\text { Light Guide Panel (LGP) or } \\
\text { diffuser plate* }\end{array}$ & 1 & 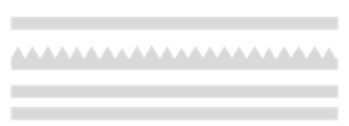 & $\begin{array}{l}\text { Reflective Polarizer } \\
\text { Prism Sheet } \\
\text { Diffisuser } \\
\text { Diffuser Plate }\end{array}$ & $\begin{array}{l}\text { Backighat Unit } \\
\text { BLY }\end{array}$ \\
\hline Reflector & 1 & 000000 & $\begin{array}{l}\text { Lamp } \\
\text { Refecector }\end{array}$ & \\
\hline
\end{tabular}

* LGP is used for an edge-type backlight, and diffuser plate is for direct-type.

\section{Table 4-6. Optical Films used in LCD BLU}

Typically, a reflector increases the light reflected from the rear of the backlight. A light guide panel (LGP), which is used for edge-type backlighting, guides and scatters the light emitted from the source toward the front of the TV. Diffusers distribute the light toward the LCD panel uniformly so that the structures of the LED array and LGP are smoothed out in the light. Prism films are important to optimize the angle of light 
and to direct light toward the LCD panel. Reflective polarizers optimize the light's polarization and minimize the amount of light absorbed by the panel's polarizers (3M 2010a).

\section{a. Prism Films (Lens Films)}

In general, prism films are designed with high transparency and high refractive index to recycle the wasted component of luminous flux from the backlight source. 3M's brightness enhancement film (BEF) is the most common lens film used by display manufacturers. The film is placed on the top of a stack of films. Conventionally, two orthogonally arranged films are used because a sheet of the film can redirect off-angle light from one direction only (horizontal or vertical). There are several types of lens films, including 3M BEF, that can enhance brightness from 20\% to 60\% (Figure 4-10), compared to backlighting without films.
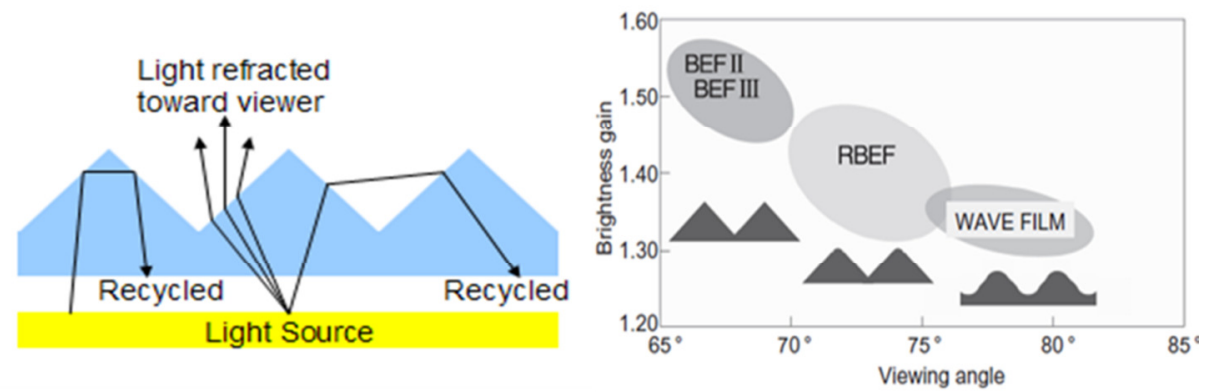

Source: (left) DisplaySearch 2011c, (right) Hanzawa 2009

\section{Figure 4-10. Brightness Enhancement Film (BEF) and Brightness Gain of Various Lens Films}

Typical prism films such as BEF provide better brightness gain with sharp-tipped lenses; prism films with round-tipped lenses give products a wider viewing angle compared to what is possible with sharp-tipped prism films. In addition, this lenticular type of film is easy to manufacture and cost competitive, compared to typical prism films with sharp-tipped lenses.

\section{b. Reflective Polarizers}

A DBEF recovers a certain type of polarized light, ${ }^{46}$ which cannot be transmitted through the rear polarizer of the LCD panel, by reflecting this portion of light back to the BLU and depolarizing it so that the light can be newly polarized to transmit back to the panel. According to 3M, a DBEF can improve energy efficiency by $20 \%$ to $30 \% .{ }^{47}$ Manufacturers have been using DBEF in many products and agree that it is one of the best options for efficiency improvement, but they have also been identifying other combinations of optical films excluding DBEF because DBEF is an expensive, proprietary technology. The patent for DBEF will expire in 2016. Although similar films are provided by Korean and Japanese suppliers, 3M's DBEF is recognized as the best among the existing reflective polarizers. Reflective polarizers have hundreds of layers whose thickness is less than 100 nanometers $(\mathrm{nm})$. Manufacturing reflective polarizers with such thin layers is a key technical barrier to other suppliers being more competitive (DisplaySearch 2011c). Figure 4-11 shows light utilization with and without DBEF.

\footnotetext{
46 While the p-polarized light is transmitted through the LCD rear polarizer into the LCD, the s-polarized light is absorbed at the entrance of the panel.

47 A 37-inch CCFL LCD TV with two diffusers and one DBEF consumed 145 W, and a 37-inch CCFL LCD TV with three diffusers consumed 180 W. (Fraunhofer 2007d)
} 


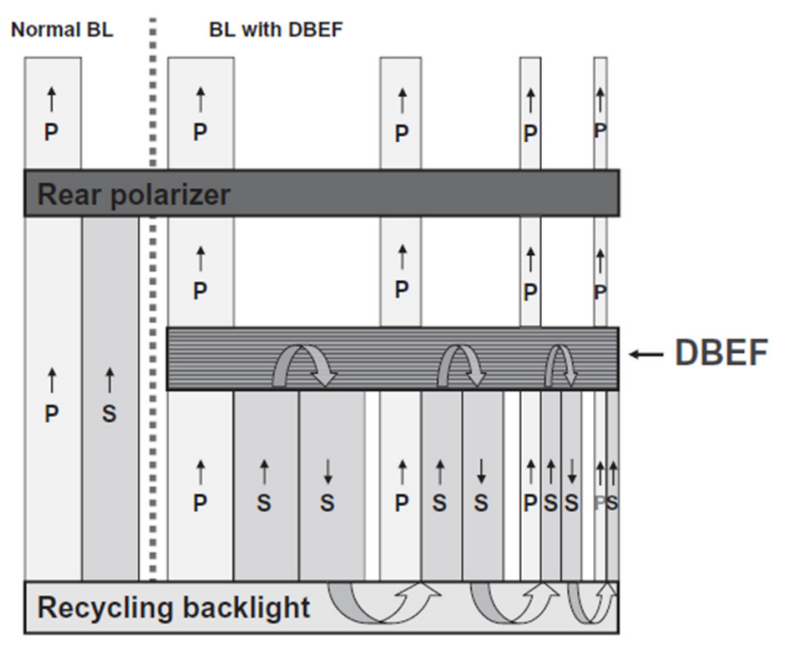

Source: Hanzawa 2009

Figure 4-11. Utilization of Light With and Without a DBEF

\section{c. Multi-function Films}

Optical films have been combined in many ways to reduce materials costs as well as to increase efficiency. The following combinations are either currently being used or are options that may soon be adopted:

\section{- Prism (BEF) + Reflective Polarizer (DBEF)}

$3 \mathrm{M}$ has recognized the cost barrier that manufacturers face in using DBEF and has provided a new proprietary film, DBEF-DTV, which combines BEF and DBEF, at a cost lower than the sum of the two standalone films.

\section{- Prism + Diffuser (Micro-lens Film)}

A micro-lens film is a new structure that incorporates diffusion and prism functions into one film. A microlens array scatters and defocuses light in a manner similar to the way diffusers function while also making the light converge as in a conventional lens. Recently, manufacturers have been adopting micro-lens films for some of their products as an alternative to 3M's DBEF or BEF. Figure 4-12 illustrates the mechanism of micro-lens films.

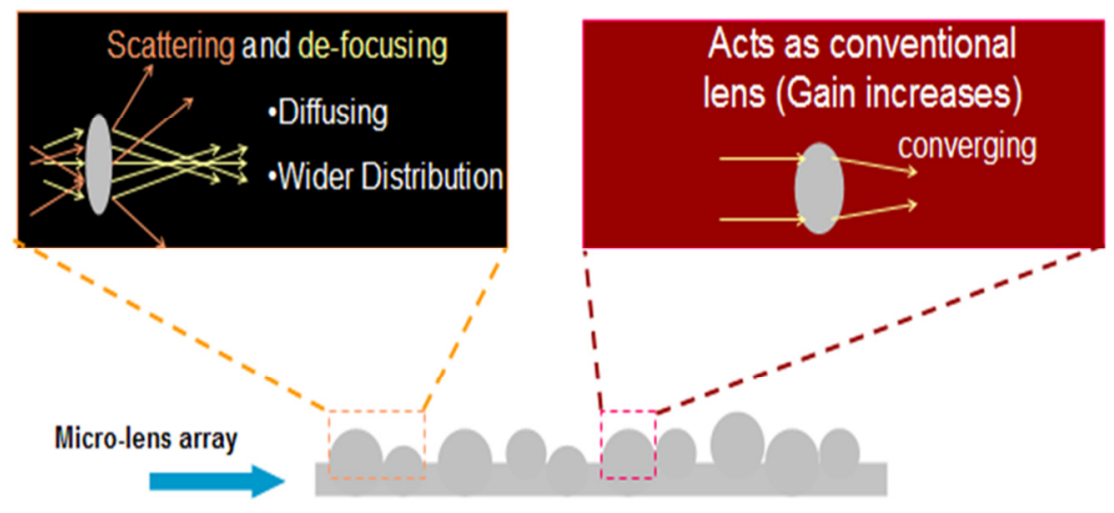

Source: DisplaySearch 2011c

Figure 4-12. Mechanism of Micro-lens Films 


\section{- Reflective Polarizer (DBEF) + Diffuser}

Recently, 3M has introduced a new series of DBEF, which combines DBEF and two diffuser layers, as an additional cost-effective solution for manufacturers. According to 3M, the new DBEF series exhibits the same brightness gain and transmittance as the conventional series (3M 2010b).

\section{- Light Guide Panel (LGP) + Prism}

According to an expert, energy efficiency can be improved by $10 \%$ by a lenticular-patterned or prismpatterned LGP. However, the materials cost for the new LGPs is expected to be $30 \%$ higher than the materials cost for conventional LGPs. Also, since the new structure still needs to overcome technical issues related to low uniformity of light distribution, it is not expected to be realized until 2012.

Among the various films discussed above, prism, lenticular, and micro-lens films are widely used in recent products. Table 4-7 compares prism, lenticular, and micro-lens film functions and costs.

\begin{tabular}{|c|c|c|c|}
\hline & Prism & Lenticular & Micro-lens \\
\hline Side view & & meresting & 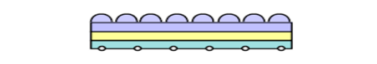 \\
\hline \multicolumn{4}{|l|}{ Top view } \\
\hline Optical Function & Light concentration & $\begin{array}{c}\text { Light concentration }+ \\
\text { diffusion }\end{array}$ & $\begin{array}{c}\text { Light concentration }+ \\
\text { diffusion }\end{array}$ \\
\hline Brightness Gain Factor & $\sim 1.6 \mathrm{x}$ & $\sim 1.5 \mathrm{x}$ & $\sim 1.3 \mathrm{x}$ \\
\hline Ease of Manufacture & Low & Medium & High \\
\hline Material Cost & High & Medium & Low \\
\hline
\end{tabular}

Source: DisplaySearch 2011c

Table 4-7. Various Optical Films

\section{d. Cost of Efficiency Improvements from Improved Optical Films}

Although it is hard to estimate improvement potential and incremental cost for panel technologies, categorizing options by optical components makes it easier to identify relationships between efficiency improvements and corresponding incremental costs. Currently, some TV manufacturers have removed DBEFs from their products, e.g., 32-inch $(81.3-\mathrm{cm}) 60-\mathrm{Hz}$ or $120-\mathrm{Hz}$ TVs but are still using DBEFs for other products with lower panel transmittance. (See Table 4-8) According to an expert, manufacturers anticipate that they will not need to use DBEF in the near future as panel transmittance improves and other combinations of optical films are developed. If manufacturers can achieve high efficiency without DBEF, there will be room to further improve efficiency by $20 \%$ to $30 \%$ (i.e., the efficiency improvement previously due to DBEF). By employing DBEF or using other approaches that entail additional costs, manufacturers can achieve higher efficiency than the level currently being targeted. If the efficiency of optical films improves, the initial luminance level currently required from backlight sources would not have to be as high. Consequently, manufacturers would be able to reduce the number of lamps and correspondingly reduce energy consumption. Other than adding DBEF to the film stack, it is hard to choose an optimal combination from other optical films because 1) film stacks in LCD BLUs vary widely among manufacturers, screen sizes, and models and 2) data are not available on the effect of each film stack on overall efficiency. Table 4-8 shows different types of film stacks for LED-edge backlight and CCFL backlight TVs, and Table 4-9 shows the incremental cost of adding DBEF to backlight TVs. 


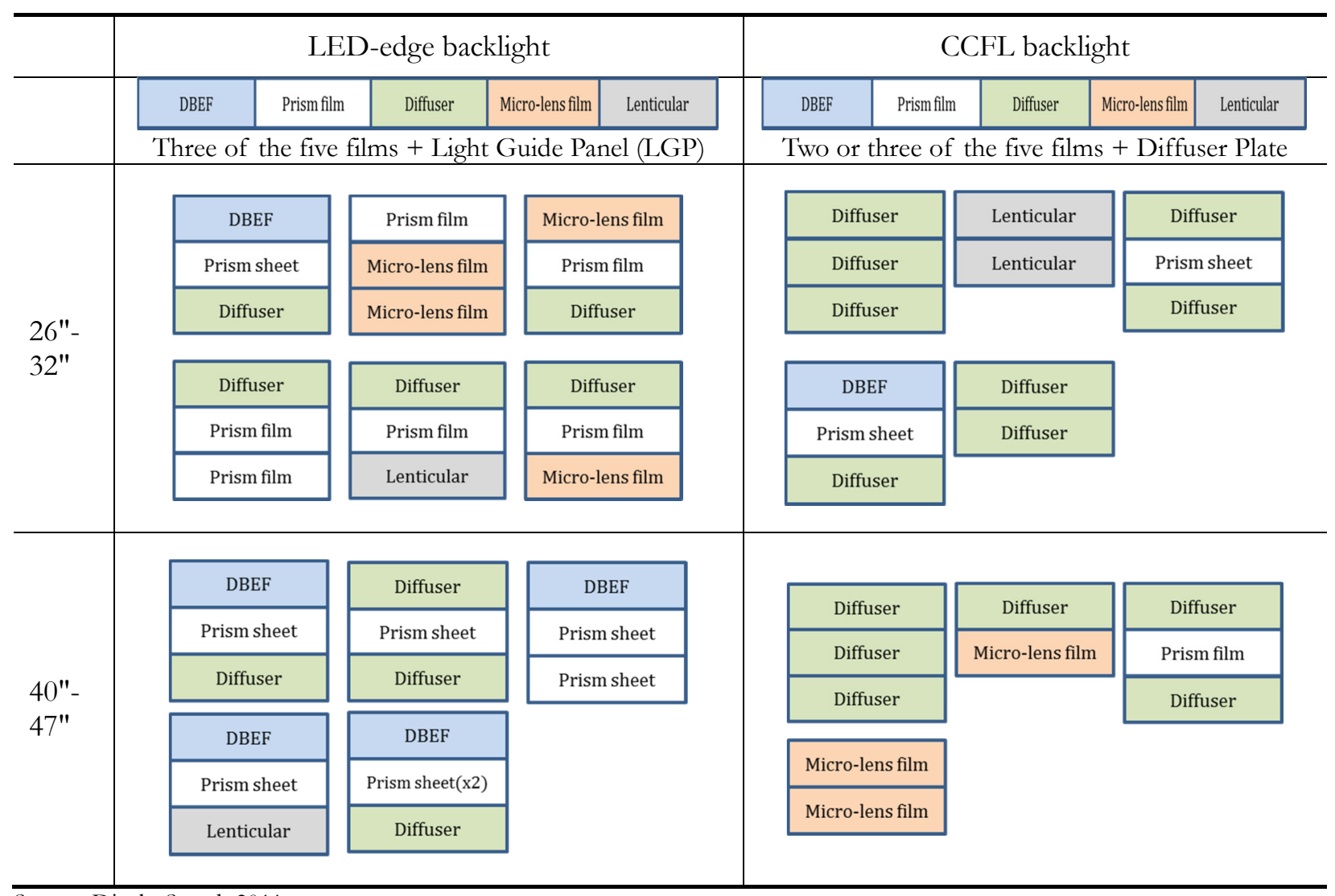

Source: DisplaySearch 2011c

Table 4-8. Various Film Stacks in BLUs ${ }^{48}$

\begin{tabular}{c|c|c}
\hline Screen Size & 2010 & $\begin{array}{c}\text { Cost-effective Option (2012) } \\
\text { (For backlight without DBEF) }\end{array}$ \\
\hline 32-inch & $\$ 7-\$ 8$ & $+\$ 4.9-5.6^{49}$ \\
\hline 40- to 42-inch & $\$ 12-\$ 13$ & $+\$ 8.4-\$ 9.1$ \\
\hline
\end{tabular}

Source: manufacturer, DisplaySearch 2010d

\section{Table 4-9. Cost of DBEF as a Cost-effective Option}

Additionally, a new structure that combines prism sheet and LGP is expected to increase efficiency by $10 \%$ compared to a conventional LGP. However, according to an expert, the new structure still needs to overcome technical issues related to low uniformity of light distribution and low viewing angle before it can be commercialized. The materials cost of a new LGP will be 30\% higher than the cost of a conventional LGP. Incremental costs for DBEF are expected as shown in Table 4-9, which is based on manufacturer information and the forecast that the materials cost of BLUs will decrease by 30\% from 2010 to 2012 (DisplaySearch 2010d).

48 According to DisplaySearch report (2011c), these examples are based on BLUs from Korean and Taiwanese manufacturers.

49 Cost of $\operatorname{DBEF}(2010) \times 0.7$ 


\section{E] Panel Efficiency Improvement: Transmittance Improvement}

\section{a. Liquid Crystal (LC) Cell Structures}

Most technologies for LCD panel efficiency improvement are proprietary, and they depend on LC cell structure, such as twisted nematic (TN), vertical alignment (VA) and in-plane Switching (IPS). Manufacturers have invested in R\&D to improve their own cell structures because LCD needed to overcome initial technical problems in viewing angle, transmittance, and response time to be optimized for application in TVs. In addition, manufacturers have an intrinsic motivation to improve panel efficiency because doing so will allow them to reduce the use of high-efficiency and expensive optical films and backlight lamps, which will reduce manufacturing costs. The subsections below describe the most common cell structures (3M 2010a, Baker 2011).

\section{- TN (Twisted Nematic): by Most Manufacturers}

The TN display is the most common type of LCD but was, until recently, available only for small screen sizes (less than 20 inches) because of significant image degradation at oblique angles. Recently, however, it has been improved with the help of supplementary films, expanding its availability for slightly larger screen sizes (20 to 26 inches). TN mode allows the LCD panel to have high transmittance and good yield; ${ }^{50}$ therefore, it offers the greatest benefits in energy efficiency and manufacturing cost of all LC cell structures. However, according to experts and manufacturers, applying the TN cell structure to medium-and-large screen sizes (greater than 30 inches) does not appear feasible because of its color tone degradation and contrast variation depending on viewing angle.

\section{IPS (In-Plane Switching): by Hitachi, Panasonic, Toshiba, and LG Display}

The first IPS structure was developed by Hitachi in 1996 to improve the viewing angle of an LCD (Baker 2011). Since then, there have been significant improvements in transmittance and contrast ratio. At an international exhibition in 2007, IPS-alpha Technology ${ }^{51}$ demonstrated that the light transmittance of IPSPro technology has improved by $80 \%$ over 10 years compared to the first version of IPS. LG Display also has significantly improved its panel transmittance during the past several years. According to experts and manufacturers, transmittance of early LCD panels is said to have been $2 \%$ to $3 \%$, while panel transmittance of existing IPS technologies is about $5 \%$ to $6.5 \%$. This is expected to improve to $7 \%$ to $8 \%$ by 2012 . Figure 4-13 shows the development of IPS.

\begin{tabular}{|c|c|c|c|c|c|}
\hline & IPS(1996) & S-IPS(1998) & AS-IPS(2002) & IPS-Pro(2005) & IPS-Pro(2007) \\
\hline Structure & & & & & \\
\hline Transmittance & $\begin{array}{r}100 \% \\
(2.5-2.8 \%) \\
\end{array}$ & $\begin{array}{r}100 \% \\
(2.5-2.8 \%) \\
\end{array}$ & $\begin{array}{r}130 \% \\
(3.3-3.6 \%) \\
\end{array}$ & $\begin{array}{r}160 \% \\
(4-4.5 \%) \\
\end{array}$ & $\begin{array}{r}180 \% \\
(4.5-5 \%) \\
\end{array}$ \\
\hline Contrast Ratio & $100 \%$ & $140 \%$ & $250 \%$ & $300 \%$ & $450 \%$ \\
\hline
\end{tabular}

*S-IPS: Super IPS, AS-IPS: Advanced Super IPS. Parentheses represent actual transmittance estimated by the author.

Source: Baker 2011, manufacturers

Figure 4-13. Development of IPS Structure

50 Yield is a measure of productivity, amount of output from a given amount of input.

51 IPS Alpha Technology was established by Hitachi, Matsushita (Panasonic) and Toshiba and became a subsidiary of Panasonic in 2010. 


\section{- Vertical Alignment (VA): Samsung, Sony, ${ }^{52}$ Sharp, AU Optronics, Chi Mei Optoelectronics}

VA technologies were developed by Fujitsu in 1996 to improve the viewing angle of an LCD. The most common type of VA panel, developed in 1998, is multi-domain vertical alignment, but each manufacturer has developed its own cell structure and named it differently. ${ }^{53}$ Current VA panel transmittance is reportedly about $5 \%$ to $6 \%$; this is expected to improve to $6 \%$ to $7 \%$ by 2012 .

IPS and VA technologies have been developed for TVs in medium and large screen sizes. Manufacturers are overcoming intrinsic problems in TN displays, such as image degradation at oblique viewing angle, by using supplementary films. This has resulted in TN displays being used for TV screens in the 20 -inch band.

\section{b. Panel Transmittance}

As mentioned above, the average transmittance of existing LCD panels is about $5 \%$ to $6.5 \%{ }^{54}$ and expected to increase to $6 \%$ to $8 \%$ by 2012 . Although panel transmittance varies with LC cell structure, display specification, and manufacturer, Table 4-10 presents averages forecasted by manufacturers. If panel transmittance improves, further brightness gain or efficiency improvement would be possible using the same film stack as before-improvement. Alternately, manufacturers could reduce the number of lamps rather than using high-efficiency optical films, which would reduce power consumption and costs. In addition, a highefficiency LCD structure can reduce the power needed to drive LCD panels. Assuming that final targeted luminance of an LCD TV panel would be $400 \mathrm{~cd} / \mathrm{m}^{2}$, then, as panel efficiency improves from $5 \%$ to $8 \%$, backlight power consumption can, in theory, decrease 36\% because of a reduced number of lamps. (See Table 4-11) Although the correlation between panel efficiency improvement and backlight lamp reduction is not straightforward in the field, it is clear that panel transmittance can positively and directly affect power consumption.

\begin{tabular}{l|c|c|c}
\hline & $2009-2010$ & $2011-2012$ & $2013-2015$ \\
\hline $\begin{array}{l}\text { Panel } \\
\text { Transmittance }\end{array}$ & $5-6.5 \%$ & $6-8 \%$ & $6-10 \%$ \\
\hline
\end{tabular}

Source: experts and manufacturers

Table 4-10. Forecast of Average LCD Panel Transmittance for TVs

\begin{tabular}{l|l|l|l|l|l}
\hline Target luminance of LCD panel & $\mathrm{cd} / \mathrm{m}^{2}$ & 400 & \multicolumn{5}{l}{} \\
\hline Panel transmittance & $\%$ & 5 & 6 & 7 & 8 \\
\hline Required luminance from backlight & $\mathrm{cd} / \mathrm{m}^{2}$ & 8,000 & 6,667 & 5,714 & 5,000 \\
\hline Number of necessary lamps & & $100 \%$ & $84 \%$ & $72 \%$ & $64 \%$ \\
\hline
\end{tabular}

Table 4-11. Effect of Panel Transmittance on LED Backlight Power Consumption

\section{c. Technology Options for Panel Transmittance Improvement \\ - High aperture ratio: improved cell structure, low-voltage driven materials}

In general, panel transmittance can be improved by enlarging cell aperture. For example, while black matrix is used to prevent light leakage from the gaps between pixels, which improves resolution, it also blocks light

\footnotetext{
52 Sony has obtained LCD panels from Samsung LCD, and will obtain them from Sharp in the future.

53 Samsung (PVA: Patterned Vertical Alignment), Sharp (ASV: Advanced Super View), AU Optronics (P-MVA: Premium multidomain vertical alignment, AMVA: Advanced MVA), Chi Mei Optoelectronics (S-MVA: Super MVA)

54 LCD panel transmittance varies among manufacturers and models. According to some manufacturers, 100/120-Hz driven LCD panels have $5 \%$ to $6 \%$ transmittance on average, and $200 / 240-\mathrm{Hz}$ driven LCD panels, including 3D-enabled LCDs, have $4 \%$ to $5 \%$ transmittance on average. In general, more efficient optical films or adjustment of the backlight structure compensate for lower transmittance.
} 
transmittance. Data bus lines are necessary for electric current flow, but the width of data lines reduces the actual cell aperture ratio. Even though manufacturers can achieve higher transmittance by adjusting these parts, new cell structures should not cause panel performance and productivity to diminish. Because cell structure is the most technically complex element in TFT-LCD manufacture, changing cell structures requires further R\&D investment.

One recent technology trend in panel efficiency improvement is development of low-voltage driven panels. For this technology, LC materials that can be driven by low voltage are needed. These LC materials would allow manufacturers to use narrower low-resistance data lines, i.e., high cell aperture ratio, than can currently be used. According to experts, aluminum-molybdenum (Al-Mo) is now being used for data lines and can be replaced with copper-titanium (Cu-Ti). In addition, panels should be developed with low-voltage drive schemes. The resulting low-resistance data lines will improve panel transmittance, and panels can also employ low-voltage-driven electric parts in the circuitry, which will reduce circuitry power consumption. Because development of low-voltage-driven panels is closely associated with LC materials, data line materials, cell structure, and the drive scheme, it is difficult to precisely and accurately estimate R\&D investment or incremental cost.

For small screen sizes (less than 26 inches $/ 66 \mathrm{~cm}$ ), manufacturers are likely to employ a combination of LED backlight and TN cell structure. According to one manufacturer, a 21-inch (53.3-cm) LED backlight TV panel with additional optical film (DBEF) is twice as efficient as a CCFL backlit TV panel of the same size.

\section{d. Cost of Panel Transmittance Improvement}

As mentioned above, it is difficult to estimate the incremental cost and R\&D investment for the development of new LC cell structures because 1) technology options are complex and interwoven, 2) data are not readily available, and 3) each manufacturer has its own proprietary technology. A further benefit from low-voltage panel development is reduced cost for drive circuitry because low-voltage-driven components are less expensive than high-voltage-driven ones. Manufacturers have historically focused on panel transmittance improvements and may continue to do so because of the intrinsic motivation to reduce manufacturing costs by reducing the number of backlight lamps or optical films used.

\section{F] Power Management in On-Mode: Dynamic Dimming}

\section{a. Dimming Methods}

Backlight dimming methods have been developed to reduce power consumption and improve image quality in terms of contrast ratio. An advantage of LED backlights compared to CCFL backlights is that LED backlight lamps can be more finely controlled by a dimming algorithm. To employ dimming, LED drive circuitry needs to keep the current across LED channels uniform while modulating the backlight brightness data using the dimming algorithm (Kwon et al. 2010).

The simplest dimming option is to dim the whole backlight by a universal factor in each frame, which is called zero-dimensional (0D), complete, or global dimming. This option can be applied to all types of backlights. Another option is to dim part of the backlight area depending on input image, which is called partial or local dimming. Local dimming methods can individually control each lamp or LED block; for this method, LEDdirect backlights have an advantage over LED-edge and CCFL backlights. However, LED-edge backlights are expected to dominate the LCD TV market; LED-edge backlighting can effectively reduce power consumption by dimming each block of LEDs. Figure 4-14 shows global and local dimming systems. 


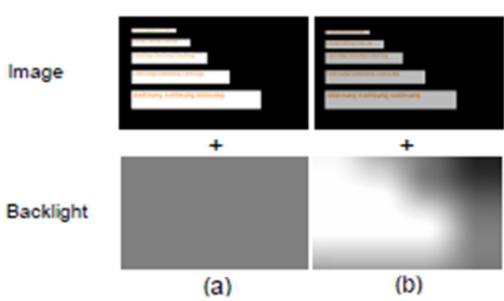

Figure 1. Global dimming and local dimming (a) Global dimming (b) Local dimming

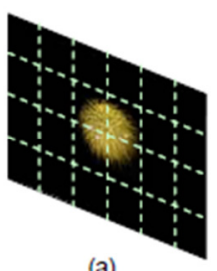

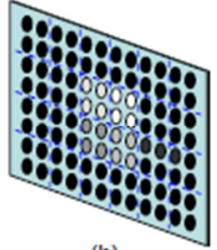

Figure 2. Local dimming system

(a) Image blocks (b) LED blocks

Source: Chen 2006

\section{Figure 4-14. Global Dimming and Local Dimming}

Broadly, manufacturers and researchers refer to three types of dimming methods using various terms for each:

1) OD dimming: zero-dimensional dimming, complete dimming, global dimming

2) 1D dimming: 1-dimensional dimming, line dimming, partial dimming

3) 2D dimming: 2-dimensional dimming, local (point, block) dimming

Table 4-12 shows the effect of dynamic dimming on power consumption for LED backlit TVs.

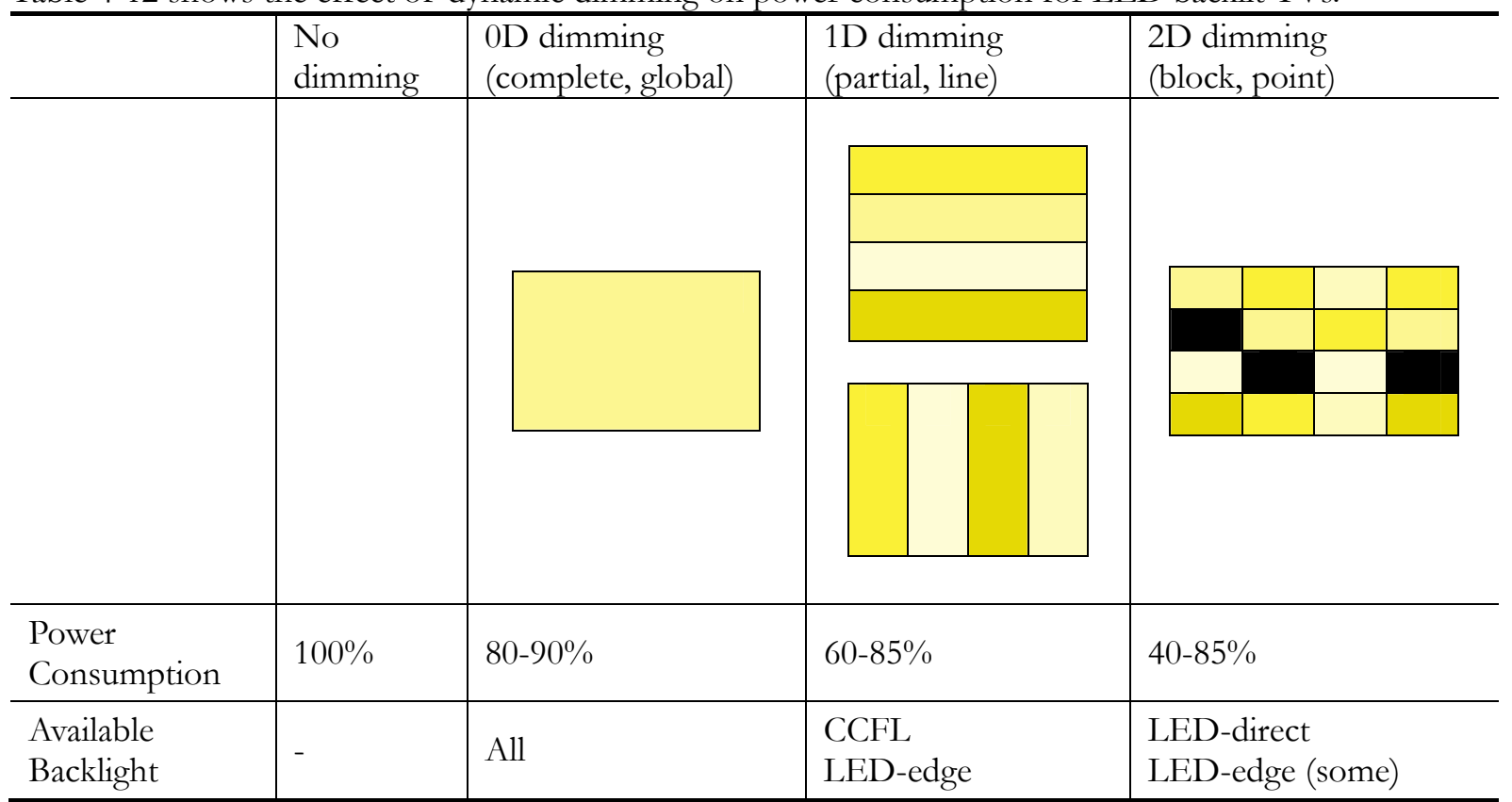

Table 4-12. Average Effect of Dynamic Dimming on Power Reduction in LED Backlit TV

Although local dimming has the most significant effect on power consumption, LED-edge backlit TVs can use only $1 \mathrm{D}$ or block dimming techniques. Assuming the same dimming method, the effect of dimming on power consumption is determined mainly by backlight segmentation and input images (both average picture level and image forms). The effect of dimming on power consumption is very significant for dark images with low brightness levels but not that significant for bright images. According to one manufacturer, a global dimming method can reduce power consumption by $19 \%$ for a sample image, and $1 \mathrm{D}$ dimming can reduce power consumption by $41 \%$ for the same image. To quantify the effect of dimming, it is necessary to measure power consumption using various dimming methods with the IEC 62087 standard test video clip. Reliable data of this type are not available for this analysis. We assume an average effect of dimming methods based on interviews and materials from manufacturers. 


\section{b. Improvements in Dimming Technology}

Because the effect of dimming methods can vary with input images, dimming algorithm, and backlight structure, it is not easy to quantify precisely the average effect of dimming methods. To obtain reliable data, measurements must be done using the IEC 62087 standard video clip. Considering that LED-edge backlights are currently expected to dominate the market over direct-type backlights, $1 \mathrm{D}$ dimming or partial dimming methods would have more impact than local dimming methods that are only available to LED-direct type backlights. Recently, some manufacturers have been exploring the 1.5D dimming method. This method combines 1D dimming with a scanning backlight, which is also used to reduce the motion blur ${ }^{55}$ problem in LCD TVs. Use of $1.5 \mathrm{D}$ dimming adds a dark stripe in the direction the display is rendered; consequently, it can affect power consumption. The scope of dimming methods for edge backlights might be limited as the backlight structure becomes simpler, but reducing the number of backlight lamps can also reduce power consumption, and the tradeoff between these two issues and the optimal design for high efficiency is unclear, complex, and beyond the scope of this analysis. We illustrate this tradeoff in Table 4-13.

\begin{tabular}{|c|c|c|c|c|}
\hline \multirow[b]{2}{*}{ Structure } & 4 sides & \multicolumn{2}{|c|}{2 sides } & 1 side \\
\hline & & & & i \\
\hline Dimming & Local dimming & \multicolumn{2}{|c|}{ Line dimming, Block dimming } & Line dimming \\
\hline $\begin{array}{l}\text { Effect on power } \\
\text { reduction }\end{array}$ & Better & \multicolumn{2}{|c|}{ Good } & Fair \\
\hline \# of LEDs & $100 \%$ & \multicolumn{2}{|c|}{$40-60 \%$} & $25-30 \%$ \\
\hline $\begin{array}{l}\text { Product Segment } \\
\text { (as-is) }\end{array}$ & Flagship (>46") & $\begin{array}{l}\text { High-end } \\
\left(52 "-55^{\prime \prime}\right)\end{array}$ & $\begin{array}{l}\text { Main-stream } \\
(40 "-47 ")\end{array}$ & $\begin{array}{c}\text { Entry (32"-37") } \\
\text { Main-stream (40"-47") }\end{array}$ \\
\hline
\end{tabular}

Source: manufacturers, DisplaySearch 2011c

Table 4-13. Structures of LED-edge Backlight and Dimming Methods

\section{c. Cost of Dimming Technology}

Manufacturers employ dimming technology mostly for mid-range or high-end products because of the incremental cost. Low-end products have only $0 \mathrm{D}$ dimming or no dimming option. Although dimming methods can improve picture quality in terms of contrast-ratio, some manufacturers express uncertainty about whether the dimming effect is fully perceived by consumers. In addition, a certain type of block dimming causes color balance across the screen to deteriorate for some types of scenes.

To employ dimming methods, manufacturers need to use drive integrated circuits (ICs) that control the dimming algorithm, at an additional cost of $\$ 0.8-\$ 1.5$ per drive IC. The necessary number of drive ICs varies from one to six according to screen size. Although additional analysis is needed to determine the average effect of dimming methods, dimming technology is a good candidate for a market transformation program. Table 4-14 shows the incremental costs of dimming options.

\footnotetext{
${ }^{55}$ Motion blur is the streaking of a rapidly moving object across the LCD display. This phenomenon results mainly from the intrinsically slow response time of liquid crystals.
} 


\begin{tabular}{c|c|c}
\hline Screen Size & $\begin{array}{l}\text { Usage of } \\
\text { Drive ICs }\end{array}$ & Incremental Cost* \\
\hline $20 "-29 "$ & 1 & $\$ 0.8-\$ 1.5$ \\
\hline $30 "-39 "$ & 4 & $\$ 3.2-\$ 6.0$ \\
\hline $40 "-49 "$ & 4 & $\$ 3.2-\$ 6.0$ \\
\hline $50 "+$ & 6 & $\$ 4.8-\$ 9.0$ \\
\hline
\end{tabular}

*Costs based on the year 2010

\section{Table 4-14. Incremental Cost of Dimming Options}

\subsubsection{Summary of Efficiency Improvement Options in LCD TVs}

\section{A] Forecast for Efficiency Improvement (Market Potential)}

Based on the above discussion, we conclude that LCD TV efficiency is expected to improve in the following key technical areas: LED efficacy, panel transmittance, and optical films. LED efficacy and panel transmittance improvements will play a significant role in reducing power consumption and manufacturing costs. Table 4-15 shows the efficiency forecast for 32-inch LCD TVs.

\begin{tabular}{l|l|l|l|l}
\hline & & \multicolumn{1}{|c|}{2010} & \multicolumn{1}{c}{2011} & \multicolumn{1}{c}{2012} \\
\hline BLU & LED-Edge & $\begin{array}{l}\text { 1side(Horizontal: H) } \\
2 \text { sides (Vertical: })\end{array}$ & $\begin{array}{l}\text { 1side(H) } \\
2 \text { sides }(\mathrm{V})\end{array}$ & $\begin{array}{l}\text { 1side(H) } \\
2 \text { sides }(\mathrm{V})\end{array}$ \\
\hline \multicolumn{1}{c|}{ LED Spec. } \\
$\begin{array}{l}\text { Panel } \\
\text { Transmittance }\end{array}$
\end{tabular}

* DS (Diffuser Film), PR (Prism Film), MF (Micro-lens Film), LE (Lenticular Film)

\section{Table 4-15. Example of Efficiency Improvement Forecast for 32-inch LCD TVs}

Although the efficiency of panel transmittance and optical films is expected to improve, it is likely that manufacturers will employ new combinations of films, removing DBEF from the conventional stack to reduce materials costs. In addition, the effect of dimming methods on power consumption is significant, but it is likely that dimming technology will be adopted only in mid-range/high-end products because of its incremental cost. This forecast applies to medium and large screen sizes, i.e., larger than 30 inches $(76.2 \mathrm{~cm})$.

\section{B] Opportunities for Efficiency Improvement through Market Transformation (Economic Potential)}

The relationship between efficiency improvement and corresponding incremental cost is clear for only a few technologies because most efficiency improvement options are closely associated with panel technologies and are complex, interconnected, and involve proprietary technology.

Most panel-related technologies require R\&D investment because, in FPDs such as LCD, PDP, OLED, etc., panel design is the most complicated element and therefore the most expensive to modify. However, because advanced panel design can provide huge benefits to manufacturers in the form of manufacturing cost reductions, the industry has put much effort into R\&D to improve these technologies. 
Nonetheless, improved optical films and dimming options are good candidates for a market transformation program because these are mature technologies that are already commercially available, with well-known and predictable costs.

As discussed above, LED-LCD TV panel efficiency is expected to improve by $25 \%$ to $35 \%$ in the next 2 to 3 years even in the absence of a market transformation program. The results of this efficiency improvement could be reduced power consumption (from employing fewer LEDs) or reduced costs (from removing expensive optical films). The change will be driven by new efficiency standards and manufacturers' intrinsic motivation to reduce cost and improve performance.

Assuming that TV manufacturers achieve the same efficiency without DBEF in many of their entry-level products within the next few years, then adding DBEF to those units could be an option to further improve efficiency. It is also possible that manufacturers will identify other options that have the same end result.

Dimming technology is another option to further improve efficiency. Although the effect of dimming methods on power consumption reduction needs to be measured with a standard video clip and is not precisely quantifiable in this report, it is expected to be significant even for LED-edge backlights. Because manufacturers are expected to employ the option only in mid-range or high-end products, this option could be encouraged to improve the efficiency of entry-level models. Figure 4-15 summarizes the cost-effective options for entry-level 32-inch LED-LCD TVs.

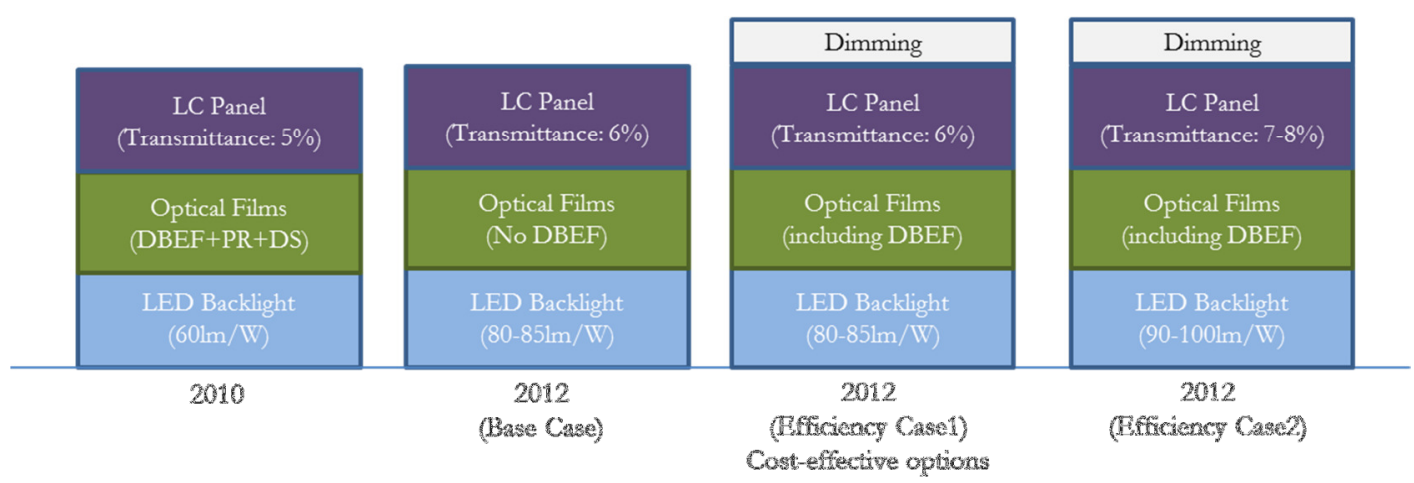

* DS (Diffuser Film), PR (Prism Film), MF (Micro-lens Film)

Figure 4-15. Cost-effective Options for 32-inch Entry-level LED-LCD TVs

Although Efficiency case 2 can be assumed, this report considers only Efficiency case 1 as incremental costs for improvement in panel transmittance and high efficacy LEDs are not available. Based on this scenario, average on-mode power of 32-inch LED-edge backlit LCD TVs is estimated as follows. The average onmode power of 32-inch ENERGY STAR LED-edge backlit TVs sorted out of ENERGY STAR 2010 is 54 $\mathrm{W}^{56}$ It is expected that the on-mode power of 32-inch LED-edge LCD TVs will be reduced to $44 \mathrm{~W}$ by improvements in LED efficacy and panel transmittance by 2012. If the TVs do not have a backlight dimming option and DBEF, both options can be added, which will entail additional material costs but on-mode power will be further reduced to $39 \mathrm{~W}$. Figure 4-16 shows on-mode power estimates for 32-inch LED-edge backlit TVs.

\footnotetext{
56 Average of the ENERGY STAR-qualified TVs whose ABC function enabled when they are shipped. The average on-mode power of 32-inch LED backlit TVs in ENERGY STAR TVs listed on March, 2011 is $53 \mathrm{~W}$.
} 


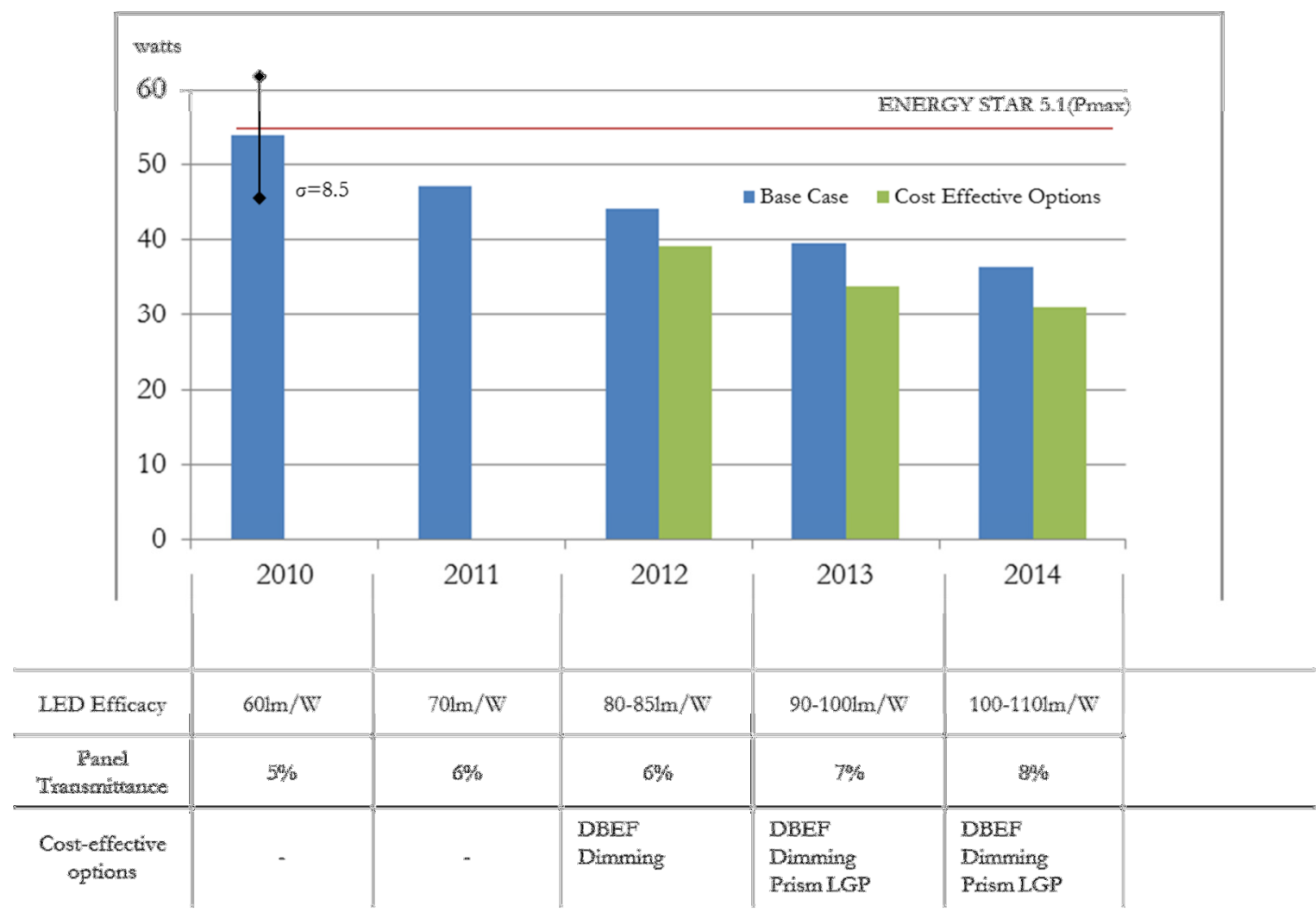

Figure 4-16. Estimates of On-mode Power for 32-inch LED Backlit TVs

The average cost of DBEF for a 32-inch LCD TV in 2010 was approximately $\$ 7$ to $\$ 8$. Assuming that the DBEF option can reduce the on-mode power of a 32-inch LED-LCD TV by $5 \mathrm{~W}$, the cost of conserved energy would be $\$ 0.08$ per $\mathrm{kWh}$ assuming a discount rate of $6 \%$ and an effective useful life of 10 years. Although the average effect of dimming options in LED-edge backlights is difficult to calculate precisely, adding a dimming option (0D or 1D) would further reduce BLU power consumption by $15-50 \%$. An industry expert indicates that it would cost an additional $\$ 3.2$ to $\$ 6.0$ to employ dimming-enabled drive ICs for 30 - to 47-inch LCD TVs. Details calculations of cost of conserved energy for these cost-effective options are discussed in Appendix D. 


\subsection{Plasma Display Panel (PDP) TV}

In this subsection we discuss key factors affecting efficiency improvements in PDP TVs, technology options to improve efficiency in PDP TVs, and possible manufacturing cost reductions if these technology options are realized. We also summarize opportunities for efficiency improvement in PDP TVs.

\subsubsection{Key Factors Related to Efficiency Improvement in PDP TVs}

\section{A] Features of PDP Power Consumption}

PDPs are self-emissive displays that convert ultraviolet (UV) radiation, generated when a gas discharge excites phosphors, to visible light in each cell. Because of its self-emissive nature, improvement in a PDP's luminous efficacy is the key to saving energy in PDP TVs. In addition, because the transmittance of PDP filters is currently $40 \%$ to $48 \%$, improvement in filter transmittance will lower the target luminance level that bare panels need to achieve, reducing power consumption. Because manufacturers report that the efficiency of the PSU for PDPs is now between 85\% and 95\%, the PDP panel and filter are the most important candidates for efficiency improvement. Although PDP target luminance varies across models and manufacturers, one manufacturer estimates that the average luminance of current 42-inch $(106.7-\mathrm{cm})$ HD PDP panels without filter is about 160 to $180 \mathrm{~cd} / \mathrm{m}^{2}$ (at $100 \% \mathrm{APL}^{57}$ ); these panels consume $180 \mathrm{~W}$ each at that luminance level. For the whole TV set, total power consumption is estimated to be more than $200 \mathrm{~W}$. Table 4-16 shows the luminance of key components and the complete unit for a 42-inch HD PDP TV.

\begin{tabular}{l|l|r}
\hline \multirow{2}{*}{ Set } & Key Components & $\begin{array}{l}\text { Luminance in full white mode at } \\
100 \% \text { APL }\end{array}$ \\
\hline \multirow{2}{*}{ Module } & $\begin{array}{l}\text { Digital Signal Processing } \\
\text { Power Supply Unit }\end{array}$ & $54-82 \mathrm{~cd} / \mathrm{m}^{2}(30-46 \%)$ \\
\cline { 2 - 3 } & $\begin{array}{l}\text { Filter (anti-reflection, color adjustment })^{59} \\
\text { Priving Circuitry }\end{array}$ & $64-86 \mathrm{~cd} / \mathrm{m}^{2}(40-48 \%)$ \\
\hline
\end{tabular}

* The final luminance of the panel is regarded as the starting point, $100 \%$.

Therefore, this definition of efficiency does not capture the luminous efficacy of the panel itself.

Table 4-16. Luminance of a 42-inch (106.7-cm) HD PDP TV

In fact, "default-luminance as shipped" of 42-inch $(106.7-\mathrm{cm})$ ENERGY STAR-listed HD PDP TVs (ENERGY STAR 2010) is between $53 \mathrm{~cd} / \mathrm{m}^{2}$ and $96 \mathrm{~cd} / \mathrm{m}^{2}$, which supports the above estimate. Table 4-17 shows on-mode power consumption of those ENERGY STAR-qualified PDP TVs is between $90 \mathrm{~W}$ and 113 W. Although PDP TVs consume less power at low APL, and the IEC 62087 broadcast-content test video signal, which is used to measure on-mode power consumption, has a 34\% APL on average (Waide 2011), the on-mode power values in the ENERGY STAR list appear low, considering that PDPs' power consumption is typically saturated at more than $40 \%$ of APL (Figure 4-17).

\footnotetext{
57 Average brightness of TV programs is between $20 \%$ and $50 \%$ APL.

58 PSUs can be installed in panel manufacturing process or TV set production.

59 Conventional glass filters were installed by TV set makers, but recent film filters are installed during the panel manufacturing process.
} 


\begin{tabular}{l||l|l|l||l|l|l}
\hline Screen Size & Resolution & $\begin{array}{l}\text { Number of } \\
\text { Models }\end{array}$ & $\begin{array}{l}\text { On-mode } \\
\text { Power (W) }\end{array}$ & Resolution & $\begin{array}{l}\text { Number of } \\
\text { Models }\end{array}$ & $\begin{array}{l}\text { On-mode } \\
\text { Power (W) }\end{array}$ \\
\hline $\begin{array}{l}\text { 42inch } \\
(106.7 \mathrm{~cm})\end{array}$ & $\begin{array}{l}1024 \times 768 \\
1366 \times 768\end{array}$ & 5 & $\begin{array}{l}90-113 \\
108-112\end{array}$ & $1920 \times 1080$ & 4 & 99 \\
\hline
\end{tabular}

All TVs shipped with ABC enabled.

Table 4-17. 42-inch (106.7-cm) HD PDP TVs (ENERGY STAR 2010)

Figure 4-17 shows that ENERGY STAR-qualified PDP TVs appear to have achieved luminous efficacy greater than $3 \mathrm{~lm} / \mathrm{W}$. However, the luminous efficacy of a PDP panel that consumes $180 \mathrm{~W}$ at $180 \mathrm{~cd} / \mathrm{m}^{2}$ $\left(100 \%\right.$ APL) corresponds to about $2.6 \mathrm{~lm} / \mathrm{W}^{60}$ Possible explanations for this discrepancy are: 1) the luminance at default setting was low enough to consume less power at the test video signal. In fact, of the 65 TVs qualified for ENERGY STAR Version 4, 63 had ABC enabled when they were shipped. 2) Power consumption under 20\% APL (line A in Figure 4-17) has become very low, making the slope of line B steeper.

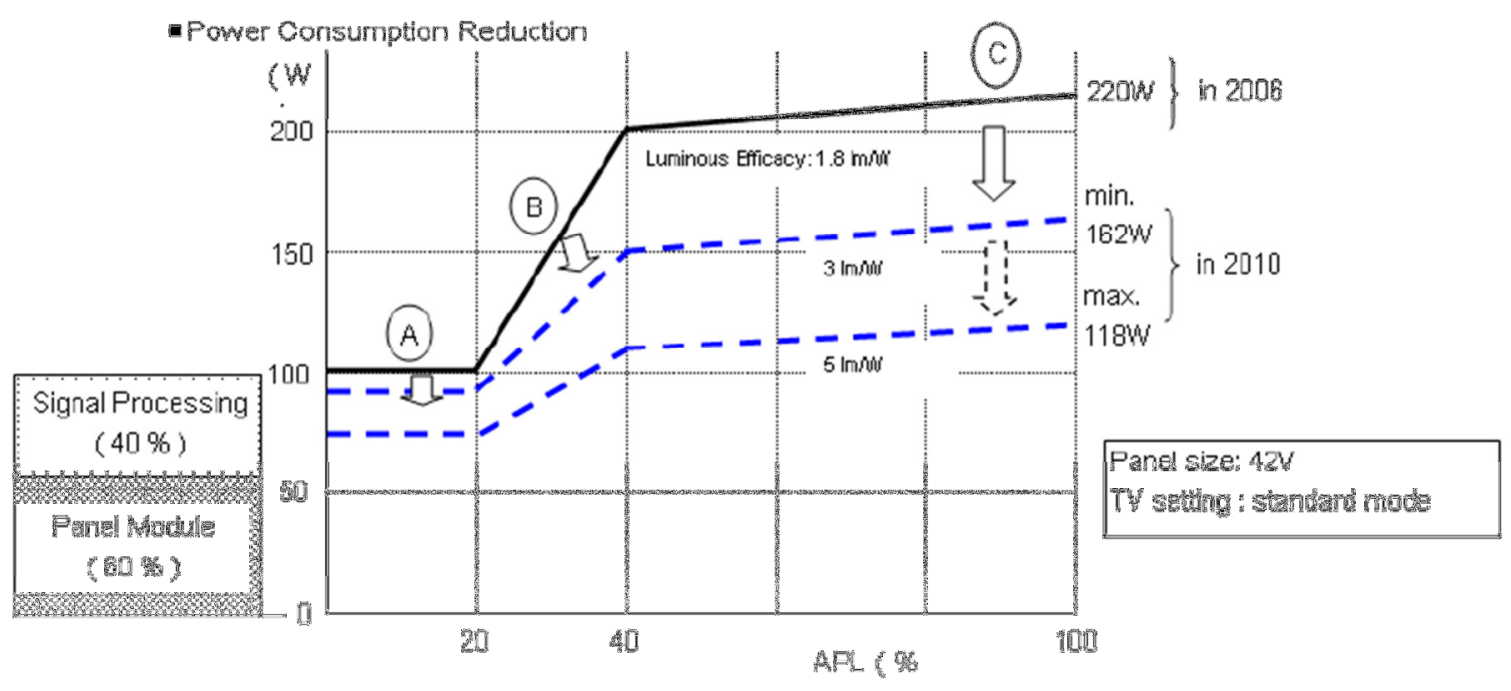

Source: Fraunhofer 2007d

\section{Figure 4-17. Power Reduction by Luminous Efficacy Improvement}

\section{B] Resolution and Power Consumption}

The luminous efficacy of full HD (1,080progressive [p] $)^{61}$ TVs is generally accepted to be lower than that of HD TVs (720p) because finer cell structure, more electrodes, more electric components are required for a high resolution panel. Panasonic TVs, which qualify for ENERGY STAR Version 4, show this relationship for on-mode power consumption although the data cannot be used to compare luminance level at the same on-mode power. Figure 4-18 shows that full HD TVs consume 10\% to $16 \%$ more power than HD TVs. This percentage increases by screen size, but there are no HD TVs (720p) with a screen size greater than 50 inches $(127 \mathrm{~cm})$ on the market.

${ }^{60}$ Luminous efficacy of a PDP has been calculated by the following formula.

$$
\frac{l m}{W}=\frac{\left(\text { average brightness, } \frac{c d}{m^{2}}\right) \times\left(\text { screen area, } m^{2}\right) \times(\pi)}{\text { net input power consumed for panel discharge }(W)}
$$

${ }^{61}$ Full HD TVs: $1920 \times 1080$ pixels, HD TVs: $1024 \times 768,1366 \times 768$ pixels 


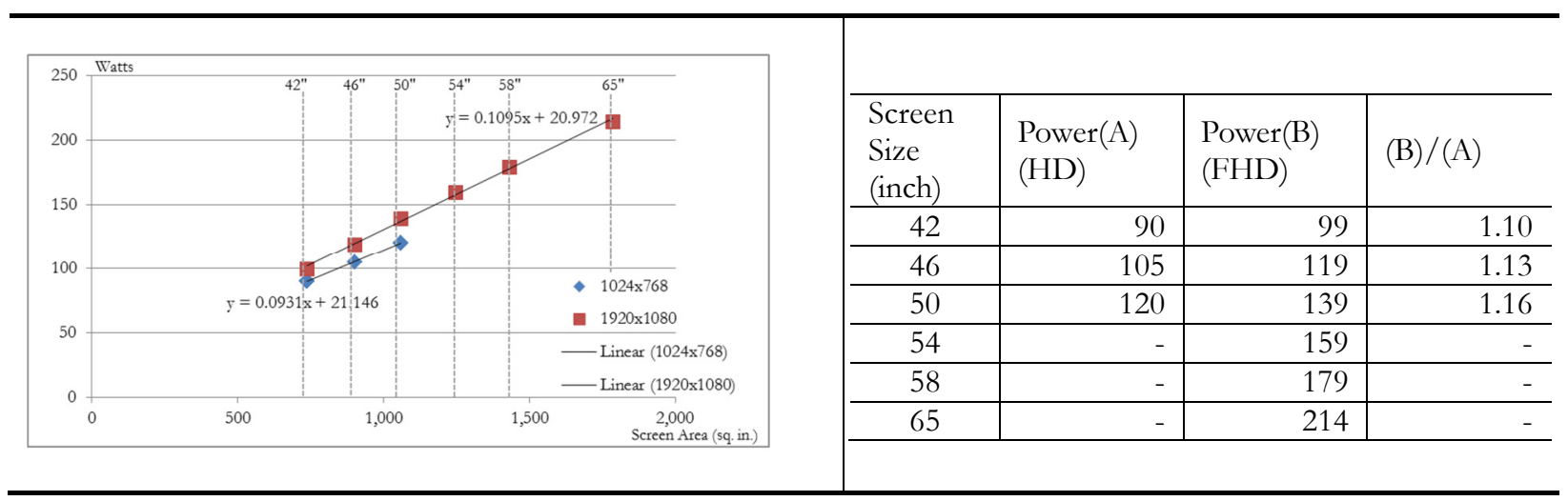

Source: ENERGY STAR 2010

Figure 4-18. On-Mode Power of Panasonic PDP TVs

Table 4-18 shows PDP screen size and cell pitch. To increase number of pixels for high resolutions, the cell size, or cell pitch, must become smaller in the same size. Since more electrodes and more barriers ribs are required, the total space that is allowed for light coming from cells should be smaller. As a result, high resolution PDPs consumer more power than low resolution PDPs to keep the same luminance level.

\begin{tabular}{|c|c|c|c|c|c|}
\hline \multirow{2}{*}{$\begin{array}{l}\text { Screen } \\
\text { Size }\end{array}$} & \multirow{2}{*}{$\begin{array}{l}\text { Display Area } \\
(\mathrm{mm} \times \mathrm{mm})\end{array}$} & HD & HD & Full HD & Ultra-Fine \\
\hline & & $\begin{array}{l}1024 \times 768 \\
(0.79 \mathrm{M} \text { pixels })\end{array}$ & $\begin{array}{l}1366 \times 768 \\
(1.05 \mathrm{M} \text { pixels })\end{array}$ & $\begin{array}{l}1920 \times 1080 \\
(2.07 \mathrm{M} \text { pixels })\end{array}$ & $\begin{array}{l}4096 \times 2160 \\
(8.85 \mathrm{M} \text { pixels })\end{array}$ \\
\hline $\begin{array}{l}\text { 42-inch } \\
(106.7-\mathrm{cm})\end{array}$ & $934 \times 532$ & 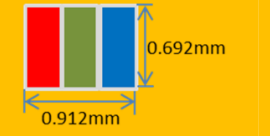 & 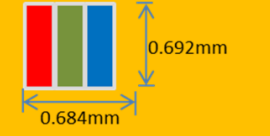 & $0.486 \mathrm{~mm}$ & - \\
\hline $\begin{array}{l}50 \text {-inch } \\
(127.0-\mathrm{cm})\end{array}$ & $1106 \times 622$ & $\underset{1.08 \mathrm{~mm}}{\mid} \underbrace{0.810 \mathrm{~mm}}$ & $\underset{0.810 \mathrm{~mm}}{\stackrel{\Downarrow}{\mid}} \mid$ & $\underset{0.576 \mathrm{~mm}}{\mid}>\mid$ & - \\
\hline $\begin{array}{l}\text { 63-inch } \\
(160.0-\mathrm{cm})\end{array}$ & $1394 \times 784$ & $\underset{1.36 \mathrm{~mm}}{\mid} \underline{v}^{1.02 \mathrm{~mm}}$ & 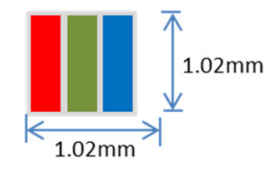 & 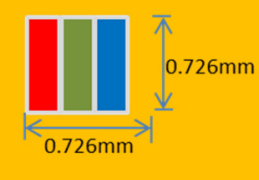 & 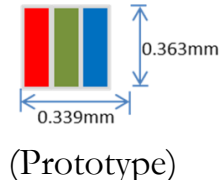 \\
\hline
\end{tabular}

Authors' calculation, M: million

: Currently commercially available

Table 4-18. PDP Screen Size and Cell Pitch (not to scale)

Although high-resolution panels consume more power than lower-resolution panels for the same screen size, the technologies for fine cell structure can positively affect efficiency improvement in lower-resolution panels. One of key technologies required for fine cell structure is manufacturing slimmer and physically stable barrier ribs. The technologies can allow PDPs to have higher cell aperture ratio in the same cell pitch, which results in higher efficiency. At Flat Panel Display (FPD) International 2008, Samsung SDI demonstrated a 63-inch $(160-\mathrm{cm})$ PDP TV featuring $4 \mathrm{k} \times 2 \mathrm{k}\left(4,096 \times 2,160\right.$ pixels $\left.^{62}\right)$, with a pixel size of $0.339 \mathrm{~mm} \times 0.363 \mathrm{~mm}$ (Otani 2008). Considering that the cell pitch of a commercialized 42 -inch $(81.3-\mathrm{cm})$ full HD TV is $0.49 \mathrm{~mm}$, other PDPs could improve luminous efficacy by increasing cell aperture ratio using similar technology.

In the short term, HD and full HD resolution will be the mainstream resolutions for TVs. In addition, PDP

62 One pixel consists of Red/Green/Blue cells. 
manufacturers are not currently producing HD TVs with larger than 50-inch screens. HD PDP TVs are now commercially available with screens from 42 inches to 50 inches, and larger-screen TVs are designed as flagship products for each TV brand. PDP manufacturers may not want to launch TVs with higher resolution than available in full HD because higher-resolution TVs consume more power, and PDP TVs are positioned in the large-screen area, which will be strictly regulated by ENERGY STAR Version $5{ }^{63}$ Recently, Panasonic started providing 50-inch HD PDP TVs with $1024 \times 768$ pixels even though the resolution corresponds to a 4:3 aspect ratio. Because the $1024 \times 768$ resolution has an efficiency advantage over HD which has more pixels, e.g., $1366 \times 768$ in the same screen size because higher cell aperture ratio, less electrodes, and less electric components are required, power consumption on 50-inch HD PDP TVs can be decreased. Table 4-19 shows the screen resolution and aspect ratio of commercially available HD PDP displays.

\begin{tabular}{c|c|c|c|c}
\hline \multirow{2}{*}{$\begin{array}{c}\text { Screen Size } \\
\text { (inch) }\end{array}$} & $\begin{array}{c}\text { Display Area } \\
(\mathrm{mm} * \mathrm{~mm})\end{array}$ & HD & HD & Full HD \\
\cline { 3 - 5 } & $934 \times 532(\sim 16: 9)$ & $\mathrm{O}$ & $\begin{array}{c}1366 \times 768 \\
(16: 9)\end{array}$ & $\begin{array}{c}1920 \times 1080 \\
(16: 9)\end{array}$ \\
\hline 42 & $1106 \times 622(16: 9)$ & New $(2010)$ & $\mathrm{O}$ & $\mathrm{O}$ \\
\hline 50 & $1394 \times 784(16: 9)$ & $\mathrm{X}$ & $\mathrm{O}$ & $\mathrm{O}$ \\
\hline 63 & $\mathrm{O}$ & $\mathrm{X}$ & $\mathrm{O}$ \\
\hline
\end{tabular}

O: Available on the market, X: Not available on the market

\section{Table 4-19. Commercially Available PDP TV Screen Resolution and Aspect Ratio}

Most PDP TV screens have a 16:9 aspect ratio, and the corresponding resolutions are 1366×768 pixels (HD) and $1920 \times 1080$ pixels (full HD). Although an HD resolution of $1024 \times 768$ pixels corresponds to a 4:3 ratio, the resolution has been applied to 42 -inch $(106.7 \mathrm{~cm})$ and smaller screen sizes because it was challenging to manufacture high-resolution panels in small screen sizes during the early stages of PDP development. Each pixel in a $1024 \times 768$ panel is rectangular while each pixel in a $1366 \times 768$ panel is square. (See Table 4-18) For this reason, PDP TVs larger than 50 inches $(127 \mathrm{~cm})$ have historically had $1366 \times 768$ (HD) and 1920×1080 (full HD) resolutions corresponding to a 16:9 ratio. However, a new product category of 50-inch $(127-\mathrm{cm})$ $1024 \times 768$ PDP was added in 2010, which may be an alternative to allow PDP manufacturers to meet the new energy-efficiency standards.

\subsubsection{Technology Options for Efficiency Improvement in PDP TVs}

\section{A] High Luminescence Efficiency}

Improving the efficiency of PDP TVs depends primarily on the luminescence efficiency of the PDP itself. According to the EuP Preparatory Study (Fraunhofer 2007e), the Japanese PDP manufacturers - Panasonic, Pioneer, and Hitachi - stated that a HD PDP TV with the best available technologies features a $1.8 \mathrm{~lm} / \mathrm{W}$ panel. These manufacturers expected to be manufacturing panels with an efficacy of $3 \mathrm{~lm} / \mathrm{W}$ by 2010 . The two Korean manufacturers, LG Electronics and Samsung, also stated in the same report that 2 to $3 \mathrm{~lm} / \mathrm{W}$ would be a realistic assumption for HD PDP TVs in 2010. According to experts, the luminous efficacy of current PDPs is between 2.5 and $3 \mathrm{~lm} / \mathrm{W}$. However, manufacturers have recently shifted to using luminance $\left(\mathrm{cd} / \mathrm{m}^{2}\right)$ over power consumption (W) at $20 \%$ to $30 \%$ APL as an internal efficiency index instead of conventional luminous efficacy $(\mathrm{lm} / \mathrm{W})$.

The coplanar discharge mechanism of conventional PDPs has been said to have an intrinsic upper limit of 3

63 According to ENERGY STAR Version 5 (effective in 2012), TVs larger than 1,068 in ${ }^{2}$ (corresponding to 50-inch) are required to consume no more than $108 \mathrm{~W}$ in on-mode power. 
to $5 \mathrm{~lm} / \mathrm{W}$ (Young and Chung 2007). Therefore, manufacturers have invested in developing new cell structures and materials to achieve more than $5 \mathrm{~lm} / \mathrm{W}$. These technological options are not yet available at mass production levels because a new cell design includes changes in cell structure, material composition, and driving scheme, which affect the manufacturing process, yield, and other functionalities such as device lifetime and image quality. For these reasons, it is difficult for manufacturers to anticipate the success of technology options for new cell design. Nevertheless, high-efficiency PDPs, if successfully developed, might result in better image quality and lower manufacturing cost than is currently the case for PDP TVs.

PDPs will need to overcome these existing intrinsic limitations to efficiency improvement during the next few years to reduce energy consumption and remain competitive. To improve PDP efficiency, it is very important to control the discharge mechanism associated with cell structure, materials, and gas composition.

\section{a. Improved Discharge Efficiency (Increased UV Generation Efficiency)}

\begin{tabular}{l|l}
\hline \multicolumn{1}{|c|}{ PDP Pixel } & \multicolumn{1}{c}{ PDP Cell Design Factors } \\
\hline Electrode(A) & $\begin{array}{l}\text { 1. Discharge Efficiency } \\
\text { - gas composition } \\
\text { - electron emission layer (MgO or/and new layers) } \\
\text { - cell design } \\
\text { (cell shape, electrode(A) shape, and gaps between electrodes) }\end{array}$ \\
$\begin{array}{l}\text { 2. Optical Efficiency } \\
\text { - cell design (cell aperture ratio) } \\
\text { - phosphors } \\
\text { - transmittance in electrode(A) and dielectric layer }\end{array}$
\end{tabular}

Source: manufacturer

Figure 4-19. Typical Structure of a PDP Pixel

\section{- Gas Composition}

Figure 4-19 above shows the typical structure of a PDP pixel. PDP pixels contain a combination of inert gases: xenon (Xe), neon $(\mathrm{Ne})$, and helium $(\mathrm{He})$. It is generally accepted within the industry that a high proportion of Xe can increase UV generation although this requires higher drive voltage and has associated tradeoffs with quality and reliability. Historically, manufacturers have increased the portion of Xe in the composition from $4 \%$ to $15 \%$. Although increasing the amount of Xe is still, theoretically, an efficiency improvement option, manufacturers say that there appears to be little opportunity to further increase the percentage of Xe without a significant negative impact on quality and reliability.

\section{- New Electron Emission layer}

In conventional PDPs, a magnesium oxide $(\mathrm{MgO})$ layer is used to protect the dielectric layer of the pixel and to generate secondary electron emission. Since 2007, major manufacturers have improved discharge efficiency by adding a layer to the $\mathrm{MgO}$ layer to lower discharge voltage and accelerate electron emission. Pioneer's "Crystal Emissive Layer" is one such example (Barker 2006). Much recent literature on PDP efficiency improvement focuses on new electron emission layers such as calcium oxide $(\mathrm{CaO})$ and strontium oxide $(\mathrm{SrO})$ (Khorami 2010, Whang 2010). A combination of appropriate gas composition and double protective layers, e.g., $\mathrm{CaO}-\mathrm{MgO}$ or $\mathrm{SrO}-\mathrm{MgO}$, can increase luminous efficacy by lowering drive voltage. A manufacturer says that this kind of additional layer are expected to be applied to new PDP models.

\section{- Cell Design}

Conventional PDPs consist of three electrodes: two in the front glass and one in the rear glass. The 
system, so the cell design needs to be adjusted for the change. Improvement in ERCs appears to be more technically feasible than development of low-permittivity materials.

\section{C] Improvement in Filter Transmittance}

As mentioned in Section 4.2.1, the transmittance of existing PDP filters is $40 \%$ to $48 \%$. Improvement in filter transmittance will lower the target luminance level that a bare panel needs to generate, reducing power consumption. However, because this option has trade-offs with other functionalities such as faithful color reproduction, manufacturers are unsure about the net benefit of improving filter transmittance as an option to improve overall efficiency.

The technical options for efficiency improvement discussed above are interconnected and cannot be developed in isolation. Therefore, the final efficiency improvement impacts from these options are difficult to predict accurately. Manufacturers say that a new combination of these options will be applied to 2011 PDP models that will reduce power consumption by $20 \%$ to $30 \%$ in comparison with 2010 models. Panasonic claims that the 2011 Panasonic 42-inch $(106.7-\mathrm{cm})$ FHD PDP TVs will consume half the power of 2009 PDP TVs because of the development of new phosphors, advanced cell structure with an optimized transparent electrode, slimmer barrier ribs, and a high-speed driving scheme.

\subsubsection{Cost Reduction due to Super-Efficient PDPs}

Although some options exist to improve the efficiency of PDPs, most options involve increased R\&D expenditures or a large-scale change in the manufacturing line. Moreover, some options require or affect proprietary technology. Therefore, it is hard to predict incremental costs for energy-efficiency improvements in panel technologies and also hard to extrapolate these incremental costs across TV models and manufacturers. However, panel efficiency improvements are also likely to result in cost savings because of lower materials costs.

In 2007, DisplaySearch analyzed the relationship between efficiency improvement and cost reduction in PDPs (Young and Chung 2007). According to the DisplaySearch analysis, if 42-inch HD PDPs can achieve an efficiency of $5 \mathrm{~lm} / \mathrm{W}$, the total manufacturing cost would decrease by $9 \%$ to $11 \%$ compared to the costs for the current $2.5 \mathrm{~lm} / \mathrm{W}$ (See Figure 4-21) because the high efficiency would allow the panel to use low drive voltage and have simplified heat solutions as well as lower power supply costs. For $10 \mathrm{~lm} / \mathrm{W}$ PDPs, the DisplaySearch analysis described new discharge models that some manufacturers had studied. Although innovative PDP cell structures have been expected to significantly lower production costs by reducing numerous manufacturing steps as well as material costs, the realization of those changes is uncertain.

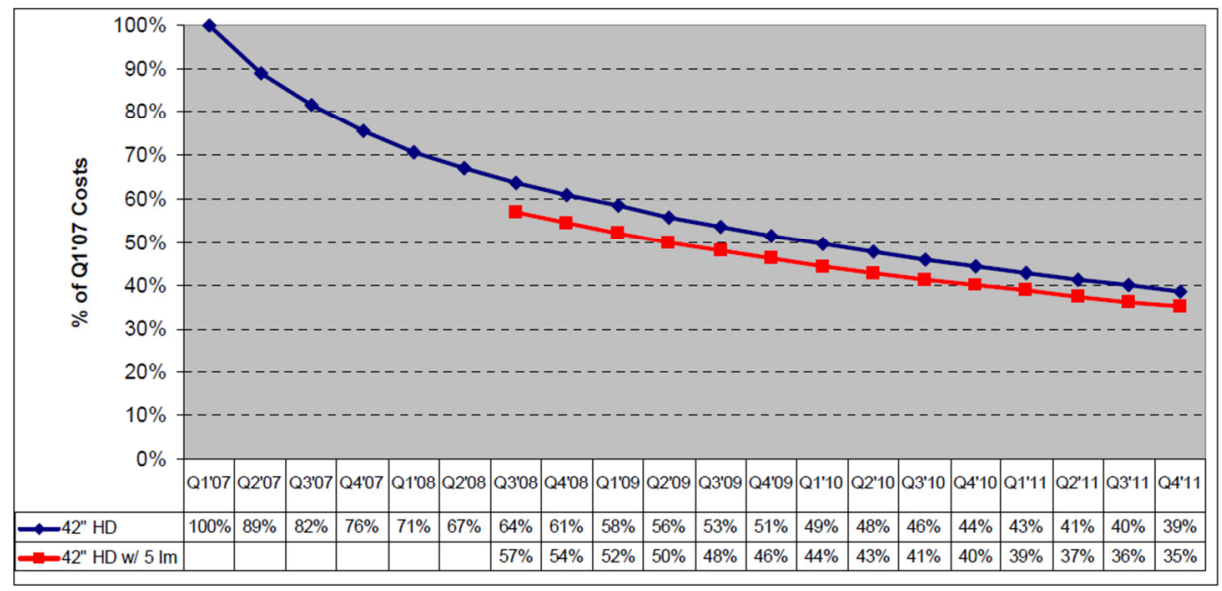

Source: Young and Chung 2007

Figure 4-21. Indexed 42-inch HD Plasma Panel Costs at $2.5 \mathrm{~lm} / \mathrm{W}$ and $5.0 \mathrm{~lm} / \mathrm{W}$ 


\subsubsection{Summary of Efficiency Improvement Options in PDP TVs}

Although further efficiency improvements in PDP TVs are likely, improvement to the super-efficiency levels that LED backlit LCDs are forecast to achieve is uncertain for PDP TVs for three reasons. First, PDP panel improvement would be the key development to improve energy efficiency, but the technologies needed to improve PDP panel efficiency require large-scale and interconnected changes in design and manufacturing of PDPs; therefore, the efficiency improvement potential from any one option or combination of options is difficult to isolate and predict accurately. Second, although improvement of filter transmittance may be possible, it has trade-offs with other functionalities such as color reproduction. Last, PDP market share is not very significant compared to that of LCD TVs, and PDPs are positioned in the large (over 40-inch) screen market. Therefore, we conclude that currently envisioned and forthcoming efficiency standards will likely be sufficient to realize the currently available market potential for improved efficiency of PDPs.

Based on the roadmap of major manufacturer roadmaps, efficient 42-inch to 50-inch PDPs are expected to qualify for ENERGY STAR Version 5 in 2012. In fact, five recent Panasonic PDP TV models have qualified for ENERGY STAR Version 5. (ENERGY STAR 2011) However, it is uncertain whether PDP TVs over 50 inches will meet the most stringent standard, $108 \mathrm{~W}$. The average on-mode power of 54-inch Panasonic PDP TVs in March 2011 is about $160 \mathrm{~W}$, that of 58-59-inch Panasonic and Samsung PDP TVs is about $180 \mathrm{~W}$, and that of 63-65-inch is about $210 \mathrm{~W}$. Additional solutions are required for those sizes to meet the new standard. Figure 4-22 shows the on-mode power forecast for 42-inch, 1080p PDP TVs.

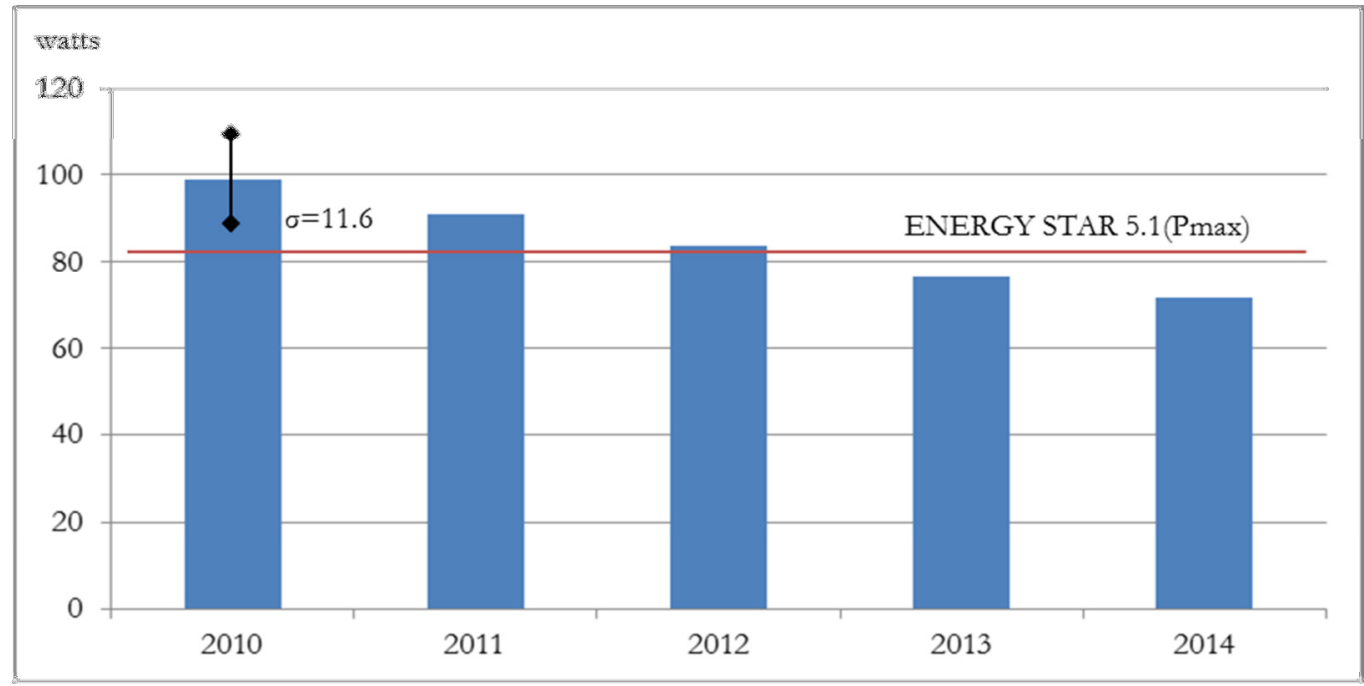

\begin{tabular}{|c|c|}
\hline $\begin{array}{l}\text { Improvernen: } \\
\text { Options }\end{array}$ & 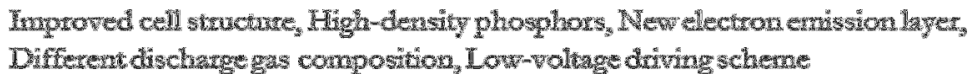 \\
\hline
\end{tabular}

Figure 4-22. Forecast for On-Mode Power Consumption of 42-inch PDP TVs 


\subsection{OLED TV}

Since 2006, OLEDs have been rapidly growing in small-size mobile applications, and major TV brands are now introducing OLED TVs. In 2007, the first OLED TV, the Sony 11-inch (27.9-cm) XEL-1 was introduced to the market, and LG Electronics launched its first OLED TV model, the 15-inch $(38.1-\mathrm{cm})$ EL9500 in 2010. Sony's 27-inch (68.6-cm), Samsung's 30-inch (76.2-cm) and 40-inch (101.6-cm), and LG's 31inch $(78.7-\mathrm{cm})$ OLED TV prototypes have been demonstrated in recent exhibitions (Young 2009). It is expected that medium- to large-size (i.e., larger than 30 inches) OLED TVs will be commercially available in late 2011 or early 2012.

\subsubsection{Power Consumption of OLED TVs}

OLEDs have a great advantage over LCDs in terms of power consumption because each pixel in an OLED is individually controlled to generate light according to input signal images. However, there are few data regarding actual power consumption for large OLED TVs. According to ENERGY STAR, the on-mode power consumption of the Sony 11-inch XEL-1 was 26 W. (IEA 2010e) According to experts, the model consumes $25 \mathrm{~W}$ in full white mode $\left(180 \mathrm{~cd} / \mathrm{m}^{2}\right)$, of which the panel accounts for $9 \mathrm{~W}$ to $10 \mathrm{~W}$. This indicates that basic power consumption, including digital signal processing, is about $15 \mathrm{~W}$ to $16 \mathrm{~W}$ (i.e., in full black mode). According to manufacturers, a 30- to 32 -inch $(76.2-\mathrm{cm})$ OLED TV panel consumes about $30 \mathrm{~W}$ in full white mode (at $\left.200 \mathrm{~cd} / \mathrm{m}^{2}\right)$. The on-mode power consumption of the TV set is expected to be $15 \mathrm{~W}^{65}$ Assuming the basic power consumption including digital signal processing is $18 \mathrm{~W}$ to $25 \mathrm{~W}$ at on-mode, the on-mode power consumption of the OLED TV set is expected to be about $33 \mathrm{~W}$ to $40 \mathrm{~W}$ in normal video mode. Table 4-20 shows OLED TV estimated on-mode power consumption.

\begin{tabular}{l|c|c|c}
\hline \multicolumn{1}{c|}{ Screen Size } & Screen Area & Resolution & $\begin{array}{c}\text { On-Mode Power } \\
\text { (Estimated) }\end{array}$ \\
\hline $\begin{array}{l}\text { 11-inch }(27.9-\mathrm{cm}) \\
\text { (commercialized in 2007) }\end{array}$ & $\begin{array}{c}51 \mathrm{inch}^{2} \\
\left(333 \mathrm{~cm}^{2}\right)\end{array}$ & $960 \times 540$ pixels & $25-26 \mathrm{~W}$ \\
\hline $\begin{array}{l}\text { 30- to 32-inch } \\
(76.2 \text { - to } 81.3 \text {-cm) } \\
\text { (prototype in 2010) }\end{array}$ & $\begin{array}{c}384 \text { to } 437 \text { inch }^{2} \\
\left(2,479 \text { to } 2,822 \mathrm{~cm}^{2}\right)\end{array}$ & $1920 \times 1080$ pixels & $33-40 \mathrm{~W}$ \\
\hline
\end{tabular}

\section{Table 4-20. Estimated On-mode Power Consumption of OLED TVs}

\subsubsection{Technology Options for Efficiency Improvements in OLED TVs}

The average luminous efficacy (50 to $60 \mathrm{~lm} / \mathrm{W}$ ) of OLEDs currently on the market is lower than that of LEDs (70 to $100 \mathrm{~lm} / \mathrm{W}$ ), but OLED TVs would be more efficient than LED backlit LCD TVs because the total efficiency of an LCD TV is below 10\% from backlight to screen, as described in Section 3.2.1, as a result of low panel transmittance. As mentioned above, a 30-inch $(76.2-\mathrm{cm})$ OLED TV module with $1920 \times 1080$ pixels consumes about $30 \mathrm{~W}$. According to the manufacturer, the OLED materials account for $60 \%$ to $70 \%$ of the power consumption; the rest is for the TFT drive. The performances of light-emitting materials and TFTs are the key factors in OLED TV efficiency improvement.

\section{A] Materials Improvement (PHOLED)}

According to Samsung, phosphorescent light-emitting materials are being improved and will replace

65 In general, the power consumption required for normal video images should be about $20 \%$ of the full-white screen at peak luminance. However, since our estimation is not based on power at peak luminance $\left(500\right.$ to $\left.600 \mathrm{~cd} / \mathrm{m}^{2}\right)$ but on power at normal brightness $\left(200 \mathrm{~cd} / \mathrm{m}^{2}\right)$, the on-mode power is assumed to be half of the power at the normal full white mode, based on the results from the Sony 11-inch XEL-1. 
fluorescent emitters, resulting in greater power efficiency. Samsung (Kim et al. 2009) reported Universal Display Corp's claim that a 40-inch (101.6-cm) OLED TV panel based on phosphorescent OLED (PHOLED) can consume less than $15 \mathrm{~W}$ at $300 \mathrm{~cd} / \mathrm{m}^{2}$. Figure $4-23$ shows consumption for different types of PHOLED TV panels.

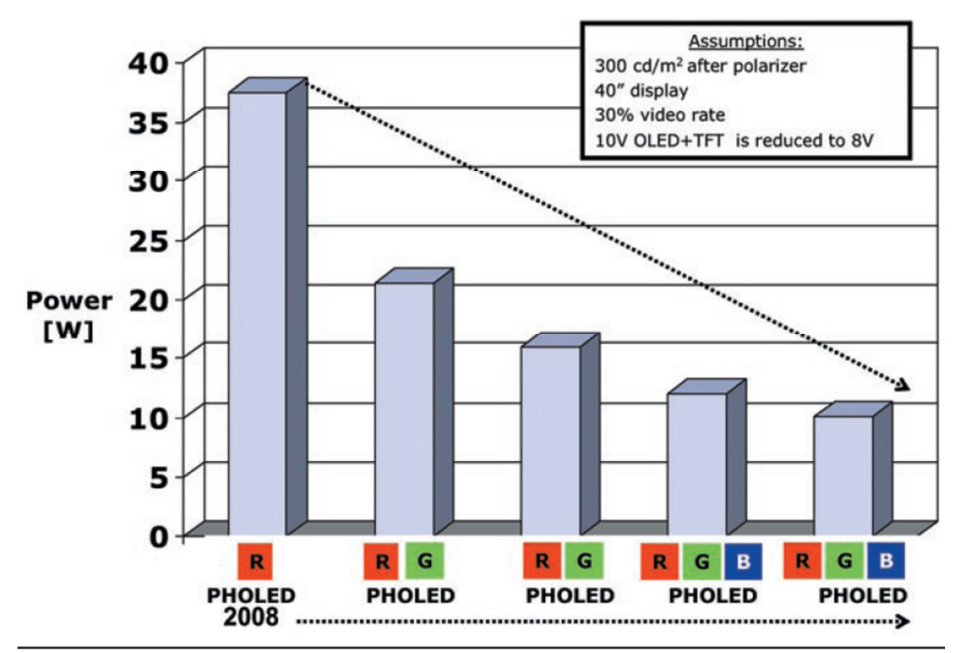

Source: Kim et al. 2009

\section{Figure 4-23. Power Consumption Roadmap for 40-inch (101.6-cm) OLED TV Panels}

Phosphorescent materials are known to be about four times better than fluorescent materials in "internal quantum efficiency," ${ }^{66}$ i.e., almost 100\%. However their emission mechanism is not stable, and phosphorescent emission is slower than fluorescent emission. In addition, development of a blue phosphor is important for OLED efficiency improvement. Blue plays the most significant role in the overall colorreproduction capability of OLEDs. If a display wants to achieve "deep blue" at a lower position in the y-axis of color coordinates for better color gamut, the blue phosphors used will consume more power. In fact, blue materials (fluorescent/phosphorescent) have the lowest efficacy levels in candelas per ampere (cd/A) (OLED Association 2010) According to experts, Sony's 11-inch XEL-1 panel consumes $6 \mathrm{~W}$ in full blue mode, 2.5 $\mathrm{W}$ in full green mode, $4 \mathrm{~W}$ in full red mode, and $9 \mathrm{~W}$ in full white mode. Manufacturers also mentioned that trade-offs between the blue color coordinate and efficiency have been an issue in development of OLED displays, rather than other colors. In other words, while manufacturers want to achieve "deep blue" color in their displays, located at lower y-axis in the CIE color (chromaticity) diagram (Figure 4-24), efficiency of lighting materials with "deep blue" color is lower than other materials whose blue color coordinate is located higher than theirs.

66 In theory, an OLED's power efficiency is calculated by multiplying internal quantum efficiency, external quantum efficiency, and other factors. Internal quantum efficiency (IQE) is defined as the fraction of neutral excited states, and external quantum efficiency (EQE) as the fraction of emitted photons that are coupled out of the device. It is said that max EQE is about 20\% and IQE is about $100 \%$. 


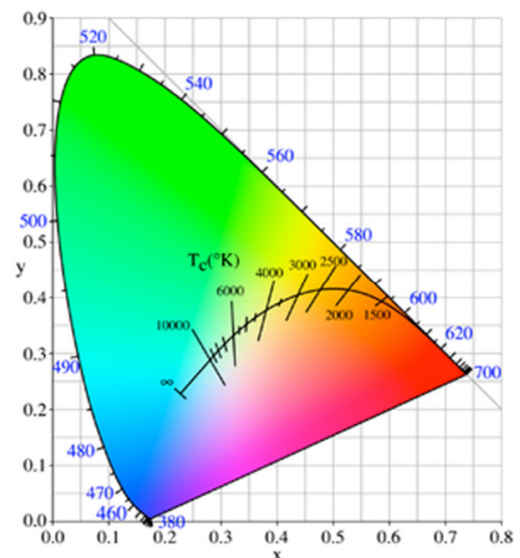

Figure 4-24. CIE Chromaticity Diagram

\section{B] Thin-Film 'Transistor (TFT') Backplane}

Manufacturers report that there have been many studies on improving TFT mobility, which can increase the TFT drive's efficiency. However, this technology option is not fully developed yet because of issues such as lifetime. Oxide-based semiconductors have recently been widely investigated as a solution because of their high mobility, high transparency, low processing temperature, and potentially good uniformity. (Hsieh 2010) Many researchers are interested in oxide TFTs because they have higher mobility than amorphous silicon TFTs and better uniformity than low-temperature polycrystalline silicon TFTs. Manufacturers see oxide TFTs as strong candidates to improve the overall quality of OLEDs.

Although OLEDs have energy-efficiency improvement potential, the options are strongly tied into proprietary OLED panel technologies, including a sophisticated manufacturing process. Therefore, it is difficult to estimate incremental costs and $R \& D$ investments corresponding to this efficiency improvement potential. However, we forecasted an average on-mode power of 32-inch OLED TVs based on the above discussion. Figure 4-25 shows this on-mode power forecast.

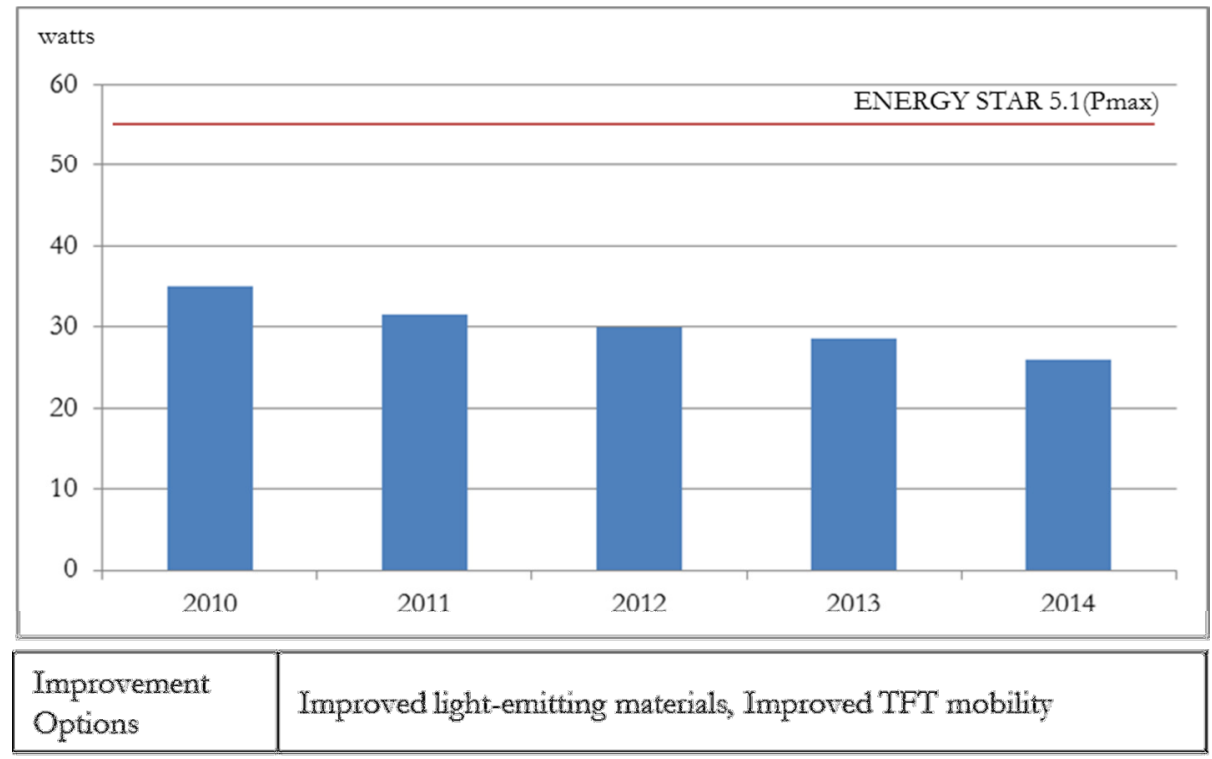

Figure 4-25. Forecast for On-Mode Power of a 32-inch OLED TV 


\subsubsection{Comparison of Production Cost: OLED T'V and LCD TV}

So far, commercially available OLED TV models are the Sony XEL-1 (11-inch or $27.9-\mathrm{cm}, 960 \times 540$ pixels) and LG EL9500 (15-inch or 38.1-cm, 1366×768 pixels). OLED TVs larger than 30 inches $(76.2 \mathrm{~cm})$ are expected to be commercially available in 2012. Because OLED TVs are an emerging technology, it is difficult to predict manufacturing cost at full scale. In addition, most efficiency improvement options depend on proprietary OLED panel technologies.

Because there is little history of OLED TV cost modeling, the current DisplaySearch forecast for OLED TVs is based on the history of LCD TVs. Although the OLED TV cost and price forecast will become more reliable when large-size OLED TVs are realized in the market, it might be useful to consider currently expected price gaps between OLED TVs and LCD TVs. As mentioned in Section 2.1.3., a recent DisplaySearch report (DisplaySearch 2011f) expected that 40- to 55-inch OLED TVs will be commercialized from the year 2012 forward, while the previous reports from DisplaySearch forecasted that 32- to 40-inch OLED TVs would be commercialized first in 2012. Because of cost-competitiveness and market positioning, manufacturers will be likely to provide OLED TVs over 40 inches for flagship models.

The average market price of 40- to 55-inch (101.6- to 139.7-cm) OLED TVs is expected to be about 2.5 times that of LED backlit TVs in 2014, as shown in Figure 4-26. According to the report, the reseller margin for those OLED TVs was assumed to be $29 \%$ to $34 \%$, and the brand margin was assumed to be $2 \%$ to $6 \%$ during the period. The reseller margin for the same size LED-LCD TVs was estimated at 11 to 14\%, and the brand margin was estimated at $-2 \%$ to $3 \%$ for the same period. Although it does not appear that OLED TVs will be a cost effective technology in the short term, the production efficiency, or costs, are expected to be improved as the number of products being produced increases. The uncertainty in costs is yet too great to make recommendations regarding a potential market transformation program for energy-efficiency improvement of OLED TVs.

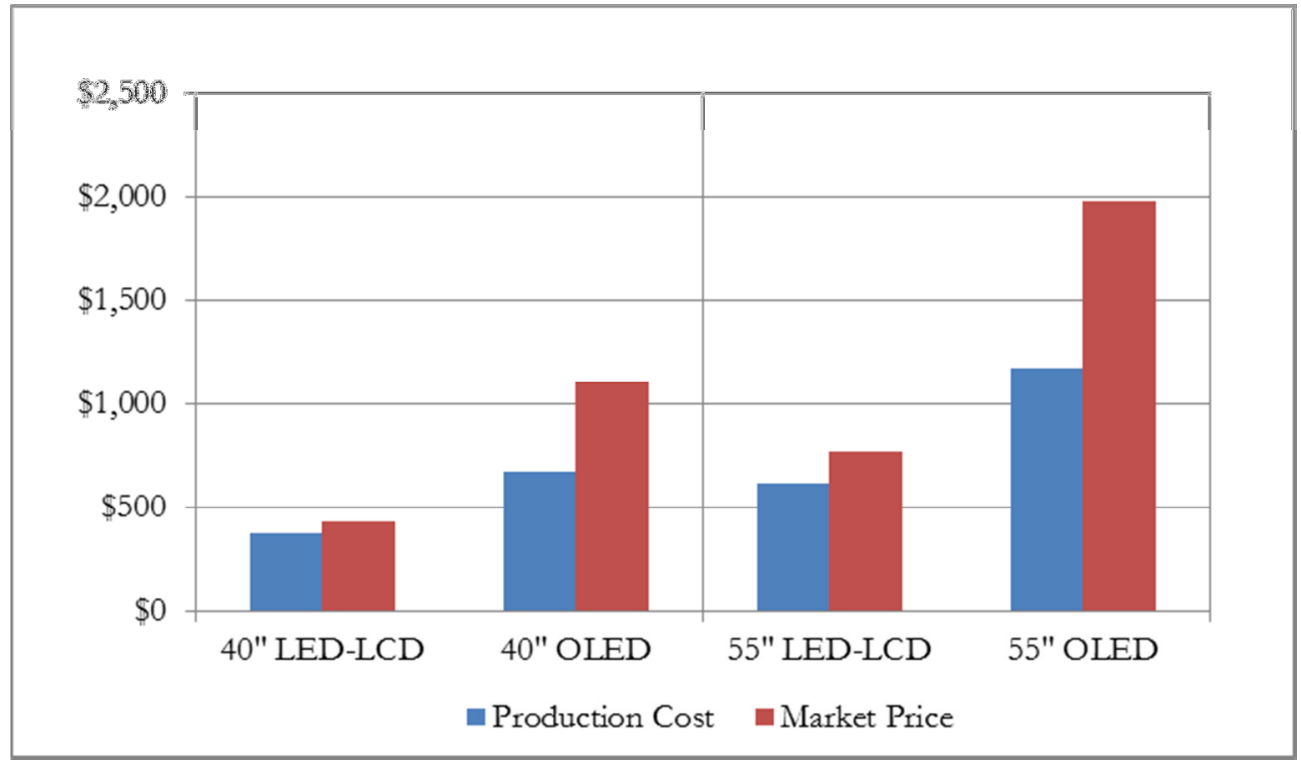

Source: DisplaySearch $2010 \mathrm{~g}$

Figure 4-26. Forecast OLED TV Production Cost and Average Market Price 


\section{Estimates of Energy Savings Potentials (BUENAS)}

The Bottom Up Energy Analysis System (BUENAS) is an end-use energy forecasting model developed by Lawrence Berkeley National Laboratory (LBNL) in the United States and supported by the Collaborative Labeling and Appliance Standards Program (CLASP). Its original goal was to provide a more detailed and accurate assessment of the potential for energy savings and greenhouse gas emissions reductions from energy-efficiency standards and labeling programs worldwide. BUENAS is used to provide an estimate of energy savings that would result from the SEAD initiative. The following section describes BUENAS, the data and assumptions used for our analysis, and the preliminary results.

\subsection{Description of BUENAS}

BUENAS is a "bottom up" model that calculates energy demand for appliance types based on input data for individual product types. It uses a basic activity/intensity approach, first calculating the number of a given appliance in each country in each year (Module 1) and then multiplying by unit energy consumption in each scenario (Module 2). A third module calculates the impact of efficiency programs on the national stock of appliances by tracking sales and retirements. Figure 5-1 shows the structure of the BUENAS analysis.

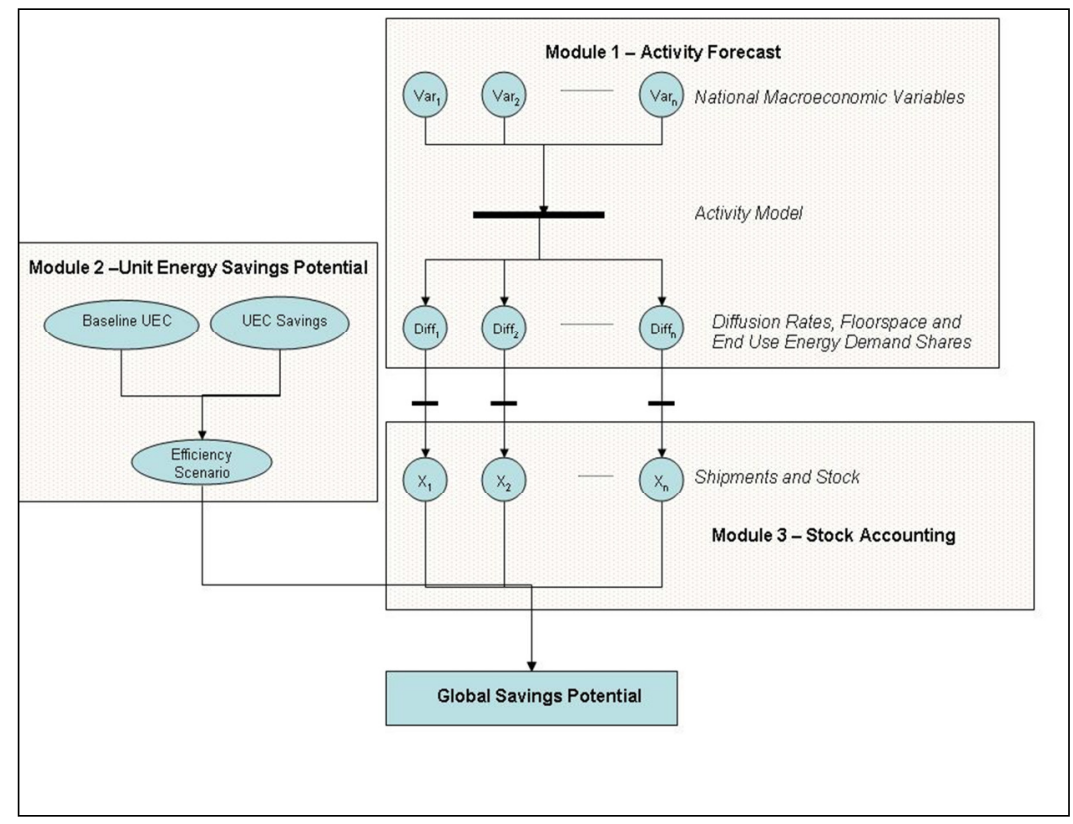

Source: McNeil et al. 2008

Figure 5-1. BUENAS Analysis Structure

BUENAS inputs are product ownership rates, product sales, annual unit energy consumption, and per-unit percentage improvement potential. In the absence of reliable market data, BUENAS forecasts appliance stock and sales using an econometric model based on household income, gross domestic product, and population.

BUENAS is implemented using the Long-Range Energy Alternatives Planning system, developed by the Stockholm Environment Institute. This system is a general-purpose energy accounting model in which the model developer inputs all data and assumptions in a format that is transparent to other users. Using this platform, BUENAS can be easily shared for review and collaboration, including cross-referencing with existing national models. BUENAS may also incorporate data and assumptions developed by technical teams from SEAD-14. 


\subsection{Description of Data Inputs}

Each SEAD-14 country was modeled separately in BUENAS. The detailed country and appliance data are available in Appendix B.

\subsubsection{TV Shipment/Sales}

TV shipment data and forecast are available in DisplaySearch report (2010b, 2011a) from 2007 to 2014. To estimate future savings for the years 2015, 2020 and 2030, sales have to be forecast. We used the sales forecast from McNeil et al. (2008) based on a macroeconomic model in order to determine the growth rate of sales after 2014. We used data from Letschert (2009) for China. The growth rates from DisplaySearch do not appear to be sustainable after 2014, so they are shown here as indicative. Table 5-1 summarizes the TV shipment/sales data inputs.

\begin{tabular}{|c|c|c|c|c|c|}
\hline Country & $\begin{array}{l}\text { Shipment } \\
\text { or Sales } \\
\text { in } 2007 \\
\text { (Millions) }\end{array}$ & $\begin{array}{l}\text { Shipment } \\
\text { or Sales } \\
\text { in } 2014 \\
\text { (Millions) }\end{array}$ & Additional Assumptions & $\begin{array}{l}\text { Growth Rate } \\
\text { Based on } \\
\text { Macroeconomic } \\
\text { Model 2014- } \\
2030\end{array}$ & $\begin{array}{l}\text { Growth } \\
\text { Rate Based } \\
\text { on Display } \\
\text { Search Data } \\
\text { 2007-2014 }\end{array}$ \\
\hline Australia & 7.7 & 9.9 & $20 \%$ of Asia Pacific & $4 \%$ & $4 \%$ \\
\hline Brazil & 10.7 & 12.8 & - & $2 \%$ & $3 \%$ \\
\hline Canada & 3.8 & 5.8 & $11 \%$ of North America & $1 \%$ & $6 \%$ \\
\hline China & 39.6 & 60.0 & - & $1 \%$ & $6 \%$ \\
\hline Europe & 38.1 & 58.9 & $\begin{array}{l}\text { Western Europe + Eastern Europe - } \\
\text { Russia }\end{array}$ & $2 \%$ & $7 \%$ \\
\hline India & 12.2 & 17.4 & - & $6 \%$ & $5 \%$ \\
\hline Japan & 9.3 & 8.5 & - & $0 \%$ & $-0.01 \%$ \\
\hline Korea & 6.1 & 7.9 & $16 \%$ of Asia Pacific & $2 \%$ & $4 \%$ \\
\hline Mexico & 5.9 & 6.9 & $24 \%$ of Latin America & $2 \%$ & $2 \%$ \\
\hline Russia & 5.3 & 9.4 & $45 \%$ of Eastern Europe & $2 \%$ & $7 \%$ \\
\hline $\begin{array}{l}\text { South } \\
\text { Africa }\end{array}$ & 0.9 & 1.7 & $9 \%$ of Middle East and Africa & $2 \%$ & $9 \%$ \\
\hline U.S. & 31.1 & 46.7 & $89 \%$ of North America Market & $1 \%$ & $6 \%$ \\
\hline
\end{tabular}

Source: McNeil et al. (2008), Letschert, (2009), DisplaySearch (2010b, 2011a) and interviews with marketing experts from the industry

Table 5-1. TV Shipment/Sales by Country and Growth Rates ${ }^{67}$

\subsubsection{TV Market Share}

Our analysis divides TVs into three product classes:

- Product Class 1: CRTs

- Product Class 2: LCDs and OLEDs

- Product Class 3: PDPs

Market share data for 2007 to 2014 are available from DisplaySearch (2010b, 2011a). When forecasting

67 As discussed in Section 2.2.2., the TV data from 2007 to 2014 for Canada, China, India, Japan, and the US are for TV shipments; the data for other countries are for TV $\underline{\text { sales. }}$. 
market shares after 2014, we hold the ratio of LCD to PDP constant and forecast the remainder of the market using the 2007-2014 trend. CRTs will definitely be phased out of the international market by 2016. Figure 5-2 represents the combination of sales and market shares for the countries studied in this report.

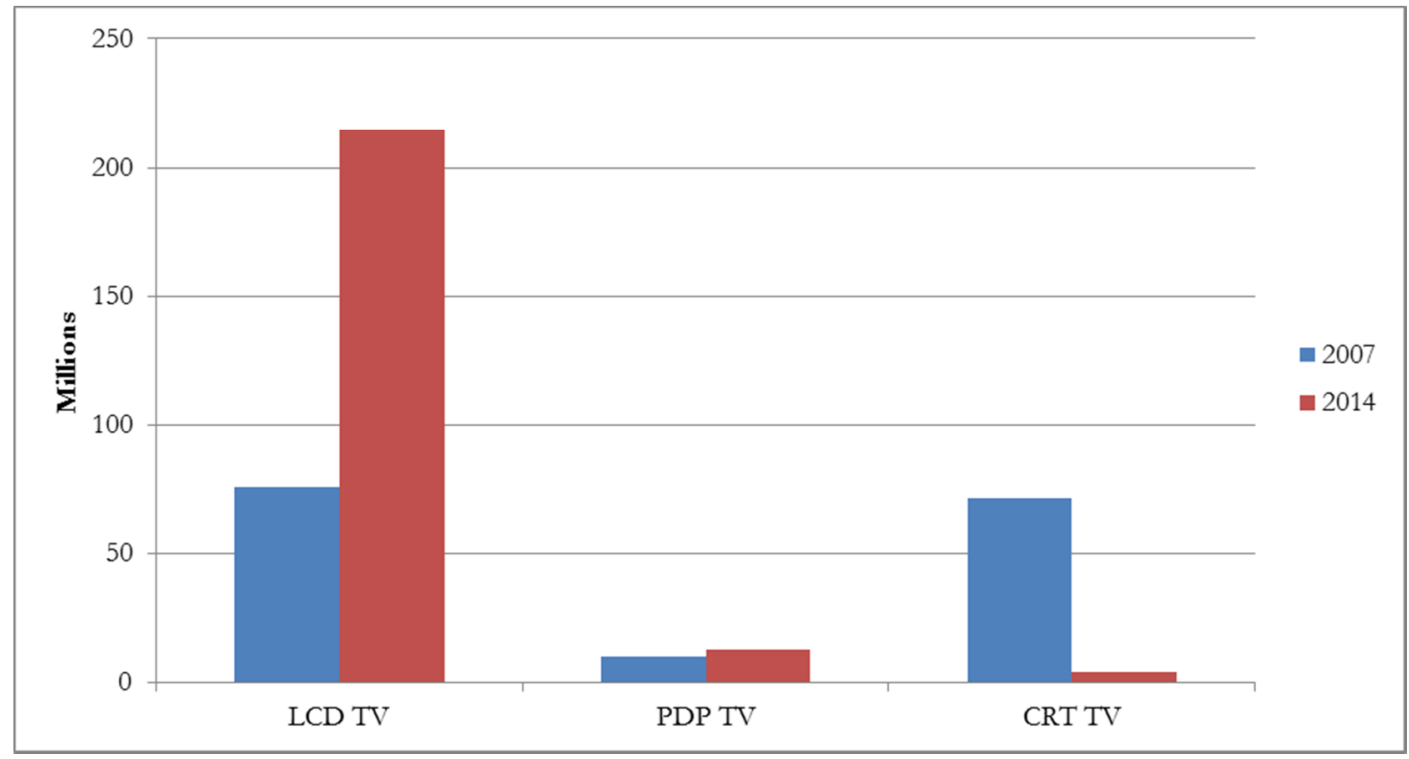

* OLED sales will be about 1 million in 2014.

\section{Figure 5-2. Global Sales of Televisions by Product Class}

\subsubsection{Base-Case Efficiency Scenario}

In the base case, we introduce the following technology options, as described in the previous section 4.1:

- LCDs with LED backlight unit

- LCDs with CCFL backlight unit (All other options but LED efficiency improvement can be applied to LCDs with CCFL backlight unit)

Table 5-2 summarizes the global market shares for each base-case technology. Different market shares and EEIs are applied to each country in the analysis. More data and references on EEI and market share assumptions can be found in Appendix B.

\begin{tabular}{c|c|c|c|c|c|c}
\hline & \multicolumn{2}{|c|}{2010} & \multicolumn{2}{c|}{2012} & \multicolumn{2}{c}{2014} \\
\hline \hline & Market Share & EEI & Market Share & EEI & Market Share & EEI \\
\hline CCFL & $80 \%$ & 0.467 & $29 \%$ & 0.401 & $12 \%$ & 0.322 \\
\hline LED & $20 \%$ & 0.356 & $71 \%$ & 0.292 & $88 \%$ & 0.239 \\
\hline
\end{tabular}

EEI (Energy Efficiency Index $)=($ on-mode power $) \div \mathrm{P}_{\max }$

$P_{\max }=4.3224 \times \mathrm{A}+20\left(\mathrm{~A}: \mathrm{dm}^{2}\right)$

Table 5-2. Efficiency and Global Market Shares by Technology in the Base Case

\subsubsection{Efficiency Scenario}

We only studied LCDs in our efficiency scenario because it is not possible to isolate cost-effective options from future plasma TV technologies with certainty. OLED TVs are incorporated as an efficiency case in the LCD scenario. CRTs will be phased out in a few years, so we did not consider this technology.

In the efficiency case, we introduce two additional levels of efficiency that include DBEF and dimming options: 
- LCDs with efficient CCFL and a global average EEI of 0.345 in 2012; this design would be $14 \%$ to $16 \%$ more efficient than the baseline CCFL.

- LCDs with efficient LED BLU and a global average EEI of 0.256 in 2012; this design would be 35\% to $36 \%$ more efficient than the baseline CCFL. Prism-patterned LGP is assumed to be employed after 2013.

In the efficiency scenario, we assume that these efficient technologies for CCFL and LED LCDs can enter the market starting in 2012. In every year the efficient designs reach $30 \%$ of the market shares for each technology type. In other words, in the efficient scenario, 30\% of CCFL-LCDs are efficient CCFL-LCDs and $30 \%$ of LED-LCDs are efficient LED-LCDs.

Different market shares and EEIs are applied to each country in the analysis. More country data on EEI assumptions can be found in the Appendix B.

Table 5-3 summarizes the market shares used in BUENAS for each technology in the efficiency scenario. More data on market share assumptions can be found in Appendix B.

\begin{tabular}{l|c|c|c|c|c|c}
\hline & \multicolumn{2}{|c|}{2010} & \multicolumn{2}{c|}{2012} & \multicolumn{2}{c}{2014} \\
\hline \hline & Market Share & EEI & Market Share & EEI & Market Share & EEI \\
\hline CCFL & $80 \%$ & 0.467 & $20.3 \%$ & 0.401 & $8.4 \%$ & 0.322 \\
\hline CCFL Eff & - & - & $8.7 \%$ & 0.345 & $3.6 \%$ & 0.280 \\
\hline LED & $20 \%$ & 0.356 & $49.7 \%$ & 0.292 & $61.6 \%$ & 0.239 \\
\hline LED Eff & - & - & $21.3 \%$ & 0.256 & $26.4 \%$ & 0.207 \\
\hline
\end{tabular}

Table 5-3. Efficiency and Global Market Shares by Technology in the Efficiency Scenario

By combining the technology market shares and specific efficiency forecasts, we estimate an average improvement of $10 \%$ of the consumption over the base case in 2012. Due to the rapidly moving baseline, a re-evaluation of the efficient target has to be done on a regular basis. We assume that after 2015, half of the 2012 incremental efficiency improvement, 5\% will be possible. We maintain this Unit Energy Consumption (UEC) improvement constant in every year of the forecast.

The following table summarizes the unit energy consumption values that were implemented into BUENAS. There is no efficiency scenario for PDP and CRTs, UECs are given as indicative of the total TV stock consumption. 


\begin{tabular}{|c|c|c|c|c|c|c|c|c|c|}
\hline \multirow{3}{*}{ Country } & \multirow[b]{2}{*}{$\begin{array}{l}\text { Hours of } \\
\text { Usage per } \\
\text { Day }\end{array}$} & \multicolumn{4}{|l|}{ LCD } & \multicolumn{2}{|l|}{ PDP } & \multicolumn{2}{|l|}{ CRT } \\
\hline & & $\begin{array}{l}\text { Base Case } \\
\text { UEC } \\
2012\end{array}$ & $\begin{array}{l}\text { Efficiency } \\
\text { Case UEC } \\
2012\end{array}$ & $\begin{array}{l}\text { Base Case } \\
\text { UEC } 2015\end{array}$ & $\begin{array}{l}\text { Efficiency } \\
\text { Case UEC } \\
2015\end{array}$ & $\begin{array}{l}\text { Base } \\
\text { Case } \\
\text { UEC } \\
2012\end{array}$ & $\begin{array}{l}\text { Base } \\
\text { Case } \\
\text { UEC } \\
2015\end{array}$ & $\begin{array}{l}\text { Base } \\
\text { Case } \\
\text { UEC } \\
2012\end{array}$ & $\begin{array}{l}\text { Base } \\
\text { Case } \\
\text { UEC } \\
2015\end{array}$ \\
\hline & hrs & $\mathrm{kWh} / \mathrm{yr}$ & $\mathrm{kWh} / \mathrm{yr}$ & $\mathrm{kWh} / \mathrm{yr}$ & $\mathrm{kWh} / \mathrm{yr}$ & $\mathrm{kWh} / \mathrm{yr}$ & $\mathrm{kWh} / \mathrm{yr}$ & $\mathrm{kWh} / \mathrm{yr}$ & $\mathrm{kWh} / \mathrm{yr}$ \\
\hline Australia & 6.5 & 111.5 & 107.1 & 82.3 & 75.2 & 237.1 & 202.0 & 110.8 & 109.2 \\
\hline Brazil & 4 & 70.2 & 67.2 & 52.5 & 50.0 & 143.7 & 123.8 & 70.9 & 69.9 \\
\hline Canada & 4.5 & 90.0 & 86.6 & 75.7 & 69.3 & 190.5 & 171.4 & & \\
\hline China & 4 & 76.3 & 73.3 & 57.4 & 52.5 & 145.3 & 126.2 & 74.2 & 73.1 \\
\hline Europe & 4 & 64.4 & 62.0 & 52.0 & 47.5 & 146.5 & 126.7 & & \\
\hline India & 3.5 & 60.1 & 57.7 & 45.9 & 41.9 & 127.7 & 108.7 & 59.7 & 58.8 \\
\hline Japan & 4.5 & 73.2 & 70.6 & 60.0 & 54.9 & 158.0 & 138.6 & & \\
\hline Korea & 6 & 103.0 & 98.9 & 76.0 & 69.4 & 218.9 & 186.4 & 102.3 & 100.8 \\
\hline Mexico & 4 & 70.1 & 67.0 & 52.4 & 47.5 & 136.7 & 113.8 & 70.9 & 69.9 \\
\hline Russia & 4 & 68.7 & 66.0 & 52.9 & 48.4 & 140.1 & 120.2 & 71.1 & 71.1 \\
\hline South Africa & 4 & 70.2 & 67.4 & 51.6 & 47.2 & 141.6 & 122.7 & 69.8 & 68.8 \\
\hline US & 5 & 100.0 & 96.2 & 84.1 & 77.0 & 211.6 & 190.4 & & \\
\hline
\end{tabular}

Notes: In order to simplify the forecast, UECs are maintained constant after 2015, because we only calculate savings. OLEDs are folded into LCD UECs.

\section{Table 5-4. TV Usage and Energy Consumption by Country}

The following figure (Figure 5-3) presents a forecast for the energy consumption of TVs in the selected countries by TV type in the BAU. Because of the rapidly evolving nature of the technologies, the figures after 2015 must be understood as indicative. TV electricity consumption is expected to slightly decrease in the short term, because of a large-scale technological transition (e.g., CRT to LCD, and CCFL-LCD to LEDLCD) and rapid improvements in TV energy efficiency, in spite of the projected increase in penetration of TVs in households, especially in emerging economies, as well as the projected increase in the average screen size of TVs purchased. Figure 5-4 shows the forecast for energy consumption of TVs in selected countries. The selected countries represent more than $85 \%$ of the global TV market. According to the forecast, TVs are estimated to consume about 168 TWh in 2010. Given that total global electricity consumption is about 5,000 $\mathrm{TWh}^{68}$, the energy consumed by TVs in 2010 accounts for $3 \%$ to $4 \%$.

68 According to U.S. DOE International Energy Outlook 2010 (DOE 2010c), total electricity consumption in residential sector was 15.8 Quadrillion (Quad) Btu, equivalent to 4,631 TWh, in 2007 and forecasted 19.2 Quad Btu, equivalent to 5,627 TWh, in 2015. 


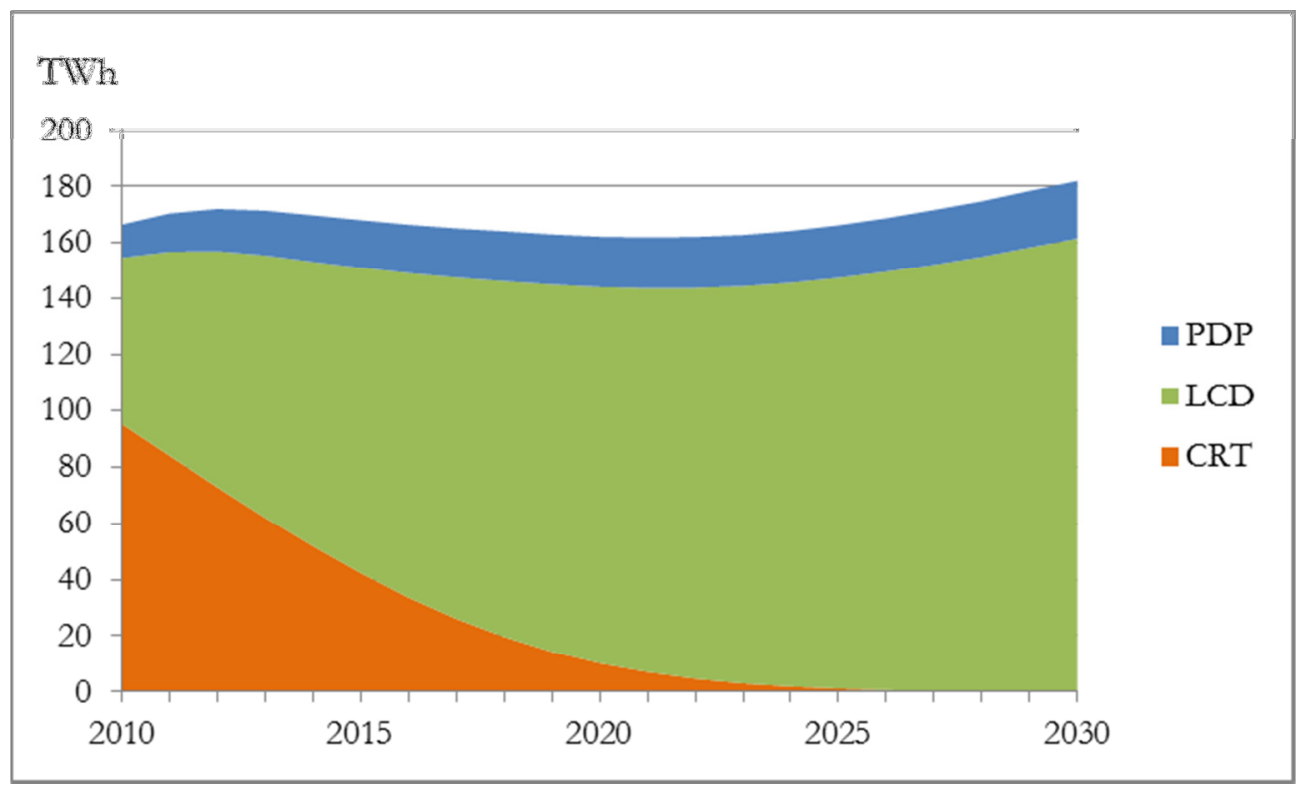

Figure 5-3. Global Consumption of TVs by Product class

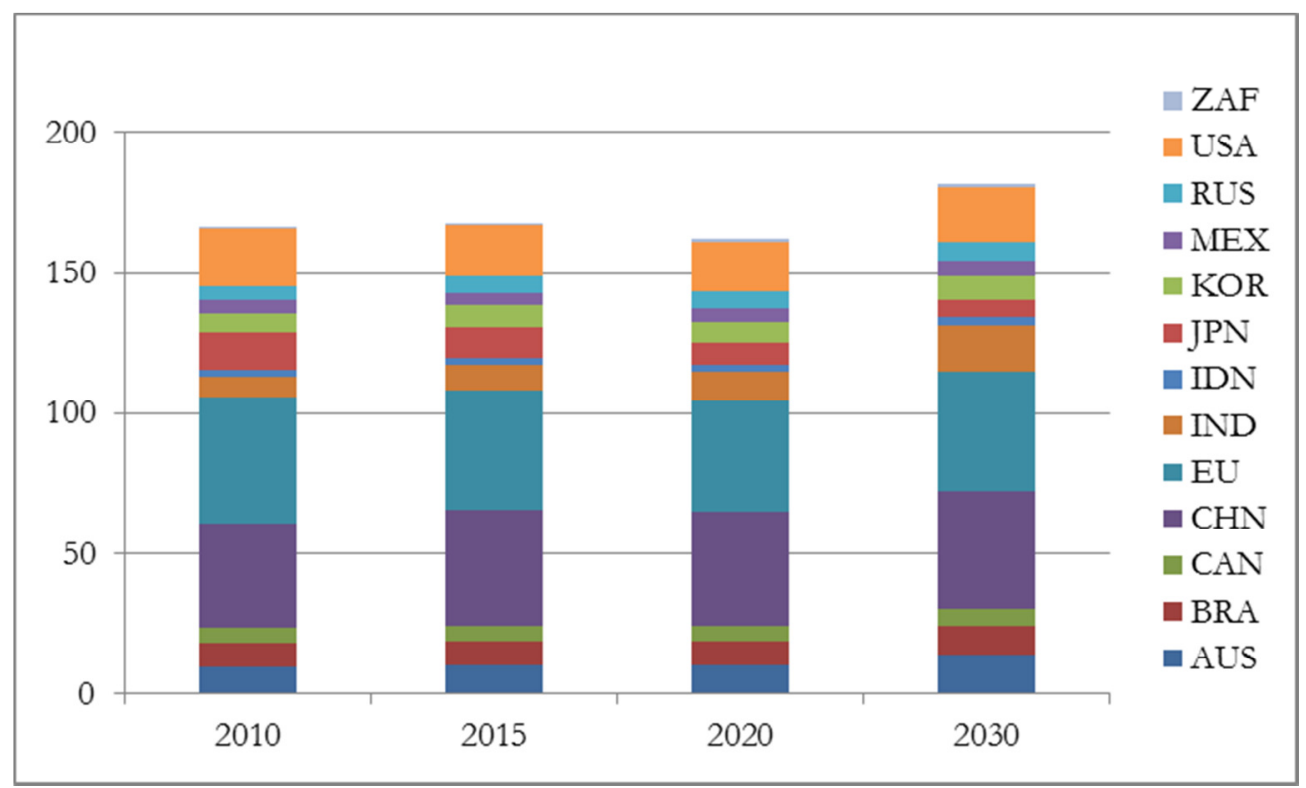

Figure 5-4. Energy Consumption of TVs in Selected Countries

\subsection{Energy Savings Potential}

We evaluated energy savings potential at three points in time; after three years of a market transformation program, i.e., in 2015, then in 2020 and 2030. Because of the rapidly evolving nature of the technology, the 2020 and 2030 figures must be understood as indicative. Table 5-5 and Figure 5-5 show the results of the energy savings potential analysis. 


\begin{tabular}{|c|c|c|c|c|c|c|}
\hline & \multicolumn{3}{|c|}{ Annual Electricity Savings } & \multicolumn{3}{|c|}{ Cumulative Electricity Savings from 2012} \\
\hline & in 2015 & in 2020 & in 2030 & through 2015 & through 2020 & through 2030 \\
\hline & TWh & TWh & TWh & TWh & TWh & TWh \\
\hline Australia & 0.2 & 0.5 & 1.0 & 0.3 & 2.2 & 10.5 \\
\hline Brazil & 0.1 & 0.5 & 0.8 & 0.3 & 2.0 & 9.1 \\
\hline Canada & 0.1 & 0.3 & 0.4 & 0.1 & 1.1 & 4.6 \\
\hline China & 0.8 & 2.1 & 3.3 & 1.7 & 9.5 & 39.0 \\
\hline $\mathrm{EU}$ & 0.6 & 1.8 & 3.2 & 1.3 & 8.0 & 35.0 \\
\hline India & 0.1 & 0.5 & 1.3 & 0.2 & 2.1 & 11.8 \\
\hline Indonesia & 0.1 & 0.1 & 0.2 & 0.1 & 0.4 & 2.3 \\
\hline Japan & 0.1 & 0.2 & 0.4 & 0.2 & 1.1 & 4.9 \\
\hline Korea & 0.2 & 0.4 & 0.6 & 0.4 & 1.8 & 7.2 \\
\hline Mexico & 0.1 & 0.2 & 0.4 & 0.1 & 0.8 & 4.3 \\
\hline Russia & 0.1 & 0.3 & 0.5 & 0.2 & 1.2 & 5.5 \\
\hline South Africa & 0.0 & 0.0 & 0.1 & 0.0 & 0.1 & 0.9 \\
\hline U.S. & 0.7 & 1.3 & 1.4 & 1.5 & 7.3 & 20.9 \\
\hline Total & 3.2 & 8.2 & 13.6 & 6.4 & 37.6 & 156.0 \\
\hline
\end{tabular}

Table 5-5. Annual/Cumulative Electricity Savings Potential for 2015, 2020, and 2030

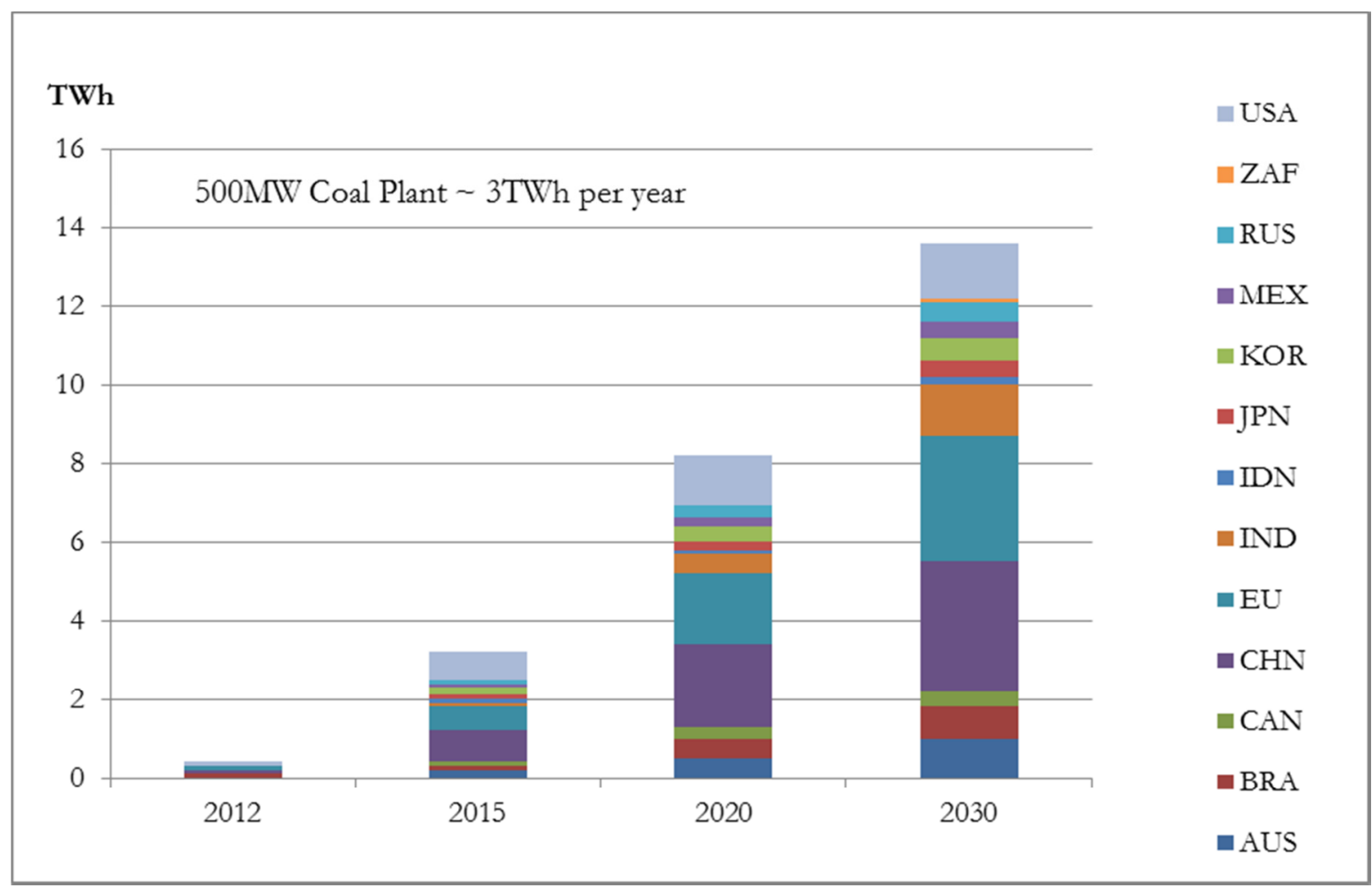

Figure 5-5. Annual Electricity Savings Potential from 2012 to 2030 


\subsection{Emission Savings Potential}

We calculated emission savings potentials from the energy savings potential results above. Emission factors and growth rates for the selected countries were taken from Price et al. (2006) and IEA (2006). As above, because of the rapidly evolving nature of the technology, the 2020 and 2030 figures must be understood as indicative. Our calculation shows that about 1.4 million tons of $\mathrm{CO}_{2}\left(\mathrm{Mt} \mathrm{CO}_{2}\right)$ can be saved annually in 2015 and about $6 \mathrm{Mt} \mathrm{CO}_{2}$ in 2030 thanks to energy savings from the efficiency scenario. Table 5-6 and Figure 5-6 show the results of the emission savings potential analysis.

\begin{tabular}{|c|c|c|c|c|c|c|}
\hline & \multicolumn{3}{|c|}{ Annual Emission Savings } & \multicolumn{3}{|c|}{ Cumulative Emission Savings from 2012} \\
\hline & in 2015 & in 2020 & in 2030 & through 2015 & in 2020 & in 2030 \\
\hline & Mt & Mt & Mt & Mt & Mt & $\mathrm{Mt}$ \\
\hline Australia & 0.26 & 0.75 & 1.18 & 0.58 & 3.37 & 13.86 \\
\hline Brazil & 0.13 & 0.42 & 0.79 & 0.29 & 1.83 & 8.37 \\
\hline Canada & 0.01 & 0.04 & 0.07 & 0.03 & 0.17 & 0.78 \\
\hline China & 0.02 & 0.05 & 0.08 & 0.04 & 0.22 & 0.96 \\
\hline EU & 0.23 & 0.66 & 1.05 & 0.51 & 2.96 & 12.26 \\
\hline India & 0.13 & 0.48 & 1.13 & 0.26 & 1.95 & 10.48 \\
\hline Indonesia & 0.03 & 0.09 & 0.17 & 0.06 & 0.37 & 1.74 \\
\hline Japan & 0.04 & 0.11 & 0.16 & 0.09 & 0.51 & 1.99 \\
\hline Korea & 0.05 & 0.15 & 0.26 & 0.11 & 0.68 & 2.92 \\
\hline Mexico & 0.05 & 0.16 & 0.27 & 0.11 & 0.70 & 3.04 \\
\hline Russia & 0.03 & 0.09 & 0.14 & 0.07 & 0.40 & 1.63 \\
\hline South Africa & 0.01 & 0.04 & 0.06 & 0.03 & 0.17 & 0.71 \\
\hline U.S. & 0.42 & 0.75 & 0.81 & 0.95 & 4.33 & 12.14 \\
\hline Total & 1.41 & 3.79 & 6.17 & 3.12 & 17.66 & 70.87 \\
\hline
\end{tabular}

Table 5-6. Annual Emission Savings Potential for 2015, 2020, and 2030

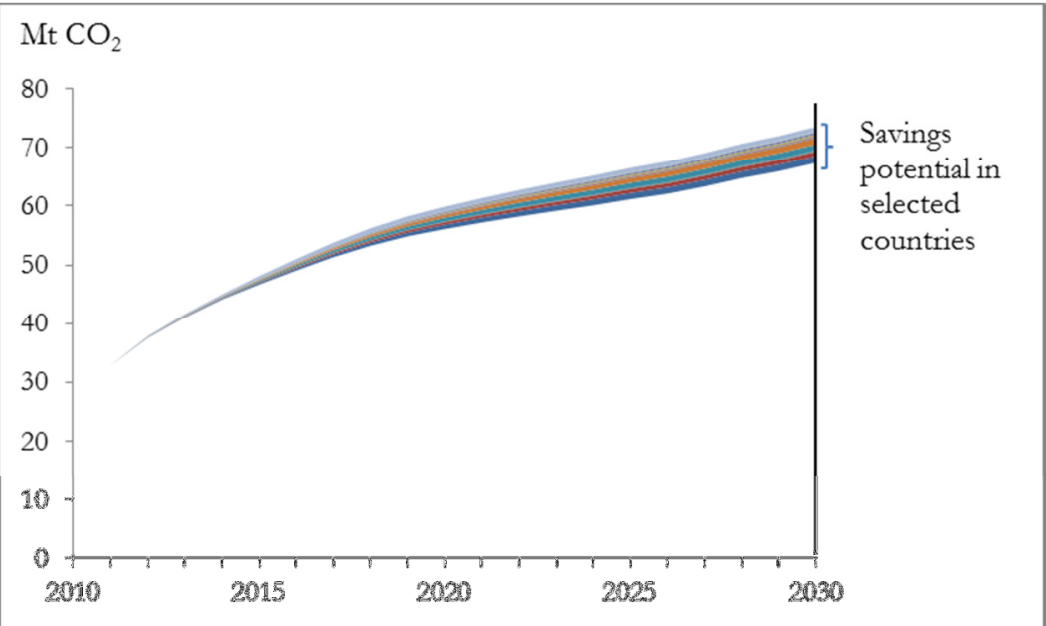

Figure 5-6. Emission Savings Potential from 2012 to 2030 


\section{Other Issues Related to Power Consumption and Efficiency}

The subsections below discuss issues related to TV efficiency that are not discussed or fully addressed in earlier sections of this report: on-mode and rated power, brightness and power consumption, ABC, the learning effect, and standby power.

\subsection{On-Mode Power and Rated Power}

TV efficiency standards focus on on-mode power, which is typically measured using the method specified by IEC 62087 Section 11. However, the on-mode power is not necessarily the same as the rated power of a TV. As mentioned earlier, different physical principles govern energy consumption for each type of screen technology. Power consumption of self-emissive displays such as PDPs and OLEDs varies with the signal images. Consumption of conventional LCD and projection displays is independent of the input signal. LED backlit LCD TVs can control backlight lamps according to the image signal to varying degrees depending on the type of dimming technology.

The ENERGY STAR list does not include rated power data, but the ECCJ website lists rated power data for efficient TV products available in Japan. ${ }^{69}$ Although the ECCJ data do not include on-mode power consumption, it can be back calculated from annual energy consumption, standby power, and assumed viewing hours. ${ }^{70}$ Although the IEC 62087 standard video clip does not measure on-mode power for Japanese products, it is useful to analyze the ECCJ data to compare rated power and on-mode power. Figure 6-1 shows that the on-mode power consumption trend of ENERGY STAR Version 4-qualified TVs.

${ }^{69}$ http://www.eccj.or.jp/cgi-bin/real-catalog/index.php

70 On-mode power is calculated from the following annual energy consumption formula:

http://www.eccj.or.jp/law06/machine/tv_220218.pdf $E_{\text {annual }}(\mathrm{kWh} / \mathrm{yr})=\left\{P_{\text {on mode }} \times\left(\frac{4.5 \mathrm{hrs}}{\text { day }}\right)+P_{\text {standby }} \times\left(\frac{19.5 \mathrm{hrs}}{\text { day }}\right)\right\} \times 365$ 


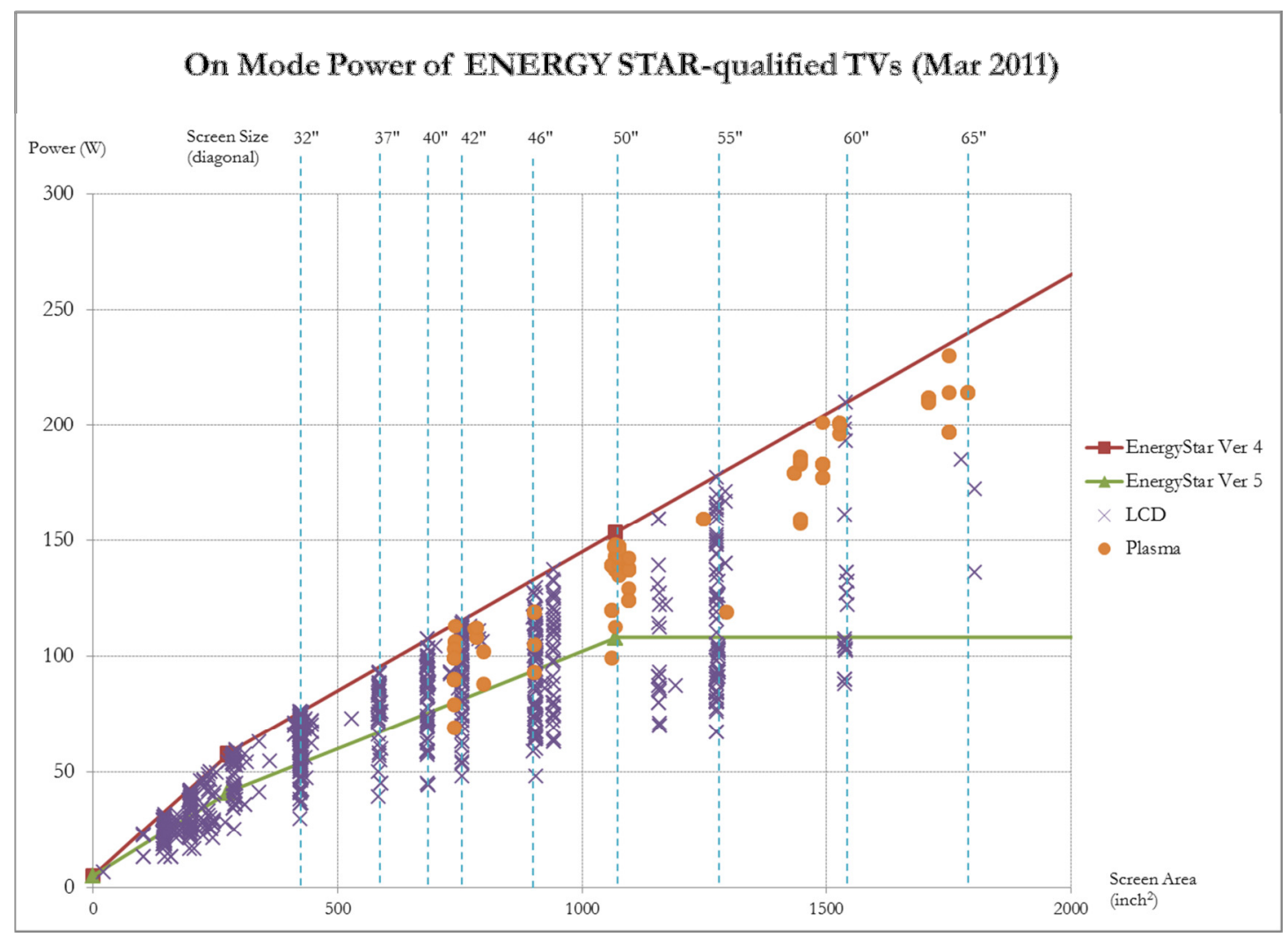

Figure 6-1. On-Mode Power of TVs listed in ENERGY STAR

About 36\% of the total ENERGY STAR Version 4-qualified TVs on the list meet ENERGY STAR Version 5 criteria. In particular, five Panasonic PDP TV models are included among the ENERGY STAR Version 5qualified TVs. The ECCJ TV data (Figures 6-2 and 6-3) show an on-mode power trend that is consistent with the ENERGY STAR data shown in Figure 6-1, but the ECCJ rated-power and on-mode data are not consistent. The gap between rated-power and on-mode power increases with increasing screen size for the ECCJ data. 


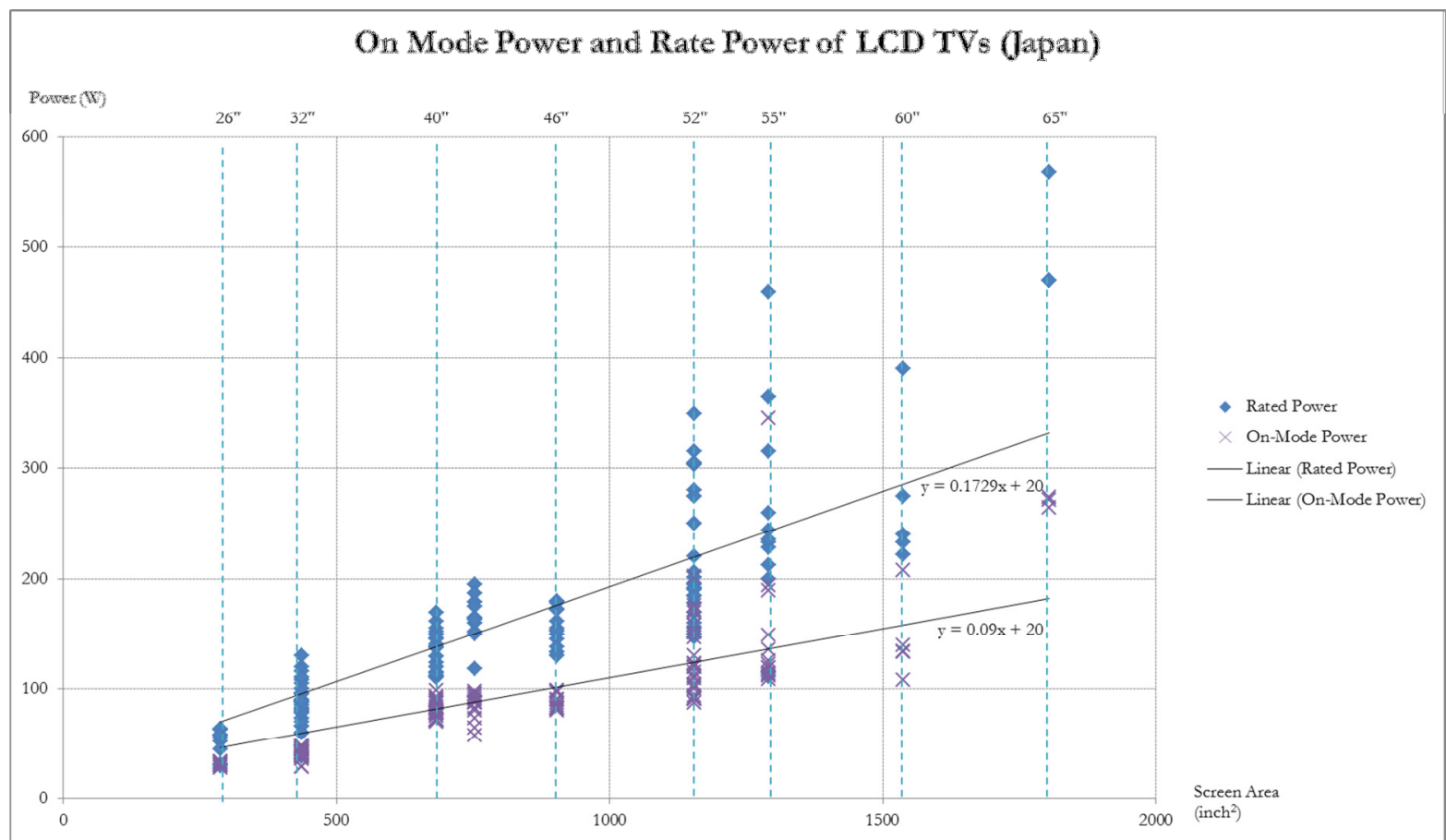

Figure 6-2. On-Mode Power and Rated Power of LCD TVs (Japan)

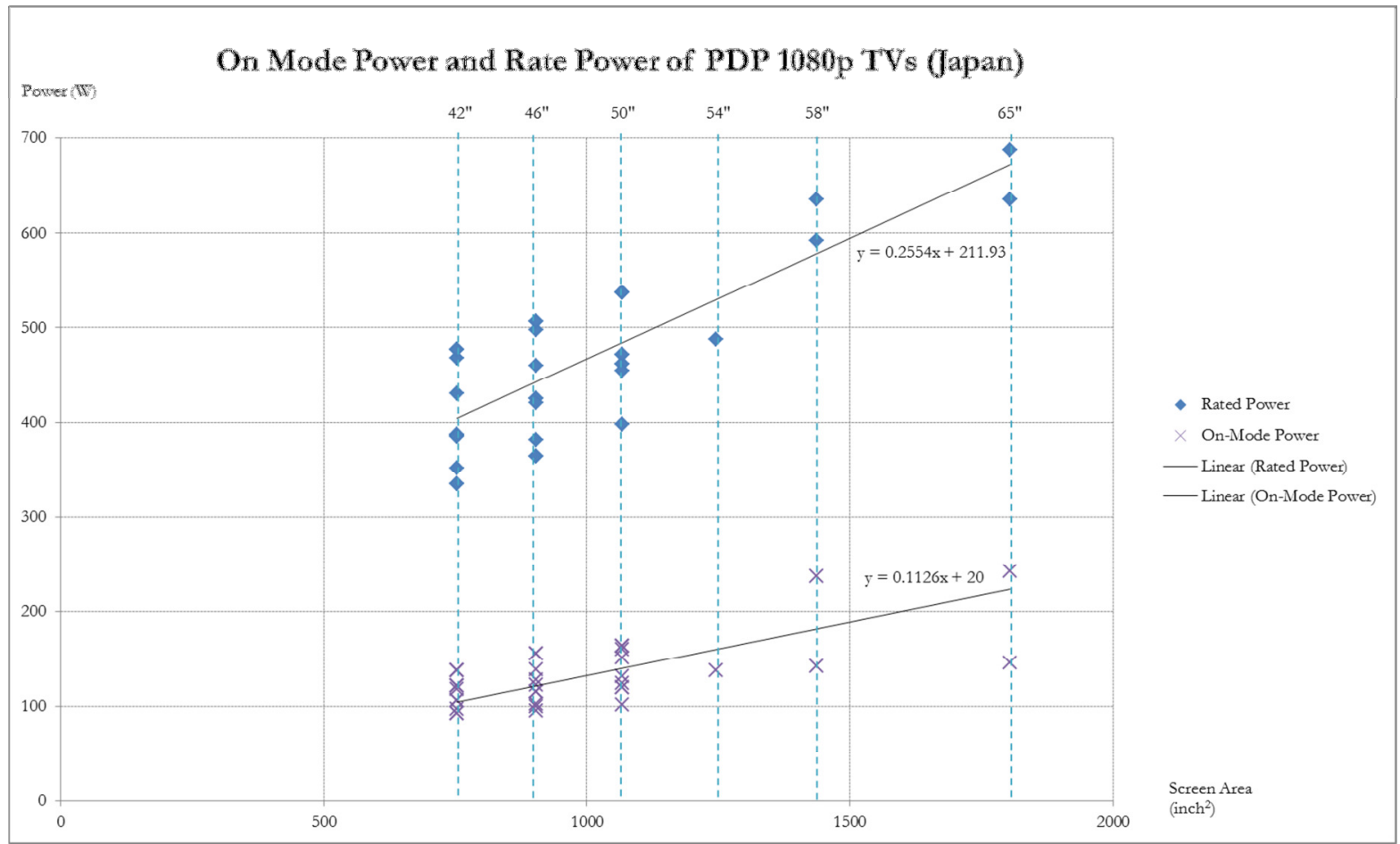

Figure 6-3. On-Mode Power and Rated Power of PDP TVs (Japan)

Within each screen size class, the focus appears to have been on minimizing on-mode power levels regardless of rated power levels. For example, the rated power of 32-inch LCD TVs is between $38 \mathrm{~W}$ and $130 \mathrm{~W}$, but the on-mode power for most of the same TVs is close to $40 \mathrm{~W}$. In contrast, PDP TV on-mode power is much lower than rated power. For example, the rated power of 42-inch PDP TVs is between $335 \mathrm{~W}$ and 477 $\mathrm{W}$, but on-mode power of the same TVs is between $93 \mathrm{~W}$ and $138 \mathrm{~W}$, which is $28 \%$ to $32 \%$ of the rated 
power levels. Power consumption of PDPs, which are self-emissive displays, varies with the TV signal and can be also controlled by ABC. Figure 6-4 shows ECCJ data for on-mode and rated power of 32-inch LCD and 42-inch PDP TVs.
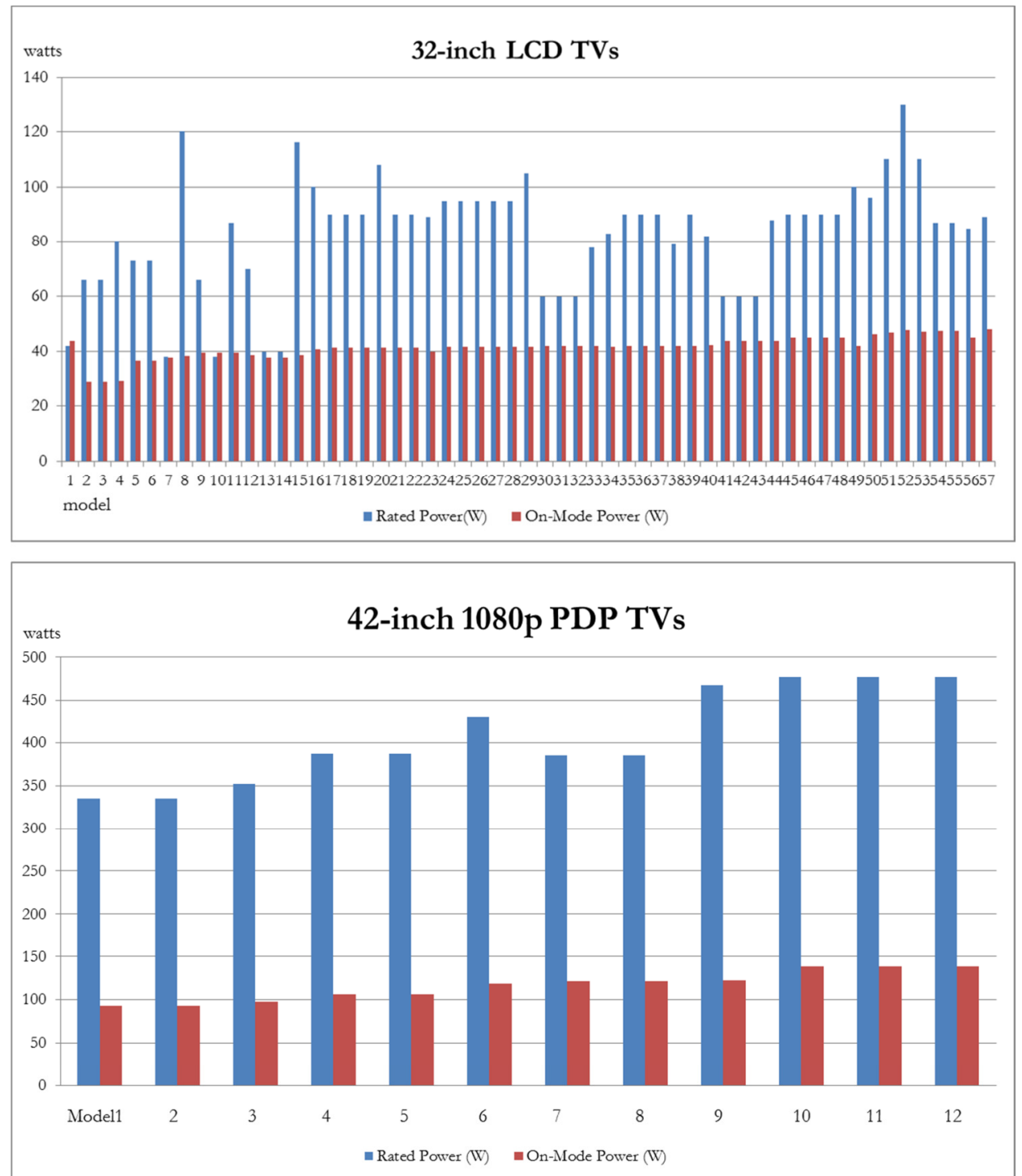

Figure 6-4. On-Mode and Rated Power of 32-inch LCD and 42-inch PDP TVs (Japan) 


\subsection{Brightness and Power Consumption}

Brightness control functions - e.g., $\mathrm{ABC}$ for external light conditions, dimming techniques in LCD backlights, auto power control in PDPs, and auto current limit in OLEDs - play a significant role in controlling a TV's on-mode power. However, currently available TVs still consume more power in modes other than the default home mode. According to Ecos (Ecos 2010), the effect of various display settings on TV power consumption was significant for many models which were manufactured between 2007 and 2009. According to the observation, "the effect of display settings on TV power consumption is profound - for many models, differences of 2:1 or even 3:1 were observed from the lowest to highest power - consuming combination of settings."(Ecos 2010) That is mainly because luminance values vary across available presets on each TV model. This means that current TVs have potentials to greatly increase or decrease power consumption, depending on display settings. In fact, according to ENERGY STAR 2011, luminance in default mode for LCD and PDP TVs varies from 53 to $527 \mathrm{~cd} / \mathrm{m}^{2}$. As this wide range may suggest that some consumers would adjust their TV's display settings from default home mode to other modes for their preferences, resulting in power increase or decrease. Figure 6-5 illustrates an example of power consumption for a 46-inch LED-LCD TV in various modes.

\section{TV F LCD-LED 46"}

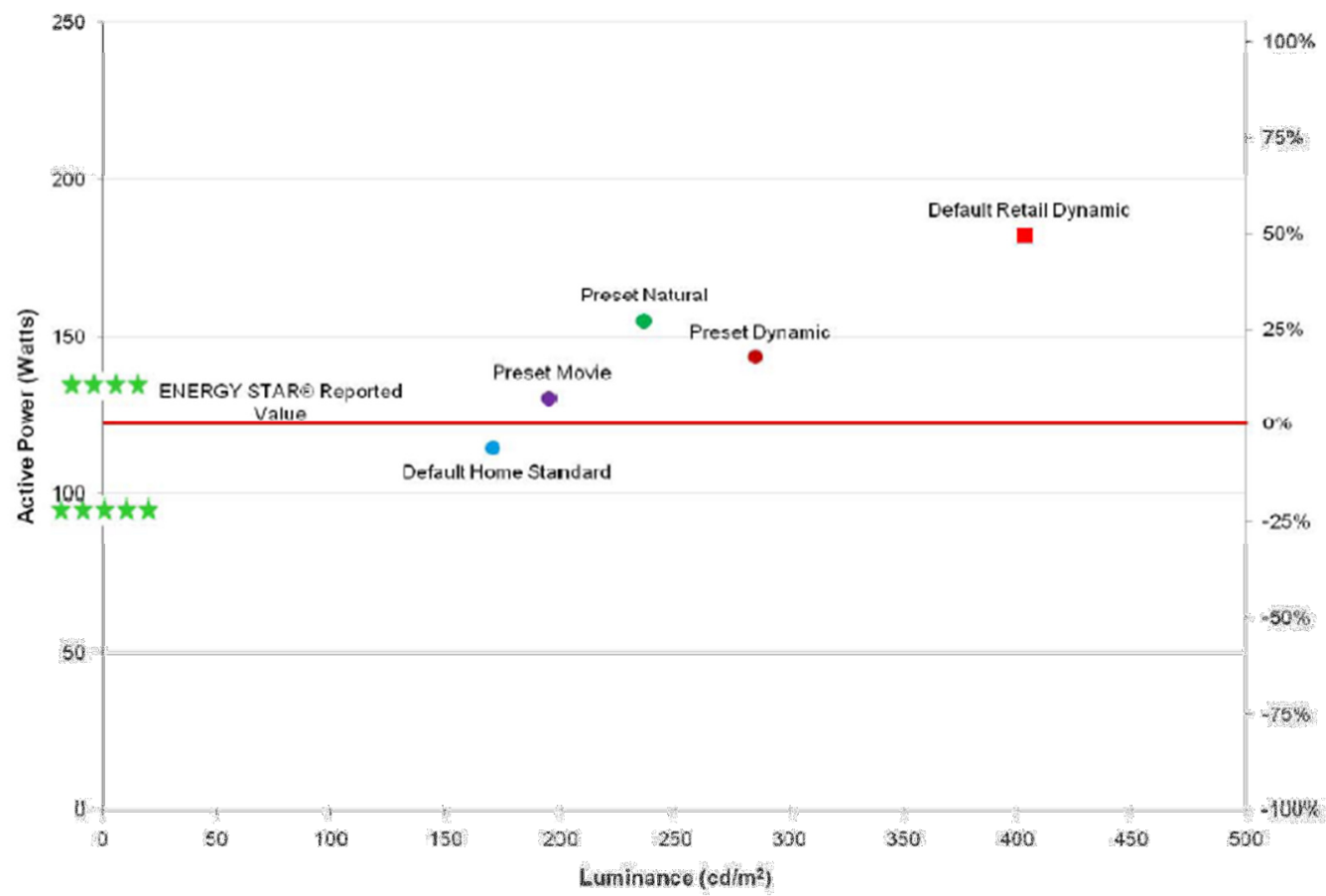

Source: Ecos 2010

Figure 6-5. Power Consumption of a 46-inch LED-LCD TV in Various Modes

As mentioned earlier, LCD TV panels larger than 30 inches have been manufactured with a target luminance of 400 to $500 \mathrm{~cd} / \mathrm{m}^{2}$. In general, the luminance corresponds to retail mode or to the brightest selectable mode whereas the luminance in default mode, in which TVs are shipped, is lower than the maximum level. The ENERGY STAR standard states: "To qualify as ENERGY STAR under the specification, the peak luminance of the product in the 'bome' mode, or in the default mode as shipped, shall not be less than 65\% of the peak luminance of the 'retail' mode, or the brightest selectable preset mode of the product." In fact, a 32-inch LCD TV's average luminance in default mode was about $75 \%$ of the average luminance in the brightest selectable preset mode. The most efficient models in this class all have similar features, including ABC enabled as shipped. Some models from major brands show relatively low luminance levels in both modes. Even though overall TV efficiency has been getting better, brightness control plays an important part in low TV power consumption. Table 6-1 
shows the luminance of 32-inch LCD ENERGY STAR TVs in default and brightest modes.

\begin{tabular}{|c|c|c|c|c|c|c|c|}
\hline Brand & Model & Resolution & $\mathrm{ABC}$ & $\begin{array}{l}\text { On- } \\
\text { Mode } \\
\text { Power } \\
\text { (W) }\end{array}$ & $\begin{array}{l}\text { Luminance in } \\
\text { Default Mode as } \\
\text { Shipped }(\mathrm{cd} / \mathrm{m} 2) \\
\text { (a) }\end{array}$ & $\begin{array}{l}\text { Luminance in } \\
\text { Brightest } \\
\text { Selectable Preset } \\
\text { Mode }(\mathrm{cd} / \mathrm{m} 2) \\
\text { (b) }\end{array}$ & (a)/(b) \\
\hline Samsung & $\begin{array}{l}\text { UN32D4000ND } \\
\text { UN32D5500RF } \\
\text { LN32D550K1F }\end{array}$ & $\begin{array}{l}1366 \times 768 \\
1920 \times 1080 \\
1366 \times 768 \\
\end{array}$ & $\begin{array}{l}\mathrm{N} \\
\mathrm{Y} \\
\mathrm{Y}\end{array}$ & $\begin{array}{l}30 \\
37 \\
42 \\
\end{array}$ & $\begin{array}{l}199 \\
330 \\
278 \\
\end{array}$ & $\begin{array}{l}210 \\
343 \\
322 \\
\end{array}$ & $\begin{array}{l}0.95 \\
0.96 \\
0.86\end{array}$ \\
\hline VIZIO & $\begin{array}{l}\text { E320VP } \\
\text { M320NV }\end{array}$ & $\begin{array}{l}1366 \times 768 \\
1920 \times 1080\end{array}$ & $\begin{array}{l}\mathrm{Y} \\
\mathrm{Y}\end{array}$ & $\begin{array}{l}36 \\
52\end{array}$ & $\begin{array}{l}361 \\
305\end{array}$ & $\begin{array}{l}433 \\
396\end{array}$ & $\begin{array}{l}0.83 \\
0.77\end{array}$ \\
\hline LG & 32LV25 & $1366 \times 768$ & $\mathrm{Y}$ & 41 & 250 & 331 & 0.76 \\
\hline HAIER & LE32C1320 & $1366 \times 768$ & $\mathrm{~N}$ & 41 & 260 & 331 & 0.79 \\
\hline Sony & $\begin{array}{l}\text { KDL-32EX520 } \\
\text { KDL-32EX720 }\end{array}$ & $\begin{array}{l}1920 \times 1080 \\
1920 \times 1080\end{array}$ & $\begin{array}{l}\mathrm{Y} \\
\mathrm{Y}\end{array}$ & $\begin{array}{l}47 \\
48\end{array}$ & $\begin{array}{l}192 \\
219\end{array}$ & $\begin{array}{l}283 \\
306\end{array}$ & $\begin{array}{l}0.68 \\
0.72\end{array}$ \\
\hline Panasonic & $\begin{array}{l}\text { TC-L32E3 } \\
\text { TC-32LX34 } \\
\text { TC-L32C3 } \\
\end{array}$ & $\begin{array}{l}1920 \times 1080 \\
1366 \times 768\end{array}$ & $\begin{array}{l}\mathrm{Y} \\
\mathrm{Y} \\
\mathrm{Y} \\
\end{array}$ & $\begin{array}{l}38 \\
42 \\
42 \\
\end{array}$ & $\begin{array}{l}302 \\
218 \\
228 \\
\end{array}$ & $\begin{array}{l}346 \\
302 \\
327 \\
\end{array}$ & $\begin{array}{l}0.88 \\
0.72 \\
0.70 \\
\end{array}$ \\
\hline \multicolumn{3}{|c|}{ Average of total 32-inch LCD TVs $(n=167)$} & $\begin{array}{l}\mathrm{Y}(81) \\
\mathrm{N}(86)\end{array}$ & 63 & 269 & 357 & 0.75 \\
\hline
\end{tabular}

Source: ENERGY STAR 2011

\section{Table 6-1. Luminance of 32-inch ENERGY STAR TVs in Default and Brightest Modes}

\subsubsection{Auto Brightness Control (ABC)}

ENERGY STAR Version 5 criteria (ENERGY STAR 2011b) specify the following equation to determine onmode power of a TV in which $A B C$ is activated by default when the TV is shipped to the end user:

$P_{a} 1_{\text {broadcast }}=\left(0.55 \times P_{\text {o-broadcast }}\right)+\left(0.45 \times P_{\text {abc-broadcast }}\right)$

Where:

$\mathbf{P}_{\mathbf{a}}$ 1_broadcast: "the average on-mode power consumption in watts and rounded to the nearest whole number, taking into consideration that the TV will be in low ambient light level conditions $45 \%$ of the time."

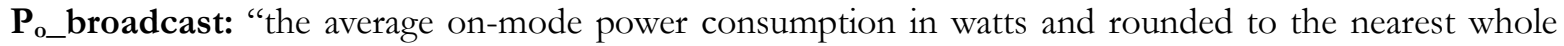
number, and tested with a minimum ambient light level of 300 lux entering into the sensor."

$\mathbf{P}_{\text {abc: }}$ "the average on-mode power consumption in watts and rounded to the nearest whole number, with an ambient light level of zero (0) lux measured at the face of the sensor."

On-mode power of TVs in which ABC is activated by default is expected to be lower than for other TVs. To compare the effect of $\mathrm{ABC}$, we categorized the TVs in the ENERGY STAR list according to their ABC status as shown in Table 6-2.

\begin{tabular}{l|c|c|c}
\hline & $\begin{array}{c}\text { Total Number of } \\
\text { TVs }\end{array}$ & $\begin{array}{c}\text { Number of TVs with } \\
\text { ABC Enabled when } \\
\text { Shipped }\end{array}$ & $\begin{array}{c}\text { Number of TVs with No ABC or ABC } \\
\text { Not Enabled when Shipped }\end{array}$ \\
\hline LCD TV & 920 & 532 & 388 \\
\hline PDP TV & 119 & 116 & 3 \\
\hline
\end{tabular}

Table 6-2. ABC Status of ENERGY STAR TVs (Mar 22, 2011)

Table 6-2 shows that more than 50\% of ENERGY STAR Version 4-qualified LCD TVs have ABC enabled when they are shipped. In addition, almost all PDP TVs that qualify for ENERGY STAR Version 4 have 
$\mathrm{ABC}$ enabled when shipped, and virtually all TVs in the current market with screens larger than 30 inches that meet ENERGY STAR Version 5 criteria have ABC enabled when shipped. Figures 6-6 and 6-7 show onmode power of TVs with and without $\mathrm{ABC}$ default settings. $\mathrm{ABC}$ appears to be essential for PDP TVs to meet ENERGY STAR criteria. It is expected that $A B C$ will continue to play a significant role in minimizing TV power consumption.

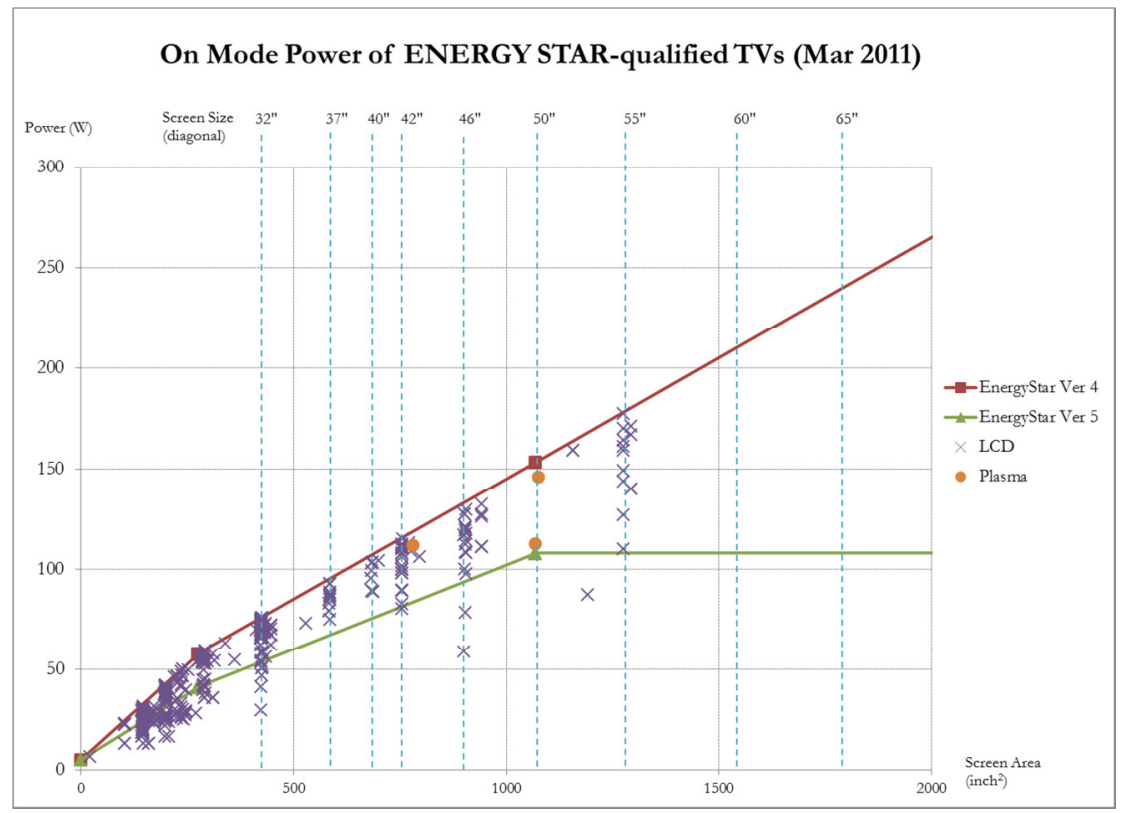

${ }^{*} \mathrm{~N}_{\text {abc: }}$ TVs that do not have $\mathrm{ABC}$ or whose $\mathrm{ABC}$ is NOT enabled by default when the TVs are shipped.

Figure 6-6. On-mode Power of TVs without ABC Default Setting $\left(\mathbf{N}_{\text {abc }}\right)$

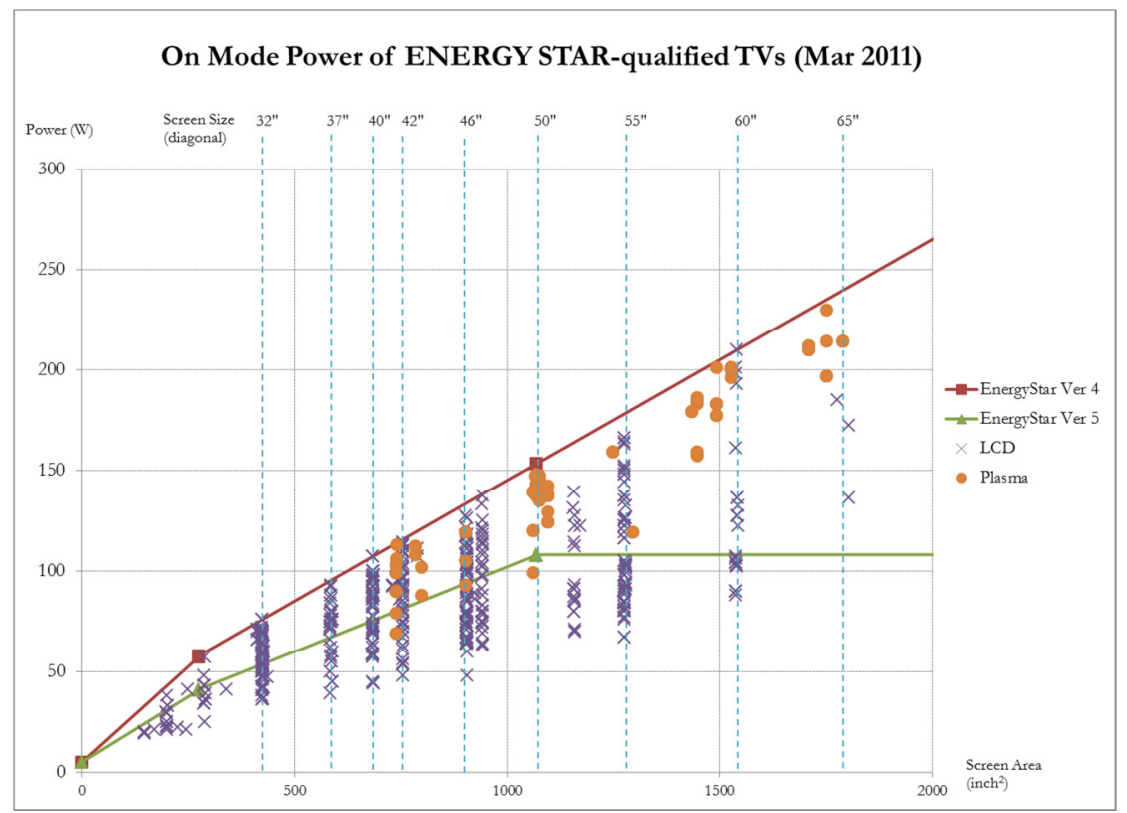

* $Y_{\text {abc }}$ TVs whose ABC function is enabled by default when the TVs are shipped.

Figure 6-7. On-mode Power of TVs with ABC Default Setting $\left(\mathrm{Y}_{\mathrm{abc}}\right)$

While $\mathrm{ABC}$ is a function that can easily save TV's total energy consumption, the ENERGY STAR Version 5 test method is flawed because it tests TVs at 0 and 300 lux. According to experts, the test method needs to be improved because zero lux is too low, and some manufacturers are dimming the screens to unacceptably dark 
level to obtain a low power reading. The Swedish Energy Agency also commented that it is important to provide manufacturers with guidance for $A B C$ settings and that the test procedure needs to prevent the $A B C$ function from being used counterproductively to bypass efficiency requirements such as ENERGYSTAR Version 5. These comments refer to $\mathrm{ABC}$ settings that allow manufacturers to claim energy savings during testing, but the TV purchaser is then prompted to disable the ABC for a brighter screen, which would mean that the energy savings documented by the test procedure would not be achieved when the TV is actually operated by a user. All major manufacturers provide at least one model of TV that is shipped with ABC activated. To ensure that $\mathrm{ABC}$ is properly used as an efficiency improvement option:

- $\mathrm{ABC}$ needs to be activated by default for all TVs eligible to participate in market transformation programs.

- $\mathrm{ABC}$ needs to be easier to adjust than to deactivate completely.

- The ENERGY STAR test method to account for ABC needs to be revised to test at more realistic ambient lighting levels.

The draft ENERGY STAR Version 6 requirements (ENERGY STAR 2011c) include a revised method for ABC. On-mode power consumption for TVs with ABC is expected to be calculated as a weighted average of power consumption at various ambient lighting levels that are agreed upon by manufacturers.

\section{Effect of ABC on On-mode Power of ENERGY STAR-qualified TVs}

Figure 6-8 shows distribution of ENERGY STAR-qualified LCD TVs by ABC setting. ABC is typically enabled when large screen size $(>40 ")$ TVs are shipped, but there is still significant potential for small and medium screen size $(<40 ")$ TVs to be shipped with $A B C$ enabled, thereby reducing on-mode power consumption. Figure 6-8 shows distribution of ENERGY STAR-qualified LCD TVs by ABC setting.

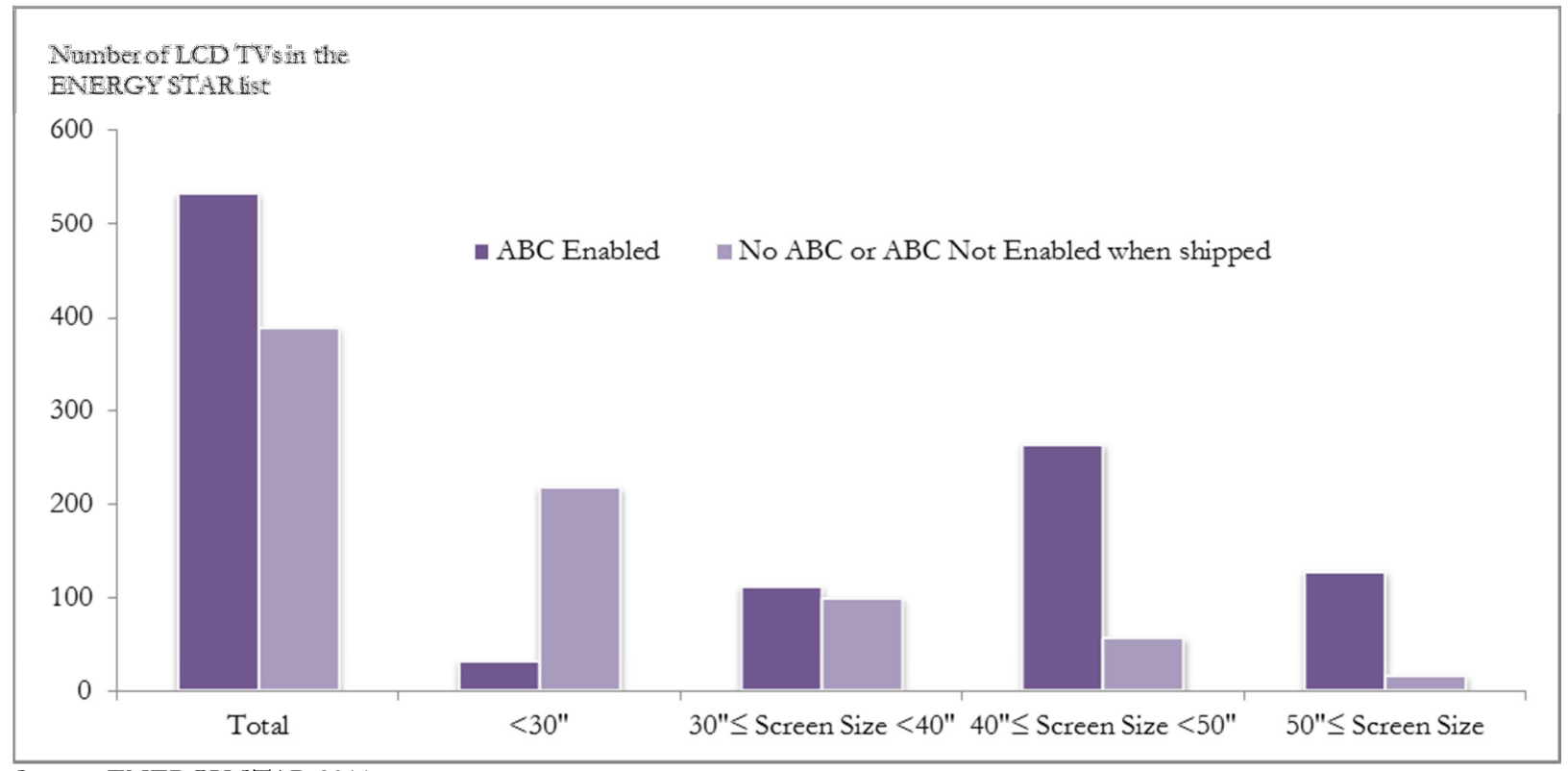

Source: ENERGY STAR 2011

Figure 6-8. Distribution of ENERGY STAR-qualified LCD TVs by ABC Setting

As the ENERGY STAR data set provides only one on-mode power figure for each model, it is difficult to analyze the effect of $\mathrm{ABC}$ on the change in a TV's on-mode power. According to a data set from ICF International, which has provided technical and analytical support for the ENERGY STAR program, 17\% of ENERGY STAR-qualified TV models (only LCD and PDP TVs) do not meet ENERGY STAR Version 4 
criteria with $A B C$ disabled. In particular, it seems that $A B C$ is important for CCFL-LCD TVs and PDP TVs to meet ENERGY STAR criteria. Figure 6-9 shows on-mode power of ENERGY STAR-qualified TVs with ABC disabled.

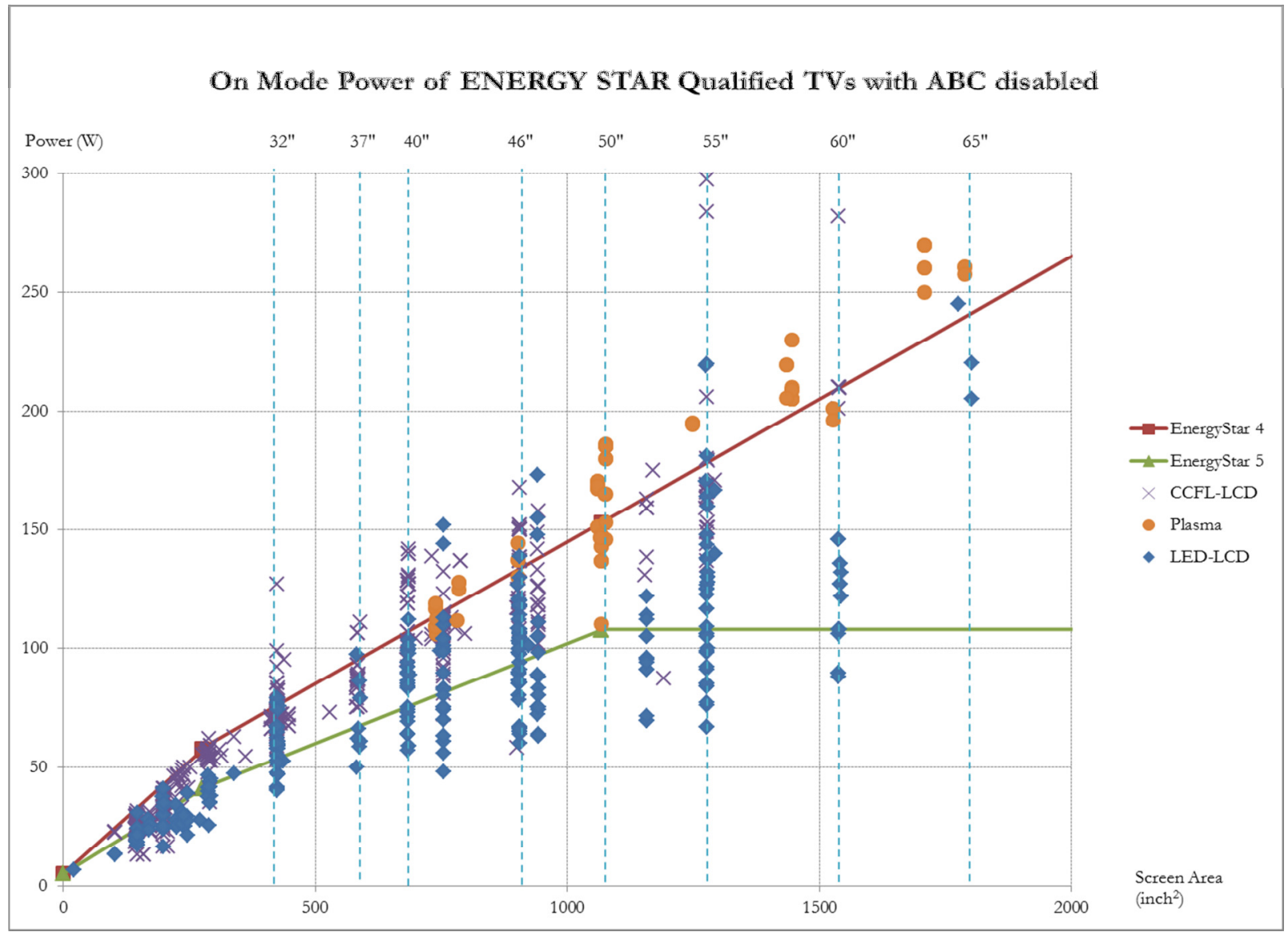

Source: Authors' calculation based on a data set from ICF International (ICF International 2011)

Figure 6-9. On-mode Power of ENERGY STAR-qualified TVs with ABC Disabled

We compared on-mode power data from the ENERGY STAR data set ("ENERGY STAR" below) with onmode power data for the same TV models in default mode with ABC disabled ("ABC-disabled" below). The data were provided by ICF International. Figure 6-10 shows the results for main screen size categories. Based on the findings, the average on-mode power of ENERGY STAR TVs with ABC enabled is $8 \%$ to $20 \%$ less than the average in case $\mathrm{ABC}$ settings are disabled, depending on screen or backlight technology and screen size. 


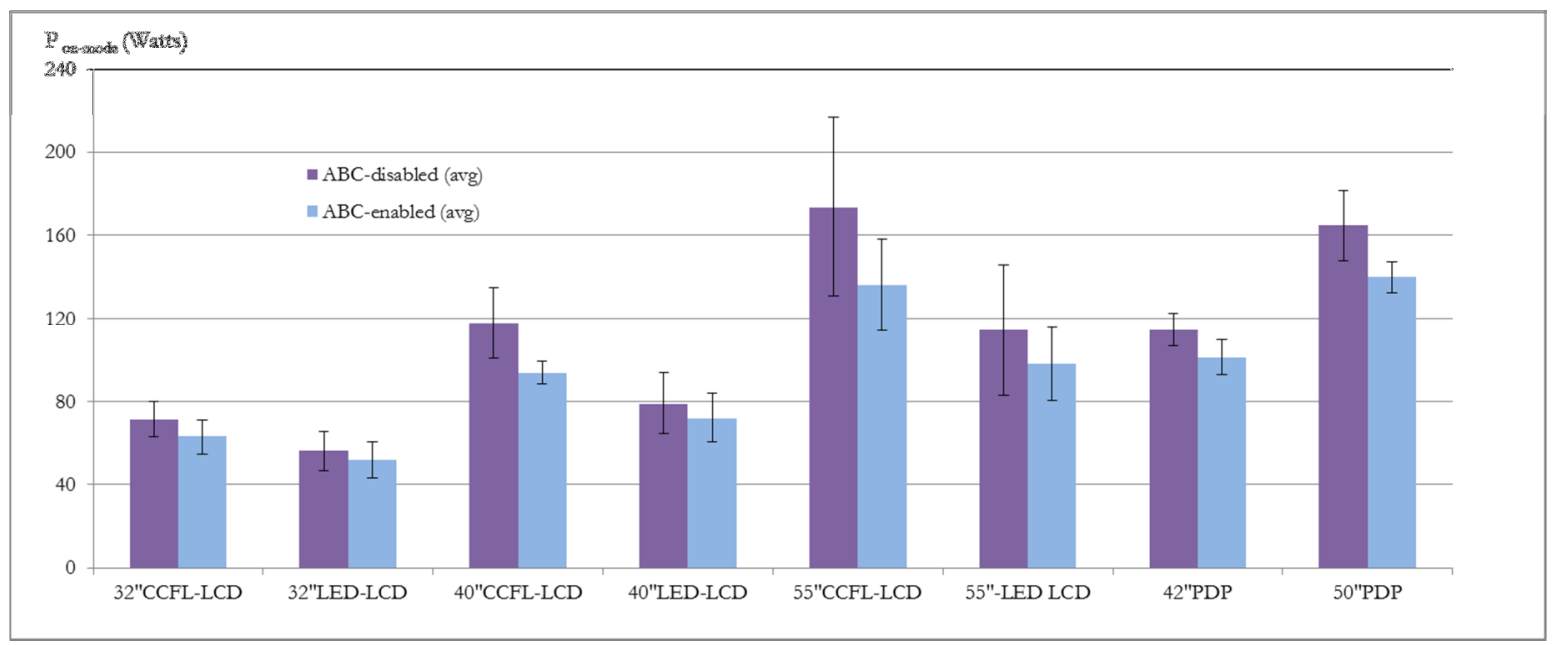

Source: Authors' calculation based on ICF International 2011 and ENERGY STAR 2011

Figure 6-10. Effect of ABC Setting on Average On-mode Power of ENERGY STAR TVs

\section{a. 32-inch LCD T'Vs}

The average on-mode power of 44 ENERGY STAR-qualified 32-inch CCFL-LCD TV models with ABC enabled is $63 \mathrm{~W}$. The average on-mode power of the same 44 models with ABC disabled is $71 \mathrm{~W}$. Likewise, the average on-mode power of 24 ENERGY STAR 32-inch LED-LCD TV models is $52 \mathrm{~W}$ with ABC settings enabled. The average on-mode power of the same 24 models with ABC disabled is $56 \mathrm{~W}$. For 32-inch LCD TVs that have ABC settings enabled, the on-mode power was about $5 \%$ to $31 \%$ less than on-mode power in their default modes with $A B C$ settings disabled, except in a few models. Figure 6-11 shows on-mode power distribution of 32-inch LCD TVs with $A B C$ and without ABC.

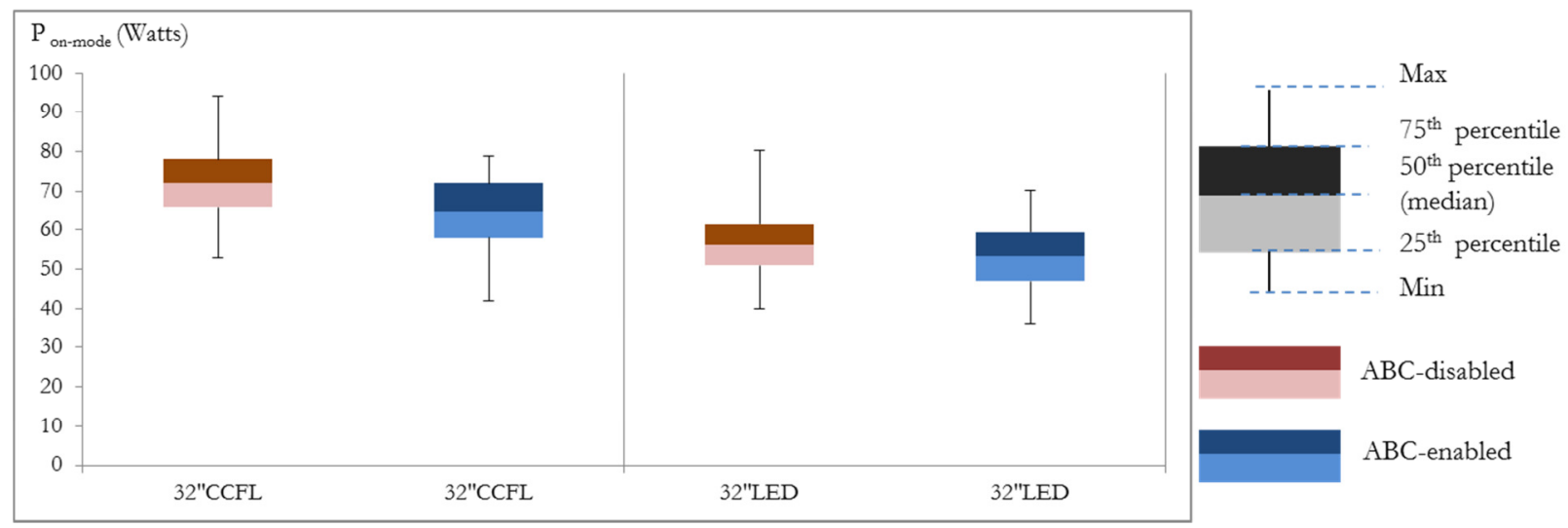

Source: Authors' calculation based on ICF International 2011 and ENERGY STAR 2011

Figure 6-11. On-mode Power of 32-inch LCD TVs with ABC and without ABC

\section{b. 40-inch LCD TVS}

The average on-mode power of 33 ENERGY STAR 40-inch CCFL-LCD TV models with ABC enabled is $94 \mathrm{~W}$. The average on-mode power of the same models with $\mathrm{ABC}$ disabled is $118 \mathrm{~W}$. Likewise, the average on-mode power of 35 ENERGY STAR 32-inch LED-LCD TV models with ABC enabled is $72 \mathrm{~W}$. The average on-mode power of the same models with ABC disabled is $79 \mathrm{~W}$. For 40-inch CCFL-LCD TVs with $\mathrm{ABC}$ enabled, on-mode power was about $7 \%$ to $31 \%$ less than on-mode power in their default modes without ABC. For 40-inch LED-LCD TVs with ABC enabled, on-mode power was about 4\% to $25 \%$ less than on-mode power in their default modes without $\mathrm{ABC}$. Figure 6-12 shows on-mode power distribution of 40-inch LCD TVs with ABC and without ABC. 


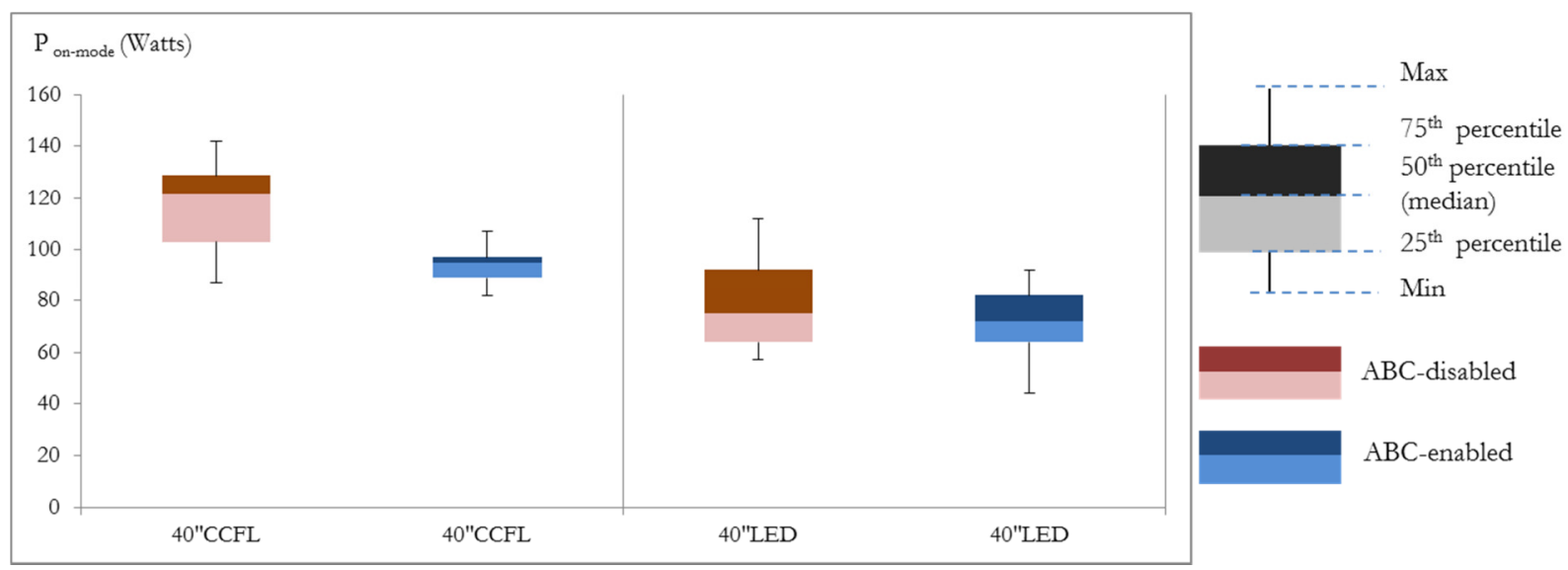

Source: Authors' calculation based on ICF International 2011 and ENERGY STAR 2011

Figure 6-12. On-mode Power of 40-inch LCD TVs

\section{c. PDP TVS}

The average on-mode power of 15 ENERGY STAR 42-inch PDP TVs models with ABC enabled is $101 \mathrm{~W}$. The average on-mode power of the same models with $\mathrm{ABC}$ disabled is $114 \mathrm{~W}$. Likewise, the average on-mode power of 32 ENERGY STAR 50-inch PDP TV models is $140 \mathrm{~W}$. The average on-mode power of the same models with $\mathrm{ABC}$ disabled is $162 \mathrm{~W}$. Figure 6-13 shows on-mode power distribution of PDP TVs with ABC and without $\mathrm{ABC}$.

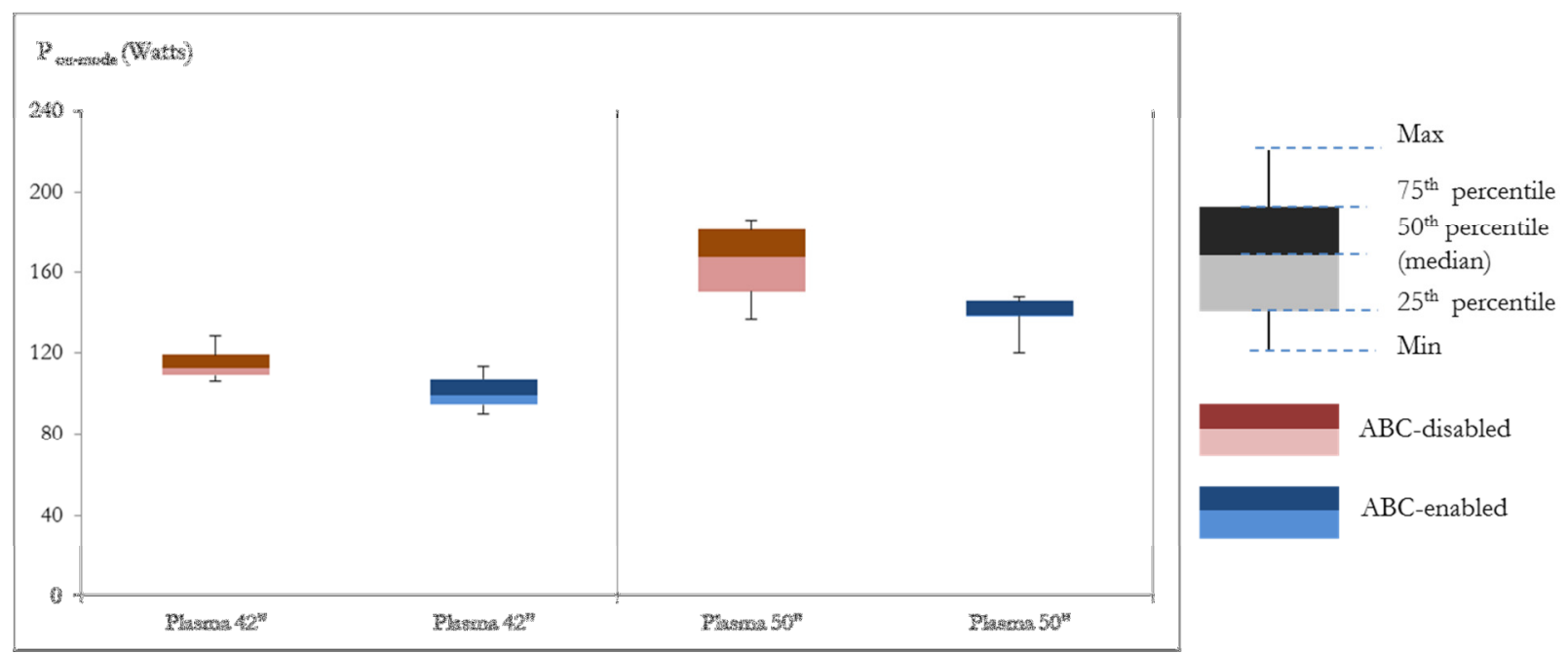

Source: Authors' calculation based on ICF International 2011 and ENERGY STAR 2011

Figure 6-13. On-mode Power of PDP TVs

For 42-inch PDP TVs with ABC enabled, on-mode power was about $12 \%$ to $17 \%$ less than on-mode power in their default modes without ABC. For 50-inch PDP TVs with ABC settings enabled, on-mode power was about $9 \%$ to $23 \%$ less than on-mode power in their default modes without ABC.

The effect of ABC on reduction in on-mode power varies with TV models. According to Ecos (Ecos 2010), implementation of $\mathrm{ABC}$ varies widely with TV models. Some manufacturers implemented $\mathrm{ABC}$ in a linear scale, with screen luminance increasing steadily with increase in ambient light levels. In other cases, luminance of a TV would drop sharply at low ambient light levels, e.g., 0 to 50 lux, but would otherwise be relatively high for all other ambient light conditions. According to one manufacturer, the $\mathrm{ABC}$ was designed to change the screen brightness non- linearly as the ambient light changed linearly. Although some manufacturers may be utilizing the $\mathrm{ABC}$ as means to qualify ENERGY STAR requirements, ABCs are designed to adjust screen 
brightness to a level where consumers feel adequate or comfortable watching TV in given room conditions. Although it is expected that the ABC test method for ENERGY STAR Version 6 will be practically revised, at this stage the effect of $\mathrm{ABC}$ settings on TV on-mode power can be estimated in a range of $10-30 \%$.

\subsection{Technological Learning (Learning by Doing)}

Although we assume that DisplaySearch forecast of future TV prices and costs used in this report include technological learning implicitly, in this section use the experience curve approach to model technological learning for illustrative purposes only.

The experience curve is an empirical model based on historical data fits of price and/or cost (P) data to cumulative production (X). This model has been applied to a wide range of products (U.S. DOE 2011). Although "experience curves" and "learning curves" have identical mathematical forms, they represent different perspectives that are relevant to studies of different scope. According to U.S. DOE (2011), learning curves are generally used when the study focuses on worker and management "learning" that reduces labor hours for a single standardized product from a single manufacturer. Experience curves usually focus on broader classes of products, e.g., all refrigerators, which may encompass many models built by many manufacturers. Because of the range of TV models considered in this study, we discuss experience curves, focusing on LCD and PDP TVs and using global TV shipment data and U.S. TV market prices.

\subsubsection{Mathematical Form}

The experience curve has the following form:

$P(X)=P_{0} X^{-b} \ldots$ (i)

where $P(X)$ represents the price at cumulative production $\mathrm{X}, P_{0}$ stands for the price of the first unit produced, and $\mathrm{b}$ is the learning rate parameter.

The price learning parameter (LR), is represented as follows:

$L R=1-2^{-b} \ldots$ (ii)

and describes the fractional reduction in price expected from each doubling of cumulative production. To obtain the learning rate parameter, Equation (1) is linearized by taking the natural logarithm of both sides of the equation:

$\ln (P(X))=-b \ln (X)+\ln \left(P_{o}\right) \ldots($ iii $)$

\subsubsection{Historical Data for LCD and PDP T'S}

Historical TV shipment and price data are based on DisplaySearch reports (DisplaySearch 2010b, 2010e, $2010 \mathrm{~g}, 2011 \mathrm{a})$. To determine the normalized price of TVs among various screen sizes and models, we calculated volume-weighted average prices for LCD and PDP TVs. Because about 70\% of LCD TVs have screens between 30 and 44 inches, and more than 90\% of PDP TVs have screens between 40 and 50 inches, the volume-weighted price of PDP TVs is higher than that of LCD TVs, even though the average price of PDP TVs is lower than that of LCD TVs in the same screen size. Table 6-3 shows the volume-weighted average prices of LCD TVs and PDP TVs. 


\begin{tabular}{|c|c|c|c|c|}
\hline & \multicolumn{2}{|c|}{ LCD TVs } & \multicolumn{2}{|c|}{ PDP TVs } \\
\hline & Average Price (\$) & $\begin{array}{c}\text { Global Market } \\
\text { Share }(\%)\end{array}$ & Average Price (\$) & $\begin{array}{c}\text { Global Market } \\
\text { Share }(\%)\end{array}$ \\
\hline 15"-19" & $\$ 193$ & $8.0 \%$ & - & - \\
\hline $20 "-21 "$ & $\$ 259$ & $0.9 \%$ & - & - \\
\hline $25 "-29 "$ & $\$ 319$ & $9.9 \%$ & - & - \\
\hline 30"-34" & $\$ 498$ & $40.7 \%$ & - & $0.0 \%$ \\
\hline $35 "-39 "$ & $\$ 592$ & $7.9 \%$ & $\$ 509$ & $1.3 \%$ \\
\hline 40"-44" & $\$ 798$ & $21.2 \%$ & $\$ 638$ & $56.5 \%$ \\
\hline $45 "-49 "$ & $\$ 1,062$ & $8.0 \%$ & $\$ 785$ & $2.8 \%$ \\
\hline 50"-54" & $\$ 1,194$ & $1.2 \%$ & $\$ 1,057$ & $35.7 \%$ \\
\hline $55 "-59 "$ & $\$ 1,766$ & $2.0 \%$ & $\$ 1,676$ & $1.7 \%$ \\
\hline $60^{\prime \prime}+$ & $\$ 2,789$ & $0.3 \%$ & $\$ 2,277$ & $2.5 \%$ \\
\hline $\begin{array}{c}\text { Volume-weighted } \\
\text { average }\end{array}$ & $\$ 609$ & - & $\$ 838$ & - \\
\hline
\end{tabular}

Source: Authors' calculations, DisplaySearch 2010g

Table 6-3. Average Market Price of LCD and PDP TVs in the U.S., ${ }^{\text {st }}$ Quarter 2010

In addition, historical prices are adjusted by the consumer price index (CPI) (U.S. Department of Labor, April 15, 2011), excluding the impact of inflation.

\subsubsection{Learning Rates}

Because a full historical data set for LCD and PDP TVs was not available, we assume that the first quarter of 2007 represents the initial production. Based on the data from 2007 to 2010 and the above equations, the learning rate for LCD TVs is approximately $14.6 \%$ and the rate for PDP TV is approximately $22.1 \%$. In other words, the learning rate of $14.6 \%$ indicates a $14.6 \%$ drop in price for a doubling in cumulative production of LCD TVs. Table 6-4 summarizes price learning rates for LCD TVs and PDP TVs. Figure 6-14 shows a graph corresponding to Table 6-4.

\begin{tabular}{l|c|c}
\hline Technology & LCD TVs & PDP TVs \\
\hline Trend & Price learning & Price learning \\
\hline b parameter & 0.2269 & 0.3599 \\
\hline Learning Rate (\%) & $14.6 \%$ & $22.1 \%$ \\
\hline Years Included & $2007-2010$ & $2007-2010$ \\
\hline Notes on Shipments & $\begin{array}{c}\text { Quarterly cumulative shipments of } \\
\text { LCD TVs in the global market }\end{array}$ & $\begin{array}{c}\text { Quarterly cumulative shipments of } \\
\text { PDP TVs in the global market }\end{array}$ \\
\hline Notes on Price Data & $\begin{array}{c}\text { Volume-weighted- and Inflation- } \\
\text { adjusted- DisplaySearch prices using } \\
\text { CPI }\end{array}$ & $\begin{array}{c}\text { Volume-weighted- and Inflation- } \\
\text { adjusted- DisplaySearch prices using } \\
\text { CPI }\end{array}$ \\
\hline
\end{tabular}

Table 6-4. Price Learning Rates for LCD and PDP TVs 


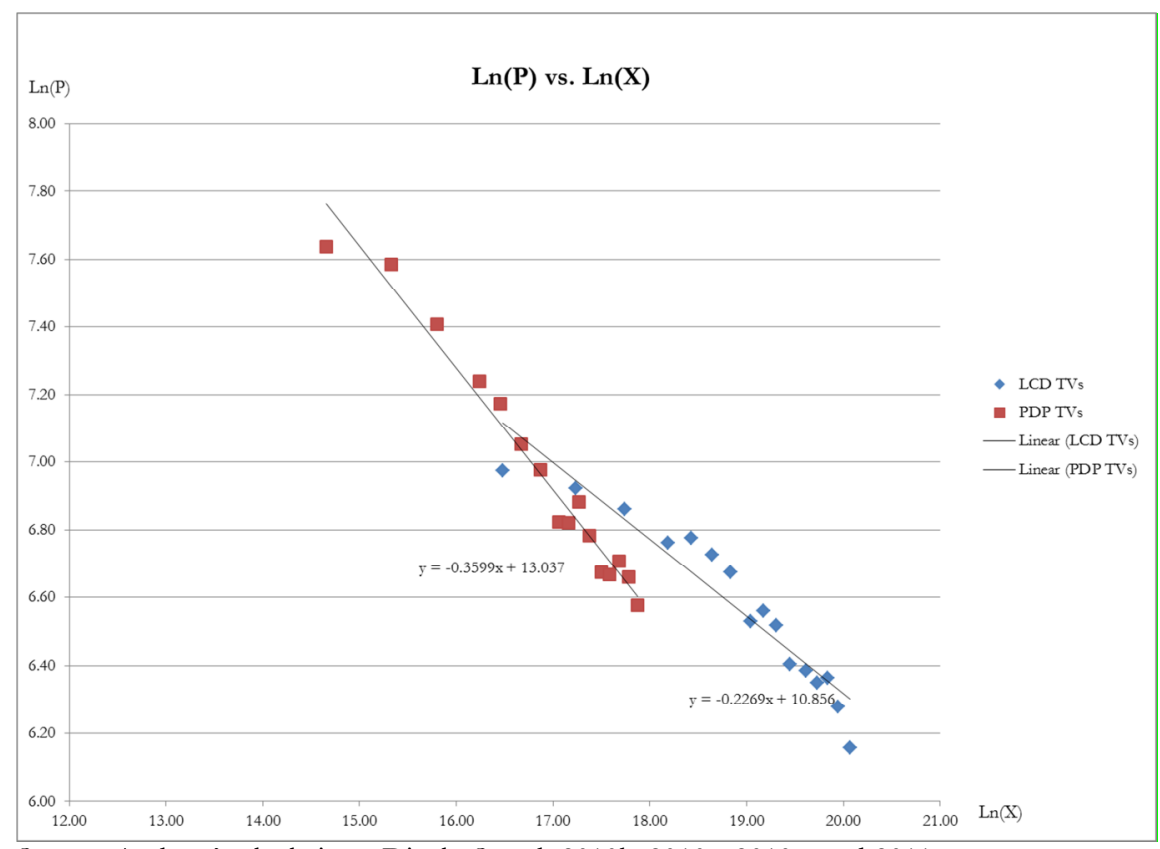

Source: Authors' calculations, DisplaySearch 2010b, 2010e, 2010g, and 2011a

Figure 6-14. Price Learning Rates for LCD and PDP TVs (2007 to 2010)

As seen in Figure 6-14, it is difficult to conclude that the relationship between LCD price and cumulative production is successfully matched with a linear function. In fact, assuming that the DisplaySearch forecast is very close to the actual values during the period from 2011 to 2014, b parameter (See Equation (ii)) of PDP TVs is slightly decreased while that of LCD TVs is increased. In other words, the learning rate for LCD TVs is expected to increase while the rate for PDP TVs is not. According to the results, the learning rate of LCD TVs for this case is $18.4 \%$ and that of PDP TVs is $20.7 \%$. Figure 6-15 shows the price learning rates including the forecast data from 2011 to 2014.

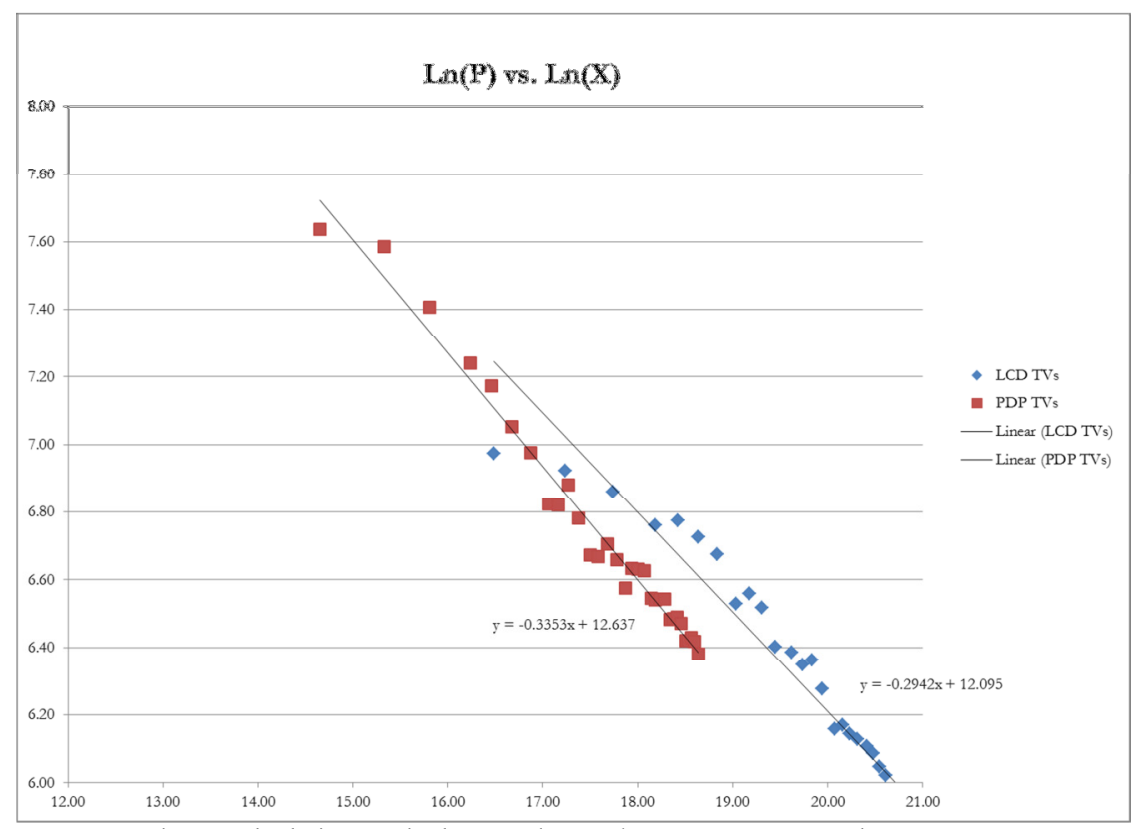

Source: Authors' calculations, DisplaySearch 2010b, 2010e, 2010g, and 2011a

Figure 6-15. Price Learning Rates Estimated for LCD and PDP TVs (2007 to 2014) 
Given that the global shipment of LCD TVs keeps increasing while that of PDP TVs is expected to remain nearly constant, this difference in learning curves seems reasonable.

\subsection{Standby Power}

The subsections below define standby power, and characterize TV standby power use of network TV as well as potential improvements.

\subsubsection{Definitions}

TV standby mode power consumption, typically $<1 \mathrm{~W}$, is much less than on-mode power, but it is necessary to consider total TV energy consumption in TVs, i.e., standby plus on-mode power. This report focuses mainly on screen technology efficiency improvement potentials, which are associated with on-mode power, but we briefly discuss TV standby mode power issues in this section. Before we discuss standby power in TVs, it is important to note that there are many definitions of low-power modes in devices. A recent report from APP\&IEA (APP\&IEA 2010) broadly categorizes product modes as active, low power, or disconnected. Table 6-5 describes these three modes.

\begin{tabular}{|c|c|c|}
\hline Modes & Descriptions & Relevant Terms \\
\hline Active/High Power & $\begin{array}{l}\text { "An active mode of a product is where one or } \\
\text { more primary functions }{ }^{71} \text { are operating or } \\
\text { activated." } \\
\text { - Some products, e.g., computers, can operate at } \\
\text { different levels in active mode, including "idle" } \\
\text { mode where the products is immediately ready to } \\
\text { be active but is doing nothing useful. }\end{array}$ & On, In-Use, Active, Idle \\
\hline Low Power & $\begin{array}{l}\text { In low-power mode, no primary function is } \\
\text { operating. } \\
\text { Network functions can be available depending on } \\
\text { particular product design. } \\
\text { - Many of network functions can be present in } \\
\text { active modes as well. }\end{array}$ & $\begin{array}{l}\text { Off, Standby, Active } \\
\text { standby, Passive standby, } \\
\text { Network standby, Sleep, } \\
\text { Idle, Fast start }\end{array}$ \\
\hline Disconnected & $\begin{array}{l}\text { Although in principle the product is using no } \\
\text { mains power when disconnected from all power } \\
\text { sources, some mobile devices with internal battery } \\
\text { power can remain active. }\end{array}$ & "Unplugged" \\
\hline
\end{tabular}

Source: APP\&IEA 2010

\section{Table 6-5. Power Mode Categories}

Our discussion of TV standby power will focus on low-power modes in TVs and uses the following concepts and definitions:

\section{Off Mode}

In general, "off mode" or "off mode with losses" represents the condition in which the product is connected to a power source but provides no user-oriented functions. The product cannot be remotely activated in this mode. This mode is different from "disconnected mode" or "unplugged mode." IEC 62087 (2011), IEC 62301 (2011), ENERGY STAR (2011b) and the International Standby Basket of Products project (2010), which is driven by the Australian Government's the Department of Climate Change and Energy Efficiency,

\footnotetext{
71 In general, primary functions encompass the intended purpose or use of the product - the main energy service the product provides, e.g., washing clothes for a clothes washer, and display of a picture with sound for a TV. Secondary functions are all other functions which can support the primary function or assist with the use and operation of the product. (APP\&IEA2010)
} 
provide the following definitions:

\begin{tabular}{|c|c|}
\hline Definitions of "Off Mode" & Source \\
\hline $\begin{array}{l}\text { "The appliance is connected to a power source, fulfills no function and } \\
\text { cannot be switched into any other mode with the remote control unit, an } \\
\text { external or internal signal." }\end{array}$ & IEC62087 Ed.3 draft, 2011 \\
\hline $\begin{array}{l}\text { "The energy using product is connected to a mains power source and is not } \\
\text { providing any standby mode, network mode or active mode function, and } \\
\text { the mode usually persists." }\end{array}$ & IEC62301 Ed.2, 2011 \\
\hline $\begin{array}{l}\text { "Off mode is a mode where the product is connected to a mains power } \\
\text { source and is not providing any On Mode or Sleep Mode functions, and } \\
\text { where the mode may persist for an indefinite time" }\end{array}$ & ENERGY STAR 2011b \\
\hline $\begin{array}{l}\text { "Off mode is when a product or appliance is connected to a power source } \\
\text { but does not produce any sound or picture, transmit or receive information } \\
\text { or is waiting to be switched "on" by the consumer. If the product has a } \\
\text { remote control, it cannot be woken by the remote control from off mode - } \\
\text { it can only be activated via the power switch on the product. No display } \\
\text { should be active in off mode. While the product may be doing some internal } \\
\text { functions in off mode (e.g., memory functions, EMC filters), these are not } \\
\text { obvious to users. An LED may be present to indicate off mode" }\end{array}$ & $\begin{array}{l}\text { International Standby } \\
\text { Basket of Products project, } \\
2010\end{array}$ \\
\hline
\end{tabular}

\section{Table 6-6. Definitions of Off Mode}

\section{Standby Mode}

In general, in the standby mode category, the product is on and connected to a mains power source but the product is not performing its main function. One or more of the following user-oriented or protective functions is available (APP\&IEA 2010, adapted from IEC 62301 Edition 2 final draft international standard):

- Function to facilitate the activation of other modes by remote switch, internal sensor, and timer

- Continuous function - information or status displays including clocks

- Continuous function - sensor-based functions

According to the EuP Preparatory Study Lot 6 (Fraunhofer 2007(2)a), there are three basic approaches for defining standby that either do or do not include off modes:

- The lowest-power mode of a product (including $0 \mathrm{~W}$ )

- A mode when the product is using energy, but no main function is running

- A mode when a product is "not effectively delivering" a main function to a user or a connected system ("idle losses")

Within standby mode, further subdivisions such as "passive standby" and "active standby" are defined. In "passive standby," the energy-using product is off but can be powered up remotely, e.g., as can be done for TVs, VCRs, and audios. This also may include basic clock and memory functions (APP\&IEA 2010). In "active standby," the energy-using product is on but does not provide primary functions, e.g., as is the case for set-top boxes (Kim 2010). IEC 62087 (2011) and The International Standby Basket of Products project (2010) provide following definitions: 


\begin{tabular}{|c|c|}
\hline Definitions of Active Standby / Passive Standby & Source \\
\hline $\begin{array}{l}\text { Standby-passive: "The appliance is connected to a power source, produces } \\
\text { neither sound nor picture but can be switched into another mode with the } \\
\text { remote control unit or an internal signal" }\end{array}$ & IEC62087 Ed.3 draft, 2011 \\
\hline $\begin{array}{l}\text { "Passive standby is when a product or appliance is not performing its } \\
\text { main function (sleeping) but it is ready to be switched on (in most cases with } \\
\text { a remote control) or is performing some secondary function (e.g., has a } \\
\text { display or clock which is active in this mode). This mode also applies to } \\
\text { power supplies for battery operated equipment (portable appliances which } \\
\text { are intended to be used when disconnected from the base station) when the } \\
\text { appliance is not being charged (disconnected)." }\end{array}$ & $\begin{array}{l}\text { International Standby } \\
\text { Basket of Products project, } \\
2010\end{array}$ \\
\hline $\begin{array}{l}\text { Standby-active, low: "The appliance can additionally be switched into } \\
\text { another mode with an external signal." } \\
\text { Standby-active, high: "The appliance is exchanging/ receiving data } \\
\text { with/from an external source." }\end{array}$ & IEC 62087 Ed.3 draft, 2011 \\
\hline $\begin{array}{l}\text { "Active standby is when the appliance is on but not performing its main } \\
\text { function. This mode is usually present in devices (a) where there is a } \\
\text { mechanical function which is not active, e.g., DVD drive to motor, but } \\
\text { where power circuits are on, or (b) where a device has a battery and the } \\
\text { device is charging." }\end{array}$ & $\begin{array}{l}\text { International Standby } \\
\text { Basket of Products project, } \\
2010\end{array}$ \\
\hline
\end{tabular}

Table 6-7. Definitions of Active Standby and Passive Standby

"Sleep Mode" is not universal, but it can be defined as a mode where the energy-using product switches off some functions after a certain period of inactivity or by manual selection (Kim 2010). "Sleep Mode" is sometimes the same as or a subset of standby mode. Whether this mode is "off" depends on the definition of "off mode." ENERGY STAR's TV requirements have been using "sleep mode" instead of "standby" with the same concepts described above, and defines it as follows:

- "Sleep Mode is defined as the time when the product is connected to a power source, produces neither sound nor picture, neither transmits nor receives program information and/or data (excluding data transmitted to change the unit's condition from Sleep Mode to On Mode), and is waiting to be switched to On Mode by a direct or indirect signal from the consumer, e.g., with the remote control." (ENERGY STAR 2011b)

Network Standby Modes can be defined as any mode where network function(s) are activated but the primary function is not activated (APP\&IEA 2010). Products in network standby mode can wake in response to information over the network. According to the EuP Preparatory Studies Lot 26 (Fraunhofer 2010a), networked standby modes are defined as "conditions in which the equipment provides reduced functionality, but retains the capability to resume applications through a remotely initiated trigger via network connection."

Although there have been numerous definitions and proposals for definitions of power modes, detailed discussion is out of scope of this report. In addition, modes vary within a product as well as across product types. Powered devices originally had only two basic states: on/off. Now there are many intermediate modes between on and off with different functionalities and power levels. In particular, increasing network connectivity makes it more complex to define low-power modes in appliances. Although it is expected that the power modes of the future will be possibly categorized as "on," "sleep" (including network connectivity), and "off," it is important to note these terms are not yet harmonized globally.

\subsubsection{Standby Mode (Sleep Mode) Power of TVs}

Currently, a majority of TVs consume close to or less than $1 \mathrm{~W}$ in standby, mostly passive standby. U.S. 
ENERGY STAR requires that measured power $\left(\mathrm{P}_{\mathrm{s}}\right)$ of a TV in sleep mode be less than or equal to $1.0 \mathrm{~W}$. The simple average of sleep mode power of ENERGY STAR-qualified LCD and PDP TVs is $0.33 \mathrm{~W}$ (ENERGY STAR 2011). About $70 \%$ of the TVs consume less than $0.5 \mathrm{~W}$ in sleep mode (Figure 6-16). Sleep mode power consumption does not depend on screen size (Figure 6-17), and the average standby power of TVs listed in ECCJ is in line with the trend shown by ENERGY STAR TVs (Figure 6-18).

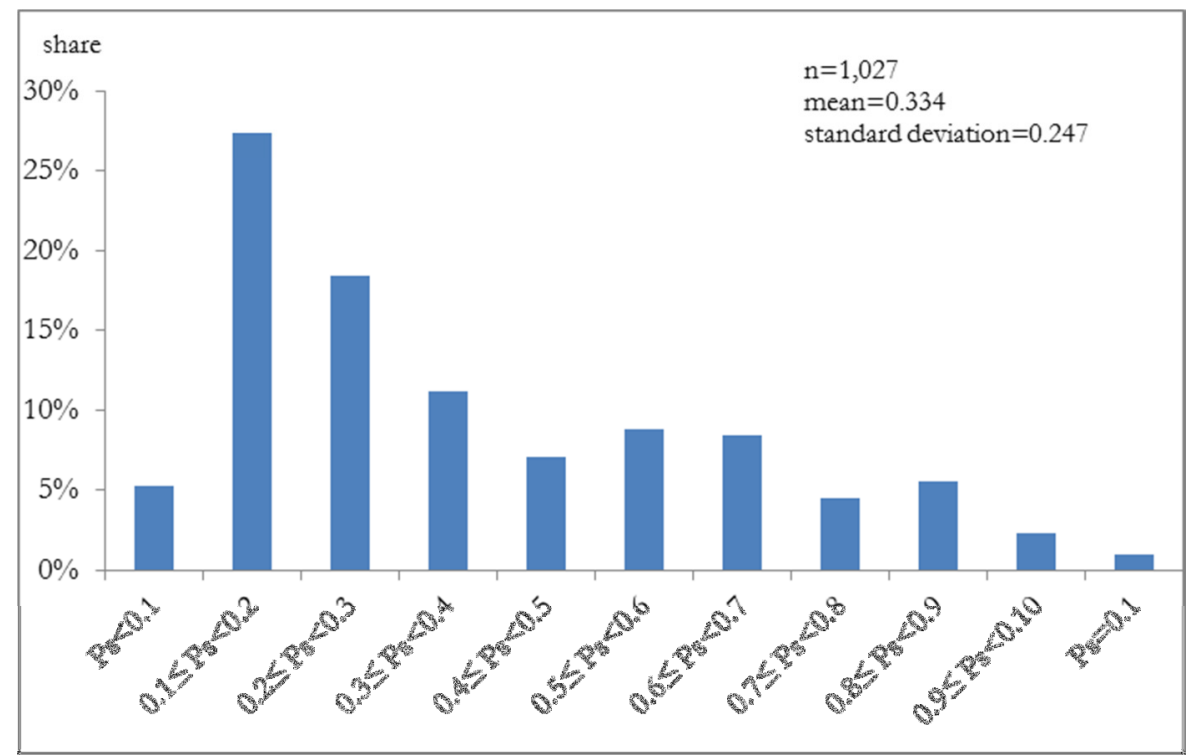

Figure 6-16. Distribution of Sleep Mode Power of ENERGY STAR-qualified LCD and PDP TVs

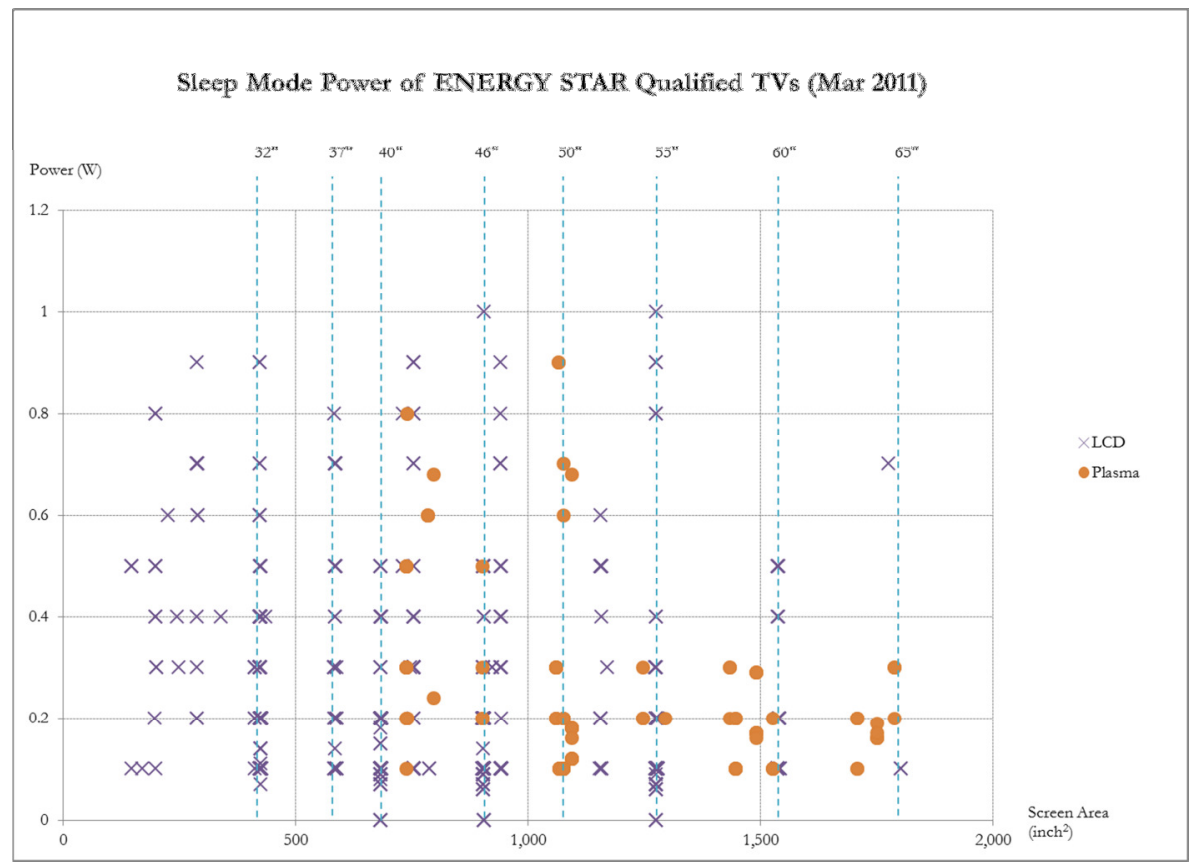

Figure 6-17. Sleep Mode Power of ENERGY STAR-qualified TVs by Screen Area 


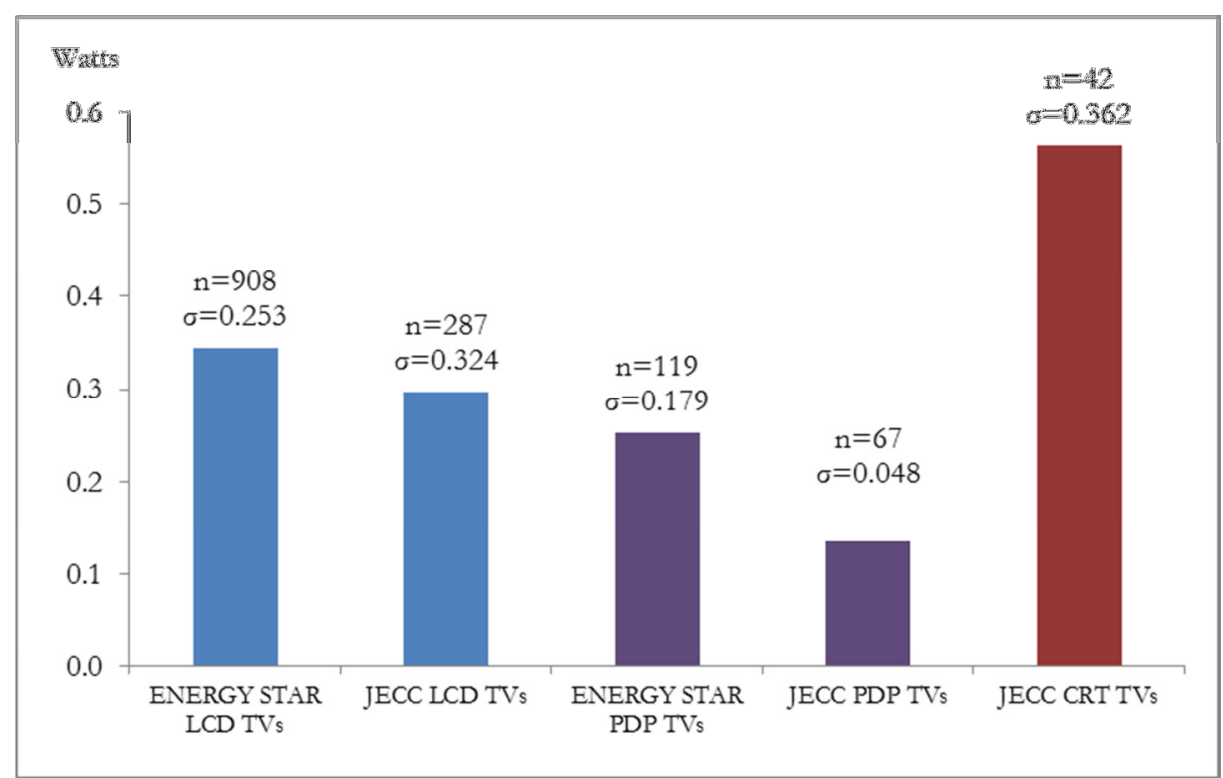

$*$ n: number of TV models, $\sigma=$ standard deviation

Figure 6-18. Average Sleep Mode Power of TVs in Japan and the U.S.

\section{Consumption in Standby Mode (Sleep Mode)}

Because standby power does not depend on screen size, energy consumption in standby mode is relatively simple to estimate compared to consumption in on mode. Assuming that the average standby power of ENERGY STAR TVs represents all TVs across the globe and that the average worldwide daily on-time usage is 4 hours while the remaining 20 hours are spent in standby mode, then the total energy consumption in standby mode of new TV sales can be estimated as described below. Total TV sales (for CRT, LCD, and PDP TVs only) are expected to increase by 16\% from 247 million units in 2010 to 287 million units in 2014 (DisplaySearch 2011a), but annual energy consumption in standby mode is expected to increase by $6.6 \%$ from $671 \mathrm{GWh}$ in 2010 to $715 \mathrm{GWh}$ in 2014 because LCD TVs consume less power in standby than traditional CRT TVs (Figure 6-19).

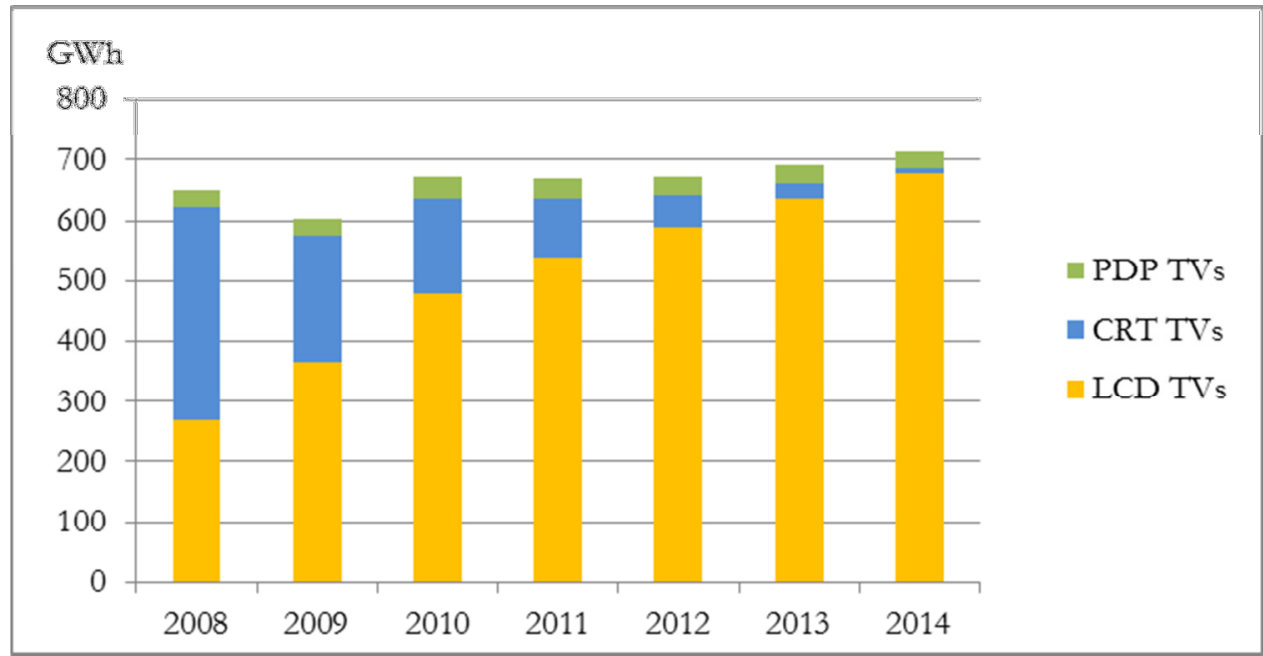

Figure 6-19. Estimates of Energy Consumption in Sleep Mode by Global TV Sales

If we apply the average sleep mode power of ENERGY STAR LCD and PDP TVs and the average standby power of CRT TVs in Japan to all other TVs sold in other countries, annual energy consumption per unit in each country is as shown in Table 6-8. The results indicate that annual energy consumption per TV in standby 
mode is not significant compared to energy consumption in on mode.

\begin{tabular}{|c|c|c|c|c|c|c|c|c|}
\hline \multirow[b]{2}{*}{ Country } & \multirow[b]{2}{*}{$\begin{array}{l}\text { Hours of } \\
\text { Usage } \\
\text { per Day } \\
\text { (On Mode) }\end{array}$} & \multirow[b]{2}{*}{$\begin{array}{c}\text { Hours of } \\
\text { Non-Usage } \\
\text { per Day } \\
\text { (Standby) }\end{array}$} & \multicolumn{2}{|c|}{ LCD } & \multicolumn{2}{|c|}{ PDP } & \multicolumn{2}{|c|}{ CRT } \\
\hline & & & $\begin{array}{l}\text { On Mode } \\
\text { UEC } 2012\end{array}$ & $\begin{array}{l}\text { Sleep Mode } \\
\text { UEC } 2012\end{array}$ & $\begin{array}{l}\text { On Mode } \\
\text { UEC } 2012\end{array}$ & $\begin{array}{l}\text { Sleep Mode } \\
\text { UEC } 2012\end{array}$ & \begin{tabular}{|l} 
On Mode \\
UEC 2012
\end{tabular} & $\begin{array}{l}\text { Sleep } \\
\text { UEC } 2012\end{array}$ \\
\hline & hrs & hrs & $\mathrm{kWh} / \mathrm{yr}$ & $\mathrm{kWh} / \mathrm{yr}$ & $\mathrm{kWh} / \mathrm{yr}$ & $\mathrm{kWh} / \mathrm{yr}$ & $\mathrm{kWh} / \mathrm{yr}$ & $\mathrm{kWh} / \mathrm{yr}$ \\
\hline Australia & 6.5 & 17.5 & 111.5 & 2.20 & 237.1 & 1.62 & 110.8 & 3.60 \\
\hline Canada & 4.5 & 19.5 & 90.0 & 2.46 & 190.5 & 1.80 & & \\
\hline China & 4 & 20 & 76.3 & 2.52 & 145.3 & 1.85 & 74.2 & 4.12 \\
\hline Europe & 4 & 20 & 64.4 & 2.52 & 146.5 & 1.85 & & \\
\hline India & 3.5 & 20.5 & 60.1 & 2.58 & 127.7 & 1.89 & 59.7 & 4.22 \\
\hline Japan & 4.5 & 19.5 & 73.2 & 2.46 & 158.0 & 1.80 & & \\
\hline Korea & 6 & 18 & 103.0 & 2.27 & 218.9 & 1.66 & 102.3 & 3.80 \\
\hline Mexico & 4 & 20 & 70.1 & 2.52 & 143.7 & 1.85 & 70.9 & 4.22 \\
\hline Russia & 4 & 20 & 68.7 & 2.52 & 140.1 & 1.85 & 71.1 & 4.22 \\
\hline South Africa & 4 & 20 & 70.2 & 2.52 & 141.6 & 1.85 & 69.8 & 4.22 \\
\hline US & 5 & 19 & 100.0 & 2.39 & 211.6 & 1.75 & & \\
\hline
\end{tabular}

Table 6-8. Estimates of TV Unit Energy Consumption (UEC) in On-Mode and Standby Mode

\subsubsection{Recent Trends of Standby Power in 'TVs}

Recent TVs have many new features, including network connectivity, hard disks, DVD recorders, and digital tuners. With these additional features, these products are often required to have fast boot times. In particular, connected TVs (or smart TVs) are, because of their integrated features, likely to consume more energy in standby mode than current conventional TVs do.

\section{A] Fast Start Mode}

Although most TVs feature very low standby mode power, much less than $1 \mathrm{~W}$, one issue of current TVs is booting (or reactivating) time which can increasingly feature idle modes ("fast play", "quick start") that allow the TV system to power up within a few seconds from these modes. According to the EuP Preparatory Study, fast-start options consume more than $25 \mathrm{~W}$ in standby for complex TVs (Fraunhofer 2010b). According to a recent report from ECCJ (ECCJ 2009), fast start options contribute about $25 \mathrm{~W}$ on average (Min 11.7 W, Max $68.0 \mathrm{~W}$ ) to standby power consumption in a TV. As the power-up time can vary depending on the actions required to boot (or reactivate) devices, slow boot (or wake) time will suggest that consumers will be likely to choose a fast start mode although the mode consumes more power than the default standby. Table 6-9 shows power consumption for fast start modes in TVs.

\begin{tabular}{r|r|r|r|r|r}
\hline & \multicolumn{1}{|c|}{$\mathrm{n}$} & Average & $\max$ & \multicolumn{1}{c}{$\min$} \\
\hline & TVs & 50 & 24.45 & 68.00 & 11.70 \\
\hline CRT & 2 & 11.70 & 11.70 & 11.70 \\
\hline LCD & 48 & 24.99 & 68.00 & 11.70 \\
\hline PDD & - & - & - & - \\
\hline
\end{tabular}

Source: ECCJ 2009, unit: Watts

\section{Table 6-9. Power Consumption in Fast Start Mode of TVs}

While it is not expected that the maximum level of power consumption in the fast start mode will increase, energy consumption in the mode (as a standby mode) will be significant once it is chosen by the user, as recent smart TVs (connected TVs) may drive up these functions. However, it is difficult to precisely forecast additional energy consumed in the fast start modes of TVs because sufficient market data and consumer usage data are not available at the time of writing. Assuming that $10-30 \%$ of LCD TVs ( $\geq 30$ inches) in the 
market are/will be operated in fast start mode (average $25 \mathrm{~W}, 20$ hours per day), instead of normal passive standard mode, the annual energy consumption in the fast start mode of TVs (potentially to be sold) in 2014 is estimated to be about $3.9-11.6 \mathrm{TWh}$.

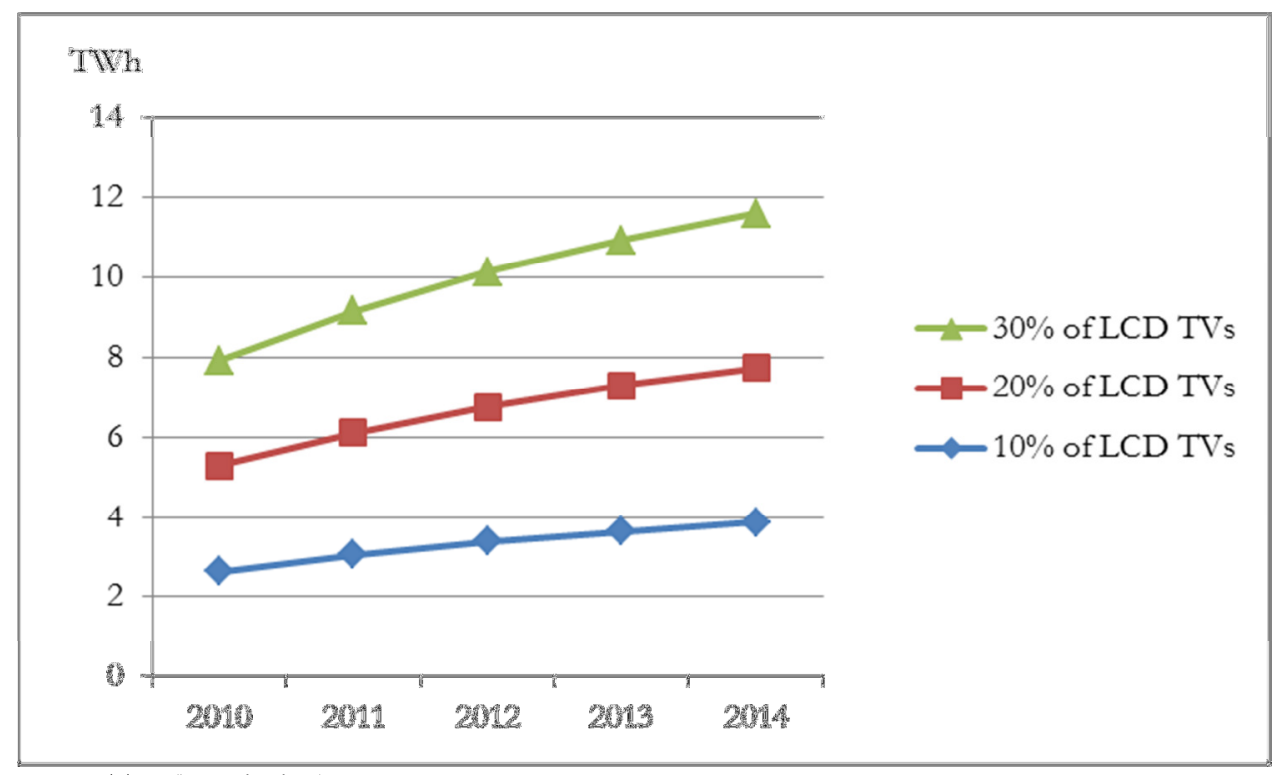

* LCD TVs $(\geq 30$ inches)

\section{Figure 6-20. Estimates of Energy Consumption in Fast Start Mode by Global TV Sales}

Although fast start mode is not necessarily a network-related feature, the fast start options coincide with additional network connectivity for recent connected TVs (smart TVs). Boot time (or wake time) depends mainly on memory size, number of device drivers, and number of applications that are closely associated with smart TVs (connected TVs).

\section{B] Data Acquisition Mode}

Active standby mode in TVs is used to perform downloads of Electronic Program Guide (EPG) and firmware updates. As described in Table 6-7 above, Active standby (high) is defined as "the appliance is exchanging/ receiving data with/from an external source." (IEC 62087 Ed.3 draft, 2011) ENERGY STAR also defines Download Acquisition Mode (DAM) in TVs as below.

"The power mode in which the product is connected to a mains power source, produces neither sound nor picture, and is actively downloading data. Data downloads may include channel listing information for use by an electronic programming guide, TV setup data, channel map updates, firmware updates, monitoring for emergency messaging/communications or other network communications."

(ENERGY STAR 2011b)

According to the ECCJ report (ECCJ 2009), LCD TVs, Plasma TVs, and Blu-ray recorders consume more than $10 \mathrm{~W}$ in data acquisition modes, and spend from a few minutes to one hour per day in the mode. The power consumption in the mode varies with HDMI connection, digital double tuner, stable signal reception, etc. According to the report, data acquisition mode consumes $21 \mathrm{~W}$ on average (Min $9.5 \mathrm{~W}$, Max $41 \mathrm{~W}$ ). Table 6-10 shows power consumption in data acquisition modes of TVs. 


\begin{tabular}{r|r|r|r|r|r}
\hline & \multicolumn{1}{|c|}{$\mathrm{n}$} & \multicolumn{1}{c|}{ Average } & \multicolumn{1}{c}{$\max$} & \multicolumn{1}{c}{$\min$} \\
\hline & TVs & 51 & 20.85 & 41.00 & 9.50 \\
\hline CRT & - & - & - & - \\
\hline LCD & 37 & 20.36 & 41 & 9.50 \\
\hline PDP & 14 & 22.14 & 27 & 18.00 \\
\hline \multicolumn{2}{|c|}{ DVD $\cdot$ Video·HDD Built-in TVs } & 6 & 31.83 & 34.00 & 31.00 \\
\hline
\end{tabular}

Source: ECCJ 2009, unit: Watts

Table 6-10. Power Consumption in Data Acquisition Mode of TVs

Assuming that a TV spends $0.5-3$ hours in the data acquisition mode, consuming $20 \mathrm{~W}$ on average, the daily energy consumption in the mode is from $0.01 \mathrm{kWh}$ to $0.06 \mathrm{kWh}$. Average energy consumption in DAM of ENERGY STAR-qualified TVs is in a range of 0 to $0.06 \mathrm{kWh}$ per day (maximum allowable energy in DAM: $0.08 \mathrm{kWh}$ per day for ENERGY STAR Version 4). However, it is difficult to forecast additional energy consumed in these data acquisition modes of TVs because sufficient market data are not available. Assuming that 10-30\% (10-50\% from 2012 to 2014) of LCD and PDP TVs ( $\geq 30$ inches) in the market are/will be used in data acquisition mode (average $20 \mathrm{~W}, 2$ hours per day), the annual energy consumption in the mode of TVs (potentially to be sold) in 2014 is estimated to be about $0.3-1.0 \mathrm{TWh}$.

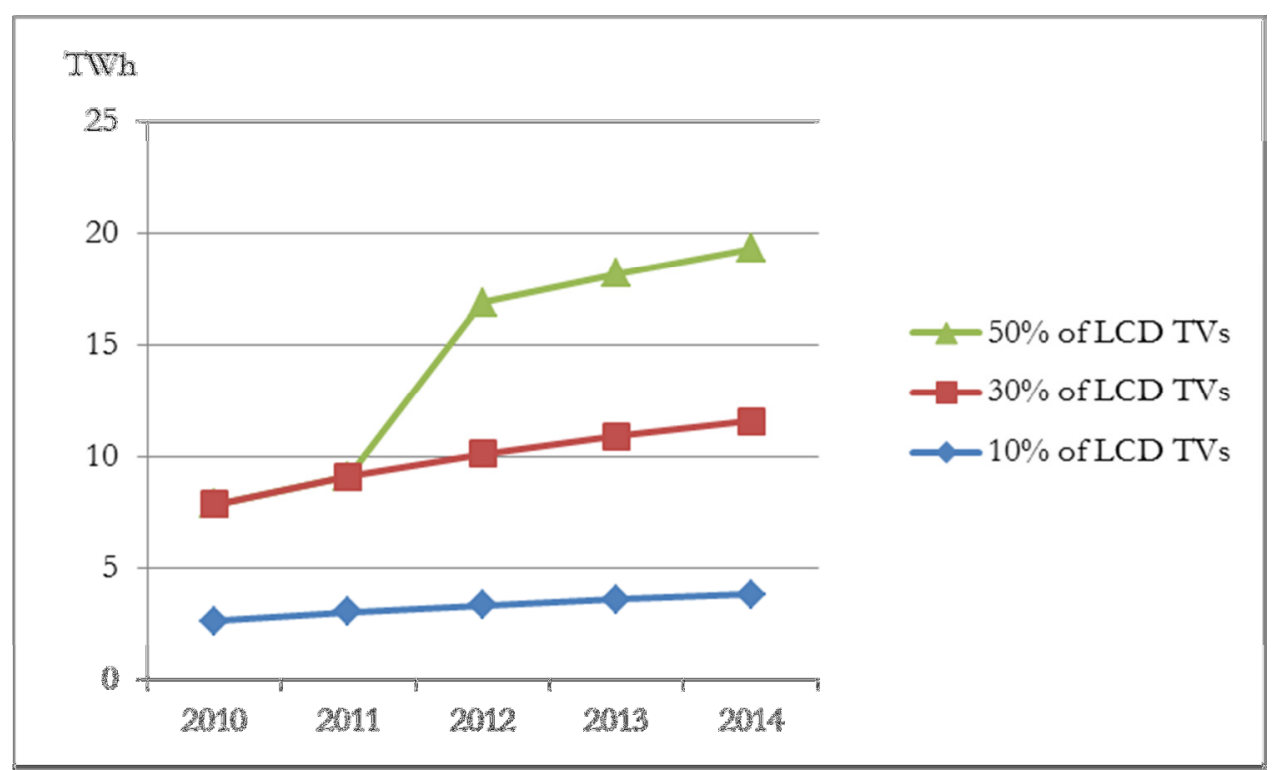

Figure 6-21. Estimates of Energy Consumption in Data Acquisition Mode by Global TV Sales

According to ENERGY STAR Version 5 requirements (ENERGY STAR 2011b), measured DAM energy consumption for all DAM states (E $\left.\mathrm{E}_{\mathrm{DAm}}\right)$ should be less than or equal to 40 watt-hours per day ( $0.04 \mathrm{kWh} /$ day) which is half of the ENERGY STAR Version 4 requirement $(0.08 \mathrm{kWh} /$ day $)$. While power consumption for both fast start mode and data acquisition mode is about 20-25 W on average, energy consumption consumed in the fast start mode, if selected, is expected to be much higher than that in data acquisition mode because in the length of time the fast start mode is active. Also, energy-efficiency standards have been including and updating power requirements for data acquisition modes.

\section{C] Network Standby Mode}

In general, there are two approaches for network connectivity in TVs. In the first, the TV is Internet ready, and users can access content from the internet, such as streaming movies from services after making a 
physical Ethernet or Wi-Fi connection to their home network. In the second, the TV has a set-top box or Conditional Access, e.g., CableCARD, hardware incorporated. This enables users to easily access the internet from their TVs via the cable or satellite network, possibly without the need for a separate Ethernet connection to their home network. However, the currently effective energy-efficiency test methods and regulations do not consider network connectivity, and energy consumption testing is done with the TV not connected to the internet or network, in spite of a possibility of more power consumption in such cases than power consumption in no-network standby mode. Traditionally, policies to reduce energy consumption for stand-alone products in low-power modes have used the strategy of including a requirement that power management for a device enter the relevant low-power mode under specified conditions. However, setting limits on low-power modes may not be effective for networked products if the products never enter those modes (APP\&IEA 2010).

A connected TV is basically performing a subset of what a personal computer (PC) does when it is in sleep mode. Thus, a TV's network standby power should be no more than a PC's power consumption in sleep mode, which is usually in a range of 2 to 4 W (ENERGY STAR 2011d). In fact, the minimum power requirement for basic network processing seems to vary among TV manufacturers because it depends on the TV's internal design scheme and specifications. In addition, it is necessary to consider both power mode and resume-time-to-application (e.g., boot time or wake time) in order to accurately estimate network TV standby power consumption because a standby mode that permits fast boot time (or wake time) typically requires more power than a standby mode with slower boot time (or wake time).

However, several factors make it difficult for the authors to fully analyze energy consumption for connected TVs (smart TVs) in this report: 1) lack of data on network standby power and fast start options in recent TVs, 2) the rapid evolution in network technology, and 3) uncertain effect of changes in user behavior with network TV products. Moreover, if TV power consumption in network standby mode is regulated, it is possible for TV manufacturers to get their products to PC standby power consumption levels. Although this report does not fully analyze network standby issues in TVs, we estimate power consumption of connected TVs (smart TVs) in network standby mode based on the following information:

\section{- Connected TV Market}

According to DisplaySearch (2011e), the connected TV market share will increase from 44 million units in 2010 to 123 million units in 2014 when it will account for about $43 \%$ of the total TV market.

- Boot Time (or Reactivation Time)

According to a review from CNET (CNET 2010), the Quick Start mode of Sony Google TV (NSXGT1) series enables the models to wake up in about 4 seconds by consuming $24 \mathrm{~W}$ of standby power, while it takes about 45 seconds to power up Google TV normally by consuming $0.14 \mathrm{~W}$. According to the EuP Preparatory Study (Fraunhofer 2010d), a recent Sony TV model (KDL-52LX905) consumes $20 \mathrm{~W}$ in fast-reactivation mode, while it consumes $0.2 \mathrm{~W}$ in standby mode. Fast start mode is not necessarily a network-related feature, but the fast start option coincides with additional network connectivity. The EuP Preparatory Study (Fraunhofer 2010b), provided an example that describes the relationship.

"An example would be a TV display in the bed room that is wireless connected to a main TV receiver in the living room. The receiver box would get a wake-up signal via a WiFi adapter/router. This type of solution requires more energy than regular standby as the TV/AV receiver provides such network wake-up typically only out of a bigher power state. The so called "Fast Play" or "Quick Start" options that are provided by some manufacturers for media player/recorder or complex TV s consume from 8 watts to over 25 Watts in "bot" standby. In conclusion we could assume that a large group of products that are currently feature $<1 W$ standby may increase energy consumption for faster resume time to application." (Fraunhofer 2010b) 


\section{- Standby Power}

Based on the above information, we assume that although a TV's network standby power consumption should in principle be similar to a PC's sleep mode power consumption, i.e., about 2 to $4 \mathrm{~W}$, a connected TV can consume as much as $30 \mathrm{~W}$ in standby mode, depending on the manufacturer's design scheme and various options for fast reactivation times and additional features.

- Power Management Regime

Connected TVs are expected to employ power management regimes. For example, because TV viewing is a passive activity where the user interacts with the set only occasionally, it is possible that the TV set could be turned from on mode to a low-power state after a given period with no interaction from the user.

- Average Daily Hours used in Network Standby Mode

Depending on the power management regime, a connected TV's power usage in network standby mode will vary, which makes it difficult to analyze in this report.

\begin{tabular}{r|r|r|r}
\hline & 2010 & 2012 & \multicolumn{1}{c}{2014} \\
\hline Total TV Shipment (millions: M) & $243.5 \mathrm{M}$ & $263.3 \mathrm{M}$ & $287.8 \mathrm{M}$ \\
\hline Connected TVs (millions: M) & $43.9 \mathrm{M}$ & $87.2 \mathrm{M}$ & $122.7 \mathrm{M}$ \\
(market share) & $(21.1 \%)$ & $(33.1 \%)$ & $(42.6 \%)$ \\
\hline Network standby power (W) & $3-30$ & $3-30$ & $3-30$ \\
\hline Annual Energy Consumption in & $1.0-9.6$ & $1.9-19.1$ & $2.7-26.9$ \\
\hline Network Standby (TWh) & $\begin{array}{r}1.0-9.6 \\
(2010)\end{array}$ & $\begin{array}{r}4.3-42.8 \\
(2010-2012)\end{array}$ & $\begin{array}{r}9.3-92.6 \\
(2010-2014)\end{array}$ \\
\hline Cumulative Energy & Consumption (TWh) & &
\end{tabular}

Source: DisplaySearch 2011e and author's assumption for network standby power

\section{Table 6-11. Connected TV Market Forecast and Network Standby Power}

We assume that the average network standby power, including fast start option, of a connected TV ranges between $3 \mathrm{~W}$ and $30 \mathrm{~W}$. For example, a normal power management regime without fast reactivation requirement may consume $3 \mathrm{~W}$ at a minimum level, while a power management regime with fast reactivation requirement may consume up to $30 \mathrm{~W}$. Although the maximum power required for network connectivity and fast reactivation time, is not expected to significantly increase because of technological improvements, the average network standby power is expected to vary depending on the power management regimes applied. However, because it is difficult to forecast the average network standby power and daily hours in standby mode with a power management regime, we estimate the total energy consumption in network standby power within a possible range. 


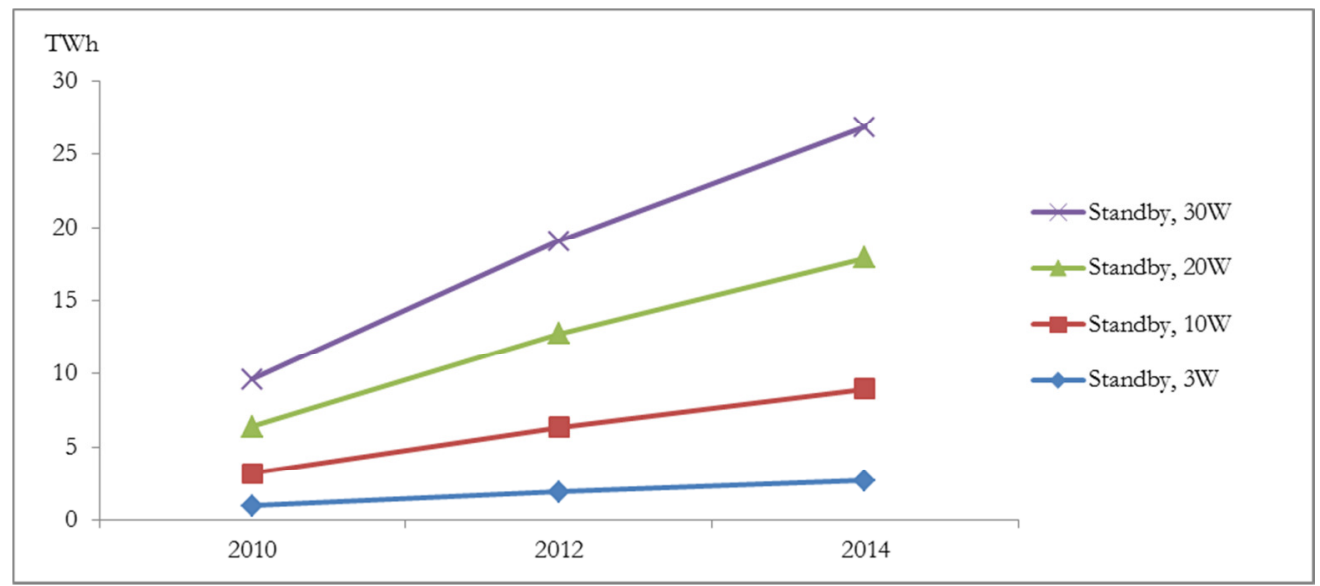

Source: Author's calculation

* Daily usage in network standby mode is assumed to be 20 hours.

\section{Figure 6-22. Estimates of Energy Consumption in Network Standby Mode by Global Connected TV Sales}

\subsubsection{Efficiency Improvement Options in Network Standby and Fast Start Mode}

To discuss efficiency improvement options for network standby power, it is necessary to address network technologies and relevant factors. This section discusses some examples briefly because details of network communication technologies are not a primary topic of this report.

\section{Data Link}

Energy-Efficient Ethernet (IEEE ${ }^{72}$ 802.3az) is a recently approved standard that switches rapidly between the full operating speed and the low-power idle (LPI) mode (Fraunhofer 2010b). Energy-Efficient Ethernet is applied to all equipment using common wired Ethernet protocols, primarily in local area networks (LANs). In addition, IEEE standard 802.11 specifies wireless local area network (WLAN, or Wi-Fi), one of the most common technologies for wireless network communication. This technology can be applied to other consumer electronics including TVs. According to an APP\&IEA 4E report (2010), there are a number of energy saving approaches for various WiFi protocols.

High-Definition Multimedia Interface (HDMI) is the most common A/V interfaces for transmitting uncompressed digital data for TVs, AV receivers, set-top-boxes, media players/recorders, PCs, etc. The HDMI 1.4a specification released in 2010 enables high speed bi-directional communication, IP-based applications, 3D and high resolution support, HDMI Ethernet channel, Digital Living Network Alliance (DLNA), Universal Plug and Play (UPnP), and Multimedia over Coax Alliance (MoCA) over a single cable. (Fraunhofer 2010b) Consumer Electronics Control (CEC) defined in the HDMI specification is a one-wire bi-directional serial bus that allows the user to command and control multiple CEC-enabled boxes with one remote control for networked devices to communicate with one another. In the long term, this concept is a valuable approach to energy saving in that devices manage their own power state, and HDMI is becoming more common in the AV product market. However, it is only available within a network of fully digital products connected via HDMI interfaces, and it seems to require mandatory, rather than voluntary, extensions to the HDMI standard in order to be effectively implemented. (APP\&IEA 2010)

\section{Power Management}

Many devices with Ethernet links support power management functions such as wake-on-LAN (WoL) and some Wi-Fi devices support wake-on-wireless LAN (WoWLAN), similar to wake-on-LAN for Ethernet.

72 Institute for Electrical and Electronic Engineers 
These enable a remote device to wake up, or they turn on a device that is asleep or off. However, the device is not "on the network" for ordinary network purposes and basic internet protocols (e.g., address resolution protocol (ARP) for internet protocol version 4 (IPv4). Network proxying is a technology that allows another network agent to act as a proxy for the sleeping device, e.g., a computer, set-top box, printer, or TV. Although many aspects of network "presence" are available through the proxy agent, the host can be in a low-power mode and can only be activated when needed. In particular, network proxying allows a router, i.e., access point, to act as a proxy for the sleeping client and enables the sleeping client to wake when network requests happen. The proxy can also be located in another edge device on the network (e.g., another PC), or can be internal to the sleeping device, either in or nearby the network interface hardware (Fraunhofer 2010b). This use of a proxy agent can also be used to help save network standby mode power consumption by connected TVs.

\section{Inter-Device Power Control}

As audio/video (A/V) devices become interconnected and complex, it is important to develop technological standards to manage their power states and incorporate solutions into future appliances and communication. Effective solutions for A/V inter-device power management need to embrace device autonomy, self-control, and standard behaviors (Nordman 2011). According to Nordman, a successful precedent is monitor power management with computers, and a power management regime similar to those for other devices can be developed. Here are some examples where a TV is included in the system:

Automatic actions are underlined.

a) A TV is powered up, and a DVD player is selected as the source; this causes the DVD player to wake and start its menu. The user selects "play" and begins watching a movie.

b) The TV is later shifted to broadcast (e.g., news) in the midst of the DVD player operation (which then pauses). The TV does not display the DVD signal for 15 minutes, so the DVD player goes to sleep.

c) Later, the movie that was previously being watched finishes, and then the DVD player shifts to its menu. After 15 minutes with no user input, the DVD player goes to sleep, which causes the TV to also go to sleep.

d) Later the TV is shifted to getting content from a set-top box, which causes the set-top box to wake. The set-top box delivers the content via an analog connection that does not allow it to know the power state of the TV. Four hours pass with no user interaction to the set-top box, so it overlays a message of imminent power down for 5 minutes, then goes to sleep. If the TV is not already asleep, it also goes to sleep based on the lack of signal.

These examples highlight key aspects of an appropriate solution for inter-device power management. Devices in these examples:

- Expose power state over the network, i.e. whether the device is fully on, or asleep

- Expose functional state over the network, e.g. whether data streams are actually being consumed, whether a media source is loaded (e.g. DVD or iPod), and time since last user input

- Establish default device behavior, including time delays suitable to human expectations

- Take into account power and functional information from other devices

- Go into a sleep state rather than to off as the normal low-power state

Accomplishing the above scenario is not simple and will require time. A comprehensive solution will require a set of interoperability standards that cross multiple data and user interface types. Although reliable figures on incremental costs and energy savings related to this option are not available yet, it is expected that savings by such automatic power control between $\mathrm{A} / \mathrm{V}$ devices will be very cost effective. 


\section{Boot Time and Resource Consumption}

According to a study (Jo, et al. 2010), for current digital TVs it takes 6-15 seconds ${ }^{73}$ for the first screen image to appear. This latency comprises of operating system (OS) booting, initializing hundreds of hardware registers $^{74}$, and launching applications. In other words, depending on actions required to boot and devices to initialize, the required startup time can vary, although it can be reduced by optimizing resource utilization. Figure 6-23 shows an example of startup time and resource utilization of a digital TV system.

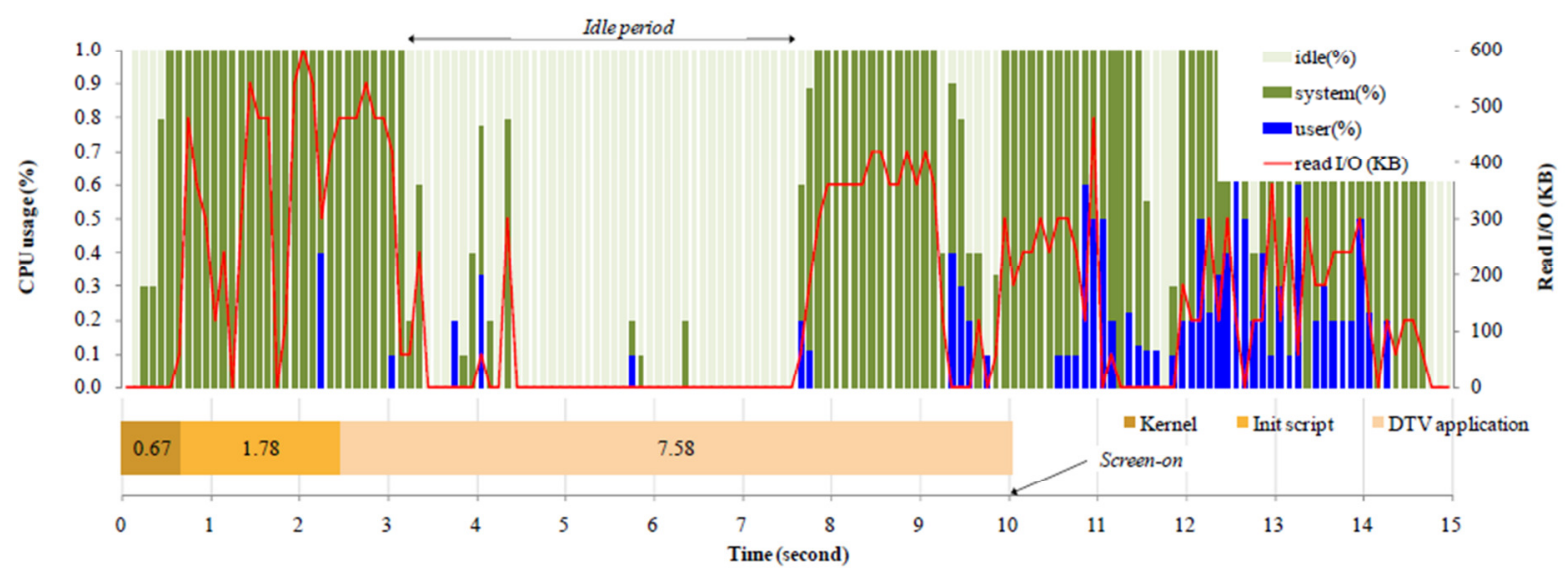

Source: Jo et al. 2009

\section{Figure 6-23. Startup Time and Resource Utilization of Digital TV System}

As boot time depends on memory size and number of device drivers and application, reduction in boot time (or wake time) for a smart TV (a connected TV) requires additional standby power, e.g. by refreshing random access memory (RAM), ensuring that the TV system runs in a different low power mode. Thus, it is possible that smart TVs (connected TVs) will increasingly feature such idle modes of fast play or quick start as user selected options. While all types of TVs have been operated on Linux OS so far, some recent smart TVs (or connected TVs) are designed on Android OS. Improvement in booting technologies, operated on both systems and independent of memory size, is key to energy-efficiency improvement in fast start mode. Although there are technological options to improve boot time in embedded system such as TVs, analysis of them is beyond the scope of this report.

In addition to the above options, occupancy sensors or motion sensors can help save energy by preventing TVs from being left on when people leave the room or fall asleep. Occupancy sensors become more important as it becomes easier to have TV displays in multiple rooms keyed to a primary source to enable users to not lose visual contact (or good audio) as they move from room to room.

Network connectivity and network standby are being widely discussed in the policy arena. Because of the complexity of network technologies and their associated protocols, and the rapid evolution of the connected TV technology and market, it is difficult to address network standby power in TVs independently and exhaustively in this report. To properly address this topic, it is important to understand other relevant topics, such as low-power mode policies in consumer electronics, including TVs, as well as digital network standards. For overall perspectives on network standby power, network technologies, and policy frameworks, we refer

\footnotetext{
73 It does not seem to represent all current smart TVs (connected TVs). According to CNET (2010), it takes about 45 seconds to power up Google TV normally.

${ }^{74}$ In digital electronics, a hardware register stores bits of information, in a way that all the bits can be written to or read out simultaneously. http://en.wikipedia.org/wiki/Hardware register
} 
the reader to the APP\&IEA 4E report (APP\&IEA 2010)and EuP Preparatory Studies Lot 6 and Lot 26 (Fraunhofer 2007(2), 2010). Even though more research is necessary, options exist to keep overall network standby power consumption low, and an important first step would be including network standby power in TV power-consumption test procedures. 


\section{Summary and Conclusions}

\subsection{TV Market and Technology Trends}

A] While TV sales and average TV screen sizes go up, inefficient displays are being replaced by efficient technologies.

Annual global TV shipments are expected to keep increasing, with a growth rate of 5.6\% from 2010 to 2014. Average screen area is also expected to increase, with a growth rate of $2.3 \%$ during the same period. LCD TVs, particularly LED backlit LCD TVs, are expected to dominate across the globe, accounting for more than $80 \%$ of the global TV market in 2014, and PDP TVs are expected to slowly decline. Although CRT TVs might remain popular in some emerging markets, major TV brands are likely to provide more affordable LCD TVs to replace CRT TVs. OLED TVs larger than 40 inches are expected to be realized in 2012, but they are not expected to become cost competitive with LCD TVs in the short term. Thus, LED backlit LCD TVs are expected to play an important role in TV energy savings. The increase in more efficient TV technologies on the market will somewhat offset the increases in energy consumption that would otherwise be expected from increasing sales and screen sizes.

B] TV production is globalized, however manufacturers will create additional new designs to accelerate LED-LCD TV penetration specifically in emerging markets.

TV manufacturing is highly globalized and concentrated; the top five manufacturers produce more than $60 \%$ of TVs sold worldwide, resulting in limited regional differences in screen technologies and sizes. However, major TV brands are expected to provide more LED backlit LCD TVs at lower prices in emerging markets. This new type of low-price model can be accomplished by decreasing the maximum luminance level and color-reproduction capability in LCD panels. Although we were not able to specifically forecast this trend in our analysis, these low-price models will consume less power than typical TVs in mature markets. It is uncertain whether there would be regional differences in the final assembly, e.g., power supply and tuner, of the same TV model from a given manufacturer.

C] Increasing 3D TV demand can directly and indirectly increase TV energy consumption, but development in screen technologies will reduce this negative effect.

3D TV demand is expected to rapidly increase, accounting for 32\% of the global TV shipment in 2014. An existing 3D TV in 3D mode is likely to consume more energy than in 2D mode because of 1) additional 3D image processing and 2) relatively lower brightness level, in which manufacturers might increase brightness level by consuming more power, than in 2D mode. However, manufacturers will overcome this increase in energy consumption by developing technologies and improving efficiency in both LCD panels and backlights. In addition, increasing demand for 3D TVs could be associated with an increase in average TV screen size on the market, which could also increase average energy consumption unless technological and efficiency improvements compensate for the increase. However, limited 3D content is currently available to consumers, so it is difficult to estimate average $3 \mathrm{D}$ usage.

D] Smart TVs (or Connected TVs) have the potential to consume more energy than conventional TVs.

Connected TVs (or Smart TVs) are expected to consume more energy compared to current non-connected TVs because of 1) advanced signal processing for network connectivity, 2) potential larger/wider screens, 3) increased daily usage, 4) default white background screens, and 5) network standby mode and fast start option. In particular, white backgrounds will negate or reduce the effect of dimming technology in LCD TVs. Network connectivity will cause these TVs to consume more power, $3 \mathrm{~W}$ to $30 \mathrm{~W}$, in network standby mode, compared to conventional passive standby power, which is less than $1 \mathrm{~W}$. 
E] Including network standby power in energy consumption test procedures would be an important first step toward addressing a possible increase in standby power consumption by connected TVs.

A connected TV (or a smart TV) basically performs a subset of what a PC does in sleep mode. PCs typically consume $2 \mathrm{~W}$ to $4 \mathrm{~W}$ of standby power. However, fast-start options may cause connected TVs to consume more than $25 \mathrm{~W}$ in standby mode, and the minimum power requirement for basic network processing varies among manufacturers. Current energy consumption test methods and regulations do not test TVs for consumption when connected to the network. However, if connected TV energy consumption in network standby mode were regulated, TV manufacturers would likely to improve their products so that standby consumption would be comparable to that of PCs.

F] For LCD TVs, learning rate, which determines the relationship between production cost (or price) and cumulative production, is expected to keep increasing over time in the near term, while the learning rate of PDP TVs is not.

Based on the TV market data from 2007 and 2010, the learning rate for LCD TVs is approximately 14.6\% and the rate for PDP TV is approximately $22.1 \%$. In other words, the learning rate of $14.6 \%$ indicates a $14.6 \%$ drop in price for a doubling in cumulative production of LCD TVs. The learning rate of $22.1 \%$ indicates a $22.1 \%$ drop in price for a doubling in cumulative production of PDP TVs. However, given that the global shipment of LCD TVs keeps growing while that of PDP TVs is expected to sustain the volume in the market, the learning rate of LCD TVs is expected to increase and that of PDP TVs is not. According to our analysis, the learning rate of LCD TVs is expected to increase from $14.6 \%$ to $18.5 \%$, and that of PDP TVs is expected to slightly decrease from $22.1 \%$ to $20.7 \%$, including the TV market forecast data from 2011 to 2014.

\subsection{Efficiency Improvement Potentials}

A] LCD TV efficiency will improve thanks to advanced backlight sources, efficient combinations of optical films, increased panel transmittance, and dynamic dimming methods.

\section{a. Backlight Sources: CCFL to LED, Simple Structure in LED backlights}

In general, LED backlight TVs are 20\% to 30\% more efficient than CCFL backlit TVs. A large-scale transition is expected from conventional CCFL backlit LCD TVs to LED backlit LCD TVs even in the absence of a market transformation program. In addition, LED-edge backlit LCD TVs are expected to dominate the market and to become more efficient by optimizing the backlight structure.

\section{b. LED Efficiency: High Efficacy LEDs}

LED efficacy will improve thanks to developments in advanced LED structure, phosphors, thermal management, and beam angles. Manufacturers can reduce material costs as a result of LED efficiency improvements, which provides an intrinsic motivation for manufacturers to achieve high efficiency in their LED backlights. However, the efficacy of LEDs used in TVs lags that of LEDs available on the lighting market because of technical issues, e.g., heat dissipation. If LED efficacy increases by $50 \%$ from $60 \mathrm{~lm} / \mathrm{W}$ to $90 \mathrm{~lm} / \mathrm{W}$, the effect on TV power will not be significant, e.g., only about a 15\% reduction in 32-inch LCD BLU power because LED power will likely increase as LED efficacy increases.

\section{c. Optical Films: Cost-effective Combinations}

Typical LCD backlights require prism film(s), diffuser(s), and a LGP or diffuser plate. A variety of combinations of optical films can go into the film stack, with varying performances and materials costs. DBEF is recognized as one of the best options for efficiency improvement in optical films, but it is a proprietary technology and seems expensive to manufacturers who want to minimize their production costs. As panel transmittance improves and new combinations of optical films are developed, it is anticipated that manufacturers will not need to use DBEF in the near future. BLU efficiency could nonetheless be improved by about $20 \%$ if DBEF were applied entry-level TV models. This analysis also considered a new LGP combined with prism film, which is expected to increase efficiency in LCD BLU 
by about $10 \%$. However, this option still faces technical challenges related to light distribution uniformity and picture quality.

\section{d. LCD Panel: High Panel Transmittance}

Improvement in LCD panel transmittance decreases the luminance that the backlight must achieve and therefore allows manufacturers to reduce the number of LEDs in the BLU. If panel transmittance improves by $50 \%$, from $5 \%$ to $7.5 \%$, total on-mode power is expected to decrease by about $20 \%$. The average transmittance of most LCD TV panels currently available on the market is between $5 \%$ and $6 \%$, and the highest panel transmittance is between $6 \%$ and $6.5 \%$. According to one manufacturer, if panel transmittance is improved from $6.5 \%$ to more than $8 \%$ and additional technologies are applied, the power consumed by a 32-inch HD LCD panel could decrease by more than 35\%, e.g., from $32 \mathrm{~W}$ to 20 W. In addition, higher panel transmittance allows manufacturers to design low-voltage-driven circuitry for LCD panels, which reduces overall TV power consumption. However, manufacturers can also save materials costs. The options for achieving the same luminance and image quality with better panel transmittance are: 1) to use less expensive (and less transmissive) optical films, or 2) to use fewer LEDs (and thereby reduce power consumption). Panel transmittance is expected to increase to up to $7 \%$ or $8 \%$ for a few manufacturers' products over the next few years, but this option requires R\&D investment rather than incremental costs. In addition, LCD panel technologies are proprietary and closely related to manufacturers' manufacturing processes.

\section{e. Power Management in On-Mode: Dynamic Dimming}

Because the effect of dimming methods varies with input images, dimming algorithm, and backlight structure, the average effect needs to be determined using the IEC 62087 standard video clip. The average effect is not analyzed in this report, but a $1 \mathrm{D}$ dimming method is expected to reduce BLU power by more than $15 \%$. Local dimming of LED-direct backlights will be more effective than partial dimming of LED-edge backlight in power reduction. Dynamic dimming in LCD TVs is a likely cost-effective option for improving efficiency because manufacturers employ $0 \mathrm{D}$ dimming or no dimming option for low-end products, and advanced dimming methods mostly for mid-range or/and high-end products because of incremental cost.

\section{f. Other Options: Color gamut, TN mode for Small Screen Size}

In general, the color gamut of an LCD TV is determined by color filter in the panel and phosphors in the backlight lamps. However, color gamut is traded off against efficiency, i.e., higher color gamut requires lower efficiency in materials. Recently, some TV manufactures have developed more efficient LED backlit LCD TVs at a lower manufacturing cost by reducing both the maximum luminance level and color gamut. In particular, there products are expected to target emerging markets. In addition, TN mode with high panel transmittance has been improved with the help of supplementary films, expanding its availability for slightly larger screen sizes (from 20-21 inches to 24-26 inches).

B] The average on-mode power of 32-inch LED-LCD TVs is expected to be improved by $20 \%$ from 2010 to 2012 as a result of improvement in LED efficacy, optical films, and panel transmittance. If a dimming method and DBEF are included, on-mode power will be further reduced by more than $10 \%$.

The average cost of DBEF for a 32-inch LCD TV was approximately $\$ 7$ to $\$ 8$ in 2010 . Assuming that the DBEF option can reduce the on-mode power of a 32-inch LED-LCD TV by $5 \mathrm{~W}$, the cost of conserved energy would be $\$ 0.08$ per $\mathrm{kWh}$ assuming a discount rate of $6 \%$ and an effective useful life of 10 years. In addition, it would cost an additional $\$ 3.2$ to $\$ 6.0$ to employ dimming options for 30 - to 50 -inch LCD TVs. Although the average effect of dimming options on reduction in power consumption was not determined in this report, it is expected to save more than $10 \%$ of on-mode power by both partial and local dimming options. Assuming that the dimming option can reduce the on-mode power of a 32-inch LED-LCD TV by 4 $\mathrm{W}$, the cost of conserved energy would range from $\$ 0.04$ per $\mathrm{kWh}$ to $\$ 0.08$ per $\mathrm{kWh}$ assuming a discount rate of $6 \%$ and an effective useful life of 10 years. As DBEF and dimming options are mature technology and being improved by manufacturers, these would be good candidates for cost-effective options in LCD TV 
efficiency improvement.

C] Most LCD efficiency improvement options can be applied to all screen sizes.

Most LCD efficiency improvement options are applicable to all screen sizes, but technologies to improve panel transmittance are limited to specific LC structures such as TN, VA, and IPS. Major manufacturers have developed their own proprietary technologies. Although TN mode is available only for small screen sizes (smaller than 30 inches), TN's transmittance is better than that of other LC modes. In addition, the effect of dimming methods is limited to structure of backlights. While LED-edge backlights are capable of using only partial dimming methods, LED-direct backlights can use all types of dimming methods.

D] PDP TV efficiency can be improved by high-efficiency panels and improvements in reactive power and filter transmittance. High-efficiency panel technologies play an important role in improving overall PDP performance, manufacturing process, and total cost.

PDP efficiency and overall performance are expected to be improved by design of improved cell structures, phosphors, new electron emission layers, and a low-voltage driving scheme. These technological options are interconnected and difficult to isolate from one another to determine their individual effect on TV on-mode power consumption. Most technologies related to panel efficiency improvements require R\&D investment rather than simply incremental material costs.

E] The on-mode power of an existing 30- to 32-inch (76.2- to 81.3-cm) OLED TV (prototype) is estimated to be about $30 \mathrm{~W}$ to $40 \mathrm{~W}$. There are potential efficiency improvements for OLEDs.

OLED materials account for $60 \%$ to $70 \%$ of the power consumed by the OLED panel; the rest is for the TFT drive. Phosphorescent materials are being improved and are expected to replace fluorescent materials. In addition, improvement in TFT mobility will increase TFT drive efficiency. Potential OLED efficiency improvement options are strongly related to proprietary OLED technologies and manufacturing processes.

F] Based on our identification of efficiency improvement options and data for ENERGY STAR Version 4 qualified TVs, all TV technologies ( $\leq 50$ inches) are expected to be able to meet or approach ENERGY STAR Version 5 levels in 2012.

The average on-mode power for 42-inch LED backlit LCD TVs is expected to be about $20 \%$ below ENERGY STAR Version 5. The average for 42 -inch OLED TVs is expected to be about $30 \%$ to $40 \%$ below ENERGY STAR Version 5. However, this forecast is based on on-mode power in default home mode. Current TVs still consume more or less power in modes other than the default home mode.

G] Although most new panel-related technologies require R\&D investment rather than incremental cost, they can improve manufacturing processes and save costs.

In general, each TV panel manufacturer has its own proprietary technology for FPDs, e.g., LCD, PDP, and OLED. Technology options related to cell structure and materials are complex and interconnected. However, improvement in panel efficiency can allow manufacturers to use low-voltage or low-current circuitry and to reduce the number of other panel components. Sometimes, panel efficiency improvements can allow manufacturers to eliminate manufacturing steps, resulting in lower production costs. Most panel-related technologies are classified as Type (A) in Figure 7-1. Optical components, e.g., films and filters, and electrical components would be more appropriate cost-effective options, categorized as Type (B) in Figure 7-1, than panel technologies. 


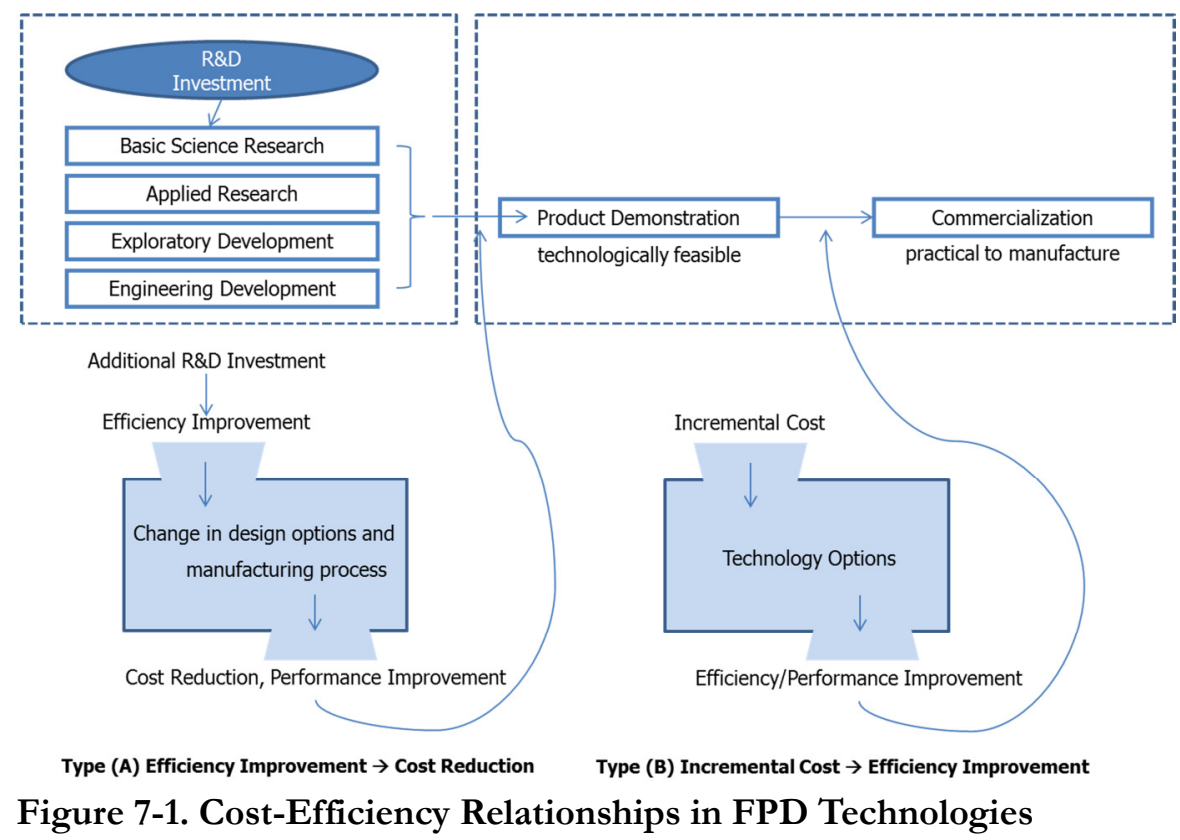

$\mathrm{H}] \mathrm{ABC}$ settings seem essential for large screen size (>40") TVs while small screen size (<30") TVs still have potential to reduce on-mode power if $\mathrm{ABC}$ is incorporated in the default mode.

More than $80 \%$ of ENERGY STAR-qualified TVs over 40 inches had ABC enabled when they were shipped, and about 30\% of ENERGY STAR-qualified TVs below 40 inches had the same conditions. Therefore, for smaller-screen TVs, there are still savings to be realized by enabling ABC when units are shipped.

I] The effect of current $\mathrm{ABC}$ settings on average on-mode power consumption ranges from $5 \%$ to $30 \%$, depending on screen or backlight technology and screen size. Although ABC is encouraged by default for all TVs, energy consumption test methods to account for $\mathrm{ABC}$ need to be revised to test at more realistic ambient lighting levels.

The magnitude of the effect of $\mathrm{ABC}$ on power consumption is significant, but current test procedures that allow for crediting estimated energy savings to TVs that have ABC can provide counterproductive incentives to manufacturers to set $\mathrm{ABC}$ so that the picture at measurement is too dark. A recent draft ENERGY STAR Version 6 (ENERGY STAR 2011c) includes an updated test method for ABC. On-mode power consumption for TVs with $\mathrm{ABC}$ is expected to be calculated as a weighted average of power consumption at various ambient lighting levels.

\subsection{Energy Consumption and Savings Potentials}

A] TVs in on-mode are estimated to consume more than about $168 \mathrm{TWh}$ in 2010, representing $3 \%$ to $4 \%$ of global residential electricity. The consumption is expected to be similar or slightly decrease in the short term because of technological transition (CRT to LCD, and CCFL-LCD to LED-LCD) and rapid improvements in TV energy efficiency, in spite of the projected increase in TV sales and average screen size.

B] Annual electricity savings potential for the efficient case with cost-effective options in this analysis is about 3.2 TWh in 2015 and 13.6 TWh in 2030. Cumulative electricity savings potential is about 6.4 TWh from 2012 through 2015 and 156 TWh through 2030.

In this scenario, only $30 \%$ of LCD TVs were assumed to be efficient. Estimated minimum effects of cost- 
effective options (DBEF and 1D dimming option) were applied.

C] About $1.4 \mathrm{Mt} \mathrm{CO}$ in 2015 and about 6.2 $\mathrm{Mt} \mathrm{CO}_{2}$ in 2030 can be saved annually in the efficiency case. Cumulative electricity savings potential is about $3.1 \mathrm{Mt} \mathrm{CO}_{2}$ from 2012 through 2015 and 70.9 Mt $\mathrm{CO}_{2}$ through 2030.

D] It is roughly estimated that the additional energy consumption for 3D mode of 3D TVs in 2014 will be about $0.2-1.2$ TWh in the absence of any energy-efficiency improvement.

In this scenario, an additional $20 \mathrm{~W}$ was assumed to be consumed for 3D mode of a TV. 2 hours per day were assumed to be spent for 3D content by the viewer. While average daily hours spent by viewers are uncertain, it is expected that 3D technologies and screen efficiency will improve over time.

E] It is roughly estimated that fast start modes can consume from about 4 TWh to 19 TWh in 2014, assuming that $10-50 \%$ of LCD TVs ( $\geq 30$ inches) will be operated in the modes, consuming $25 \mathrm{~W}$.

Although fast start mode is not a necessarily network-related function, the feature usually coincides with network connectivity in increasing connected TVs. It is also necessary to consider both features together.

F] It is roughly estimated that emerging connected TVs (or smart TVs) are likely to consume from about 3 TWh to about 27 TWh in 2014, based on the projected increase in connected TV sales and average network standby power, ranging from $3 \mathrm{~W}$ to $30 \mathrm{~W}$.

Although energy savings potential in TV network standby mode is not included in the BUENAS modeling, the annual energy consumption by new connected TV sales in 2014 is estimated to be from 2.7 to 26.7 TWh, and the cumulative energy consumption by connected TV sales from 2010 through 2014 is expected to be from 9.3 to $92.6 \mathrm{TWh}$, depending on power management regime and fast start option applied.

\begin{tabular}{l|l|r}
\hline \multicolumn{2}{c|}{ Modes } & \multicolumn{1}{c}{ Power consumption } \\
\hline \multirow{3}{*}{ On } & Normal & $\sim 70-80 \mathrm{~W}(45-100 \mathrm{~W})$ \\
\cline { 2 - 3 } & 3D mode & *40"-42" LED-LCD TVs (ENERGY STAR) \\
\hline \multirow{3}{*}{ Standby } & Network & $3-30 \mathrm{~W}$ \\
\cline { 2 - 3 } & Fast Start & $\sim 25 \mathrm{~W}(11-68 \mathrm{~W})$ \\
\cline { 2 - 3 } & Data Acquisition & $\sim 20 \mathrm{~W}(10-40 \mathrm{~W})$ \\
\hline
\end{tabular}

Table 7-1 Summary Table of Power Consumption in Various Modes of TVs 


\begin{tabular}{l|l|l|l|r|r}
\hline \multicolumn{2}{l|}{ Modes } & $\begin{array}{l}\text { Contribution } \\
\text { from }\end{array}$ & 2010 & 2014 & $\begin{array}{l}\text { Cumulative consumption } \\
\text { from 2010 through 2014 }\end{array}$ \\
\hline \multirow{3}{*}{ On } & Normal & global stock & $168 \mathrm{TWh}$ & $171 \mathrm{TWh}$ & $857 \mathrm{TWh}$ \\
\cline { 2 - 6 } & 3D mode & global sales & $0.02-0.04 \mathrm{TWh}$ & $0.2-1.2 \mathrm{TWh}$ & $0.8-3.0 \mathrm{TWh}$ \\
\hline \multirow{3}{*}{ Standby } & Network & global sales & $1.0-9.6 \mathrm{TWh}$ & $2.7-26.9 \mathrm{TWh}$ & $9.3-92.6 \mathrm{TWh}$ \\
\cline { 2 - 6 } & Fast Start & global sales & $2.6-7.9 \mathrm{TWh}$ & $3.9-19.3 \mathrm{TWh}$ & $16.6-71.5 \mathrm{TWh}$ \\
\cline { 2 - 6 } & Data Acquisition & global sales & $0.2-0.7 \mathrm{TWh}$ & $0.3-1.0 \mathrm{TWh}$ & $1.5-4.4 \mathrm{TWh}$ \\
\hline
\end{tabular}

a: 3D TV market forecast, b: Connected TV market forecast, c: 10-30\% (2010) and 10-50\% (2014) of LCD TV shipment ( $\geq 30$ inches), d: $10-30 \%$ of LCD and PDP TV shipment ( $\geq 30$ inches)

Table 7-2 Summary Table of Energy Consumption Forecast in TVs 


\section{Acknowledgments}

Michael McNeil and Jing Ke of Lawrence Berkeley National Laboratory provided support for the BUENAS modeling effort. Nan Wishner of Lawrence Berkeley National Laboratory provided editing support. Frank Klinckenberg, Steve Pantano,Ana Maria Carreño, and Elisa Lai of the Collaborative Labeling and Appliance Standards Program(CLASP) coordinated the peer review process by experts and stakeholders. Helpful comments were provided on this report by Mia Forbes Pirie and Louis Benoit- Desroches of Lawrence Berkeley National Laboratory's Appliance Standards Group from the United States(US), Bruce Nordman of Lawrence Berkeley National Laboratory's Energy End-Use Forecasting Group from the United States(US), Chris Calwell of ECOS Consulting from the United States(US), Margie Lynch and Seth Wylie of the Consortium for Energy Efficiency(CEE), Noah Horowitz of the Natural Resources Defense Council (NRDC) from the United States(US), Robert E. Kopp of U.S. Department of Energy, Steve Pantano of Collaborative Labeling and Appliance Standards Program (CLASP) from the United States (US), Bob Harrison of Intertek UK from the United Kingdom(UK), Keith Jones of Digital Cenergy from Australia, Jeremy Tait of Tait Consulting and IEA 4E Mapping \& Benchmarking operating agent, from the United Kingdom (UK), Geoff Bellingham from the United Kingdom(UK), Alain Anglade of Agence de l'Environnement et de la Maitrise de l'Energie (ADEME) from France, and Mikael Holst of the Swedish Energy Agency from Sweden. Any errors or omissions are the author's own.

\section{References}

Asia Pacific Partnership (APP) and International Energy Agency (IEA) 4E standby Annex. 2010. "Standby Power and Low Energy Networks - issues and directions", September. http://standby.iea-4e.org/files/otherfiles/0000/0023/Network-Standby-2010-09-final.pdf

Baker, Simon, TFT Central. 2011. "Panel Technologies - TN Film, MVA, PVA and IPA Explained". March. http://www.tftcentral.co.uk/articles/panel technologies.htm

Barker, D. 2006. "Pioneer's Crystal Emissive Layer for plasma TV: How it works." EE Times. July 26. http://www.eetimes.com/design/other/4013071/Pioneer-s-Crystal-Emissive-Layer-for-plasma-TV-How-it -works.

Bogner, T. 2010. “Top Ten Product Criteria Paper on Television and television sets.” Austrian Energy Agency, June.

Chen, H.F. 2006. “Backlight Local Dimming Algorithm for High Contrast LCD-TV.” Samsung Electronics, Society for Information Display.

Chinnock, C. 2011. “The Slippery Slope of 3D Brightness”, Consumer Lifestyle News. May. http: $/ /$ www.cln-online.org/index.php?option $=$ com content\&view $=$ article\&id $=1784$ :understanding- $3 \mathrm{~d}$ tv\&catid=65:all-about-3d-tv

Choi, S.J. 2010. “3D TV Technology Trend and Forecast” LG Electronics, October. http://www.itconference.co.kr/141779

Chung, Y.S. 2010. “3D Technology and its Display Trends.” DisplaySearch, 2010 China FPD Conference.

CNET. 2010. Sony NSX-GT1 series: Quick Start mode. November. http://reviews.cnet.com/2300-6482 7-10005442-27.html?s $=0 \& \mathrm{o}=10005442$

DisplaySearch. 2009. "India TV Data." August.

DisplaySearch. 2010a. "Brazil TV Data.” January.

DisplaySearch. 2010b. “Quarterly Advanced Global TV Shipment and Forecast Report, First Quarter, 2010”, April.

DisplaySearch. 2010c. "Quarterly Advanced Global TV Shipment and Forecast Report, Third Quarter, 2010", October. 
DisplaySearch. 2010d. “Quarterly Large-area LCD Cost Model, Third Quarter, 2010”, October.

DisplaySearch. 2010e. "Quarterly TV Cost and Price Forecast Model \& Report, First Quarter, 2010”

DisplaySearch. 2010f. "Quarterly TV Cost and Price Forecast Model \& Report, Third Quarter, 2010", October.

DisplaySearch. 2010g. "Quarterly TV Cost and Price Forecast Model \& Report, Fourth Quarter, 2010”, December.

DisplaySearch. 2011a. "Quarterly Advanced Global TV Shipment and Forecast Report, Fourth Quarter, 2010", January.

DisplaySearch. 2011b. “LED\&CCFL Backlight Cost Report, Fourth Quarter, 2010”, January.

DisplaySearch. 2011c. “Optical Film Report. Fourth Quarter, 2010”, February.

DisplaySearch. 2011d. “3D TV Forecast Data”, February.

DisplaySearch. 2011e. "Connected TV Forecast Data”, February.

DisplaySearch. 2011f. “Quarterly Advanced Global TV Shipment and Forecast Report, First Quarter, 2011.” March.

DisplaySearch. 2011g. "Quarterly TV Cost and Price Forecast Model \& Report, First Quarter, 2011”, April.

Ecos. 2010. “Assessment of Options for Improving Energy Efficiency Test Procedures for Displays”, March.

Energy Conservation Center, Japan (ECCJ). 2009. "2008 Standby Power Consumption Report”, March. http://www.eccj.or.jp/standby/08/standby power product report 2008.pdf

ENERGY STAR. 2007. "ENERGY STAR Qualified Televisions Specification Revision Update”, January http://www.energystar.gov/ia/partners/prod development/revisions/downloads/tv vcr/TV update doc ument Final.pdf

ENERGY STAR. 2010. “ENERGY STAR Qualified TV List, 2010”, November.

ENERGY STAR. 2011a. “ENERGY STAR Qualified TV List, 2011”, March.

ENERGY STAR. 2011b. "ENERGY STAR Program Requirements Product Specification for Televisions: Eligibility Criteria Version 5.3"

http://www.energystar.gov/ia/partners/prod development/revisions/downloads/television/V5.3 Progra $\underline{\text { m Requirements.pdf }}$

ENERGY STAR. 2011c. "ENERGY STAR Program Requirements Product specification for Televisions: Eligibility Criteria Draft 1 Version 6.0" http://www.energystar.gov/ia/partners/prod development/revisions/downloads/television/D1_V6.0_Sp ec.pdf

ENERGY STAR. 2011d. “ENERGY STAR Desktop \& Integrated Computer Product List, 2011”, May.

European Commission. 2009. EC Full Impact Assessment of Eco Design Measure. July. http://ec.europa.eu/energy/efficiency/ecodesign/doc/legislation/sec 2009 1011.pdf

Fraunhofer IZM. 2007. "EuP Preparatory Studies “Televisions” (Lot 5)”, August. http://www.ecotelevision.org/finalised documents.php

2007a. Final Report on Task 1 "Definition"

2007b. Final Report on Task 3 "Consumer Behaviour and Local Infra Structure"

2007c. Final Report on Task 4 "Technical Analysis"

2007d. Final Report on Task 6 "Technical Analysis BAT"

2007e. Final Report on Task 7 "Improvement Potential"

2007f. Final Report on Task 8 "Scenario, Policy, Impact and Sensitivity Analysis" 
Fraunhofer Institute for Reliability and Microintegration, IZM. 2007(2). "EuP Preparatory Studies Lot 6: Standby and Off-mode Losses", October.

2007(2)a. Final Report Task 1 Definition

2007(2)b. Final Report Task 6 Technical Analysis BAT

Fraunhofer IZM. 2010. "EuP Preparatory Studies Lot 26: Network Standby Losses"

http://www.ecostandby.org/documents.php

2010a. Draft Final Report Task 1 Definition

2010b. Draft Final Report Task 4 Technical Analysis Existing Products

2010c. Draft Final Report Task 5 Definition of Base Cases

2010d. Draft Final Report Task 6 Technical Analysis BAT

Halsted, C.P. 1993 “Brightness, Luminance, and Confusion”. Society for Information Display (SID).

Hanzawa, R. 2009. "Lens Films and Reflective Polarization Films.” Sumitomo 3M.

http://media.wiley.com/product data/excerpt/71/04706996/0470699671-8.pdf

Hsieh, H. H., et al. 2010. “Next Generation TFT Technology for AMOLED.” AU Optronics Corp., IMID.

ICF International. 2011. ENERGY STAR Qualified TVs with ABC-disabled, March.

IEC 62087, 2011. International Standard- Method of measurement for the power consumption of audio, video and related equipment, Edition 3. Draft for IEC 62087/Ed.3/CDV. International Electrotechnical Commission. Geneva.

IEC 62301. 2011. International Standard: Household electrical appliances - Measurement of standby power, 2011-01.

International Energy Agency (IEA). 2006. "World Energy Outlook".

International Energy Agency (IEA), Efficient Electrical End-Use Equipment (4E). 2009. "Product Definition: Televisions". July.

International Energy Agency (IEA), Efficient Electrical End-Use Equipment (4E). 2010a. "Benchmarking Energy Efficiency of New Televisions". October. http://mappingandbenchmarking.iea-4e.org/shared files/110/download

2010b Australia

2010c EU

2010c France

2010d Republic of Korea

2010e USA

International Energy Agency (IEA). 2008-2010. Key World Energy Statistics.

International Standby Basket of Products. The Australian Government's the Department of Climate Change and Energy Efficiency, 2007-2010. http://www.energyrating.gov.au/standbydata/app/Data.aspx

IP Network. 2010. Television International Key Facts 2010. http://www.ipnetwork.com/comm files/IPNTVKF2010/Key\%20Table \%20\%20Viewing $\% 20$ Time $\% 20$ per $\% 20$ Individual.pdf

Jo et al. 2009. "Optimizing the Startup Time of Embedded Systems: A Case Study of Digital TV”. IEEE Transactions on Consumer Electronics, vol 55, No 4, November.

Kang et al. 2010. "Recent Progress of LG PDP: High Efficiency \& Productivity Technologies." LGEARI, IMID.

Khorami et al. 2010. "Effect of Protective Layers on the Cell efficiency of Plasma Display Panels." United 
Kingdom: University of Essex, IMID.

Kim et al. 2009. "Emerging Technologies for the Commercialization of AMOLED TVs." Samsung Mobile Display, Society for Information Display (SID).

Kim, Y. 2010 “Negative Label, Positive Step: Standby Warning Label and Korea's 1W Policy". Presentation at Asia-Pacific Economic Cooperation (APEC) International Conference: Alignment of Standby Power Approaches Moving towards 1 Watt and Beyond. Korea Energy Management Corporation, October. http://www.energyrating.gov.au/standby2010-apec-presentations.html

Kwon et al. 2010. "LED Backlighting Driving Circuits and Dimming Method.” Hanyang University, IMID.

Lee, S.B. 2008. "Image Quality Technology Issues using Emotional Image Quality Evaluation Method", Samsung SDI.

Letschert, V., M. A. McNeil, N. Zhou. "Residential Electricity Demand in China -Can Efficiency Reverse the Growth". EEDAL 2009. LBNL 2321E.

Market Transformation Programme. 2010. "BNCE04: Televisions (TVs) Government Standards Evidence Base 2009: Best Available Technology Scenario.” version 1.0. February.

McNeil, M. A., V. E. Letschert, and S. de la Rue du Can. 2008. "Global Potential of Energy Efficiency Standards and Labeling Programs.” Lawrence Berkeley National Laboratory. November. http://ies.lbl.gov/iespubs/LBNL-760E.pdf

Ministry of Knowledge Economy (MKE), Korea. 2010. "Smart TV Leadership and the Market Forecast", Monthly Display. November.

Natural Resources Defense Council/Ecos Consulting. 2005. "Televisions: Active mode Energy Use and Opportunities for Energy Savings." March.

Nordman et al. 2009. "Network Connectivity and Low Power Mode Energy Consumption", Energy Efficiency in Domestic Appliances and Lighting (EEDAL), June.

Nordman, B. and S. Lanzisera. 2011. "Inter-device power management for audio/video equipment", presented at 2011 International Conference on Consumer Electronics, January. http://eetd.lbl.gov/ea/nordman/

Nielsen. 2009. A2/M2 Three Screen Report, $1^{\text {St }}$ quarter 2009. http://blog.nielsen.com/nielsenwire/wp-content/uploads/2009/05/nielsen threescreenreport q109.pdf

OECD. 2009. "OECD Communications Outlook." http://www.oecd.org/document/44/0,3746,en_2649_34225_434353008_1_1_1_1,00.html

OLED Association. 2010. "Continued Advances in OLED Technologies and Materials Accelerate the Emergence of New High Performance Displays and Lighting Products" http://www.oled-a.org/images/pdfs/Lighting\%20White\%20Paper\%2010-1.pdf

Otani, T. 2008. “Samsung Exhibits 63-inch 4k x 2k PDP.” Tech-On. October 30. http://techon.nikkeibp.co.jp/english/NEWS_EN/20081030/160443/

Phadke, A. and J. Sathaye. 2010. "SEAD Incentive Coordination for Televisions: An Illustrative Analysis." preliminary technical notes. Lawrence Berkeley National Laboratory. July.

Price et al. 2006. "Sectoral Trends in Global Energy Use and Greenhouse Gas Emissions". LBNL-56144 http://ies.lbl.gov/iespubs/56144.pdf

Jang, T. 2009. "LED backlight Technology for LED TV”. Crystal Valley Conference \& Exhibition, Korea. Presentation. Samsung Electronics. November.

Samsung Mobile Display. 2009. "Emerging Technologies for the Commercialization of AMOLED TVs." Society for Information Display.

Sathaye, J. and A. Phadke. 2010. "Energy Efficiency Cost Curves: Empirical Insights for Energy-Climate modeling". Modeling the Economics of Greenhouse Gas Mitigation: Summary of a Workshop, National 
Research Council. The National Academies Press. ISBN-10: 0-309-16235-1. p52-68.

Tseng, F., Cheng, A. 2009. "Combining Scenario Analysis with Delphi and the Technological Substitution Model to Analyze the Development of the OLED TV Market". August.

TGI. 2009. TGI Update 6: Media Usage. http://globaltgi.com/knowledgehub/documents/TGIUpdate3.pdf

3M LCD Optics 101. 2010a. http://solutions.3m.com/wps/portal/3M/en US/Vikuiti1/BrandProducts/secondary/optics101/

3M. 2010b. Flat Panel Display (FPD) International Exhibition/Conference, Japan.

Toshiba. 2011. "Toshiba Enhances Accessibility with Multiple 3D Technologies." http://us.toshiba.com/pressrelease/509560?fromPage=editorials

United Kingdom Department for Environmental Food \& Rural Affairs. 2009. "Impact Assessment of implementing Measures for Ecodesign Requirements and Energy Labelling of Televisions (TVs)." March. http://www.ialibrary.berr.gov.uk/ImpactAssessment/?IAID=f8a207eb43924213be9bdb1dca17b732

United States Department of Energy (U.S. DOE). 2009. "Preliminary Technical Support Document: Energy Efficiency Program for Consumer Products.” November.

United States Department of Energy (U.S. DOE). 2010a. "Solid-State Lighting: Brilliant Solutions for America's Energy Future.” June.

United States Department of Energy (U.S. DOE). 2010b. "Solid-State Lighting Research and Development: Multi-Year Program Plan.” March. United States Department of Energy (U.S. DOE). 2011. "Using the experience Curve Approach for Appliance Price forecasting", February 2011.

http://www1.eere.energy.gov/buildings/appliance standards/pdfs/experience curve appliance price for ecasting 3-16-11.pdf

United States Department of Energy (U.S. DOE). 2010c. "U.S. DOE International Energy Outlook 2010" http://www.eia.gov/oiaf/ieo/images/xls.gif

U.S. Department of Labor, Bureau of Labor Statistics. 2011. Consumer Price Index, All Urban Consumers (CPI-U), U.S. city Average, All items, April 15. ftp://ftp.bls.gov/pub/special.requests/cpi/cpiai.txt

Wang, Jing. 2008. "Brand New China: Advertising, Media, and Commercial Culture”. p248.

Waide, P., Navigant Consulting. 2011. "Opportunities for Success and CO2 savings from Appliance Energy Efficiency Harmonisation”. March. http://www.clasponline.org/clasp.online.resource.php?disdoc=781

Weber, L.F. 2005. “The Race for TVs with Higher Luminous Efficiency,” IMID.

Whang, K.W. 2010. "Address Discharge Characteristics of High Luminous Efficacy AC PDP with SrO-MgO Double Protecting Layer”. Seoul National University. IMID.

Wikipedia. 2011a. Smart TV. June 20, 2011 http://en.wikipedia.org/wiki/Smart TV

Wikipedia. 2011b. Luminance, Luminous flux, Luminous intensity, and Solid angle. June 20, 2011 http://en.wikipedia.org/wiki/Luminous flux http://en.wikipedia.org/wiki/Luminous intensity http://en.wikipedia.org/wiki/Luminance http://en.wikipedia.org/wiki/Solid angle

Young, B. 2009. "OLEDs - Promises, Myths, and TVs," Society for Information Display.

Young, R., YS Chung. 2007. “The Future Still Looks Bright for Plasma TVs.” DisplaySearch. 


\section{Appendix A. SEAD Countries}

Countries Included in DisplaySearch Global TV Shipment and Forecast Report

\begin{tabular}{|c|c|}
\hline Region & Countries Included \\
\hline Japan & Japan \\
\hline North America & $\underline{\text { Canada and USA }}$ \\
\hline Western Europe & $\begin{array}{l}\text { Andorra, Austria, Belgium, Denmark, Finland, France, Germany, United Kingdom, } \\
\text { Greece, Greenland, Iceland, Italy, Liechtenstein, Luxembourg, Malta, Monaco, } \\
\text { Netherlands, Norway, Portugal, San Marino, Spain, Sweden, and Switzerland }\end{array}$ \\
\hline Eastern Europe & $\begin{array}{l}\text { Albania, Belarus, Bosnia and Herzegovina, Bulgaria, Croatia, Cyprus, Czech } \\
\text { Republic, Estonia, Hungary, Latvia, Lithuania, Moldova, Poland, Romania, Russia, } \\
\text { Serbia and Montenegro, Slovakia, Slovenia, Turkey and Ukraine }\end{array}$ \\
\hline China & Includes Hong Kong, but for Taiwan \\
\hline Asia Pacific & $\begin{array}{l}\text { Afghanistan, Australia, Bangladesh, Bhutan, Brunei, Burma (Myanmar), Cambodia, } \\
\text { Cook Islands, East Timor, Fiji, French Polynesia, India, Indonesia, Kiribati, Laos, } \\
\text { Malaysia, Maldives, Marshall Islands, Micronesia, Mongolia, Nauru, Nepal, New } \\
\text { Zealand, New Caledonia, Niue, North Korea, Pakistan, Palau, Papua New Guinea, } \\
\text { Philippines, Samoa, Singapore, Solomon Islands, South Korea, Sri Lanka, Taiwan, } \\
\text { Thailand, Tonga, Tuvalu, Vanuatu, and Vietnam }\end{array}$ \\
\hline Latin America & $\begin{array}{l}\text { Antigua, Argentina, Aruba, Bahamas, Barbados, Belize, Bolivia, Bonaire, Brazil, } \\
\text { Cayman Islands, Chile, Columbia, Costa Rica, Cuba, Curacao, Dominica, Dominican } \\
\text { Republic, Ecuador, El Salvador, French Guiana, Grenada, Guatemala, Guyana, } \\
\text { Haiti, Honduras, Jamaica, Martinique, Mexico, Nicaragua, Panama, Paraguay, Peru, } \\
\text { Puerto Rico, St. Kitts/Nevis, St. Lucia, St. Vincent and the Grenadines, Suriname, } \\
\text { Trinidad and Tobago, Turks \& Calicos Islands, Uruguay, Venezuela and the Virgin } \\
\text { Islands }\end{array}$ \\
\hline $\begin{array}{l}\text { Middle East and } \\
\text { Africa }\end{array}$ & $\begin{array}{l}\text { Algeria, Angola, Bahrain, Benin, Botswana, Burkina Faso, Burundi, Cameroon, Cape } \\
\text { Verde, Central African Republic, Chad, Comoros, Congo, Cote d'Ivoire, Djibouti, } \\
\text { Egypt, Equatorial Guinea, Eritrea, Ethiopia, Gabon, Gambia, Ghana, Guinea, Iraq, } \\
\text { Israel, Jordan, Kenya, Kuwait, Lebanon, Lesotho, Liberia, Libya, Madagascar, } \\
\text { Malawi, Mali, Mauritania, Mauritius, Morocco, Mozambique, Namibia, Niger, } \\
\text { Nigeria, Oman, Palestine, Qatar, Reunion, Rwanda, Sao Tome \& Principe, Saudi } \\
\text { Arabia, Senegal, Seychelles, Sierra Leone, Somalia, South Africa, Sudan, Swaziland, } \\
\text { Syria, Tanzania, Togo, Tunisia, UAE, Uganda, Western Sahara, Yemen, Zambia and } \\
\text { Zimbabwe }\end{array}$ \\
\hline
\end{tabular}

As of April 2011, the governments participating in SEAD are: Australia, Brazil, Canada, the European Commission, France, Germany, India, Japan, Korea, Mexico, Russia, South Africa, Sweden, the United Kingdom and the United States. (Underlined) This report is analyzing the SEAD members, except for UAE, as of CEM-1. 


\section{Appendix B. Data, Methodology and Assumptions}

The data and information included in this analysis are based on various sources. Some of the data required adjustment or generalization with corresponding simplifying assumptions. To the extent possible, variability in quality of data and uncertainty of forecasts have been taken into account during our analysis.

\section{B1. TV Market Data}

The TV market data is based on the DisplaySearch report; Quarterly Advanced Global TV Shipment and Forecast Report (2010 Q1 - 2011 Q1). The report provides various TV market analysis viewpoints by technology, region, brand, and backlight type. The forecast extends to the year 2014 and provides data based on eight regions: Asia Pacific (AP), China, Japan, Eastern Europe (EE), Western Europe (WE), Latin America (LA), North America (NA), and Middle East \& Africa (ME\&A). Data are not provided on a country-specific basis except for China and Japan. Data for India are provided separately by DisplaySearch.

To estimate TV shipment data for SEAD-14, this report assumes country-specific factors suggested by discussions with marketing experts from the industry and DisplaySearch. The assumed factors are based on LCD TV sales for recent years in each country. The forecast for country-specific TV sales from 2010 to 2014 follows the regional growth rates from DisplaySearch's analysis. For example, the LCD share of the U.S. market is assumed to be the same as the LCD share of the North America regional market.

\section{TV Shipments or Sales}

Australia : (sales) $20 \%$ of Asia Pacific shipment

Canada : (shipments) $11 \%$ of North America shipment

China : (shipments) DisplaySearch (2011a)

France : (sales) $20 \%$ of Western Europe shipment

Germany : (sales) 23\% of Western Europe shipment

India : (shipments) DisplaySearch (2009)

Japan : (shipments) DisplaySearch (2011a)

Korea : (sales) $16 \%$ of Asia Pacific shipment

Mexico : (sales) $24 \%$ of Latin America shipment

Russia : (sales) $45 \%$ of Eastern Europe shipment

South Africa : (sales) $9 \%$ of Middle East and Africa shipment

Sweden : (sales) 3\% of Western Europe shipment

UK : (sales) $25 \%$ of Western Europe shipment

US : (shipments) $89 \%$ of North America shipment

Assumption: Growth rates of LCD, PDP, CRT, and OLED TVs for 9 countries from 2010 to 2014 are the same as those of the corresponding regions. 


\section{B2. TV Efficiency Improvement Options}

\section{A] On-mode Power Data}

Data for on-mode power consumption used in the analysis are from the U.S. ENERGY STAR TV list posted on November 1, 2010 and March 22, 2011. Although the ENERGY STAR list includes only ENERGY STAR-qualified products, market penetration of ENERGY STAR-qualified TVs was more than $60 \%$ in $2006,{ }^{75} 79 \%$ in $2008^{76}$, and, in $2010,70 \%$ of all TVs in the U.S. carried the ENERGY STAR $\log { }^{77}$. The ENERGY STAR TV list for this study includes more than $700 \mathrm{TV}$ models and their on-mode power consumption but does not break down LCD TVs by backlight type. Therefore, we sorted LED backlit TVs from the list according to manufacturer catalogs and other data.

We assume that the ENERGY STAR data represent the U.S. market, but these data may not be representative for other countries. Instead, we reflected screen technology mixtures, including LCD backlight types, for each region. We also used Energy Consumption Center, Japan (ECCJ) efficient TV data. ${ }^{78}$ Although these data do not specify on-mode power consumption, on-mode power can be back calculated from annual energy consumption, standby power, and assumed viewing hours. The IEC 62087 standard video clip used to measure on-mode power for U.S. products does not measure on-mode power for Japanese products ${ }^{79}$ so we separately used the ECCJ data to compare rated power and on-mode power of the products in the ECCJ data set. Although these two data sets represent only efficient TVs in the U.S. and Japan, it is assumed that models available in these two markets are representative of a large majority of markets.

\begin{tabular}{|c|c|c|c|c|c|c|c|c|}
\hline \multirow[b]{2}{*}{ Source } & \multirow[b]{2}{*}{ Year } & \multicolumn{4}{|c|}{ Number of Models included } & \multirow{2}{*}{$\begin{array}{l}\text { On-mode } \\
\text { power }\end{array}$} & \multirow{2}{*}{$\begin{array}{c}\text { Standby } \\
\text { (or Sleep) } \\
\text { Power } \\
\end{array}$} & \multirow{2}{*}{$\begin{array}{l}\text { Rated } \\
\text { power }\end{array}$} \\
\hline & & Total & LCD & PDP & CRT & & & \\
\hline $\begin{array}{l}\text { U.S. ENERGY } \\
\text { STAR }\end{array}$ & $\begin{array}{l}2010 \\
\text { (Oct) }\end{array}$ & 731 & 644 & 65 & 0 & available & available & $\mathrm{N} / \mathrm{A}$ \\
\hline $\begin{array}{l}\text { U.S. ENERGY } \\
\text { STAR }\end{array}$ & $\begin{array}{l}2011 \\
\text { (Mar) }\end{array}$ & 1,029 & 910 & 119 & 0 & available & available & $\mathrm{N} / \mathrm{A}$ \\
\hline $\begin{array}{l}\text { U.S. ENERGY } \\
\text { STAR (ABC } \\
\text { disabled) }\end{array}$ & $\begin{array}{l}2011 \\
\text { (Mar) }\end{array}$ & 889 & 807 & 82 & 0 & available & available & $\mathrm{N} / \mathrm{A}$ \\
\hline $\begin{array}{l}\text { Energy } \\
\text { Conservation } \\
\text { Center, Japan }\end{array}$ & $\begin{array}{l}2010 \\
\text { (Oct) }\end{array}$ & 383 & 274 & 42 & 67 & available $^{80}$ & available & available \\
\hline
\end{tabular}

Table B-1. Available On-mode Power Data

75 Source: ENERGY STAR Qualified Televisions Specification revision Update, Jan 3, 2007

76 http://www.washingtonpost.com/wp-dyn/content/article/2010/02/21/AR2010022103688.html

77 http://www.sustainablebusiness.com/index.cfm/go/news.display/id/22024

78 http://www.eccj.or.jp/cgi-bin/real-catalog/index.php

79 http://www.eccj.or.jp/law06/machine/tv 220218.pdf

80 It is calculated from the below formula of annual energy consumption. .

$$
E_{\text {annual }}(k W h / y r)=\left\{P_{\text {on mode }} \times\left(\frac{4.5 h r s}{d a y}\right)+P_{\text {standby }} \times\left(\frac{19.5 h r s}{d a y}\right)\right\} \times 365
$$

http://www.eccj.or.jp/real-catalog/closet/pdf/Stv liquid.pdf 


\section{B] Average On-mode Power for Regions}

To determine the average on-mode power, we calculated the EEI of each TV model in the list, the average EEI of the size category, and the weighted regional/global market share. Table B-2 shows the volumeweighted EEI for LED backlit TVs.

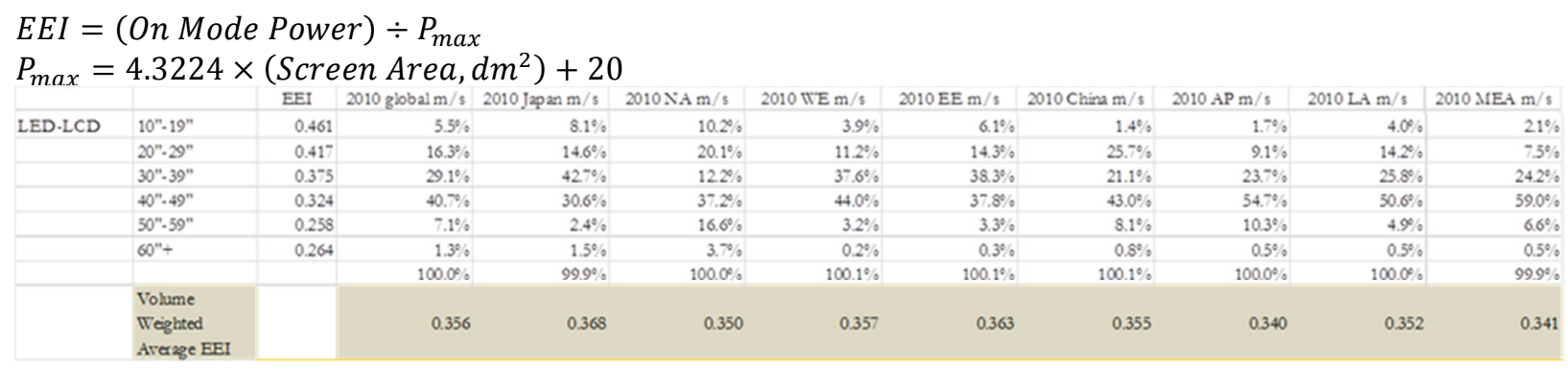

Source: ENERGY STAR 2010, DisplaySearch 2011a

Table B-2. Volume-weighted EEI for LED backlit TVs by Regions

Based on the average EEI, average screen area, and market share of each screen technology, the average onmode power for each region or country can be calculated using the formulas below:

Average On Mode Power for each region

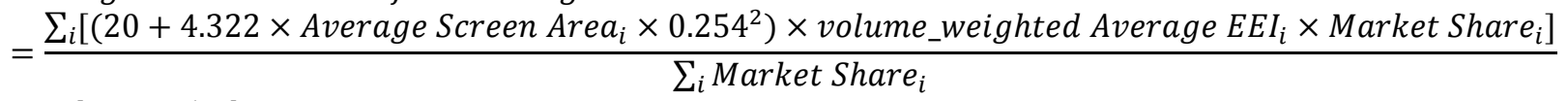

1 inch $=0.254 \mathrm{dm}$

, where $i$ represents each screen technology, e.g., CCFL-LCD, LED-LCD, PDP, and CRT.

Because we used screen technology and screen size market share data for the regional markets defined by DisplaySearch and referred only to the ENERGY STAR data, the average on-mode power values for all countries in the same region are identical. However, energy consumption and savings potentials are different for each country because of different viewing hours and country-specific TV shipments (or sales). Table B-3 shows the average on-mode power of each screen technology for each country in 2010. In the BUENAS modeling, OLED is assumed as a super-efficiency model in the LCD sector.

\begin{tabular}{l|l|r|r|r}
\hline Country/Region & \multicolumn{1}{|c|}{ Regional Market } & \multicolumn{1}{|c|}{ LCD } & \multicolumn{1}{c}{ PDP } & \multicolumn{1}{c}{ CRT } \\
\hline Australia & Asia Pacific & $68.0 \mathrm{~W}$ & $120.2 \mathrm{~W}$ & $52.7 \mathrm{~W}$ \\
\hline Canada & North America & $69.3 \mathrm{~W}$ & $116.0 \mathrm{~W}$ & - \\
\hline China & China & $73.1 \mathrm{~W}$ & $117.1 \mathrm{~W}$ & $60.1 \mathrm{~W}$ \\
\hline Western Europe & Western Europe & $63.2 \mathrm{~W}$ & $117.1 \mathrm{~W}$ & - \\
\hline Eastern Europe & Eastern Europe & $63.5 \mathrm{~W}$ & $113.4 \mathrm{~W}$ & $54.8 \mathrm{~W}$ \\
\hline India & Asia Pacific & $68.0 \mathrm{~W}$ & $120.2 \mathrm{~W}$ & $52.7 \mathrm{~W}$ \\
\hline Japan & Japan & $60.4 \mathrm{~W}$ & $111.1 \mathrm{~W}$ & - \\
\hline Korea & Asia Pacific & $68.0 \mathrm{~W}$ & $120.2 \mathrm{~W}$ & $52.7 \mathrm{~W}$ \\
\hline Mexico & Latin America & $70.2 \mathrm{~W}$ & $115.7 \mathrm{~W}$ & $52.2 \mathrm{~W}$ \\
\hline Russia & Eastern Europe & $63.5 \mathrm{~W}$ & $113.4 \mathrm{~W}$ & $54.8 \mathrm{~W}$ \\
\hline South Africa & Middle East \& Africa & $71.3 \mathrm{~W}$ & $114.3 \mathrm{~W}$ & $54.3 \mathrm{~W}$ \\
\hline US & North America & $69.3 \mathrm{~W}$ & $116.0 \mathrm{~W}$ & - \\
\hline
\end{tabular}

Table B-3. Average On-mode Power of Screen Technologies for Each Country in 2010

\section{C] Technology Options for Efficiency Improvement}

Technology options discussed in this report are based on manufacturer interviews, expert interviews, 
literature review, and recent international conferences/exhibitions, including the 2010 International Meeting on Information Display (IMID) in Korea, 2010 Flat Panel Display (FPD) International Conference in Japan, and 2011 Consumer Electronics Show in the U.S. Key interviewees have 7-15 years of experiences with the industry. Their collective expertise spans the TV market, LCD/PDP/OLED technologies, R\&D planning, and TV testing.

\section{Base Case / Efficiency Case Assumptions}

Base on the discussion in Chapter 4, this analysis compares future TV energy consumption for two scenarios: a base case and an efficiency case. The base case assumes options that are expected to be implemented regardless of any further policy action.

\begin{tabular}{|c|c|c|}
\hline & Base Case & Efficiency Case \\
\hline$P_{\text {display_LCD }}$ & $\begin{array}{l}\text { - Increase in LED efficacy } \\
\text { - Increase in panel transmittance } \\
\text { - Improvement in optical films } \\
\end{array}$ & $\begin{array}{ll}\cdot & \text { DBEF } \\
\cdot & \text { Dimming options } \\
\cdot & \text { LGP combined with prism } \\
\end{array}$ \\
\hline $\mathrm{P}_{\text {display_PDP }}$ & $\begin{array}{l}\text { - Improved cell structure } \\
\text { - High-density phosphors } \\
\text { - New electron emission layer } \\
\text { - Different discharge gas composition } \\
\text { - Low-voltage driving scheme }\end{array}$ & $\mathrm{N} / \mathrm{A}$ \\
\hline $\mathrm{P}_{\text {display_OLED }}$ & $\begin{array}{l}\text { - Improved light-emitting materials } \\
\text { - Improved TFT mobility }\end{array}$ & $\mathrm{N} / \mathrm{A}$ \\
\hline$P_{\text {basic }}$ & \multicolumn{2}{|c|}{ - $P_{\text {basic }}$ is assumed to be improved by $10-15 \%$ in 2014 from $20 \mathrm{~W}$ in 2010 . } \\
\hline
\end{tabular}

\section{Table B-4. Efficiency Scenarios applied to BUENAS model}

The optical film stack in LCD BLUs is assumed as follows: film stack without DBEF (base case) and film stack with DBEF (Efficiency Case). Because dimming options are currently available only in mid- and highend models, the base case does not include the effect of dimming on on-mode power. Because LGP combined with prism still faces technical barriers to implementation, the option is assumed to be available from 2013. As discussed in Chapter 4, it is difficult to quantify the individual effect of technology options for PDP TVs and OLED TVs. The improvement potentials for the two technologies are based on manufacturers' roadmaps and interviews. These are more illustrative than is the case for the LCD TV scenario.

\section{D] Average Screen Area}

Assumption: Average screen area for TVs in each country is the same as that of the corresponding region.

\begin{tabular}{l|c|c|c}
\hline & CCFL LCD TV & LED-LCD TVs & PDP TVs \\
\hline Japan & 30.2 & 33.6 & 44.8 \\
\hline NA & 33.3 & 39.2 & 48.2 \\
\hline WE & 31.1 & 35.9 & 45.6 \\
\hline EE & 31.2 & 35.4 & 44.8 \\
\hline China & 34.0 & 37.3 & 45.7 \\
\hline AP & 32.2 & 38.9 & 46.1 \\
\hline LA & 33.0 & 38.2 & 45.2 \\
\hline ME\&A & 33.1 & 38.9 & 45.1 \\
\hline
\end{tabular}

Source: DisplaySearch 2011a

\section{Table B-5. Average Screen Size (inches) for each Region in 2010 by Technology}

\section{E] Usage (viewing time per TV)}

Average viewing time and number of TVs per household are major factors affecting total energy 
consumption. Although further research is necessary to fully analyze these trends, we tried to identify recent country-specific data for average TV viewing time. Before discussing assumptions for our analysis, we review the following issues:

a. Survey Terminology: use of different terminology, e.g., on-time, viewing time (per individual, per household, per unit, and use time), may produce different results in surveys. Many surveys provide different results in terms of "the average TV viewing time." Some surveys might ask "How long does the respondent watch television per day?" and some others might ask "How long is the TV watched or video games played by all members of the household per day?" Our analysis considers both average daily viewing (on-mode) time per unit and regional TV shipments or sales.

b. TVs per household: the number of TVs per household in some countries is greater than one, so the average viewing time of secondary TVs in households for those countries needs to be considered because it is expected to be different from that of primary TVs. However, data are not available for country-specific secondary TVs and average viewing time. Therefore, for total viewing time for all TVs in a house hold, we multiplied on-mode power by the average number of hours per year that a TV is used in each country.

c. Growing Functionality: with growing functionality such as internet connectivity and 3D display capability, average viewing time may increase in the future; however the magnitude of such effects is still uncertain, needs further research, and is out of the scope of this report.

According to the OECD Communication Outlook (OECD 2009), the average "household TV viewing time" for many countries had been almost constant, under 4 hours, during the 10 years from 1997 to 2007 except in the U.S. The average American household's daily viewing hours were 7.7 in 2001 and 8.2 in 2007. The difference could be related to the average number of TV sets per household. ${ }^{81}$ That is, one possible explanation is that the OECD survey did not take into account multiple TVs operating in a household. In fact, the ENERGY STAR TV Specification Revision Update (ENERGY STAR 2007) uses a result from Nielsen Media Research (NMR) for the September 2004-September 2005 viewing season, which concluded that the average U.S. household was tuned into TV an average of 8 hours and 11 minutes per day. However, the EuP Preparatory Study (Fraunhofer 2007b) concluded that the figure does not necessarily indicate the average daily on-mode time of a single TV; it could mean that multiple TVs are operating at different times or in parallel in a household.

Thus, it is necessary to compare the OECD data with other studies or surveys. According to the International Energy Agency (IEA) 4E Mapping and Benchmarking report (IEA 2010), viewing hours vary by country. Viewing hour figures for the UK (4.8h), Australia (7.3h), and the Republic of Korea (6.9h) were based on government assumptions. However, the document mentioned that no underlying evidence was available for the data, so assessment of robustness was not possible. The EuP Preparatory Study (Fraunhofer 2007b), which includes many studies that provide data on TV watching time, mentioned that "the existing results from studies on TV use patterns indicate a typical on-mode time duration per TV per day in European households of 2.5 to 5 hours. The range reflects the increasing of a second TV in households." The study consequently assumed that average viewing time, i.e., daily on-time duration of the primary TV in a European household, is 4 hours per day. The 4E Document also assumed an average of 4 hours per day for EU 15 countries, according to the EuP Preparatory Study. Table E-4 shows TV viewing time estimates from various studies, which are included in the EuP Preparatory Study.

81 The average number of TV sets per household in 2009 for the U.S. was estimated at 2.86 (IEA 2009). 


\begin{tabular}{|c|c|c|c|c|c|c|}
\hline Study/Source & Year & Countries & $\begin{array}{l}\text { On-Mode } \\
(\mathrm{h} / \mathrm{d})\end{array}$ & $\begin{array}{l}\text { Standby } \\
(\mathrm{h} / \mathrm{d})\end{array}$ & $\begin{array}{l}\text { Off-Mode } \\
\text { (h/d) }\end{array}$ & $\begin{array}{l}\text { Main off } \\
(\mathrm{h} / \mathrm{d})\end{array}$ \\
\hline DOE & 1998 & US & 4 & 20 & - & - \\
\hline Jupiter Research & 2006 & $\begin{array}{l}\text { UK, France, } \\
\text { Germany, } \\
\text { Italy, Spain }\end{array}$ & $1.7^{*}$ & - & - & - \\
\hline Buhl data & 2006 & Germany & $\begin{array}{l}2.2 \text { digital* } \\
3.8 \text { all }^{*}\end{array}$ & - & - & - \\
\hline $\begin{array}{l}\text { nVision/Future } \\
\text { Foundation }\end{array}$ & 2006 & EU & $2 *$ & - & - & - \\
\hline $\mathrm{AGF} / \mathrm{GfK}$ & 2006 & Germany & $3.5^{*}$ & & & \\
\hline ISI, CEPE & 2003 & Germany & $\begin{array}{l}2005: 4.7 \\
2010: 5\end{array}$ & $\begin{array}{l}12.1 \\
16.8\end{array}$ & $\begin{array}{l}3.6 \\
1.1\end{array}$ & $\begin{array}{l}3.6 \\
1.1\end{array}$ \\
\hline forsa, RWI & 2003 & Germany & 4.8 & - & - & - \\
\hline $\begin{array}{l}\text { Meyer, } \\
\text { Schaltegger }\end{array}$ & 1999 & Switzerland & 2.3 & 9.7 & 12 & - \\
\hline EICTA CoC & 2003 & EU & 4 & 16 & (12 if APO) & - \\
\hline GEEA & 2001 & $\begin{array}{l}\text { GEEA } \\
\text { framework }\end{array}$ & 4 & $0-20$ & $0-16$ & - \\
\hline $\begin{array}{l}\text { European } \\
\text { Communities }\end{array}$ & 2003 & EU & $3.5^{*}$ & - & - & - \\
\hline $\begin{array}{l}\text { Nielsen Media } \\
\text { Research }\end{array}$ & 2006 & USA & $8^{*}$ & - & - & - \\
\hline
\end{tabular}

* Average watching time, not over all operating time

Source: Fraunhofer 2007b

Table B-6. Estimates of "On," "Standby," and "Off" times for TVs

In addition to the above studies, we used recent survey results. Table E-5 summarizes various data sets we have. 


\begin{tabular}{|c|c|c|c|c|c|}
\hline Study/Survey & $\begin{array}{c}\text { OECD } \\
\text { (average for given } \\
\text { years) }\end{array}$ & $\begin{array}{c}4 \mathrm{E} \\
\text { Benchmarking }^{82}\end{array}$ & $\begin{array}{l}\text { IP-Network } \\
\text { (average for }^{83} \\
\text { given years) }\end{array}$ & TGI Surveys ${ }^{84}$ & $\begin{array}{c}\text { LBNL } \\
\text { (assumptions) }\end{array}$ \\
\hline $\begin{array}{l}\text { Title of } \\
\text { Study/Survey }\end{array}$ & $\begin{array}{l}\text { viewing time } \\
\text { per household }\end{array}$ & $\begin{array}{c}\text { viewing time } \\
\text { per TV }\end{array}$ & $\begin{array}{l}\text { viewing time } \\
\text { per individual }{ }^{85}\end{array}$ & $\begin{array}{l}\text { viewing time } \\
\text { per individual }\end{array}$ & $\begin{array}{l}\text { viewing time } \\
\text { per TV }\end{array}$ \\
\hline Years covered & 1997 to 2007 & 2007 to 2009 & 2007 to 2009 & 2009 & 2010 \\
\hline Australia & 3.21 & 7.3 & 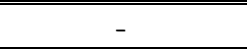 & 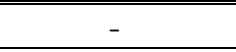 & 6.5 \\
\hline Canada & $3.80^{86}$ & - & - & 3.2 & 4.5 \\
\hline China & - & - & - & 3.49 & 4 \\
\hline France & $3.36^{87}$ & 5 & 3.62 & 2.86 & 5 \\
\hline Germany & 3.34 & - & 3.72 & 3 & 5 \\
\hline India & - & - & - & 2.5 & 3.5 \\
\hline Japan & 3.70 & - & 3.69 & 3.77 & 4.5 \\
\hline Korea & $3.11^{88}$ & 6.9 & - & 2.34 & 6 \\
\hline Mexico & - & - & - & 3.83 & 4 \\
\hline Russia & - & - & 4.03 & - & 4 \\
\hline South Africa & - & - & - & - & 4 \\
\hline Sweden & 2.5489 & - & 2.85 & - & 3.5 \\
\hline $\mathrm{UK}$ & 3.3990 & 4.8 & 3.97 & - & 5 \\
\hline US & 7.75 & 5 & 3.96 & - & 5 \\
\hline $\mathrm{EU}$ & - & 4 & $3.79^{91}$ & - & 4 \\
\hline
\end{tabular}

Unit: hours per day

\section{Table B-7. Average TV Viewing Time from Various Sources}

Although some surveys of average TV viewing time cover many countries, most surveys focus on "average viewing time per individual or household" rather than "average viewing time per TV," which is a more useful metric when discussing the impact of TV efficiency improvements on energy consumption. The OECD report provided historical data for many countries, but these are average TV viewing hours per household. The 4E Document provided average viewing time per TV does not assess the robustness of the data. In addition, it is generally expected that average viewing time per individual becomes less than average viewing time per TV as the number of members in a household is increasing. It is difficult to determine the comparative robustness of the various data sets due to the multiple variables. Because of lack of data as well as differences among data sets, we assume average viewing hours per unit for countries as follows: between 3.5 and 5 hours for all countries except for Australia and Korea where we assume 6.5 and 6 hours, respectively, which are close to the figures in the 4E Document.

\section{- Australia: 6.5 hours}

There is a significant difference between the OECD Report (viewing time per household) and the 4E Benchmarking Document (viewing time per TV), which was based on government assumptions.

\footnotetext{
82 According to the 4E Benchmarking Document, the data for Australia, Korea, and United Kingdom are based on government assumptions, and the data for France, EU, and the U.S. are assumed from other studies.

83 IP-Network 2010

84 TGI 2009

85 Average viewing time per week

86 From the years 2005 to 2007

87 From the years 2000 to 2007

88 The years 1997, 2004, 2006, and 2007

89 From the years 2005 to 2007

90 From the years 2002 to 2007

91 Average of EU 26 without Malta
} 
A TV expert ${ }^{92}$ from Australia suggested that this figure should be more than 6 hours based on other surveys. Therefore, this analysis assumes an average 6.5 hours per TV for Australia.

- Canada: 4.5 hours

According to BBM Canada, adults over 18 years old watched 29.1 average weekly hours, i.e., 4.16 daily hours, of television as of July $2010 .{ }^{93}$ This figure looks not very different from the OECD report's estimate of 3.8 hours of average viewing time per household. This analysis assumes an average 4.5 hours per TV for Canada.

- China: 4 hours

One study reported the average TV viewing time per individual per day in China was 179 minutes, i.e., 3 hours, in 2003 (Wang 2008). A recent survey from TGI provided an average of 3.5 hours per individual as seen Table B-7. This analysis assumes an average of 4 hours per TV for China.

- France: 5 hours

The OECD report and other surveys indicate about 3-4 hours as the average viewing time per household or individual in France, but the 4E Document assumes 5 hours per TV. This analysis also assumes an average 5 hours per TV for France.

- Germany: 5 hours

We apply to Germany the same assumptions as used for France.

- India: $\mathbf{3 . 5}$ hours

The TGI survey indicates an average 2.5 hours per individual in India, and there are no other good sources of data for comparison. We assumed that the average viewing time per TV would be higher than that, even though it might be less than 4 hours, a generally accepted global average. This analysis assumes an average 3.5 hours per TV for India.

- Japan: 4.5 hours

Although all sources in Table B-7 indicated about 3.7 hours as average viewing time per individual in Japan, ECCJ assumed 4.5 hours per TV for energy consumption estimates for recent TV models. This analysis assumes 4.5 hours per TV for Japan.

- Korea: 6 hours

There is a significant difference in data on Korean TV viewing in the OECD Report (viewing time per household) and the 4E Benchmarking Document (viewing time per TV) which was based on government assumptions. In the 4E report, Korean TVs were in on mode for average of 6.9 hours per day, 351 days per year. According to AGB Nielsen Media Research, the average TV viewing time per household in Korea from the first quarter of 2009 to the first quarter of 2010 was about 9 hours. ${ }^{94}$ The average number of TVs in Korean households was about 1.5 between 2007 and 2009 (IEA 2010a). It is possible that respondents to the AGB Nielsen survey included hours they spend watching TV programs via other devices such as PCs and mobile phones. This figure might be higher than an average viewing time per TV. This analysis assumes an average 6 hours per TV for Korea.

- Mexico: 4 hours

The TGI survey gives an average of 3.8 hours TV viewing time per individual in Mexico. There are no other good data sources for comparison. This analysis assumes an average 4 hours per TV for Mexico.

- Russia: 4 hours

The IP-Network report gives an average 4 hours of TV viewing per individual in Russia. There are no other good data sources for comparison. This analysis assumes an average 4 hours per TV for Russia.

\section{- South Africa: 4 hours}

\footnotetext{
92 Comment from Keith Jones of Digital Cenergy in the first round review of this report.

93 http://www.tvb.ca/pages/tv-pvr.htm

94 http://www.newswire.co.kr/newsRead.php?no $=466533 \& \operatorname{lmv}=\mathrm{A} 03$
} 
This analysis does not have good sources to evaluate average TV viewing time in South Africa. We assume an average of 4 hours per TV, a generally accepted average viewing time per TV, for South Africa.

\section{- Sweden: 3.5 hours}

Both the OECD report and the IP-Network report give figures of less than 3 hours per household or individual for TV viewing in Sweden, and this analysis does not have other good data sources for comparison. Anecdotal evidence is that average TV viewing time in Sweden is less than in other countries. This analysis assumes an average 3.5 hours per TV for Sweden.

- United Kingdom: 5 hours

The OECD report and other surveys give 3 to 4 hours for the average viewing time per household or individual in the United Kingdom, and the 4E Document gives 4.8 hours per TV based on government assumptions. This analysis assumes an average 5 hours per TV for the United Kingdom.

\section{- United States: 5 hours}

There is a significant difference between the OECD Report (viewing time per household) and other studies figures for the U.S. As discussed above, one possible explanation for the figure in the OECD report is that the OECD survey did not take into account multiple TVs running in a household. According to Nielsen's Three Screen Report (Nielsen 2009), the average American watches approximately 153 hours every month, i.e., 5.1 hours per day, of TV at home. ENERGY STAR uses an average of 5 hours to estimate energy consumption by TVs. This analysis also assumes an average 5 hours per TV for the U.S.

\section{- EU: 4 hours}

For EU $15^{95}$, the $4 \mathrm{E}$ Document assumes an average of 4 hours of daily viewing time per TV. This analysis assumes the same.

Although average viewing time per TV is a major factor affecting total energy consumption, country-specific data for average TV viewing time vary with studies. Further research is necessary to fully analyze these trends. Thus, energy consumption data for each country in this report need to be carefully compared to each other, separately considering average viewing time from average on-mode power for each country.

\footnotetext{
95 Austria, Belgium, Denmark, Finland, France, Germany, Greece, Ireland, Italy, Luxembourg, Netherlands, Portugal, Spain, Sweden,
} and United Kingdom 


\section{Appendix C. Sensitivity Analysis of Energy-Efficiency Improvement Options}

In this appendix, we examine relationships between efficiency improvement options and total TV energy consumption. In general, a TV's annual unit energy consumption (UEC) is expressed as a function of onmode power, on-mode viewing time, standby power, and usage in standby mode.

Unit Energy Consumption $(U E C, k W h /$ year $)=f\left(P_{\text {on-mode }}, t_{\text {on mode }}, P_{\text {standby }}, t_{\text {standby mode }}\right)$

For recent FPD TVs, the efficiency is affected by various technical options such as display-specific efficiency, brightness control, basic power, and PSU efficiency. Although daily viewing time per TV is an important factor in a TV's total energy consumption, it is hard to control with policy instruments, and robust data are not available. Although a TV's passive standby power consumption (typically less than $1 \mathrm{~W}$ ) is much less than on-mode power, it is likely that network standby mode could become a significant source of energy consumption. Therefore, this sensitivity analysis focuses on network standby power and key technical factors related to on-mode power.

\section{LCD T'Vs}

For LCD TVs, TV on-mode power $\left(P_{\text {on-mode }, L C D}\right)$ can be generally expressed as a function of screen area, screen efficiency (e.g., watts per screen area), and basic power.

$P_{\text {on-mode }, L C D}=g($ screen size, $B L U$ efficiency, panel transmittance, brightness control, basic power, $P S U$ efficiency)

For LCD TVs, display efficiency is affected by various technical options such as backlight source efficacy, optical film stack efficiency, panel transmittance, brightness control, and basic power. Let's assume that a 32" LED-LCD TV consumes $60 \mathrm{~W}$ in on-mode. Other assumptions are as follows:

- The basic power (included in the on-mode power) is $20 \mathrm{~W}$.

- Default luminance ${ }^{96}$ of the TV is $300 \mathrm{~cd} / \mathrm{m}^{2}$.

- LCD panel transmittance is 5\%.

- The average efficacy of LEDs in the BLU is $60 \mathrm{~lm} / \mathrm{W}$.

- $\quad$ PSU efficiency is $100 \% .{ }^{97}$

- No ABC and dimming options are assumed.

- Average daily on-mode viewing time is 5 hours.

- Average network standby power is $4 \mathrm{~W}$.

- Average daily usage on network standby mode is 19 hours.

In this base case, the annual energy consumption by the TV is $137 \mathrm{kWh}$ per year. If each variable - screen area $\left(\mathrm{X}_{1}\right)$, LED efficacy $\left(\mathrm{X}_{2}\right)$, panel transmittance $\left(\mathrm{X}_{3}\right)$, default brightness $\left(\mathrm{X}_{4}\right)$, basic power $\left(\mathrm{X}_{5}\right)$, and network standby power $\left(\mathrm{X}_{6}\right)$ - independently increases or decreases by $50 \%$ from the above baseline with other variables constant, the annual energy consumption by the TV varies as in Figure C-1 and C-2.

\footnotetext{
96 LCD TV panels larger than 30 inches are manufactured with a target luminance of 400 to $500 \mathrm{~cd} / \mathrm{m}^{2}$. In general, this luminance level corresponds to retail mode or brightest selectable mode in a TV. We assumed that a TV's default luminance as shipped corresponds to the luminance at on-mode power test. The average luminance in default mode as shipped of a 32" LCD ENERGY STAR-qualified TV is about $270 \mathrm{~cd} / \mathrm{m}^{2}$, and the average luminance in the brightest selectable preset mode of those TVs is about $360 \mathrm{~cd} / \mathrm{m}^{2}$.

97 Although, in reality, the efficiency of the PSU is now between $85 \%$ and $95 \%$, we did not include this factor in this analysis.
} 


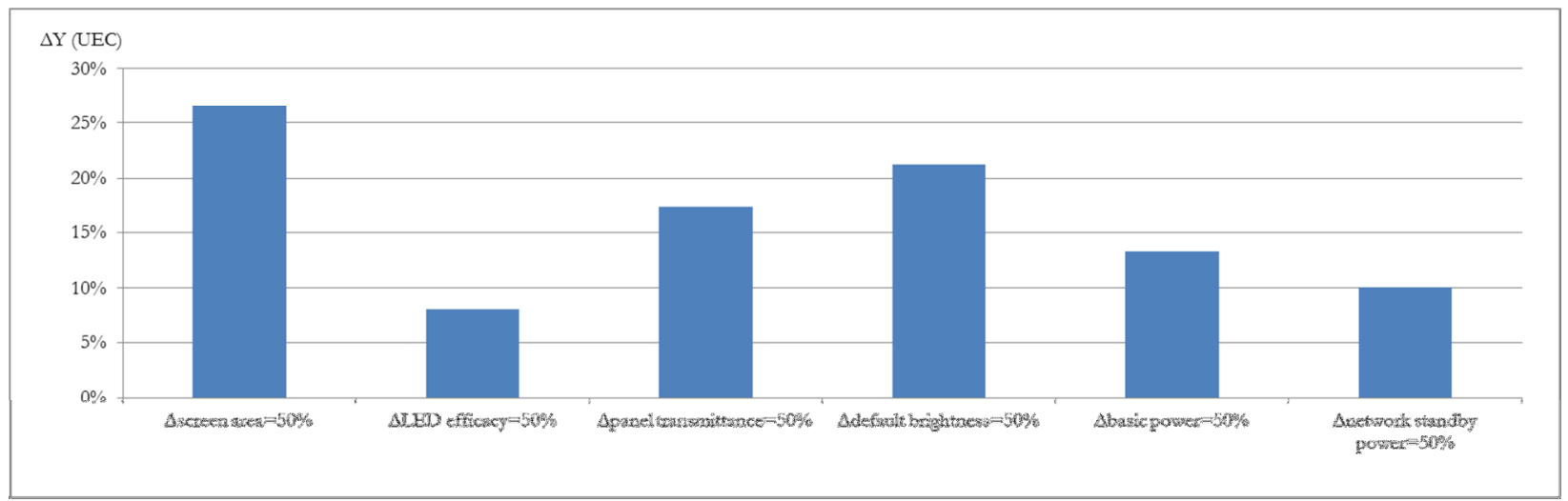

Figure C-1. UEC Sensitivity to Technical Factors (32" LED-LCD TV, $P_{\text {network standby }}=4 \mathrm{~W}$ )

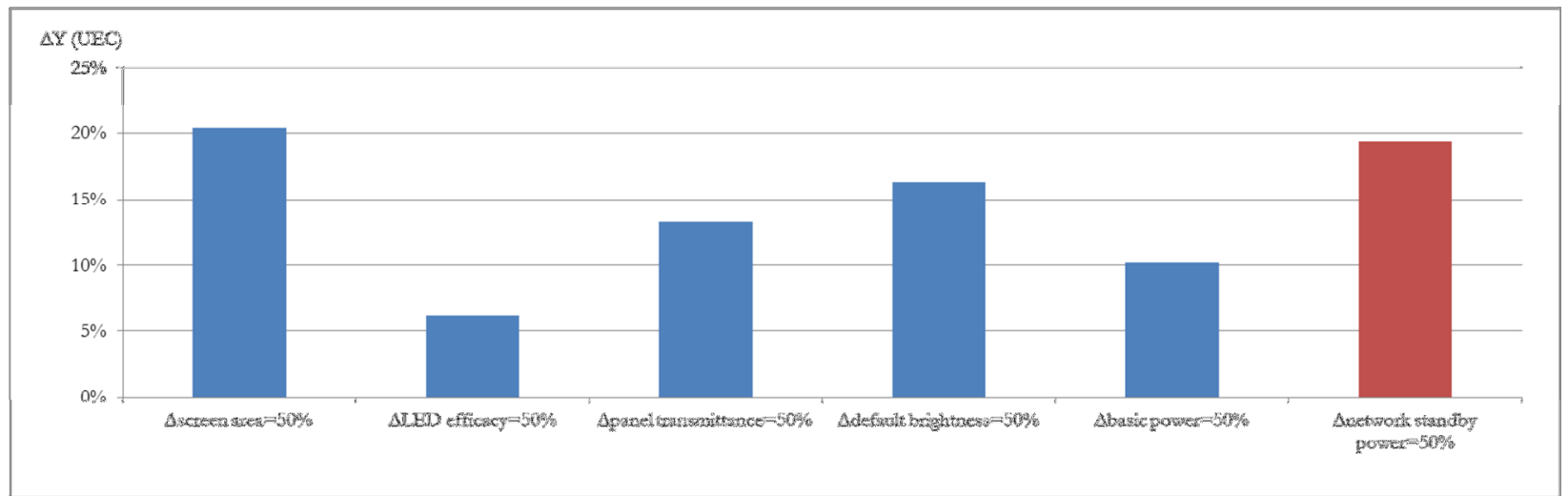

Figure C-2. UEC Sensitivity to Technical Factors (32" LED-LCD TV, $P_{\text {network standby }}=10 \mathrm{~W}$ )

A] Screen Area: $\Delta \mathrm{X}_{1}=50 \% \rightarrow \Delta \mathrm{Y}=20-27 \%$

The screen area of a 32" LCD TV is about 437 square inches. If the area increases by $50 \%$, the new area is about 656 square inches, which is equivalent to a 39 " screen size diagonal. We can assume that the screen area increase does not affect the basic power, so the expected on-mode power for this screen area increase is $80 \mathrm{~W}$. The average of ENERGY STAR-qualified TVs with ABC disabled is about $80 \mathrm{~W}$, within the range of $60 \mathrm{~W}$ and $110 \mathrm{~W}$. As seen in Figure D-1 and D-2, UEC is the most sensitive to screen area.

B] LED Efficacy: $\Delta \mathrm{X}_{2}=50 \% \rightarrow \Delta \mathrm{Y}=6-10 \%$

As discussed in Section 4.1.2., even though LED efficacy increases by $50 \%$, from $60 \mathrm{~lm} / \mathrm{W}$ to $90 \mathrm{~lm} / \mathrm{W}$, the effect of on both on-mode power and energy consumption is not likely to be significant because LED power should increase.

C] Panel Transmittance: $\Delta \mathrm{X}_{3}=50 \% \rightarrow \Delta \mathrm{Y}=13-17 \%$

Improvement in LCD panel transmittance allows manufacturers to reduce the number of LEDs from the BLU because the luminance that the backlight must achieve decreases. If panel transmittance is improved by $50 \%$, from $5 \%$ to $7.5 \%$, the total on-mode power is expected to decrease by about $20 \%$, and the UEC decreases by about 15\% from the base line. According to a manufacturer, if panel transmittance is improved from $6.5 \%$ to over $8 \%$ and some other technologies are applied, the power consumed by a 32HD LCD panel could decrease by more than $35 \%$, e.g., $32 \mathrm{~W}$ to $20 \mathrm{~W}$. Although panel transmittance increases up to $7 \%$ to $8 \%$ are expected from some manufacturers in a few years, this option requires R\&D investment rather than incremental costs. 


\section{D] Default Brightness: $\Delta X_{4}=50 \% \rightarrow \Delta Y=16-20 \%$}

Decreasing the luminance of a TV set can provide a relatively straightforward on-mode power reduction. Let's assume that the default luminance as shipped corresponds to the luminance at on-mode power test. If the luminance decreases by $50 \%$, from $300 \mathrm{~cd} / \mathrm{m}^{2}$ to $150 \mathrm{~cd} / \mathrm{m}^{2}$, its on-mode power is expected to decrease by about $25 \%$ and annual energy consumption by about $20 \%$. Brightness control functions for TVs are implemented as a form of $\mathrm{ABC}$ or a local dimming option. Manufacturers continue to consider the option of reducing default brightness to reduce both power and materials costs. It is important to develop test methods to appropriately evaluate the effect of brightness control functions in TVs.

\section{E] Basic Power: $\Delta \mathrm{X}_{5}=50 \% \rightarrow \Delta \mathrm{Y}=10-13 \%$}

For a 32" LED-LCD TV that consumes $60 \mathrm{~W}$ in on mode, basic power accounts for about $30 \%$. If basic power is improved by $50 \%$, from $20 \mathrm{~W}$ to $10 \mathrm{~W}$, the on-mode power is expected to decrease by about $15 \%$ and the annual energy consumption by about 13\%. Improvement in basic power mainly depends on development of integrated circuit technologies and a power reduction algorithm. It does not seem to be an appropriate cost-effective option.

\section{F] Network Standby Power: $\Delta \mathrm{X} 6=50 \% \rightarrow \Delta \mathrm{Y}=10-20 \%$}

If average network standby power is assumed to be $4 \mathrm{~W}$, changing network standby power has a relatively small effect on annual energy consumption. However, robust data for average network standby power are not available for this report, so it is necessary to consider higher average network standby power. In Section 6.4., we assumed network standby power of a connected TV was between $3 \mathrm{~W}$ and $30 \mathrm{~W}$. If we assume average network standby power of $10 \mathrm{~W}$, for illustrative purposes, the effect of standby power on UEC becomes significant because on-mode usage is generally less than standby usage (See Figure C-2). Although it is difficult to determine the average standby power of connected TVs including network standby mode, it is important to reduce the average network standby power of TVs by power management regimes that allow TVs to enter low-power mode while connected but not in use.

To summarize, it is necessary to develop or revise existing test procedures/regulations to appropriately evaluate brightness control functions in TVs and to prevent possible increases in energy consumption by network standby power. Although this report concludes that only optical films and dimming options are costeffective options, high-efficiency LEDs at low to medium power and LCD panels with high transmittance could be options for R\&D investment. However, it is important to note that LCD panel technologies are proprietary and closely related to manufacturing processes. Also, some of these options are interdependent to some extent. If all options are applied, the aggregate effect will be different from a simple summation of individual effects. 


\section{Appendix D. Cost-Efficiency Relationship}

In this report we analyzed three cost-effective options for LCD TVs. While the two cost-effective options, DBEF and dimming technology, can be applied regardless of screen size, some TV models at the higher end of the large screen sizes already employ these options. Although prism patterned LGP is discussed as a costeffective option, it still needs to overcome technical issues. In this appendix, we provide cost-efficiency relationships for DBEF and dimming options.

\section{D1. Cost-Efficiency Table}

Figure D-1 shows cost-efficiency relationships for DBEF and dimming option. As the effect of DBEF is assumed to improve screen efficiency by $20 \%$, the effect of DBEF on on-mode power varies with screen size. As the effect of dimming option (0D or 1D dimming) is assumed to improve screen efficiency by $15 \%$ at a minimum level, the effect of dimming on on-mode power varies with screen size. It is important to note that to quantify the effect of dimming, it is necessary to measure power consumption using various dimming methods with the IEC 62087 standard test video clip. However, these data are not available in this report.

\begin{tabular}{l|r|r|r}
\hline & & \multicolumn{2}{|c}{ Incremental Costs } \\
\hline Product Class & Screen Size & $\begin{array}{c}\text { DBEF } \\
\left(\Delta \text { Pon-mode: }^{9}-17 \% \downarrow\right)\end{array}$ & $\begin{array}{c}\text { Dimming } \\
(\Delta \text { P on-mode } 7-13 \% \downarrow)\end{array}$ \\
\hline \multirow{2}{*}{$\begin{array}{l}\text { LED backlit } \\
\text { LCD TV }\end{array}$} & 26 & $\$ 5.0(\Delta 9 \%)$ & $\$ 0.8-1.5(\Delta 7 \%)$ \\
\cline { 2 - 4 } & 32 & $\$ 7.6(\Delta 13 \%)$ & $\$ 3.2-6.0(\Delta 10 \%)$ \\
\cline { 2 - 4 } & 37 & $\$ 9.9(\Delta 14 \%)$ & $\$ 3.2-6.0(\Delta 11 \%)$ \\
\cline { 2 - 4 } & 40 & $\$ 12.7(\Delta 15 \%)$ & $\$ 3.2-6.0(\Delta 11 \%)$ \\
\cline { 2 - 4 } & 42 & $\$ 13.6(\Delta 16 \%)$ & $\$ 3.2-6.0(\Delta 12 \%)$ \\
\cline { 2 - 4 } & 46 & $\$ 16.3(\Delta 16 \%)$ & $\$ 3.2-6.0(\Delta 12 \%)$ \\
\cline { 2 - 4 } & 47 & $\$ 17.0(\Delta 16 \%)$ & $\$ 3.2-6.0(\Delta 12 \%)$ \\
\cline { 2 - 4 } & 52 & $\$ 23.1(\Delta 16 \%)$ & $\$ 4.8-9.0(\Delta 12 \%)$ \\
\hline
\end{tabular}

* The above information is based on 2010 data.

\section{Table D-1. Cost-Efficiency Table for LCD TVs}

\section{D2. Cost of Conserved Energy (CCE)}

Based on the cost-efficiency relationship and global TV shipment forecast data, we can analyze cost of conserved energy (CCE) for each product class which is divided by screen size. Since some TV models at the higher end of the large screen sizes already employ these options, it is hard to forecast the share of entry or mid-end TVs without those options in the market. The assumptions used in this calculation are as follows:

- $\quad 70 \%$ of global sales in 26"-39" LED backlit LCD TVs will not adopt these cost-effective options in the absence of energy efficiency policies. The same assumption applied to $40 \%$ of $40 "-49$ " LED backlit LCD TVs and $20 \%$ of 50"-55" LED backlit LCD TVs.

- An effective useful life of 10 years and a discount rate of $6 \%$ are assumed.

- Costs of DBEF and dimming drive ICs will decrease by 30\% from 2010 to 2012.

Figure D-1 and D-2 show costs of conserved energy for these cost-effective options in this scenario. Figure D-1 shows annualized incremental cost of conserved energy by product class for DBEF. Most products have a cost of conserved energy less than 8 cents per $\mathrm{kWh}$. Although we assumed small market penetration rates for large screen size over 50 inches, 32"-42" products account for more than $90 \%$ of cumulative savings in this scenario. 


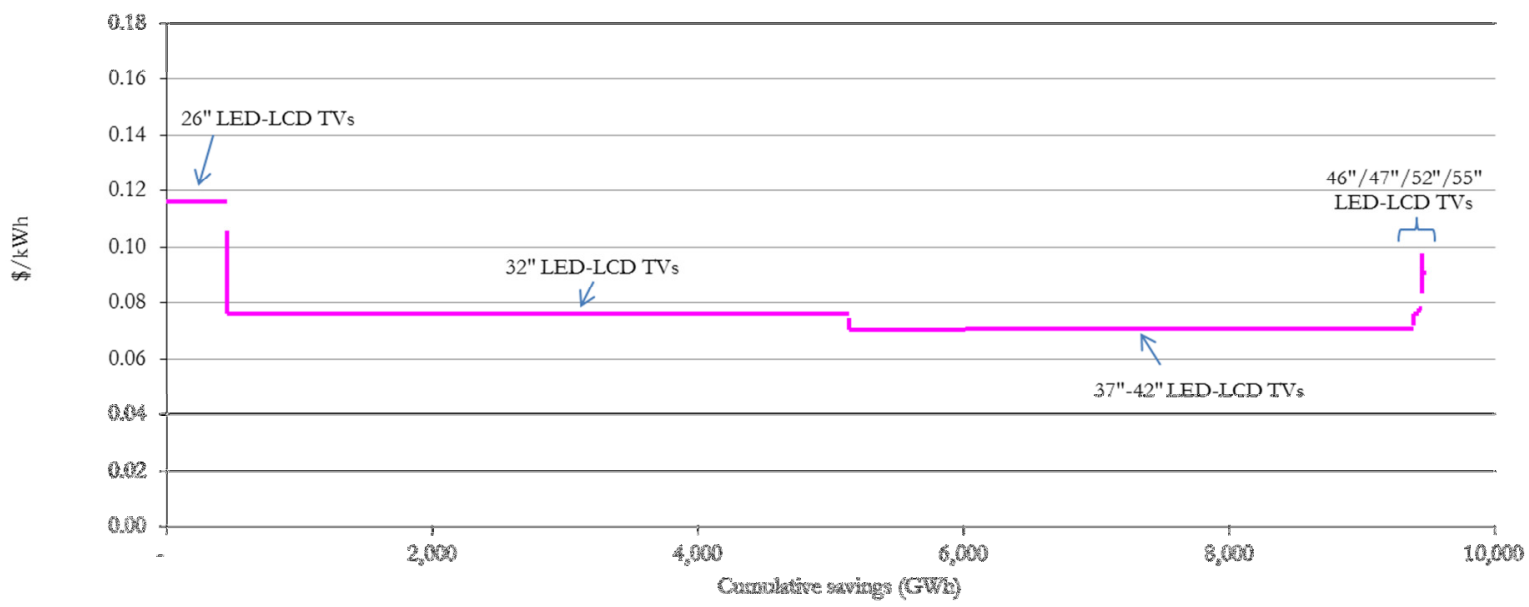

Figure D-1. Cost of Conserved Energy for DBEF (2012 LED-LCD TVs)

Figure D-2 shows annualized incremental cost of conserved energy by product class for dimming option. The difference between pink line and blue line shows the range of incremental cost required for dimming option. Most products have a cost of conserved energy less than or equal to 8 cents per $\mathrm{kWh}$ at a maximum level. While DBEF cost increases depending on screen area, number of dimming drive ICs is less sensitive to screen size. Although we assumed small market penetration rates for large screen size over 50 inches, 32"-42" products account for more than $90 \%$ of cumulative savings in this scenario.

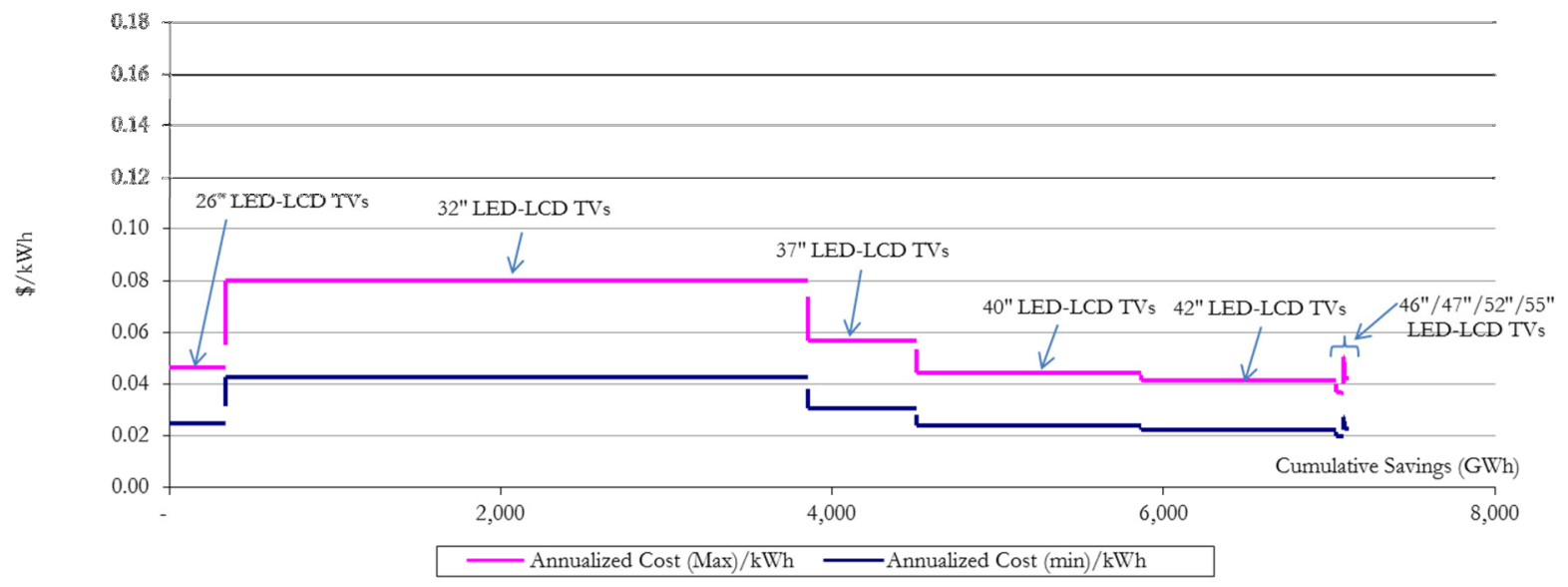

Figure D-2. Cost of Conserved Energy for Dimming Option (2012 LED-LCD TVs) 


\section{Appendix E. Brightness and Luminance}

Although we tried to use technical terms carefully and consistently in this report, readers might be confused in some instances. Particularly, this may be the case for the terms "brightness" and "luminance", which many people, even manufacturers or researchers, use interchangeably and inconsistently. Most people are used to the term brightness as a picture setting on their display, and therefore the term brightness may be selfexplanatory. So, it would be useful to briefly discuss the technical distinction between luminance (measured at the screen) and brightness (perceived by the viewer).

Luminance is a photometric measure of the luminous intensity of light per unit area travelling in a given direction. It is a measured value from a photo-detector described in the form of candela per square meters $\left(\mathrm{cd} / \mathrm{m}^{2}\right)$. Luminance is a measurable quantity which corresponds to brightness, but the relationship between them is non-linear and complex. (ENERGY STAR 2011b, Halsted 1993, Wikipedia 2011b) Table E-1 shows conceptual definitions and general mathematical forms of technical terms relevant to luminance.

\begin{tabular}{|c|c|c|}
\hline Term & Definition & unit \\
\hline Luminance & $\begin{array}{l}\text { Luminous intensity of light per } \\
\text { unit area (measured brightness } \\
\text { of a surface) }\end{array}$ & $L=\frac{I(\text { candela }=c d)}{m^{2}}=\frac{\operatorname{lumen}(\mathrm{lm}) / \text { solid angle }(\text { sr })}{m^{2}}=$ nit \\
\hline Luminous intensity & $\begin{array}{l}\text { Luminous flux (quantity of light } \\
\text { emitted per unit time in a } \\
\text { particular direction) per unit } \\
\text { solid angle }\end{array}$ & $I=\frac{F(\text { lumen })}{\omega(s r)}$ \\
\hline Luminous flux & $\begin{array}{l}\text { Perceived quantity of light per } \\
\text { unit time (visible power of light) }\end{array}$ & $F($ lumen $)=\frac{d Q}{d t}$ \\
\hline Solid angle $(\omega, \Omega)$ & $\begin{array}{l}\text { Two-dimensional angle in three- } \\
\text { dimensional space that an object } \\
\text { subtends at a point }\end{array}$ & $\omega=\frac{S}{r^{2}}$ \\
\hline
\end{tabular}

Source: Halsted 1993, Wikipedia 2011b

\section{Table E-1. Concepts Relevant to Luminance}

Brightness is a subjective attribute of visual perception elicited by the luminance of an object, in which viewers determine a degree between "very dim" and "very bright". Brightness is what is perceived by the viewer and is affected by the magnitude of the light and environmental factors such as ambient light. Although the luminance photometer and the human eye both receive light from specific directions, the perceived results may be different because the photometer detects the light through a single detector while the human eye does the process through a large number of organic sensors, as well as due to dilation or narrowing of the iris. As brightness is what is perceived, not measured, the human eye's response is nonlinear to change in luminance. The sensitivity of the eye decreases as the magnitude of the light increases. (Chinnock 2011, Halsted 1993) For this reason, ABCs in TVs do not change the brightness linearly as the room lighting changes linearly. Usually, when the room illuminance reaches a certain level, the brightness decreases to a level which viewers feel adequate for the room conditions. Figure E-1 shows the relationship between luminance and eye response. 


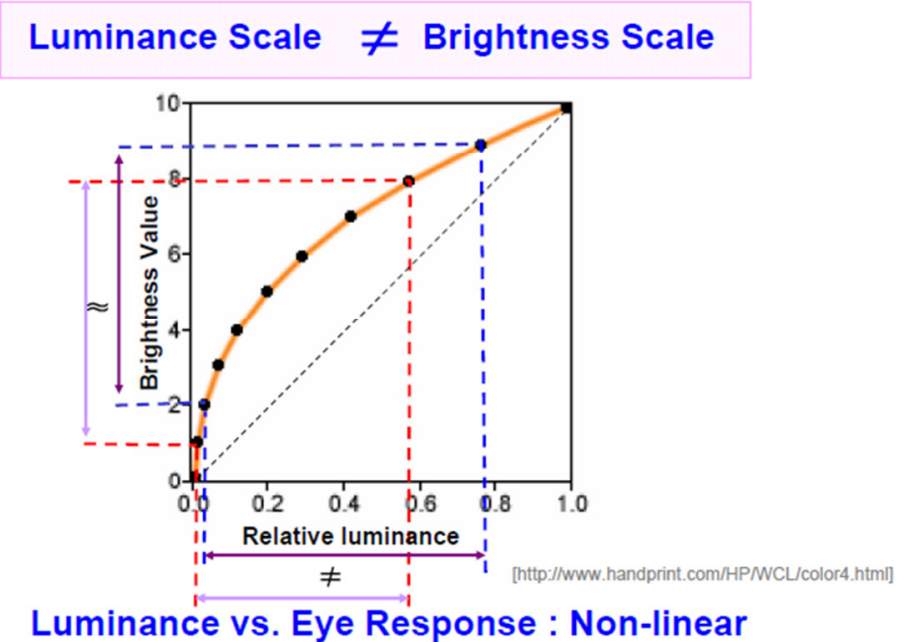

Source: Lee 2008

Figure E-1. Luminance vs. Eye Response

When we discussed 3D TV brightness in the report, it indicated that technical light loss occurred in 3D mode of a 3D-capable TV may result that viewers may experience relatively low brightness level in 3D mode compared to 2D mode although screen luminance is the same or above by automatic adjustment. Although 3D perception renders the image subjectively brighter than it technically is due to light loss, current 3D TVs need to be improved further to allow an effective brightness level that is comfortable for the viewer. 